\title{
Photochemical O-H Functionalization of Aryldiazoacetates with Phenols via Proton Transfer
}

Claire Empel, ${ }^{[a, b]}$ Sripati Jana, ${ }^{[a]}$ Chao Pei, ${ }^{[a]}$ and Thanh Vinh Nguyen, ${ }^{*[b]}$ Rene M. Koenigs*[a,b]

[a] Institute of Organic Chemistry, RWTH Aachen University, Landoltweg 1, D-52074 Aachen, Germany

E-mail: rene.koenigs@rwth-aachen.de

[b] School of Chemistry, University of New South Wales, Sydney 2052, Australia

E-mail: t.v.nguyen@unsw.edu.au

\section{Table of Content}

General Information $\quad$ S1

Important Safety Note $\quad$ S1

$\begin{array}{ll}\text { Experimental Procedures } & \text { S2 }\end{array}$

Data on Chemical Shift Perturbations, Resonant Frequencies, $\mathrm{p} K_{\mathrm{A}}$ Values,

Energy of Intramolecular Hydrogen Bond Strength and Yield $\quad$ S3

Chemical Shift Perturbation vs. Experimental $\mathrm{p} K_{\mathrm{A}}$ Values. $\quad$ S5

Physical Data $\quad$ S6

$\begin{array}{ll}\text { Computational Calculations } & \text { S18 }\end{array}$

$\begin{array}{ll}\text { References } & \text { S51 }\end{array}$

$\begin{array}{ll}\text { Spectra } & \text { S52 }\end{array}$ 


\section{General Information}

Unless otherwise noted, all commercially available compounds were used as provided without further purification. Chemicals used in this manuscript were purchased from Sigma Aldrich, Alfa Aesar, Fluorochem and Carl Roth. Solvents used in reactions were p.A. grade. Solvents for chromatography were technical grade and distilled prior to use. Analytical thin-layer chromatography (TLC) was performed on Macherey-Nagel silica gel aluminum plates with F254 indicator, visualized by irradiation with UV light. Column chromatography was performed using silica gel Merck 60 (particle size $0.040-0.063 \mathrm{~mm}$ or $0.063-0.2 \mathrm{~mm}$ ). Solvent mixtures are understood as volume/volume. ${ }^{1} \mathrm{H}-\mathrm{NMR},{ }^{19} \mathrm{~F}-\mathrm{NMR}$ and ${ }^{13} \mathrm{C}-\mathrm{NMR}$ were recorded on a Varian AV600/AV400, Agilent DD2 400 NMR, Bruker Avance III 300 MHz, Bruker Avance III $400 \mathrm{MHz}$, or Bruker Avance III $600 \mathrm{MHz}$ Cryo NMR spectrometer in $\mathrm{CDCl}_{3} .{ }^{13} \mathrm{C}$-NMR spectra were recorded at $298 \mathrm{~K}$ with proton decoupling. Data are reported in the following order: chemical shift $(\delta)$ in ppm; multiplicities are indicated br (broadened singlet), s (singlet), d (doublet), t (triplet), q (quartet), $\mathrm{m}$ (multiplet); coupling constants $(J)$ are in Hertz $(\mathrm{Hz})$. Data is expressed in parts per million (ppm) downfield shift relative to the internal reference. HRMS data were recorded on a ThermoFisher Scientific LTQ Orbitrap XL using ESI ionization or on a Finnigan MAT 95 using EI ionization at $70 \mathrm{eV}$. IR spectra were recorded on a Perkin Elmer-100 or a ThermoNicolet Avatar 370 FT-IR spectrometer and are reported in terms of frequency of absorption $\left(\mathrm{cm}^{-1}\right)$. Blue light reactions were performed using LEDs purchased from Conrad Electronics: High Power LED-Module, 3 W, 30 lm, $30^{\circ}$, 470 $\mathrm{nm}$, art.nr. $180745-62$ or a custom built blue LED photoreactor. The custom reactor was comprised of a square metal frame $(100 \mathrm{~mm} \times 110 \mathrm{~mm})$ with 14 blue LEDs $(2 \times$ SP-02-V4 LED assembly, each consisting of 7 LXML-PR02-A900 RoyalBlue LUXEON Rebel ES LEDs). Reactions were irradiated from $4 \mathrm{~cm}$, temperature was set to ambient $\left(\sim 27{ }^{\circ} \mathrm{C}\right)$ and cooling was realized with a cooling fan. Reactions using a CFL lamp were performed using a, Philips TORNADO 23W/827 E27 (warm light), $1570 \mathrm{~lm}$.

\section{Important Safety Note}

Handling of diazo compounds should only be done in a well-ventilated fume cupboard using an additional blast shield. No incidents occurred handling of diazoalkanes during the preparation of this manuscript, yet the reader should be aware of carcinogenicity and explosiveness of the herein described diazo compounds. General safety precautions when working with diazomethane and its derivatives should be followed. Any reactions described in this manuscript should not be performed without strict risk assessment and proper safety precautions. 


\section{Experimental Procedures}

\section{General Procedure for NMR Experiments (Chemical Shift Perturbation) - GP 1}

A $0.1 \mathrm{M}$ solution of a $1: 1$ mixture of phenol 10 and methyl phenyldiazoacetate $\mathbf{6 a}$ in $\mathrm{CDCl}_{3}$ was studied by ${ }^{1} \mathrm{H}$ and ${ }^{13} \mathrm{C}$ NMR spectroscopy. As reference a $0.1 \mathrm{M}$ solution of phenol in $\mathrm{CDCl}_{3}$ and a $0.1 \mathrm{M}$ solution of methyl phenyldiazoacetate in $\mathrm{CDCl}_{3}$ was measured using the same NMR spectrometer.

\section{General Procedure for IR Experiments - GP 2}

A 1 : 1 mixture of phenol $\mathbf{1 0}$ and methyl phenyldiazoacetate $\mathbf{6 a}$ was analyzed by IR spectroscopy. As reference methyl phenyldiazoacetate was measured using the same IR spectrometer.

\section{General Procedure for O-H Insertion Reactions - GP 3}

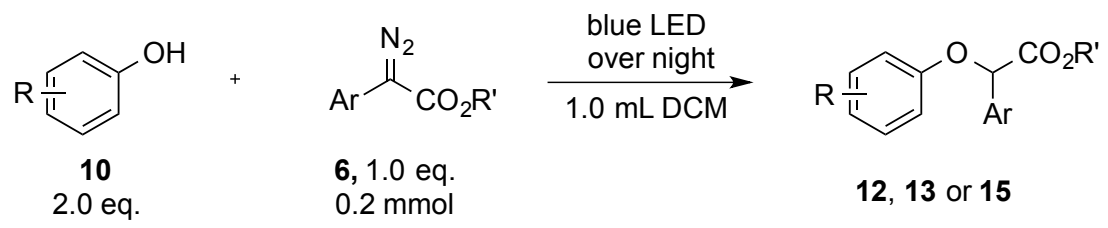

In a $4 \mathrm{~mL}$ reaction vial phenol 10 (2.0 equiv.; $0.4 \mathrm{mmol})$ and the diazo compound $\mathbf{6}$ (1.0 equiv.; $0.2 \mathrm{mmol}$ ) were dissolved in $1.0 \mathrm{~mL} \mathrm{DCM}$ and irradiated with blue LEDs overnight at ambient temperature $\left(\sim 27^{\circ} \mathrm{C}\right)$ or with one single blue LED $\left(3 \mathrm{~W}, 30 \mathrm{~lm}, 30^{\circ}, 470 \mathrm{~nm}\right)$ using a cooling fan. DCM was added to the reaction mixture and the mixture was washed with $1 \mathrm{M}$ aq. $\mathrm{NaOH}$. The organic layer was dried over $\mathrm{MgSO}_{4}$ and concentrated under reduced pressure. The product was obtained after column chromatography using $n$-hexanes : EtOAc or $n$-pentane : $\mathrm{Et}_{2} \mathrm{O}$ as eluent.

\section{Procedure for the Reaction on 1 mmol Scale}

In a $20 \mathrm{~mL}$ reaction vial 4-CN-phenol $\mathbf{1 0 b}(238 \mathrm{mg}$; 2.0 equiv.; $2.0 \mathrm{mmol})$ and the diazo compound 6a (176 mg; 1.0 equiv.; $1.0 \mathrm{mmol}$ ) were dissolved in $10 \mathrm{~mL}$ DCM and irradiated with blue LEDs overnight at ambient temperature $\left(\sim 27^{\circ} \mathrm{C}\right)$ or with one single blue LED (3 $\mathrm{W}, 30 \mathrm{~lm}, 30^{\circ}, 470 \mathrm{~nm}$ ) using a cooling fan. DCM was added to the reaction mixture and the mixture was washed with $1 \mathrm{M}$ aq. $\mathrm{NaOH}$. The organic layer was dried over $\mathrm{MgSO}_{4}$ and concentrated under reduced pressure. The product 12a was obtained after column chromatography using $n$-hexanes : EtOAc or $n$-pentane : $\mathrm{Et}_{2} \mathrm{O}$ as eluent; $206 \mathrm{mg}$ (77\%). 
Data on Chemical Shift Perturbations, Resonant Frequencies, $\mathbf{p} K_{\mathrm{A}}$ Values, Energy of Intramolecular Hydrogen Bond Strength and Yield

Table S1: Data on Chemical Shift Perturbations, Resonant Frequencies and $\mathrm{p} K_{\mathrm{A}}$ of different substituted phenols.

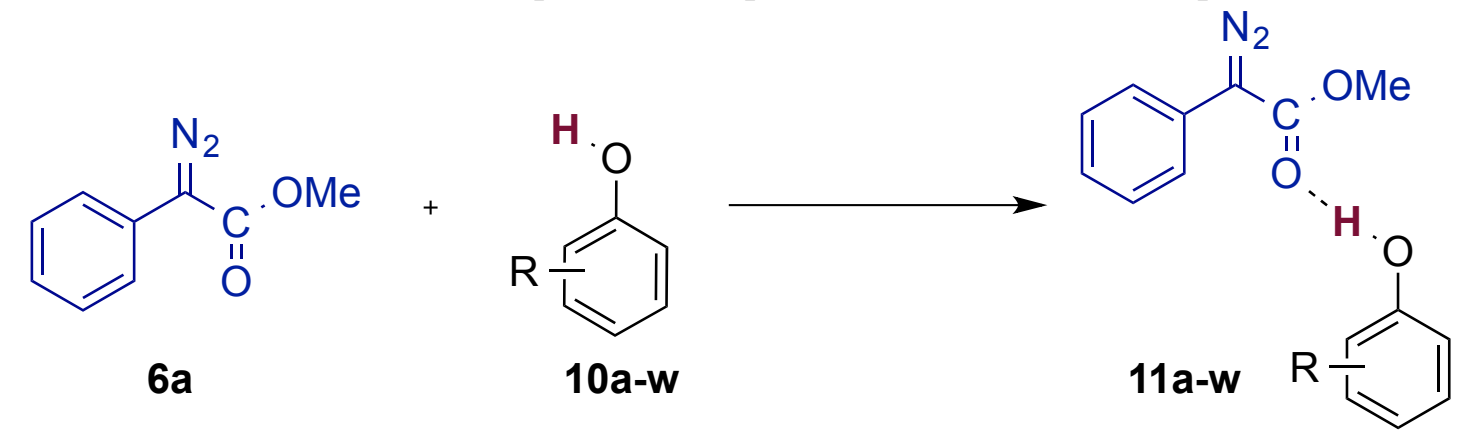

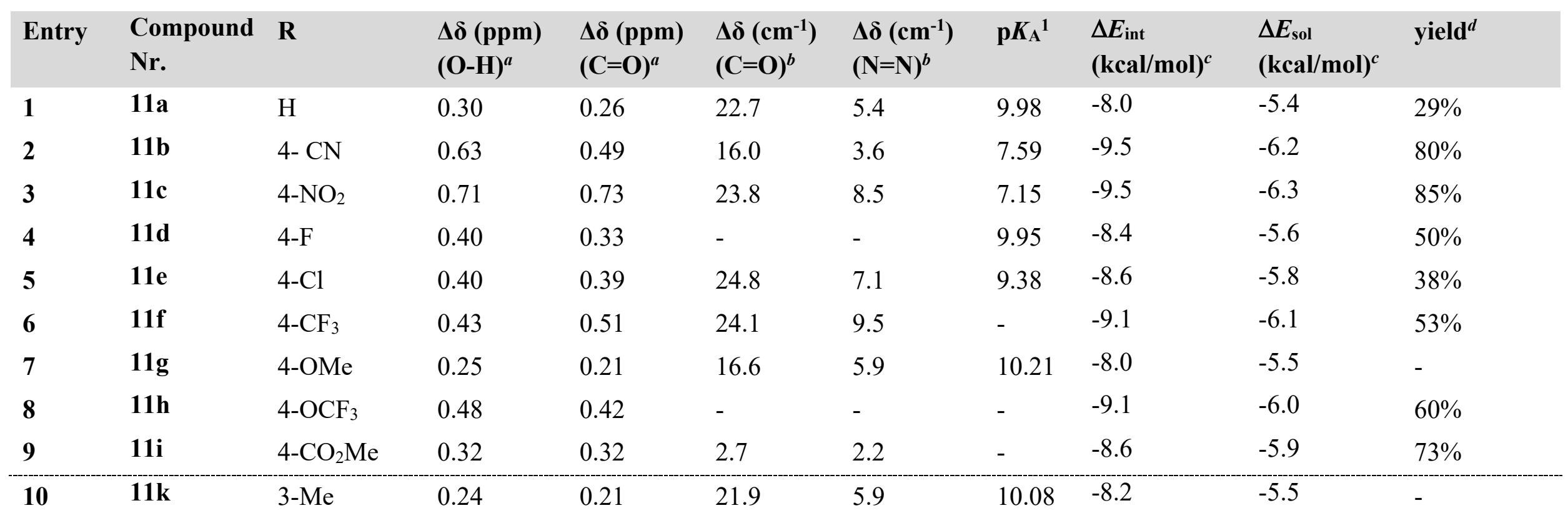




\begin{tabular}{|c|c|c|c|c|c|c|c|c|c|c|}
\hline 11 & 111 & $3-\mathrm{NO}_{2}$ & 0.65 & 0.65 & 26.0 & 5.2 & 8.40 & -10.1 & -6.0 & $85 \%$ \\
\hline 12 & $11 \mathrm{~m}$ & $3-F$ & 0.46 & 0.44 & 25.5 & 9.8 & 9.28 & -8.5 & -5.8 & $47 \%$ \\
\hline 13 & $11 n$ & $3-\mathrm{Cl}$ & 0.52 & 0.44 & - & - & 9.03 & -8.6 & -5.8 & - \\
\hline 14 & 110 & $3-\mathrm{CF}_{3}$ & 0.30 & 0.38 & 22.9 & 8.3 & - & -8.9 & -6.0 & $42 \%$ \\
\hline 15 & $11 p$ & 3-OMe & 0.24 & 0.24 & 21.5 & 6.0 & 9.65 & -8.4 & -5.9 & - \\
\hline 16 & $11 q$ & $3-\mathrm{CO}_{2} \mathrm{Me}$ & 0.17 & 0.25 & 16.0 & 5.2 & - & -9.1 & -5.1 & - \\
\hline 17 & $11 \mathrm{r}$ & 2-Me & 0.23 & 0.16 & 20.0 & 5.8 & 10.29 & -8.0 & -5.5 & - \\
\hline 18 & $11 \mathrm{~s}$ & $2-\mathrm{CN}$ & 0.35 & 0.38 & 7.9 & 10.5 & - & -7.5 & -6.6 & $41 \%$ \\
\hline 19 & $11 \mathrm{j}$ & $2-\mathrm{NO}_{2}$ & 0.00 & 0.00 & 3.0 & 3.0 & 7.23 & -4.5 & -2.8 & $37 \%$ \\
\hline 20 & $11 t$ & $2-\mathrm{F}$ & 0.16 & 0.13 & 19.6 & 7.8 & & -6.1 & -4.3 & - \\
\hline 21 & $11 u$ & $2-\mathrm{Cl}$ & 0.03 & 0.07 & 21.3 & 6.7 & 8.56 & -6.2 & -5.5 & $20 \%$ \\
\hline 22 & $11 v$ & 2-OMe & 0.02 & 0.02 & 16.0 & 5.7 & - & -5.7 & -4.7 & - \\
\hline 23 & $11 w$ & $2-\mathrm{CO}_{2} \mathrm{Me}$ & 0.00 & 0.01 & - & - & - & -4.0 & -2.2 & no reaction \\
\hline $24^{[\mathrm{e}]}$ & $11 x$ & $\mathrm{H}$ & 0.01 & - & - & - & 9.98 & & & $43 \%$ \\
\hline $25^{[\mathrm{e}]}$ & $11 y$ & $4-\mathrm{CN}$ & 0.01 & - & - & - & 7.59 & & & - \\
\hline
\end{tabular}

conditions: ${ }^{a}$ a $0.1 \mathrm{M}$ solution of a $1: 1$ mixture of phenole and methyl phenyldiazoacetate in $\mathrm{CDCl}_{3} ;{ }^{b} \mathrm{a} 1: 1$ mixture of phenol and methyl phenyldiazoacetate; 'Interaction energies calculated at the MP2/6-311+G(d,p)//B3LYP/6-31G(d) level with BSSE correction; ${ }^{d}$ isolated yield under optimized reaction conditions; ${ }^{e}$ (1-diazo-2,2,2-trifluoroethyl)benzene instead of methyl phenyldiazoacetate. 


\section{Chemical Shift Perturbation vs. Experimental $p K_{\mathrm{A}}$ Values}

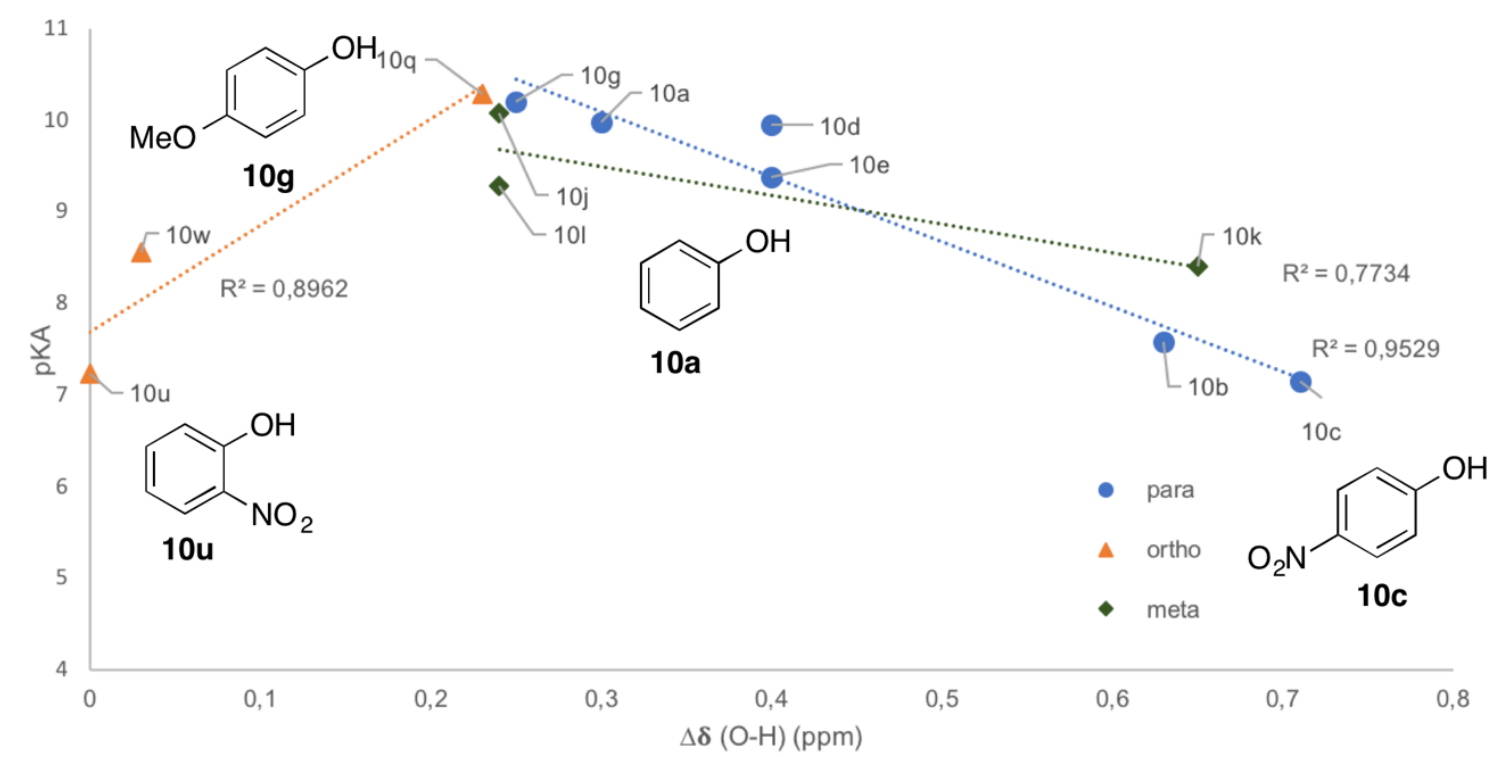

Scheme S1: Chemical shift perturbation $v s$. experimental $\mathrm{p} K_{\mathrm{A}}$ values. $\mathrm{p} K \mathrm{~A}$ values from: Liptak, M. D.; Gross, K. C.; Seybold, P. G.; Feldgus, S.; Shields, G. C. Absolute pKa Determinations for Substituted Phenols. J. Am. Chem. Soc. 2002, 124, 6421-6427 


\section{Physical Data}

\section{Methyl 2-(4-cyanophenoxy)-2-phenylacetate (12a)}<smiles>CC(=O)C(Oc1ccc(C#N)cc1)c1ccccc1</smiles>

Compound 12a was prepared according to the general procedure GP-3 and was obtained after column chromatography ( $n$-hexane : EtOAc 20:1 $\rightarrow$ 9:1); 80\% (43 mg); colorless oil.

${ }^{1}$ H NMR (400 MHz, Chloroform- $d$ ): $\delta=7.92(\mathrm{dd}, J=8.1,1.7 \mathrm{~Hz}, 1 \mathrm{H}), 7.69-7.60(\mathrm{~m}, 2 \mathrm{H}), 7.55-7.49$ (m, 1H), $7.46-7.36(\mathrm{~m}, 3 \mathrm{H}), 7.16-7.09(\mathrm{~m}, 1 \mathrm{H}), 7.02(\mathrm{dd}, J=8.4,1.1 \mathrm{~Hz}, 1 \mathrm{H}), 5.79(\mathrm{~s}, 1 \mathrm{H}), 3.75$ (s, 3H) $\mathrm{ppm}$.

${ }^{13}$ C NMR (101MHz, Chloroform- $d$ ): $\delta=169.3,160.3,134.2,134.1,129.4,129.0,127.0,118.8,116.0$, $105.2,78.5,52.9 \mathrm{ppm}$.

HRMS (ESI): $m / z$ : $[\mathrm{M}+\mathrm{Na}]^{+}$Calcd. for $\mathrm{C}_{16} \mathrm{H}_{13} \mathrm{O}_{3} \mathrm{NNa}$ : 290.0787; Found: 290.0787 .

IR (neat): 3487, 3104, 3067, 3002, 2952, 2844, 2681, 2589, 2546, 2497, 2442, 2342, 2221, 2094, 1907, $1807,1750,1599,1495,1454,1431,1340,1293,1246,1218,1169,1111,1081,1053,990,942,912$, $828,787,745,692 \mathrm{~cm}^{-1}$.

\section{Ethyl 2-(4-cyanophenoxy)-2-phenylacetate (12b)}<smiles>CCOC(=O)C(Oc1ccc(C#N)cc1)c1ccc(C#N)cc1</smiles>

Compound 12b was prepared according to the general procedure GP-3 and was obtained after column chromatography (n-pentane : $\left.\mathrm{Et}_{2} \mathrm{O} 20: 1 \rightarrow 9: 1\right) ; 64 \%$ (36 mg); colorless oil.

${ }^{1}$ H NMR $(600 \mathrm{MHz}$, Chloroform- $d$ ): $\delta=7.63-7.56(\mathrm{~m}, 4 \mathrm{H}), 7.47-7.41(\mathrm{~m}, 3 \mathrm{H}), 7.05-7.01(\mathrm{~m}, 2 \mathrm{H})$, $5.68(\mathrm{~s}, 1 \mathrm{H}), 4.32-4.16(\mathrm{~m}, 2 \mathrm{H}), 1.23(\mathrm{t}, J=7.1 \mathrm{~Hz}, 3 \mathrm{H}) \mathrm{ppm}$.

${ }^{13}$ C NMR (151 MHz, Chloroform- $d$ ): $\delta=168.8,160.4,134.3,134.0,129.3,128.9,127.0,118.8,116.0$, 105.2, 78.6, 62.0, $14.0 \mathrm{ppm}$.

HRMS (ESI): $m / z$ : $[\mathrm{M}]^{+}$Calcd. for $\mathrm{C}_{17} \mathrm{H}_{16} \mathrm{O}_{3} \mathrm{~N}: 282.1124$; Found: 282.1120 .

IR (KBr): 3845, 3206, 3068, 2983, 2931, 2548, 2325, 2225, 2093, 1903, 1746, 1603, 1503, 1454, 1370, $1296,1245,1175,1086,1052,1024,919,835,722,696 \mathrm{~cm}^{-1}$.

\section{Allyl 2-(4-cyanophenoxy)-2-phenylacetate (12c)}

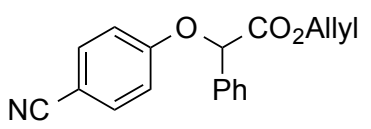

Compound 12c was prepared according to the general procedure GP-3 and was obtained after column chromatography ( $n$-pentane : $\left.\mathrm{Et}_{2} \mathrm{O} 20: 1 \rightarrow 9: 1\right) ; 70 \%(41 \mathrm{mg})$; colorless oil.

${ }^{1}$ H NMR (600 MHz, Chloroform- $d$ ): $\delta=7.63-7.57(\mathrm{~m}, 4 \mathrm{H}), 7.48-7.41(\mathrm{~m}, 3 \mathrm{H}), 7.07-7.01(\mathrm{~m}, 2 \mathrm{H})$, $5.87-5.80(\mathrm{~m}, 1 \mathrm{H}), 5.72(\mathrm{~s}, 1 \mathrm{H}), 5.24-5.19(\mathrm{~m}, 2 \mathrm{H}), 4.81-4.58(\mathrm{~m}, 2 \mathrm{H}) \mathrm{ppm}$.

${ }^{13}$ C NMR (151 MHz, Chloroform- $d$ ): $\delta=168.5,160.3,134.2,134.1,131.0,129.4,129.0,127.1,119.0$, $118.8,116.1,105.2,78.5,66.2 \mathrm{ppm}$.

HRMS (ESI): $m / z$ : $[\mathrm{M}+\mathrm{Na}]^{+}$Calcd. for $\mathrm{C}_{18} \mathrm{H}_{15} \mathrm{O}_{3} \mathrm{NNa}$ : 316.0944 ; Found: 316.0937 .

IR (KBr): 3491, 3071, 2941, 2551, 2226, 2171, 1900, 1749, 1648, 1603, 1503, 1453, 1420, 1367, 1296, $1245,1172,1085,1051,985,933,834,721,696 \mathrm{~cm}^{-1}$. 


\section{Benzyl 2-(4-cyanophenoxy)-2-phenylacetate (12d)}<smiles>N#Cc1ccc(OC(C(=O)Oc2ccccc2)c2ccccc2)cc1</smiles>

Compound 12d was prepared according to the general procedure GP-3 and was obtained after column chromatography ( $n$-pentane $\left.: \mathrm{Et}_{2} \mathrm{O} 20: 1 \rightarrow 9: 1\right) ; 71 \%$ (49 mg); colorless oil.

${ }^{1}$ H NMR (600 MHz, Chloroform- $\left.d\right): \delta=7.60-7.52(\mathrm{~m}, 4 \mathrm{H}), 7.47-7.41(\mathrm{~m}, 3 \mathrm{H}), 7.36-7.30(\mathrm{~m}, 3 \mathrm{H})$, $7.23-7.18(\mathrm{~m}, 2 \mathrm{H}), 7.01-6.95(\mathrm{~m}, 2 \mathrm{H}), 5.72(\mathrm{~s}, 1 \mathrm{H}), 5.23(\mathrm{~d}, J=12.2 \mathrm{~Hz}, 1 \mathrm{H}), 5.16(\mathrm{~d}, J=12.2 \mathrm{~Hz}$, 1H) $\mathrm{ppm}$.

${ }^{13}$ C NMR (151 MHz, Chloroform- $\left.d\right): \delta=168.7,160.3,134.8,134.1,134.0,129.4,129.0,128.5,128.2$, 127.1, 118.8, 116.0, 105.2, 78.6, 67.5 ppm.

HRMS (ESI): $m / z$ : $[\mathrm{M}+\mathrm{Na}]^{+}$Calcd. for $\mathrm{C}_{22} \mathrm{H}_{17} \mathrm{O}_{3} \mathrm{NNa}$ : 366.1100; Found: 366.1093 .

IR (KBr): 3207, 3036, 2953, 2326, 2225, 2089, 2014, 1958, 1897, 1750, 1603, 1502, 1454, 1419, 1377, $1297,1245,1170,1112,1083,1052,965,913,834,733,696 \mathrm{~cm}^{-1}$.

\section{Cyclohexyl 2-(4-cyanophenoxy)-2-phenylacetate (12e)}<smiles>N#Cc1ccc(OC(C(=O)O)c2ccccc2)cc1</smiles>

Compound 12e was prepared according to the general procedure GP-3 and was obtained after column chromatography (n-pentane : $\left.\mathrm{Et}_{2} \mathrm{O} 20: 1 \rightarrow 9: 1\right) ; 69 \%(46 \mathrm{mg})$; colorless oil.

${ }^{1}$ H NMR (600 MHz, Chloroform- $\left.d\right)$ : $\delta=7.62-7.56(\mathrm{~m}, 4 \mathrm{H}), 7.47-7.39(\mathrm{~m}, 3 \mathrm{H}), 7.05-7.01(\mathrm{~m}, 2 \mathrm{H})$, $5.66(\mathrm{~s}, 1 \mathrm{H}), 4.88-4.81(\mathrm{~m}, 1 \mathrm{H}), 1.88-1.80(\mathrm{~m}, 1 \mathrm{H}), 1.75-1.67(\mathrm{~m}, 1 \mathrm{H}), 1.66-1.62(\mathrm{~m}, 1 \mathrm{H}), 1.55-$ $1.45(\mathrm{~m}, 3 \mathrm{H}), 1.42-1.34(\mathrm{~m}, 1 \mathrm{H}), 1.33-1.22(\mathrm{~m}, 3 \mathrm{H}) \mathrm{ppm}$.

${ }^{13}$ C NMR (151 MHz, Chloroform- $\left.d\right): \delta=168.3,160.5,134.5,134.0,129.3,128.9,127.0,118.9,116.0$, 105.1, 78.7, 74.4, 31.3, 30.9, 25.1, 23.3, $23.1 \mathrm{ppm}$.

HRMS (ESI): $m / z$ : $[\mathrm{M}+\mathrm{Na}]^{+}$Calcd. for $\mathrm{C}_{21} \mathrm{H}_{21} \mathrm{O}_{3} \mathrm{NNa}$ : 358.1413; Found: 358.1405 .

IR (KBr): 3482, 3068, 2936, 2860, 2665, 2549, 2324, 2225, 2160, 2087, 1987, 1900, 1742, 1603, 1503 , $1452,1246,1176,1117,1084,1050,1011,965,912,834,724,696 \mathrm{~cm}^{-1}$.

\section{Methyl 2-(4-cyanophenoxy)-2-(4-fluorophenyl)acetate (12f)}<smiles>CC(=O)C(Oc1ccc(C#N)cc1)c1ccc(F)cc1</smiles>

Compound 12f was prepared according to the general procedure GP-3 and was obtained after column chromatography (n-pentane : $\left.\mathrm{Et}_{2} \mathrm{O} 20: 1 \rightarrow 9: 1\right) ; 79 \%(45 \mathrm{mg})$; colorless oil.

${ }^{1}$ H NMR (600 MHz, Chloroform- $d$ ): $\delta=7.63-7.59(\mathrm{~m}, 2 \mathrm{H}), 7.58-7.54(\mathrm{~m}, 2 \mathrm{H}), 7.16-7.11(\mathrm{~m}, 2 \mathrm{H})$, $7.02-6.99(\mathrm{~m}, 2 \mathrm{H}), 5.68(\mathrm{~s}, 1 \mathrm{H}), 3.78(\mathrm{~s}, 3 \mathrm{H}) \mathrm{ppm}$.

${ }^{13}$ C NMR (151 MHz, Chloroform- $d$ ): $\delta=169.2,163.2(\mathrm{~d}, J=249.4 \mathrm{~Hz}), 160.2,134.1,130.1(\mathrm{~d}, J=2.7$ $\mathrm{Hz}), 128.9(\mathrm{~d}, J=8.5 \mathrm{~Hz}), 118.7,116.1(\mathrm{~d}, J=21.9 \mathrm{~Hz}), 116.0,105.4,77.8,52.9 \mathrm{ppm}$.

${ }^{19}$ F NMR (565 MHz, Chloroform- $d$ ): $\delta=-111.46--111.57(\mathrm{~m}) \mathrm{ppm}$.

HRMS (ESI): $m / z$ : $[\mathrm{M}+\mathrm{Na}]^{+}$Calcd. for $\mathrm{C}_{16} \mathrm{H}_{12} \mathrm{O}_{3} \mathrm{NFNa}$ : 308.0693; Found: 308.0688 .

IR (KBr): 3494, 3076, 2956, 2549, 2226, 2153, 1901, 1751, 1603, 1505, 1438, 1296, 1224, 1170, 1104, $1057,1013,976,899,832,759,733,697 \mathrm{~cm}^{-1}$. 


\section{Methyl 2-(4-chlorophenyl)-2-(4-cyanophenoxy)acetate (12g)}<smiles>CC(=O)C(Oc1ccc(C#N)cc1)c1ccc(Cl)cc1</smiles>

Compound 12g was prepared according to the general procedure GP-3 and was obtained after column chromatography ( $n$-pentane : $\left.\mathrm{Et}_{2} \mathrm{O} 20: 1 \rightarrow 9: 1\right) ; 86 \%$ (52 mg); colorless oil.

${ }^{1}$ H NMR (600 MHz, Chloroform- $d$ ): $\delta=7.66-7.58(\mathrm{~m}, 2 \mathrm{H}), 7.55-7.48(\mathrm{~m}, 2 \mathrm{H}), 7.45-7.40(\mathrm{~m}, 2 \mathrm{H})$, $7.06-6.94(\mathrm{~m}, 2 \mathrm{H}), 5.67(\mathrm{~s}, 1 \mathrm{H}), 3.78(\mathrm{~s}, 3 \mathrm{H}) \mathrm{ppm}$.

${ }^{13}$ C NMR (151 MHz, Chloroform- $d$ ): $\delta=169.0,160.1,135.5,134.1,132.7,129.2,128.3,118.7,116.0$, $105.5,77.8,53.0 \mathrm{ppm}$.

HRMS (ESI): $m / z:[\mathrm{M}+\mathrm{Na}]^{+}$Calcd. for $\mathrm{C}_{16} \mathrm{H}_{12} \mathrm{O}_{3} \mathrm{NClNa}$ : 324.0397; Found: 324.0397.

IR (KBr): 3493, 3071, 3006, 2955, 2852, 2549, 2226, 2161, 1907, 1751, 1603, 1500, 1438, 1412, 1342, $1294,1244,1211,1172,1088,1060,1013,976,899,830,770,730 \mathrm{~cm}^{-1}$.

\section{Methyl 2-(4-bromophenyl)-2-(4-cyanophenoxy)acetate (12h)}<smiles>CC(=O)C(Oc1ccc(C#N)cc1)c1ccc(Br)cc1</smiles>

Compound 12h was prepared according to the general procedure GP-3 and was obtained after column chromatography (n-pentane : $\left.\mathrm{Et}_{2} \mathrm{O} 20: 1 \rightarrow 9: 1\right) ; 76 \%$ (53 mg); colorless oil.

${ }^{1}$ H NMR $(600 \mathrm{MHz}$, Chloroform- $d): \delta=7.63-7.59(\mathrm{~m}, 2 \mathrm{H}), 7.59-7.55(\mathrm{~m}, 2 \mathrm{H}), 7.48-7.43(\mathrm{~m}, 2 \mathrm{H})$, $7.02-6.98(\mathrm{~m}, 2 \mathrm{H}), 5.65(\mathrm{~s}, 1 \mathrm{H}), 3.77(\mathrm{~s}, 3 \mathrm{H}) \mathrm{ppm}$.

${ }^{13}$ C NMR (151 MHz, Chloroform- $d$ ): $\delta=168.9,160.1,134.1,133.2,132.2,128.6,123.7,118.7,116.0$, $105.5,77.8,53.0 \mathrm{ppm}$.

HRMS (ESI): $m / z$ : $[\mathrm{M}]^{+}$Calcd. for $\mathrm{C}_{16} \mathrm{H}_{13} \mathrm{BrNO}_{3}{ }^{+}: 346.0073$; Found: 346.0072 .

IR (KBr): 3493, 3071, 2954, 2326, 2226, 2091, 1908, 1751, 1602, 1502, 1437, 1342, 1293, 1244, 1172, $1112,1064,1010,976,907,766,730 \mathrm{~cm}^{-1}$.

Methyl 2-(4-cyanophenoxy)-2-(4-methoxyphenyl)acetate (12i)<smiles>COc1ccc(C(Oc2ccc(C#N)cc2)C(C)=O)cc1</smiles>

Compound 12i was prepared according to the general procedure GP-3 and was obtained after column chromatography (n-pentane : $\left.\mathrm{Et}_{2} \mathrm{O} 20: 1 \rightarrow 9: 1\right) ; 84 \%$ (50 mg); colorless oil.

${ }^{1}$ H NMR $(600 \mathrm{MHz}$, Chloroform- $d): \delta=7.62-7.57(\mathrm{~m}, 2 \mathrm{H}), 7.52-7.45(\mathrm{~m}, 2 \mathrm{H}), 7.03-6.98(\mathrm{~m}, 2 \mathrm{H})$, $6.97-6.93(\mathrm{~m}, 2 \mathrm{H}), 5.64(\mathrm{~s}, 1 \mathrm{H}), 3.84(\mathrm{~s}, 3 \mathrm{H}), 3.77(\mathrm{~s}, 3 \mathrm{H}) \mathrm{ppm}$.

${ }^{13}$ C NMR (151 MHz, Chloroform- $d$ ): $\delta=169.6,160.49,160.45,134.0,128.5,126.2,118.8,116.0$, 114.4, 105.1, 78.1, 55.3, $52.8 \mathrm{ppm}$.

HRMS (ESI): $m / z$ : [M] $]^{+}$Calcd. for $\mathrm{C}_{17} \mathrm{H}_{15} \mathrm{NNaO}_{4}$ : 320.0893; Found: 320.0892 .

IR (KBr): 3006, 2955, 2841, 2551, 2225, 2020, 1901, 1750, 1604, 1506, 1440, 1299, 1241, 1172, 1114 , $1031,976,909,832,757,732 \mathrm{~cm}^{-1}$. 
Methyl 2-(3-chlorophenyl)-2-(4-cyanophenoxy)acetate (12j)<smiles>CC(=O)C(Oc1ccc(C#N)cc1)c1cccc(Cl)c1</smiles>

Compound $\mathbf{1 2} \mathbf{j}$ was prepared according to the general procedure GP-3 and was obtained after column chromatography (n-pentane : $\left.\mathrm{Et}_{2} \mathrm{O} 20: 1 \rightarrow 9: 1\right) ; 50 \%$ (30 mg); colorless oil.

${ }^{1}$ H NMR (600 MHz, Chloroform- $d$ ): $\delta=7.65-7.58(\mathrm{~m}, 3 \mathrm{H}), 7.48-7.44(\mathrm{~m}, 1 \mathrm{H}), 7.42-7.35(\mathrm{~m}, 2 \mathrm{H})$, $7.07-6.97(\mathrm{~m}, 2 \mathrm{H}), 5.66(\mathrm{~s}, 1 \mathrm{H}), 3.79(\mathrm{~s}, 3 \mathrm{H}) \mathrm{ppm}$.

${ }^{13}$ C NMR (151 MHz, Chloroform- $d$ ): $\delta=168.8,160.0,136.1,135.0,134.1,130.2,129.6,127.1,125.1$, 118.7, 116.0, 105.6, 77.7, $53.1 \mathrm{ppm}$.

HRMS (ESI): $m / z$ : $[\mathrm{M}]^{+}$Calcd. for $\mathrm{C}_{16} \mathrm{H}_{13} \mathrm{ClNO}_{3}$ : 302.0578; Found: 302.0579.

IR (KBr): 3492, 3072, 2955, 2851, 2549, 2226, 2161, 2020, 1903, 1752, 1602, 1578, 1434, 1296, 1243, $1208,1172,1100,1011,979,909,802,682 \mathrm{~cm}^{-1}$.

Methyl 2-(4-cyanophenoxy)-2-(3-methoxyphenyl)acetate (12k)<smiles>COc1cccc(C(Oc2ccc(C#N)cc2)C(C)=O)c1</smiles>

Compound 12k was prepared according to the general procedure GP-3 and was obtained after column chromatography (n-pentane : $\left.\mathrm{Et}_{2} \mathrm{O} 20: 1 \rightarrow 9: 1\right) ; 77 \%$ (46 mg); colorless oil.

${ }^{1}$ H NMR $(600 \mathrm{MHz}$, Chloroform- $d$ ): $\delta=7.62-7.55(\mathrm{~m}, 2 \mathrm{H}), 7.35(\mathrm{t}, J=8.0 \mathrm{~Hz}, 1 \mathrm{H}), 7.18-7.08$ (m, $2 \mathrm{H}), 7.05-7.00(\mathrm{~m}, 2 \mathrm{H}), 6.96-6.93(\mathrm{~m}, 1 \mathrm{H}), 5.66(\mathrm{~s}, 1 \mathrm{H}), 3.85(\mathrm{~s}, 3 \mathrm{H}), 3.78(\mathrm{~s}, 3 \mathrm{H}) \mathrm{ppm}$.

${ }^{13}$ C NMR (151 MHz, Chloroform- $d$ ): $\delta=169.2,160.3,160.0,135.6,134.1,130.0,119.3,118.8,116.0$, $114.9,112.5,105.3,78.4,55.3,52.9 \mathrm{ppm}$.

HRMS (ESI): $m / z$ : $[\mathrm{M}+\mathrm{Na}]^{+}$Calcd. for $\mathrm{C}_{17} \mathrm{H}_{15} \mathrm{NNaO}_{4}$ : 320.0893; Found: 320.0888 .

IR (KBr): 3485, 3006, 2953, 2840, 2225, 2021, 1924, 1750, 1601, 1500, 1436, 1244, 1206, 1171, 1043, $835,784,749,689 \mathrm{~cm}^{-1}$.

Methyl 2-(4-cyanophenoxy)-2-(2-fluorophenyl)acetate (12I)<smiles>CC(=O)C(Oc1ccc(C#N)cc1)c1ccccc1F</smiles>

Compound 12I was prepared according to the general procedure GP-3 and was obtained after column chromatography (n-pentane : $\left.\mathrm{Et}_{2} \mathrm{O} 20: 1 \rightarrow 9: 1\right) ; 44 \%(25 \mathrm{mg})$; colorless oil.

${ }^{1}$ H NMR (600 MHz, Chloroform-d): $\delta=7.64-7.56(\mathrm{~m}, 3 \mathrm{H}), 7.45-7.37$ (m, 1H), 7.23 (t, $J=7.6 \mathrm{~Hz}$, $1 \mathrm{H}), 7.19-7.15(\mathrm{~m}, 1 \mathrm{H}), 7.06-7.02(\mathrm{~m}, 2 \mathrm{H}), 6.07(\mathrm{~s}, 1 \mathrm{H}), 3.80(\mathrm{~s}, 3 \mathrm{H}) \mathrm{ppm}$.

${ }^{13}$ C NMR (151 MHz, Chloroform- $d$ ): $\delta=168.7,160,17,160.15$ (d, $\left.J=249.3 \mathrm{~Hz}\right), 134.1,131.3(\mathrm{~d}, J=$ $8.4 \mathrm{~Hz}), 128.5$ (d, $J=2.6 \mathrm{~Hz}), 124.9$ (d, $J=3.6 \mathrm{~Hz}), 121.9$ (d, $J=13.4 \mathrm{~Hz}), 118.7,116.0,115.9,115.8$, $105.5,71.4(\mathrm{~d}, J=3.6 \mathrm{~Hz}) \mathrm{ppm}$.

${ }^{19}$ F NMR (565 MHz, Chloroform- $d$ ): $\delta=-117.84--117.90(\mathrm{~m}) \mathrm{ppm}$.

HRMS (ESI): $m / z$ : $[\mathrm{M}]^{+}$Calcd. for $\mathrm{C}_{16} \mathrm{H}_{13} \mathrm{O}_{3} \mathrm{NF}: 286.0874$; Found: 286.0868.

IR (KBr): 3508, 3073, 2956, 2849, 2326, 2226, 2095, 2020, 1990, 1899, 1754, 1602, 1500, 1455, 1294, $1236,1173,1103,1056,978,895,834,758,700 \mathrm{~cm}^{-1}$. 


\section{Methyl 2-(2-chlorophenyl)-2-(4-cyanophenoxy)acetate (12m)}<smiles>CC(=O)C(Oc1ccc(C#N)cc1)c1ccccc1Cl</smiles>

Compound 12m was prepared according to the general procedure GP-3 and was obtained after column chromatography (n-pentane : $\left.\mathrm{Et}_{2} \mathrm{O} 20: 1 \rightarrow 9: 1\right) ; 35 \%(21 \mathrm{mg})$; colorless oil.

${ }^{1}$ H NMR (600 MHz, Chloroform- $d$ ): $\delta=7.63-7.58(\mathrm{~m}, 3 \mathrm{H}), 7.50-7.45(\mathrm{~m}, 1 \mathrm{H}), 7.39-7.32(\mathrm{~m}, 2 \mathrm{H})$, $7.07-7.00(\mathrm{~m}, 2 \mathrm{H}), 6.22(\mathrm{~s}, 1 \mathrm{H}), 3.81(\mathrm{~s}, 3 \mathrm{H}) \mathrm{ppm}$.

${ }^{13}$ C NMR (151 MHz, Chloroform- $\left.d\right): \delta=168.6,160.1,134.1,133.5,132.4,130.6,129.9,128.6,127.6$, $118.7,116.1,105.4,74.7,53.0 \mathrm{ppm}$.

HRMS (ESI): $m / z:[\mathrm{M}+\mathrm{Na}]^{+}$Calcd. for $\mathrm{C}_{16} \mathrm{H}_{12} \mathrm{O}_{3} \mathrm{NClNa}$ : 324.0397; Found: 324.0390 .

IR (KBr): 3502, 3071, 2955, 2849, 2549, 2547, 2326, 2226, 2155, 1906, 1753, 1602, 1503, 1432, 1439, $1343,1295,1242,1173,1128,1059,979,909,834,754 \mathrm{~cm}^{-1}$.

\section{Methyl 2-(4-cyanophenoxy)-2-(naphthalen-2-yl)acetate (12n)}<smiles>CC(=O)C(Oc1ccc(C#N)cc1)c1ccc2ccccc2c1</smiles>

Compound 120 was prepared according to the general procedure GP-3 and was obtained after column chromatography (n-pentane : $\left.\mathrm{Et}_{2} \mathrm{O} 20: 1 \rightarrow 9: 1\right) ; 58 \%(37 \mathrm{mg})$; colorless oil.

${ }^{1}$ H NMR (600 MHz, Chloroform- $d$ ): $\delta=8.07-8.01(\mathrm{~m}, 1 \mathrm{H}), 7.95-7.86(\mathrm{~m}, 3 \mathrm{H}), 7.67(\mathrm{dd}, J=8.5,1.8$ $\mathrm{Hz}, 1 \mathrm{H}), 7.62-7.58(\mathrm{~m}, 2 \mathrm{H}), 7.57-7.54(\mathrm{~m}, 2 \mathrm{H}), 7.09-7.05(\mathrm{~m}, 2 \mathrm{H}), 5.87(\mathrm{~s}, 1 \mathrm{H}), 3.78(\mathrm{~s}, 3 \mathrm{H}) \mathrm{ppm}$.

${ }^{13}$ C NMR (151 MHz, Chloroform- $d$ ): $\delta=169.3,160.3,134.1,133.6,133.1,131.6,129.0,128.2,127.8$, $126.9,126.8,126.7,124.0,118.8,116.1,105.3,78.7,52.9 \mathrm{ppm}$.

HRMS (ESI): $m / z$ : [M + Na] $]^{+}$Calcd. for $\mathrm{C}_{20} \mathrm{H}_{15} \mathrm{O}_{3} \mathrm{NNa}$ : 340.0944; Found: 340.0937 .

IR (KBr): 3491, 3058, 2954, 2548, 2226, 2160, 2027, 1912, 1749, 1602, 1504, 1437, 1337, 1243, 1172, $1122,1059,957,908,830,730,664 \mathrm{~cm}^{-1}$.

\section{Methyl 2-(4-cyanophenoxy)-2-(naphthalen-1-yl)acetate (120)}<smiles>CC(=O)C(Oc1ccc(C#N)cc1)c1cccc2ccccc12</smiles>

Compound 12n was prepared according to the general procedure GP-3 and was obtained after column chromatography ( $n$-pentane : $\left.\mathrm{Et}_{2} \mathrm{O} 20: 1 \rightarrow 9: 1\right) ; 49 \%(31 \mathrm{mg})$; colorless oil.

${ }^{1}$ H NMR $(600 \mathrm{MHz}$, Chloroform- $d$ ): $\delta=8.34-8.22(\mathrm{~m}, 1 \mathrm{H}), 8.00-7.87(\mathrm{~m}, 2 \mathrm{H}), 7.75(\mathrm{dd}, J=7.2 \mathrm{~Hz}$, $1 \mathrm{H}), 7.65-7.61(\mathrm{~m}, 1 \mathrm{H}), 7.60-7.56(\mathrm{~m}, 3 \mathrm{H}), 7.54-7.50(\mathrm{~m}, 1 \mathrm{H}), 7.06-7.02(\mathrm{~m}, 2 \mathrm{H}), 6.37(\mathrm{~s}, 1 \mathrm{H})$, $3.77(\mathrm{~s}, 3 \mathrm{H}) \mathrm{ppm}$.

${ }^{13}$ C NMR (151 MHz, Chloroform- $d$ ): $\delta=169.3,160.4,134.1,134.0,130.5,130.2,130.1,129.1,127.1$, $126.5,126.2,125.3,123.3,118.8,116.2,105.3,76.6,52.9 \mathrm{ppm}$.

HRMS (ESI): $m / z$ : [M + Na] $]^{+}$Calcd. for $\mathrm{C}_{20} \mathrm{H}_{15} \mathrm{NNaO}_{3}$ : 340.0944; Found: 340.0939 .

IR (KBr): 3492, 3056, 2954, 2547, 2225, 2160, 2018, 1902, 1750, 1602, 1504, 1437, 1339, 1293, 1240, $1171,1052,974,910,834,730 \mathrm{~cm}^{-1}$. 
Methyl 2-(benzo[d][1,3]dioxol-5-yl)-2-(4-cyanophenoxy)acetate (12p)<smiles>CC(=O)C(Oc1ccc(C#N)cc1)c1ccc2c(c1)OCO2</smiles>

Compound 12p was prepared according to the general procedure GP-3 and was obtained after column chromatography ( $n$-pentane : $\left.\mathrm{Et}_{2} \mathrm{O} 20: 1 \rightarrow 9: 1\right) ; 74 \%$ (46 mg); colorless oil.

${ }^{1}$ H NMR (600 MHz, Chloroform- $d$ ): $\delta=7.62-7.57(\mathrm{~m}, 2 \mathrm{H}), 7.07-6.97(\mathrm{~m}, 4 \mathrm{H}), 6.84(\mathrm{~d}, J=8.0 \mathrm{~Hz}$, $1 \mathrm{H}), 6.01(\mathrm{~s}, 1 \mathrm{H}), 5.59(\mathrm{~s}, 2 \mathrm{H}), 3.78(\mathrm{~s}, 3 \mathrm{H}) \mathrm{ppm}$.

${ }^{13}$ C NMR (151 MHz, Chloroform- $d$ ): $\delta=169.3,160.2,148.6,148.3,134.1,127.8,121.2,118.8,116.0$, 108.6, 107.3, 105.2, 101.5, 78.2, $52.9 \mathrm{ppm}$.

HRMS (ESI): $m / z$ : $[\mathrm{M}+\mathrm{Na}]^{+}$Calcd. for $\mathrm{C}_{17} \mathrm{H}_{13} \mathrm{O}_{5} \mathrm{NNa}$ : 334.0685; Found: 334.0679 .

IR (KBr): 3491, 3005, 2956, 2902, 2781, 2548, 2226, 2162, 2038, 1860, 1750, 1603, 1499, 1444, 1375 , $1340,1296,1239,1172,1102,1036,926,865,833,810,761,729 \mathrm{~cm}^{-1}$.

Methyl 2-(4-cyanophenoxy)-2-(pyridin-3-yl)acetate (12q)<smiles>CC(=O)OC(Oc1ccc(C#N)cc1)c1cccnc1</smiles>

Compound 12q was prepared according to the general procedure GP-3 and was obtained after column chromatography ( $n$-pentane : $\mathrm{Et}_{2} \mathrm{O}$ 9:1 $\rightarrow$ 1:1); 41\% (22 mg); colorless oil.

${ }^{1}$ H NMR (400 MHz, Chloroform- $d$ ): $\delta=8.82-8.70(\mathrm{~m}, 1 \mathrm{H}), 8.60(\mathrm{~d}, J=4.7 \mathrm{~Hz}, 1 \mathrm{H}), 7.84(\mathrm{dt}, J=8.0$, $2.0 \mathrm{~Hz}, 1 \mathrm{H}), 7.65-7.47(\mathrm{~m}, 2 \mathrm{H}), 7.31(\mathrm{dd}, J=8.0,4.8 \mathrm{~Hz}, 1 \mathrm{H}), 6.98-6.90(\mathrm{~m}, 2 \mathrm{H}), 5.66(\mathrm{~s}, 1 \mathrm{H}), 3.71$ (s, 3H) ppm.

${ }^{13}$ C NMR (101 MHz, Chloroform- $d$ ): $\delta=168.7,160.0,150.7,148.6,134.5,134.2,123.8,118.6,115.9$, $105.8,76.3,53.1 \mathrm{ppm}$.

HRMS (ESI): $m / z$ : $[\mathrm{M}+\mathrm{H}]^{+}$Calcd. for $\mathrm{C}_{15} \mathrm{H}_{13} \mathrm{O}_{3} \mathrm{~N}: 269.0920$; Found: 269.0910 .

IR (KBr): 3834, 3407, 3201, 3054, 2954, 2859, 2332, 2224, 2094, 1991, 1908, 1752, 1601, 1503, 1431, $1333,1292,1243,1175,1121,1063,973,893,835,766,709 \mathrm{~cm}^{-1}$.

Methyl 2-phenoxy-2-(thiophen-2-yl)acetate (12r)<smiles>CC(=O)C(Oc1ccccc1)c1cccs1</smiles>

Compound 12r was prepared according to the general procedure GP-3 and was obtained after column chromatography ( $n$-pentane : $\left.\mathrm{Et}_{2} \mathrm{O} 20: 1 \rightarrow 4: 1\right) ; 91 \%(47 \mathrm{mg})$; yellow oil.

${ }^{1}$ H NMR (400 MHz, Chloroform- $d$ ): $\delta=7.64-7.53(\mathrm{~m}, 2 \mathrm{H}), 7.37(\mathrm{dd}, J=5.1,1.2 \mathrm{~Hz}, 1 \mathrm{H}), 7.22(\mathrm{dt}, J$ $=3.7,1.0 \mathrm{~Hz}, 1 \mathrm{H}), 7.09-6.91(\mathrm{~m}, 3 \mathrm{H}), 5.92(\mathrm{~s}, 1 \mathrm{H}), 3.79(\mathrm{~s}, 3 \mathrm{H}) \mathrm{ppm}$.

${ }^{13}$ C NMR (101 MHz, Chloroform- $d$ ): $\delta=168.5,160.0,135.9,134.1,127.5,127.4,127.0,118.7,116.0$, 105.6, 74.5, $53.1 \mathrm{ppm}$.

HRMS (ESI): $m / z$ : [M + Na $]^{+}$Calcd. for $\mathrm{C}_{14} \mathrm{H}_{11} \mathrm{O}_{3} \mathrm{NSNa}$ : 296.0351; Found: 296.0344 .

IR (KBr): 3489, 3104, 3006, 2954, 2847, 2546, 2226, 2159, 1973, 1905, 1745, 1601, 1433, 1325, 1294 , $1235,1172,1041,910,834,710 \mathrm{~cm}^{-1}$. 


\section{4-(2,2,2-Trifluoro-1-phenylethoxy)benzonitrile (13)}<smiles>N#Cc1ccc(OC(c2ccccc2)C(F)(F)F)cc1</smiles>

Compound 13 was prepared according to the general procedure GP-3 and was obtained after column chromatography ( $n$-pentane $\left.: \mathrm{Et}_{2} \mathrm{O} 20: 1 \rightarrow 9: 1\right) ; 43 \%$ (24 mg); colorless oil.

${ }^{1}$ H NMR (600 MHz, Chloroform- $\left.d\right): \delta=7.59-7.42(\mathrm{~m}, 7 \mathrm{H}), 7.04-6.88(\mathrm{~m}, 2 \mathrm{H}), 5.47$ (q, $J=6.1 \mathrm{~Hz}$, 1H) $\mathrm{ppm}$.

${ }^{13}$ C NMR (151 MHz, Chloroform- $\left.d\right): \delta=159.6,134.1,130.8,130.2,129.1,127.7,122.9$ (q, $J=281.1$ $\mathrm{Hz}), 118.5,116.6,105.9,78.1(\mathrm{q}, J=32.7 \mathrm{~Hz}) \mathrm{ppm}$.

${ }^{19}$ F NMR (565 MHz, Chloroform- $\left.d\right): \delta=-76.66(\mathrm{~d}, J=6.0 \mathrm{~Hz}) \mathrm{ppm}$.

HRMS (ESI): $m / z$ : $[\mathrm{M}]^{+}$Calcd. for $\mathrm{C}_{15} \mathrm{H}_{11} \mathrm{~F}_{3} \mathrm{NO}: 278.0787$; Found: 278.0774 .

IR (KBr): 3432, 3070, 2924, 2673, 2338, 2228, 2101, 1992, 1906, 1604, 1503, 1456, 1417, 1367, 1243 , $1174,1134,1052,894,834,757,701 \mathrm{~cm}^{-1}$.

\section{Methyl 2-(4-nitrophenoxy)-2-phenylacetate (15a)}<smiles>CC(=O)C(Oc1ccc([N+](=O)[O-])cc1)c1ccccc1</smiles>

Compound 15a was prepared according to the general procedure GP-3 and was obtained after column chromatography ( $n$-hexane : EtOAc 20:1 $\rightarrow$ 9:1); 85\% (49 mg); colorless oil.

${ }^{1}$ H NMR (400 MHz, Chloroform- $\left.d\right)$ : $\delta=8.29-8.14(\mathrm{~m}, 2 \mathrm{H}), 7.65-7.53(\mathrm{~m}, 2 \mathrm{H}), 7.54-7.37(\mathrm{~m}, 3 \mathrm{H})$, $7.11-6.98(\mathrm{~m}, 2 \mathrm{H}), 5.75(\mathrm{~s}, 1 \mathrm{H}), 3.79(\mathrm{~s}, 3 \mathrm{H}) \mathrm{ppm}$.

${ }^{13}$ C NMR (101MHz, Chloroform- $d$ ): $\delta=169.2,161.9,142.2,134.1,129.5,129.0,127.0,125.9,115.3$, $78.8,52.9 \mathrm{ppm}$.

HRMS (ESI): $m / z:[\mathrm{M}+\mathrm{Na}]^{+}$Calcd. for $\mathrm{C}_{15} \mathrm{H}_{13} \mathrm{O}_{5} \mathrm{NNa}$ : 310.0685; Found: 310.0686 .

IR (neat): 3082, 3034, 2953, 2847, 2652, 2451, 2339, 2255, 2213, 2110, 2089, 1903, 1751, 1590, 1511 , $1492,1435,1340,1298,1244,1169,1210,1110,1051,1083,1007,937,908,842,779,727,692$, $662 \mathrm{~cm}^{-1}$

\section{Methyl 2-phenoxy-2-phenylacetate (15b)}

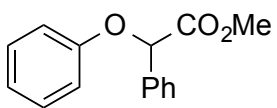

Compound 15b was prepared according to the general procedure GP-3 and was obtained after column chromatography ( $n$-hexane : EtOAc 20:1 $\rightarrow$ 9:1); 29\% (14 mg); colorless oil.

${ }^{1}$ H NMR (400 MHz, Chloroform- $\left.d\right): \delta=7.62-7.53(\mathrm{~m}, 2 \mathrm{H}), 7.45-7.34(\mathrm{~m}, 3 \mathrm{H}), 7.31-7.22(\mathrm{~m}, 2 \mathrm{H})$, $7.12-6.75(\mathrm{~m}, 3 \mathrm{H}), 5.64(\mathrm{~s}, 1 \mathrm{H}), 3.73(\mathrm{~s}, 3 \mathrm{H}) \mathrm{ppm}$.

${ }^{13}$ C NMR (101MHz, Chloroform- $d$ ): $\delta=170.4,157.2,135.3,129.5,128.9,128.7,127.0,121.8,115.4$, 78.5, $52.6 \mathrm{ppm}$.

The data is in accordance with the literature. ${ }^{[2]}$ 


\section{Methyl 2-(4-fluorophenoxy)-2-phenylacetate (15c)}<smiles>CC(=O)C(Oc1ccc(F)cc1)c1ccccc1</smiles>

Compound 15c was prepared according to the general procedure GP-3 and was obtained after column chromatography ( $n$-pentane : $\left.\mathrm{Et}_{2} \mathrm{O} 20: 1 \rightarrow 9: 1\right) ; 50 \%(26 \mathrm{mg})$; colorless oil.

${ }^{1}$ H NMR (600 MHz, Chloroform- $d$ ): $\delta=7.60-7.55(\mathrm{~m}, 2 \mathrm{H}), 7.46-7.36(\mathrm{~m}, 3 \mathrm{H}), 7.04-6.95(\mathrm{~m}, 2 \mathrm{H})$, $6.94-6.88(\mathrm{~m}, 2 \mathrm{H}), 5.59(\mathrm{~s}, 1 \mathrm{H}), 3.76(\mathrm{~s}, 3 \mathrm{H}) \mathrm{ppm}$.

${ }^{13}$ C NMR (151 MHz, Chloroform- $d$ ): $\delta=170.2,157.8(\mathrm{~d}, J=240.7 \mathrm{~Hz}), 153.3(\mathrm{~d}, J=1.8 \mathrm{~Hz}), 135.2$, $129.1,128.8,127.0,116.9(\mathrm{~d}, J=7.5 \mathrm{~Hz}), 116.0(\mathrm{~d}, J=24.1 \mathrm{~Hz}), 79.4,52.6 \mathrm{ppm}$.

${ }^{19}$ F NMR (565 MHz, Chloroform- $d$ ): $\delta=-122.2--122.3$ (m) ppm.

HRMS (ESI): $m / z:[\mathrm{M}+\mathrm{Na}]^{+}$Calcd. for $\mathrm{C}_{15} \mathrm{H}_{13} \mathrm{FNaO}_{3}: 283.0740$; Found: 283.0738 .

IR (KBr): 3489, 3066, 2954, 2326, 2167, 2115, 2019, 1889, 1752, 1600, 1501, 1451, 1330, 1270, 1201, $1091,1057,1009,920,828,771,728,696 \mathrm{~cm}^{-1}$.

\section{Methyl 2-(4-chlorophenoxy)-2-phenylacetate (15d)}

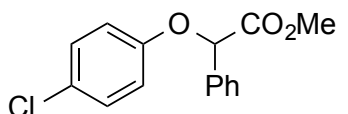

Compound 15d was prepared according to the general procedure GP-3 and was obtained after column chromatography ( $n$-pentane : $\left.\mathrm{Et}_{2} \mathrm{O} 20: 1 \rightarrow 9: 1\right) ; 38 \%(21 \mathrm{mg})$; colorless oil.

${ }^{1}$ H NMR (600 MHz, Chloroform- $d$ ): $\delta=7.62-7.55(\mathrm{~m}, 2 \mathrm{H}), 7.46-7.36(\mathrm{~m}, 3 \mathrm{H}), 7.26-7.20(\mathrm{~m}, 2 \mathrm{H})$, $6.96-6.83(\mathrm{~m}, 2 \mathrm{H}), 5.61(\mathrm{~s}, 1 \mathrm{H}), 3.76(\mathrm{~s}, 3 \mathrm{H}) \mathrm{ppm}$.

${ }^{13}$ C NMR (151 MHz, Chloroform- $\left.d\right): \delta=170.0,155.8,135.0,129.5,129.1,128.8,127.0,126.8,116.8$, $78.9,52.7 \mathrm{ppm}$.

HRMS (ESI): $m / z$ : [M + Na] $]^{+}$Calcd. for $\mathrm{C}_{15} \mathrm{H}_{13} \mathrm{ClNaO}_{3}$ : 299.0445; Found: 299.0442 .

IR (KBr): 3495, 3067, 3036, 2953, 2850, 2323, 2163, 2019, 1882, 1752, 1588, 1488, 1452, 1328, 1277 , $1171,1085,1056,1007,823,731,676 \mathrm{~cm}^{-1}$.

\section{Methyl 2-phenyl-2-(4-(trifluoromethoxy)phenoxy)acetate (15e)}

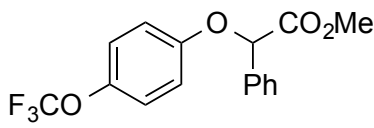

Compound 15e was prepared according to the general procedure GP-3 and was obtained after column chromatography ( $n$-pentane : $\left.\mathrm{Et}_{2} \mathrm{O} 20: 1 \rightarrow 9: 1\right) ; 60 \%$ (39 mg); colorless oil.

${ }^{1}$ H NMR (600 MHz, Chloroform- $d$ ): $\delta=7.62-7.55(\mathrm{~m}, 2 \mathrm{H}), 7.50-7.37(\mathrm{~m}, 3 \mathrm{H}), 7.16-7.11(\mathrm{~m}, 2 \mathrm{H})$, $7.03-6.94(\mathrm{~m}, 2 \mathrm{H}), 5.62(\mathrm{~s}, 1 \mathrm{H}), 3.77(\mathrm{~s}, 3 \mathrm{H}) \mathrm{ppm}$.

${ }^{13}$ C NMR (151 MHz, Chloroform- $d$ ): $\delta=170.0,155.7,143.5,134.9,129.2,128.9,127.0,122.4,120.5$ (q, $J=256.6 \mathrm{~Hz}), 116.4,79.0,52.7 \mathrm{ppm}$.

${ }^{19}$ F NMR $(565 \mathrm{MHz}$, Chloroform- $d$ ): $\delta=-58.33(\mathrm{~m}) \mathrm{ppm}$.

HRMS (ESI): $m / z$ : $[\mathrm{M}+\mathrm{Na}]^{+}$Calcd. for $\mathrm{C}_{16} \mathrm{H}_{13} \mathrm{O}_{4} \mathrm{~F}_{3} \mathrm{Na}$ : 349.0658; Found: 349.0655 .

IR (KBr): 3389, 3036, 2956, 2666, 2332, 2088, 1993, 1892, 1754, 1599, 1503, 1439, 1194, 1108, 1057, $1011,921,835,793,728,696 \mathrm{~cm}^{-1}$. 


\section{Methyl 2-phenyl-2-(4-(trifluoromethyl)phenoxy)acetate (15f)}<smiles>CC(=O)C(Oc1ccc(C(F)(F)F)cc1)c1ccccc1</smiles>

Compound $\mathbf{1 5 f}$ was prepared according to the general procedure GP-3 and was obtained after column chromatography ( $n$-hexane : EtOAc 20:1 $\rightarrow$ 9:1); 53\% (33 mg); colorless oil.

${ }^{1}$ H NMR (600 MHz, Chloroform- $d$ ): $\delta=7.66-7.58(\mathrm{~m}, 2 \mathrm{H}), 7.56(\mathrm{~d}, J=8.6 \mathrm{~Hz}, 2 \mathrm{H}), 7.51-7.36$ (m, $3 \mathrm{H}), 7.04(\mathrm{~d}, J=8.6 \mathrm{~Hz}, 2 \mathrm{H}), 5.71(\mathrm{~s}, 1 \mathrm{H}), 3.78(\mathrm{~s}, 3 \mathrm{H}) \mathrm{ppm}$.

${ }^{13}$ C NMR (151 MHz, Chloroform- $d$ ): $\delta=169.7,159.6,134.6,129.3,128.9,127.08,127.07$, (q, $J=3.7$ $\mathrm{Hz}) 124.2(\mathrm{q}, J=271.3 \mathrm{~Hz}), 124.0$ (q, $J=32.8 \mathrm{~Hz}), 115.4,78.5,52.8 \mathrm{ppm}$.

${ }^{19}$ F NMR $(565 \mathrm{MHz}$, Chloroform- $d$ ): $\delta=-61.71 \mathrm{ppm}$.

HRMS (ESI): $m / z$ : $[\mathrm{M}+\mathrm{Na}]^{+}$Calcd. for $\mathrm{C}_{16} \mathrm{H}_{13} \mathrm{O}_{3} \mathrm{~F}_{3} \mathrm{Na}$ : 333.0709; Found: 333.0709 .

IR (neat): 3486, 3077, 2955, 2930, 2849, 2297, 2214, 2089, 1906, 1815, 1750, 1612, 1515, 1436, 1324, $1246,1213,1152,1109,1050,1008,917,834,788,760,730,696 \mathrm{~cm}^{-1}$.

\section{Methyl 4-(2-methoxy-2-oxo-1-phenylethoxy)benzoate (15g)}<smiles>CC(=O)c1ccc(OC(C(C)=O)c2ccccc2)cc1</smiles>

Compound 15g was prepared according to the general procedure GP-3 and was obtained after column chromatography ( $n$-hexane : EtOAc 20:1 $\rightarrow$ 9:1 $\rightarrow$ 4:1); 73\% (44 mg); colorless oil.

${ }^{1}$ H NMR (600 MHz, Chloroform- $d$ ): $\delta=8.09-7.93(\mathrm{~m}, 2 \mathrm{H}), 7.63-7.54(\mathrm{~m}, 2 \mathrm{H}), 7.49-7.37(\mathrm{~m}, 3 \mathrm{H})$, $7.04-6.94(\mathrm{~m}, 2 \mathrm{H}), 5.73(\mathrm{~s}, 1 \mathrm{H}), 3.90(\mathrm{~s}, 3 \mathrm{H}), 3.77(\mathrm{~s}, 3 \mathrm{H}) \mathrm{ppm}$.

${ }^{13}$ C NMR (151 MHz, Chloroform- $d$ ): $\delta=169.8,166.6,160.8,134.7,131.6,129.2,128.9,127.0,123.7$, $114.9,78.4,52.7,51.9 \mathrm{ppm}$.

HRMS (ESI): $m / z$ : [M] $]^{+}$Calcd. for $\mathrm{C}_{17} \mathrm{H}_{16} \mathrm{O}_{5} \mathrm{H}: 301.1070$; Found: 301.1073.

IR: 3500, 3409, 3075, 3002, 2947, 2838, 2083, 1916, 1822, 1755, 1709, 1602, 1508, 1431, 1314, 1277 , $1240,1204,1163,1104,1062,987,961,913,854,820,770,729,692 \mathrm{~cm}^{-1}$.

\section{Methyl 2-(3-nitrophenoxy)-2-phenylacetate (15h)}<smiles>CC(=O)C(Oc1cccc([N+](=O)[O-])c1)c1ccccc1</smiles>

Compound 15h was prepared according to the general procedure GP-3 and was obtained after column chromatography ( $n$-hexane : EtOAc 20:1 $\rightarrow$ 9:1 $\rightarrow$ 4:1); 85\% (49 mg); colorless oil.

${ }^{1}$ H NMR (600 MHz, Chloroform- $d$ ): $\delta=7.90-7.87(\mathrm{~m}, 1 \mathrm{H}), 7.80(\mathrm{t}, J=2.3 \mathrm{~Hz}, 1 \mathrm{H}), 7.63-7.57$ (m, $2 \mathrm{H}), 7.50-7.39(\mathrm{~m}, 4 \mathrm{H}), 7.32$ (ddd, $J=8.3,2.6,0.9 \mathrm{~Hz}, 1 \mathrm{H}), 5.74(\mathrm{~s}, 1 \mathrm{H}), 3.79(\mathrm{~s}, 3 \mathrm{H}) \mathrm{ppm}$.

${ }^{13}$ C NMR (151 MHz, Chloroform- $d$ ): $\delta=169.4,157.6,134.2,130.2,129.4,129.0,127.1,122.2,116.8$, $110.1,78.8,52.9 \mathrm{ppm}$.

HRMS (ESI): $m / z$ : $[\mathrm{M}+\mathrm{Na}]^{+}$Calcd. for $\mathrm{C}_{15} \mathrm{H}_{13} \mathrm{O}_{5} \mathrm{NNa}$ : 310.0685; Found: 310.0686 .

IR (neat): 3407, 3093, 3033, 2953, 2340, 2100, 1750, 1614, 1582, 1524, 1477, 1453, 1348, 1285, 1209, $1172,1082,1054,1003,934,858,816,734,695 \mathrm{~cm}^{-1}$. 
<smiles>COC(=O)C(Oc1cccc(F)c1)c1ccccc1</smiles>

Compound 15i was prepared according to the general procedure GP-3 and was obtained after column chromatography ( $n$-hexane : EtOAc 20:1 $\rightarrow$ 9:1); 47\% (25 mg); colorless oil.

${ }^{1}$ H NMR (600 MHz, Chloroform- $d$ ): $\delta=7.61-7.56(\mathrm{~m}, 2 \mathrm{H}), 7.46-7.37(\mathrm{~m}, 3 \mathrm{H}), 7.27-7.20(\mathrm{~m}, 1 \mathrm{H})$, $6.79-6.73(\mathrm{~m}, 1 \mathrm{H}), 6.72-6.68(\mathrm{~m}, 2 \mathrm{H}), 5.64(\mathrm{~s}, 1 \mathrm{H}), 3.77(\mathrm{~s}, 3 \mathrm{H}) \mathrm{ppm}$.

${ }^{13}$ C NMR (151 MHz, Chloroform- $d$ ): $\delta=169.9,163.4(\mathrm{~d}, J=245.9 \mathrm{~Hz}), 158.4$ (d, $\left.J=11.0 \mathrm{~Hz}\right), 134.9$, $130.3(\mathrm{~d}, J=10.0 \mathrm{~Hz}), 129.1,128.8,127.0,111.0(\mathrm{~d}, J=2.9 \mathrm{~Hz}), 108.7$ (d, $J=21.2 \mathrm{~Hz}), 103.4(\mathrm{~d}, J=$ $25.3 \mathrm{~Hz}), 78.7,52.7 \mathrm{ppm}$.

${ }^{19}$ F NMR (565 MHz, Chloroform- $d$ ): $\delta=-111.14 \mathrm{ppm}$.

HRMS (ESI): $m / z$ : [M + Na] $]^{+}$Calcd. for $\mathrm{C}_{15} \mathrm{H}_{13} \mathrm{O}_{3} \mathrm{FNa}$ : 283.0740; Found: 283.0741.

IR (neat): 3033, 2953, 2246, 2126, 1813, 1753, 1593, 1486, 1451, 1259, 1209, 1164, 1133, 1054, 1003, $848,761,726,695 \mathrm{~cm}^{-1}$.

\section{Methyl 2-phenyl-2-(3-(trifluoromethyl)phenoxy)acetate (15j)}<smiles>CC(=O)C(Oc1cccc(C(F)(F)F)c1)c1ccccc1</smiles>

Compound 15j was prepared according to the general procedure GP-3 and was obtained after column chromatography ( $n$-hexane : EtOAc 20:1 $\rightarrow$ 9:1); 42\% (26 mg); colorless oil.

${ }^{1}$ H NMR (600 MHz, Chloroform- $d$ ): $\delta=7.54-7.47(\mathrm{~m}, 2 \mathrm{H}), 7.38-7.27(\mathrm{~m}, 4 \mathrm{H}), 7.19-7.15(\mathrm{~m}, 1 \mathrm{H})$, $7.16-7.12(\mathrm{~m}, 1 \mathrm{H}), 7.02(\mathrm{dd}, J=8.4,2.6 \mathrm{~Hz}, 1 \mathrm{H}), 5.59(\mathrm{~s}, 1 \mathrm{H}), 3.68(\mathrm{~s}, 3 \mathrm{H})$.

${ }^{13}$ C NMR (151 MHz, Chloroform- $d$ ): $\delta=169.8,157.3,134.7,132.0$ (q, $\left.J=32.4 \mathrm{~Hz}\right), 130.1,129.2$, $128.9,127.1,123.7$ (q, $J=272.5 \mathrm{~Hz}), 118.5$ (q, $J=3.8 \mathrm{~Hz}), 118.5,112.8$ (q, $J=3.8 \mathrm{~Hz}), 78.7,52.7 \mathrm{ppm}$. ${ }^{19}$ F NMR (565 MHz, Chloroform- $d$ ): $\delta=-62.82 \mathrm{ppm}$.

HRMS (ESI): $m / z$ : [M + Na] $]^{+}$Calcd. for $\mathrm{C}_{16} \mathrm{H}_{13} \mathrm{~F}_{3} \mathrm{O}_{3} \mathrm{Na}$ : 333.0709; Found: 333.0708 .

IR (neat): 3068, 3036, 2956, 2850, 2653, 2126, 1954, 1821, 1755, 1691, 1592, 1493, 1451, 1325, 1293, $1209,1165,1121,1097,1058,1013,979,936,882,784,755,729,694,669 \mathrm{~cm}^{-1}$.

\section{Methyl 2-(2-cyanophenoxy)-2-phenylacetate (15k)}<smiles>CC(=O)C(Oc1ccccc1N)c1ccccn1</smiles>

Compound 15k was prepared according to the general procedure GP-3 and was obtained after column chromatography ( $n$-hexane : EtOAc 20:1 $\rightarrow$ 9:1); 41\% (22 mg); colorless oil.

${ }^{1}$ H NMR $(600 \mathrm{MHz}$, Chloroform- $d): \delta=7.70-7.66(\mathrm{~m}, 2 \mathrm{H}), 7.64(\mathrm{dd}, J=7.7,1.7 \mathrm{~Hz}, 1 \mathrm{H}), 7.54-7.50$ (m, 1H), $7.48-7.39$ (m, 3H), 7.09 (td, $J=7.6,0.9 \mathrm{~Hz}, 1 \mathrm{H}), 6.92$ (d, $J=8.5 \mathrm{~Hz}, 1 \mathrm{H}), 5.76(\mathrm{~s}, 1 \mathrm{H}), 3.76$ (s, 3H) ppm.

${ }^{13}$ C NMR (151 MHz, Chloroform- $d$ ): $\delta=169.3,158.8,134.2,134.1,134.0,129.3,128.9,126.9,121.9$, 116.0, 113.1, 103.2, 79.0, $52.9 \mathrm{ppm}$.

HRMS (ESI): $m / z$ : $[\mathrm{M}+\mathrm{Na}]^{+}$Calcd. for $\mathrm{C}_{16} \mathrm{H}_{13} \mathrm{O}_{3} \mathrm{NNa}$ : 290.0787; Found: 290.0788 .

IR (neat): 3012, 2958, 2922, 2851, 2342, 2227, 2098, 1815, 1732, 1596, 1491, 1449, 1356, 1318, 1288, $1256,1212,1168,1113,1058,974,841,764,724,691 \mathrm{~cm}^{-1}$. 
Methyl 2-(2-nitrophenoxy)-2-phenylacetate (151)<smiles>COC(=O)C(Oc1ccccc1[N+](=O)[O-])c1ccccc1</smiles>

Compound 15l was prepared according to the general procedure GP-3 and was obtained after column chromatography ( $n$-hexane : EtOAc 20:1 $\rightarrow$ 9:1); 37\% (21 mg); colorless oil.

${ }^{1}$ H NMR (400 MHz, Chloroform- $d$ ): $\delta=7.92(\mathrm{dd}, J=8.1,1.7 \mathrm{~Hz}, 1 \mathrm{H}), 7.70-7.59(\mathrm{~m}, 2 \mathrm{H}), 7.57-7.34$ $(\mathrm{m}, 4 \mathrm{H}), 7.15-7.10(\mathrm{~m}, 1 \mathrm{H}), 7.02(\mathrm{dd}, J=8.4,1.1 \mathrm{~Hz}, 1 \mathrm{H}), 5.79(\mathrm{~s}, 1 \mathrm{H}), 3.75(\mathrm{~s}, 3 \mathrm{H}) \mathrm{ppm}$.

${ }^{13}$ C NMR (101 MHz, Chloroform- $d$ ): $\delta=169.2,150.5,134.08,134.01,129.3,128.9,127.0,126.0$, $121.8,115.7,79.7,52.9 \mathrm{ppm}$.

HRMS (ESI): $m / z$ : $[\mathrm{M}+\mathrm{Na}]^{+}$Calcd. for $\mathrm{C}_{15} \mathrm{H}_{13} \mathrm{O}_{5} \mathrm{NNa}$ : 310.0685 ; Found: 310.0685 .

IR (neat): 3068, 3034, 2953, 2852, 2399, 2101, 1750, 1603, 1521, 1484, 1452, 1349, 1276, 1246, 1209 , $1167,1092,1048,973,911,859,823,771,741,694,670 \mathrm{~cm}^{-1}$.

Methyl 2-(2-chlorophenoxy)-2-phenylacetate (15m)

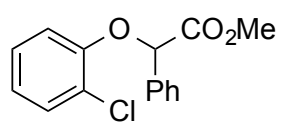

Compound $\mathbf{1 5 m}$ was prepared according to the general procedure GP-3 and was obtained after column chromatography ( $n$-hexane : EtOAc 20:1 $\rightarrow$ 9:1); 20\% (10 mg); colorless oil.

${ }^{1}$ H NMR $(600 \mathrm{MHz}$, Chloroform- $d): \delta=7.69-7.62(\mathrm{~m}, 2 \mathrm{H}), 7.49-7.35(\mathrm{~m}, 4 \mathrm{H}), 7.19-7.15(\mathrm{~m}, 1 \mathrm{H})$, $6.97(\mathrm{td}, J=7.7,1.4 \mathrm{~Hz}, 1 \mathrm{H}), 6.88(\mathrm{dd}, J=8.2,1.4 \mathrm{~Hz}, 1 \mathrm{H}), 5.70(\mathrm{~s}, 1 \mathrm{H}), 3.76(\mathrm{~s}, 3 \mathrm{H}) \mathrm{ppm}$.

${ }^{13}$ C NMR (151 MHz, Chloroform- $d$ ): $\delta=169.9,152.9,134.9,130.6,129.0,128.7,127.60,127.0,124.1$, $122.8,115.2,79.4,52.7 \mathrm{ppm}$.

HRMS (ESI): $m / z$ : $[\mathrm{M}+\mathrm{Na}]^{+}$Calcd. for $\mathrm{C}_{15} \mathrm{H}_{13} \mathrm{O}_{3} \mathrm{ClNa}$ 299.0445; Found: 299.0442.

IR (neat): 3009, 2955, 2922, 2850, 2321, 2227, 2115, 1937, 1813, 1733, 1594, 1486, 1448, 1355, 1317, $1278,1254,1210,1167,1113,1057,974,838,758,722,690 \mathrm{~cm}^{-1}$.

Methyl 2-(naphthalen-1-yloxy)-2-phenylacetate (15n)<smiles>CC(=O)C(Oc1cccc2ccccc12)c1ccccc1</smiles>

Compound 15n was prepared according to the general procedure GP-3 and was obtained after column chromatography (n-pentane : $\left.\mathrm{Et}_{2} \mathrm{O} 20: 1 \rightarrow 9: 1\right) ; 27 \%$ (16 mg); colorless oil.

${ }^{1}$ H NMR $(600 \mathrm{MHz}$, Chloroform- $d$ ): $\delta=8.56-8.40(\mathrm{~m}, 1 \mathrm{H}), 7.84-7.80(\mathrm{~m}, 1 \mathrm{H}), 7.76-7.69(\mathrm{~m}, 2 \mathrm{H})$, $7.57-7.51(\mathrm{~m}, 2 \mathrm{H}), 7.50-7.41(\mathrm{~m}, 4 \mathrm{H}), 7.33(\mathrm{t}, J=8.0 \mathrm{~Hz}, 1 \mathrm{H}), 6.76(\mathrm{~d}, J=7.6 \mathrm{~Hz}, 1 \mathrm{H}), 5.86(\mathrm{~s}, 1 \mathrm{H})$, 3.77 (s, 3H) ppm.

${ }^{13}$ C NMR (151 MHz, Chloroform- $d$ ): $\delta=170.4,153.0,135.5,134.6,129.2,129.0,128.8,127.4,127.0$, $126.6,125.8,125.5,122.3,121.4,106.0,78.8,52.6 \mathrm{ppm}$.

HRMS (ESI): $m / z$ : [M + Na $]^{+}$Calcd. for $\mathrm{C}_{19} \mathrm{H}_{16} \mathrm{NaO}_{3}: 315.0991$; Found: 315.0986 .

IR (KBr): 3849, 3499, 3058, 2952, 2848, 2528, 2324, 2222, 2104, 2022, 1932, 1811, 1753, 1629, 1579, $1504,1456,1397,1346,1264,1239,1207,1169,1105,1068,1021,973,891,852,770,727,696 \mathrm{~cm}^{-1}$. 
Methyl 2-phenyl-2-(pyridin-2-yloxy)acetate (150)

$\overbrace{\mathrm{N}}^{\mathrm{O}} \mathrm{Y}_{\mathrm{Ph}}^{\mathrm{CO}_{2} \mathrm{Me}}$

Compound 150 was prepared according to the general procedure GP-3 and was obtained after column chromatography ( $n$-hexane : EtOAc 20:1 $\rightarrow$ 9:1 $\rightarrow$ 4:1); 76\% (37 mg); colorless oil.

${ }^{1}$ H NMR (400 MHz, Chloroform- $d$ ): $\delta=8.19-8.11(\mathrm{~m}, 1 \mathrm{H}), 7.70-7.58(\mathrm{~m}, 3 \mathrm{H}), 7.47-7.37(\mathrm{~m}, 3 \mathrm{H})$, $7.01-6.88(\mathrm{~m}, 2 \mathrm{H}), 6.26(\mathrm{~s}, 1 \mathrm{H}), 3.75(\mathrm{~s}, 3 \mathrm{H}) \mathrm{ppm}$.

${ }^{13}$ C NMR (101 MHz, Chloroform- $d$ ): $\delta=170.8,162.3,146.5,138.9,135.1,128.9,128.7,127.6,117.6$, $111.3,75.6,52.4 \mathrm{ppm}$.

HRMS (ESI): $\mathrm{m} / \mathrm{z}$ : [M + Na] $]^{+}$Calcd. for $\mathrm{C}_{14} \mathrm{H}_{13} \mathrm{O}_{3} \mathrm{NNa}$ : 266.0787; Found: 266.0788 .

IR (neat): 3030, 2951, 2550, 2120, 1955, 1751, 1594, 1468, 1430, 1348, 1245, 1208, 1170, 1055, 1028, $986,892,847,776,728,694 \mathrm{~cm}^{-1}$.

\section{Methyl 2-phenyl-2-(quinolin-2-yloxy)acetate (15p)}<smiles>COC(=O)c1ccccc1Oc1ccc2ccccc2n1</smiles>

Compound 15p was prepared according to the general procedure GP-3 and was obtained after column chromatography (n-pentane : $\left.\mathrm{Et}_{2} \mathrm{O} 20: 1 \rightarrow 9: 1\right) ; 70 \%$ (41 mg); colorless oil.

${ }^{1}$ H NMR $(600 \mathrm{MHz}$, Chloroform- $d): \delta=8.07(\mathrm{~d}, J=8.7 \mathrm{~Hz}, 1 \mathrm{H}), 7.88-7.82(\mathrm{~m}, 1 \mathrm{H}), 7.77-7.75(\mathrm{~m}$, $1 \mathrm{H}), 7.72-7.68(\mathrm{~m}, 2 \mathrm{H}), 7.657-7.63(\mathrm{~m}, 1 \mathrm{H}), 7.49-7.39(\mathrm{~m}, 4 \mathrm{H}), 7.12(\mathrm{~d}, J=8.8 \mathrm{~Hz}, 1 \mathrm{H}), 6.46$ (s, $1 \mathrm{H}), 3.77$ (s, 3H) ppm.

${ }^{13}$ C NMR (151 MHz, Chloroform- $d$ ): $\delta=170.8,160.5,146.0,139.2,135.0,129.6,129.0,128.7,127.8$, $127.46,127.43,125.4,124.4,112.7,75.8,52.4 \mathrm{ppm}$.

HRMS (ESI): $m / z$ : [M + Na] $]^{+}$Calcd. for $\mathrm{C}_{18} \mathrm{H}_{15} \mathrm{NNaO}_{3}: 316.0944$; Found: 316.0941.

IR (KBr): 3491, 3061, 2952, 2847, 2632, 2293, 2167, 2114, 1958, 1750, 1604, 1577, 1506, 1470, 1429, $2392,1345,1315,1327,1210,1172,1110,1082,990,921,872,822,755,725,695 \mathrm{~cm}^{-1}$. 


\section{Computational Calculations}

\section{Computational Details}

All the calculations were performed with the Gaussian 09 program. ${ }^{[3]}$ Geometry optimizations of diazo acetates 6a, phenols 10a-y and phenol-diazo complexes involved were carried out at the B3LYP method ${ }^{[4]}$ with $6-31 G(d)$ basis set $^{[5]}$ in the gas phase. Harmonic vibrational frequency calculations at the same level were performed for all of the stationary points to confirm them as a local minima or transition structures. Furthermore, to improve the calculation accuracy, single-point calculations were computed at the MP2 method ${ }^{[6]}$ with the $6-311+\mathrm{G}(\mathrm{d}, \mathrm{p})$ basis set. ${ }^{[7]}$ The basis set superposition error (BSSE) is estimated by employing the counterpoise method at the same calculation method. ${ }^{[8]}$ The interaction energies were calculated according to the formula: $E($ interaction $)=E($ complex $)-E($ diazo $)-E($ phenol $)+$ BSSE. Furthermore, we have also considered the solvent effects in chloroform $(\varepsilon=4.7113)$ using the SMD solvation model, ${ }^{[9]}$ with single-point calculations based on the gas-phase optimized geometries. The interaction energies in solution cannot be calculated accurately and only for reference, which calculated according to the formula: $E$ (interaction) $)_{\text {sol }}=$ $E(\text { complex })_{\text {sol }}-E(\text { diazo })_{\text {sol }}-E(\text { phenol })_{\text {sol }}+$ BSSE. The computed structures are plotted using CYLView. ${ }^{[10]}$

\section{Computed Energies of All Stationary Points}

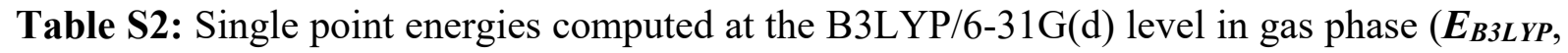
in Hartree), single point energies computed at the MP2/6-311+G(d,p) level in gas phase ( $\boldsymbol{E}_{\boldsymbol{M P} 2}$, in Hartree), the basis set superposition error (BSSE) computed at the MP2/6$311+\mathrm{G}(\mathrm{d}, \mathrm{p})$ level using the counterpoise method (BSSE, in Hartree), and single point energies in chloroform computed at the MP2/6-311+G(d,p) level $\left(\boldsymbol{E}_{\boldsymbol{s} o l}\right.$, in Hartree).

\begin{tabular}{|c|c|c|c|c|}
\hline Name & $E_{B 3 L Y P}$ & $E_{M P 2}$ & BSSE & $E_{\text {sol }}$ \\
\hline $6 \mathbf{6 a}$ & -607.682770 & -606.175522 & -- & -606.184475 \\
\hline $10 a$ & -307.464868 & -306.665662 & -- & -306.678070 \\
\hline $11 \mathrm{a}$ & -915.160062 & -912.859715 & 0.00568156 & -912.876981 \\
\hline $10 \mathrm{~b}$ & -399.710013 & -398.704378 & -- & -398.719671 \\
\hline $11 b$ & -1007.407603 & -1004.900793 & 0.00574073 & -1004.919896 \\
\hline $10 \mathrm{c}$ & -511.969013 & -510.772160 & -- & -510.786480 \\
\hline $11 \mathrm{c}$ & -1119.667041 & -1116.968761 & 0.00580840 & -1116.986950 \\
\hline 10d & -406.696393 & -405.741637 & -- & -405.754012 \\
\hline 11d & -1014.392216 & -1011.936332 & 0.00566954 & -1011.953098 \\
\hline
\end{tabular}




\begin{tabular}{|c|c|c|c|c|}
\hline $10 \mathrm{e}$ & -767.060325 & -765.724532 & -- & -765.738315 \\
\hline 11e & -1374.756636 & -1371.919839 & 0.00597464 & -1371.938006 \\
\hline $10 f$ & -644.503738 & -643.125557 & -- & -643.138301 \\
\hline $11 f$ & -1252.200527 & -1249.321499 & 0.00580664 & -1249.338306 \\
\hline $10 \mathrm{~g}$ & -421.984856 & -420.922240 & -- & -420.935554 \\
\hline $11 \mathrm{~g}$ & -1029.679919 & -1027.116332 & 0.00573394 & -1027.134601 \\
\hline 10h & -719.720230 & -718.196920 & -- & -718.208507 \\
\hline $11 \mathrm{~h}$ & -1327.416817 & -1324.392889 & 0.00590562 & -1324.408598 \\
\hline $10 \mathrm{i}$ & -535.346912 & -534.041460 & -- & -534.055646 \\
\hline $11 \mathbf{i}$ & -1143.043229 & -1140.236499 & 0.00566746 & -1140.255202 \\
\hline 10j & -346.782993 & -345.865878 & -- & -345.878921 \\
\hline $11 \mathrm{j}$ & -954.477985 & -952.060458 & 0.00594495 & -952.078225 \\
\hline 10k & -511.966831 & -510.772708 & -- & -510.786163 \\
\hline $11 k$ & -1119.666411 & -1116.969674 & 0.00528044 & -1116.985546 \\
\hline 101 & -406.698515 & -405.743583 & -- & -405.755745 \\
\hline 111 & -1014.394422 & -1011.938323 & 0.00564518 & -1011.955132 \\
\hline $10 \mathrm{~m}$ & -767.060821 & -765.725553 & -- & -765.739248 \\
\hline $11 \mathrm{~m}$ & -1374.757278 & -1371.921086 & 0.00625352 & -1371.939239 \\
\hline $10 n$ & -644.502790 & -643.125382 & -- & -643.137677 \\
\hline 11n & -1252.199028 & -1249.320836 & 0.00575385 & -1249.337538 \\
\hline 100 & -421.988095 & -420.924979 & -- & -420.938243 \\
\hline 110 & -1029.683057 & -1027.120668 & 0.00663870 & -1027.138830 \\
\hline $10 p$ & -535.345348 & -534.041722 & -- & -534.055277 \\
\hline $11 p$ & -1143.043940 & -1140.237233 & 0.00546955 & -1140.253479 \\
\hline $10 q$ & -346.783527 & -345.867385 & -- & -345.880180 \\
\hline $11 q$ & -954.478607 & -952.061578 & 0.00578486 & -952.079273 \\
\hline $10 \mathrm{r}$ & -399.711785 & -398.705915 & -- & -398.717629 \\
\hline $11 r$ & -1007.405262 & -1004.899227 & 0.00581611 & -1004.918538 \\
\hline 10u & -511.976067 & -510.775426 & -- & -510.784973 \\
\hline $11 u$ & -1119.664610 & -1116.962890 & 0.00475716 & -1116.978786 \\
\hline $10 v$ & -406.697592 & -405.742504 & -- & -405.752443 \\
\hline $11 v$ & -1014.392724 & -1011.934674 & 0.00683174 & -1011.950693 \\
\hline $10 w$ & -767.062655 & -765.727187 & -- & -765.737797 \\
\hline $11 w$ & -1374.754302 & -1371.919108 & 0.00649989 & -1371.937632 \\
\hline $10 x$ & -421.988917 & -420.927532 & -- & -420.938564 \\
\hline
\end{tabular}




\begin{tabular}{lllll}
$\mathbf{1 1 x}$ & -1029.680456 & -1027.120051 & 0.00784616 & -1027.138504 \\
$\mathbf{1 0 y}$ & -535.357338 & -534.048979 & - & -534.059231 \\
$\mathbf{1 1 y}$ & -1143.044906 & -1140.236625 & 0.00571098 & -1140.252992 \\
\hline
\end{tabular}

\section{Coordinates of All Stationary Points}

$\begin{array}{lrrr}\mathbf{6 a} & & & \\ & & & \\ \mathrm{C} & -2.75371500 & -1.53841500 & -0.00024800 \\ \mathrm{C} & -1.38838200 & -1.25571600 & 0.00007200 \\ \mathrm{C} & -0.94332600 & 0.07867200 & 0.00023400 \\ \mathrm{C} & -1.90435200 & 1.10724000 & 0.00003000 \\ \mathrm{C} & -3.26463700 & 0.81215200 & -0.00029600 \\ \mathrm{C} & -3.70016100 & -0.51394800 & -0.00043800 \\ \mathrm{H} & -3.07544000 & -2.57661800 & -0.00035400 \\ \mathrm{H} & -0.66370900 & -2.05869700 & 0.00022400 \\ \mathrm{H} & -1.59460900 & 2.14876700 & 0.00012300 \\ \mathrm{H} & -3.98505900 & 1.62578100 & -0.00044700 \\ \mathrm{H} & -4.76192200 & -0.74375300 & -0.00070000 \\ \mathrm{C} & 0.50018700 & 0.40143800 & 0.00063900 \\ \mathrm{C} & 1.62063800 & -0.55141800 & 0.00068400 \\ \mathrm{O} & 1.51685100 & -1.76310500 & 0.00015900 \\ \mathrm{O} & 2.81510500 & 0.09430600 & 0.00015200 \\ \mathrm{C} & 3.97075800 & -0.75862900 & -0.00046800 \\ \mathrm{H} & 4.82686600 & -0.08353500 & -0.00081200 \\ \mathrm{H} & 3.98126200 & -1.39287800 & 0.88973600 \\ \mathrm{H} & 3.98043200 & -1.39272600 & -0.89079100 \\ \mathrm{~N} & 0.84957900 & 1.67173800 & 0.00052500 \\ \mathrm{~N} & 1.12391700 & 2.77623300 & -0.00062800\end{array}$

$10 a$

$\begin{array}{lrrr}\mathrm{C} & 1.17171000 & -1.19034100 & -0.00000800 \\ \mathrm{C} & -0.22073300 & -1.22421800 & 0.00003700 \\ \mathrm{C} & -0.94102300 & -0.02457400 & 0.00002700 \\ \mathrm{C} & -0.26396200 & 1.19953400 & -0.00002000 \\ \mathrm{C} & 1.13169000 & 1.21933600 & 0.00000400 \\ \mathrm{C} & 1.85742800 & 0.02790700 & -0.00000200 \\ \mathrm{H} & 1.72573300 & -2.12548900 & -0.00000200 \\ \mathrm{H} & -0.76579600 & -2.16300000 & 0.00010900 \\ \mathrm{H} & -0.82681100 & 2.13177000 & -0.00011100 \\ \mathrm{H} & 1.64979700 & 2.17480400 & 0.00000600 \\ \mathrm{H} & 2.94324900 & 0.04714800 & -0.00000400\end{array}$




$\begin{array}{llrc}\mathrm{O} & -2.30724800 & -0.11186500 & -0.00013500 \\ \mathrm{H} & -2.67884200 & 0.78382900 & 0.00085100\end{array}$

11a

$\begin{array}{lrrr}\mathrm{C} & 0.03377200 & 2.63540800 & -0.54467200 \\ \mathrm{C} & 0.56577300 & 1.34698100 & -0.52381400 \\ \mathrm{C} & 1.87524200 & 1.13491700 & -0.06191500 \\ \mathrm{C} & 2.63615600 & 2.23894600 & 0.35876400 \\ \mathrm{C} & 2.10188100 & 3.52450900 & 0.31843800 \\ \mathrm{C} & 0.79530600 & 3.72832700 & -0.12865100 \\ \mathrm{H} & -0.98405400 & 2.77905000 & -0.89576400 \\ \mathrm{H} & -0.03553500 & 0.51529900 & -0.86706500 \\ \mathrm{H} & 3.64795500 & 2.09364200 & 0.72902900 \\ \mathrm{H} & 2.70594400 & 4.36537800 & 0.64816300 \\ \mathrm{H} & 0.37510100 & 4.72983500 & -0.15268300 \\ \mathrm{C} & 2.45955800 & -0.22597300 & -0.03377500 \\ \mathrm{C} & 1.73134500 & -1.47636000 & 0.16930200 \\ \mathrm{O} & 0.52428900 & -1.56028900 & 0.35136300 \\ \mathrm{O} & 2.54808200 & -2.55090400 & 0.14538000 \\ \mathrm{C} & 1.90945400 & -3.82529600 & 0.34250000 \\ \mathrm{H} & 2.71529900 & -4.55792000 & 0.30220800 \\ \mathrm{H} & 1.40789000 & -3.85656300 & 1.31274000 \\ \mathrm{H} & 1.17893000 & -4.01349000 & -0.44821600 \\ \mathrm{~N} & 3.76796100 & -0.34140900 & -0.15645500 \\ \mathrm{~N} & 4.89765900 & -0.41734300 & -0.26022800 \\ \mathrm{C} & -5.16810600 & 0.03181400 & -0.50469500 \\ \mathrm{C} & -4.08440500 & -0.54998600 & -1.15870300 \\ \mathrm{C} & -2.88530900 & -0.76230600 & -0.46648400 \\ \mathrm{C} & -2.78019100 & -0.38493600 & 0.87919200 \\ \mathrm{C} & -3.87486500 & 0.19557400 & 1.52135500 \\ \mathrm{C} & -5.07327300 & 0.40839600 & 0.83815900 \\ \mathrm{H} & -6.09546500 & 0.19105800 & -1.04953900 \\ \mathrm{H} & -4.14566200 & -0.84921100 & -2.20075900 \\ \mathrm{H} & -1.84525700 & -0.54427500 & 1.41002100 \\ \mathrm{H} & -3.78454600 & 0.48451900 & 2.56567500 \\ \mathrm{H} & -5.92180700 & 0.86043700 & 1.34373700 \\ \mathrm{H} & -1.85225800 & -1.33089400 & -1.15377400 \\ & -1.09308400 & -1.46989100 & -0.55451300\end{array}$

10b

$\begin{array}{lrrr}\mathrm{C} & -0.37720600 & 1.21679500 & -0.00001200 \\ \mathrm{C} & 1.00966100 & 1.22607200 & 0.00003600\end{array}$ 


$\begin{array}{lrrr}\mathrm{C} & 1.71480300 & 0.01469400 & 0.00005000 \\ \mathrm{C} & 1.02169800 & -1.20285400 & 0.00002100 \\ \mathrm{C} & -0.36822900 & -1.21014200 & -0.00001300 \\ \mathrm{C} & -1.08328100 & -0.00121100 & -0.00003500 \\ \mathrm{H} & -0.92645500 & 2.15268900 & -0.00002200 \\ \mathrm{H} & 1.56629800 & 2.15727900 & 0.00006800 \\ \mathrm{H} & 1.57007100 & -2.14252900 & 0.00001800 \\ \mathrm{H} & -0.90639100 & -2.15241300 & -0.00003100 \\ \mathrm{O} & 3.07348100 & 0.08717900 & 0.00006000 \\ \mathrm{H} & 3.44509000 & -0.80912000 & 0.00039700 \\ \mathrm{C} & -2.51482500 & -0.00882000 & -0.00007500 \\ \mathrm{~N} & -3.67888400 & -0.01579300 & -0.00010600\end{array}$

11b

$\begin{array}{lrrr}\mathrm{C} & -0.40798600 & 2.52832600 & 0.91810300 \\ \mathrm{C} & -1.05135700 & 1.30158400 & 0.76118200 \\ \mathrm{C} & -2.27650400 & 1.23176200 & 0.07776100 \\ \mathrm{C} & -2.84285200 & 2.41501000 & -0.42577700 \\ \mathrm{C} & -2.20081100 & 3.63854200 & -0.25027800 \\ \mathrm{C} & -0.97706500 & 3.70039600 & 0.41827100 \\ \mathrm{H} & 0.54287300 & 2.56328700 & 1.44212300 \\ \mathrm{H} & -0.60045000 & 0.40575400 & 1.16823700 \\ \mathrm{H} & -3.78541400 & 2.38017100 & -0.96633700 \\ \mathrm{H} & -2.65428000 & 4.54232100 & -0.64770000 \\ \mathrm{H} & -0.47184300 & 4.65314000 & 0.54818900 \\ \mathrm{C} & -2.97721000 & -0.06186000 & -0.09613300 \\ \mathrm{C} & -2.35140500 & -1.37277500 & -0.23676400 \\ \mathrm{O} & -1.14255200 & -1.57339200 & -0.23656800 \\ \mathrm{O} & -3.25794600 & -2.35969900 & -0.38216400 \\ \mathrm{C} & -2.72339900 & -3.68725100 & -0.53881500 \\ \mathrm{H} & -3.59353000 & -4.33337200 & -0.65204000 \\ \mathrm{H} & -2.08541100 & -3.74083300 & -1.42416600 \\ \mathrm{H} & -2.14476500 & -3.97279500 & 0.34311900 \\ \mathrm{~N} & -4.29439500 & -0.04658500 & -0.18249500 \\ \mathrm{~N} & -5.42767800 & -0.01008400 & -0.25825300 \\ \mathrm{C} & 4.54489700 & -0.52547100 & 0.95563600 \\ \mathrm{C} & 3.39542100 & -1.04676900 & 1.52995500 \\ \mathrm{C} & 2.20560900 & -1.11302500 & 0.78779600 \\ \mathrm{C} & 2.18096400 & -0.64723500 & -0.53719000 \\ \mathrm{C} & 3.33464300 & -0.12667200 & -1.10932300 \\ \mathrm{C} & 4.52976800 & -0.05839200 & -0.37238500 \\ \mathrm{H} & 5.46533800 & -0.47544000 & 1.52883200 \\ \mathrm{H} & 3.39419400 & -1.41184800 & 2.55190000\end{array}$




$\begin{array}{lrrr}\mathrm{H} & 1.25636800 & -0.69241800 & -1.10470300 \\ \mathrm{H} & 3.31672200 & 0.23387000 & -2.13307500 \\ \mathrm{O} & 1.11405800 & -1.62751500 & 1.40239100 \\ \mathrm{H} & 0.35759800 & -1.65770100 & 0.78065300 \\ \mathrm{C} & 5.71549000 & 0.48078400 & -0.96450200 \\ \mathrm{~N} & 6.67991600 & 0.92081000 & -1.44632700\end{array}$

10c

$\begin{array}{lrrr}\mathrm{C} & 0.01330700 & 1.22228000 & -0.00016700 \\ \mathrm{C} & -1.37312400 & 1.22917700 & -0.00013900 \\ \mathrm{C} & -2.07708900 & 0.01537800 & 0.00004700 \\ \mathrm{C} & -1.38402800 & -1.20413200 & 0.00020600 \\ \mathrm{C} & 0.00510900 & -1.21271000 & 0.00018100 \\ \mathrm{C} & 0.69133900 & 0.00073600 & -0.00000300 \\ \mathrm{H} & 0.58112100 & 2.14430600 & -0.00030300 \\ \mathrm{H} & -1.93112600 & 2.15924500 & -0.00025700 \\ \mathrm{H} & -1.93305900 & -2.14310200 & 0.00035200 \\ \mathrm{H} & 0.56357400 & -2.14039900 & 0.00030500 \\ \mathrm{O} & -3.43327100 & 0.08689800 & 0.00005500 \\ \mathrm{H} & -3.80565700 & -0.80929900 & 0.00027500 \\ \mathrm{~N} & 2.15367400 & -0.00698300 & -0.00002300 \\ \mathrm{O} & 2.73438800 & 1.08007300 & -0.00026300 \\ \mathrm{O} & 2.72342600 & -1.10025300 & 0.00008800\end{array}$

11c

$\begin{array}{lrrr}\mathrm{C} & 0.79182500 & 2.51961900 & -1.00906500 \\ \mathrm{C} & 1.43239400 & 1.29608500 & -0.81924800 \\ \mathrm{C} & 2.63234700 & 1.23520200 & -0.09167700 \\ \mathrm{C} & 3.17690500 & 2.42420900 & 0.42227500 \\ \mathrm{C} & 2.53820800 & 3.64432200 & 0.21424200 \\ \mathrm{C} & 1.33919100 & 3.69729600 & -0.49834900 \\ \mathrm{H} & -0.13935900 & 2.54790400 & -1.56772600 \\ \mathrm{H} & 0.99873500 & 0.39563600 & -1.23468000 \\ \mathrm{H} & 4.09933000 & 2.39674400 & 0.99686700 \\ \mathrm{H} & 2.97451200 & 4.55247700 & 0.62074100 \\ \mathrm{H} & 0.83628200 & 4.64740100 & -0.65385000 \\ \mathrm{C} & 3.32998100 & -0.05466300 & 0.11887000 \\ \mathrm{C} & 2.70483700 & -1.36696200 & 0.24534400 \\ \mathrm{O} & 1.49773900 & -1.57367700 & 0.19510700 \\ \mathrm{O} & 3.60808200 & -2.34800800 & 0.43971000 \\ \mathrm{C} & 3.07338100 & -3.67663800 & 0.58751300 \\ \mathrm{H} & 3.94082600 & -4.31693500 & 0.74535700\end{array}$




$\begin{array}{lrrc}\mathrm{H} & 2.39795900 & -3.72380600 & 1.44499100 \\ \mathrm{H} & 2.53498000 & -3.97456700 & -0.31553200 \\ \mathrm{~N} & 4.64277100 & -0.03353100 & 0.25813200 \\ \mathrm{~N} & 5.77185800 & 0.00806600 & 0.37879500 \\ \mathrm{C} & -4.16912400 & -0.60933200 & -1.11475300 \\ \mathrm{C} & -2.99947000 & -1.10896800 & -1.66544300 \\ \mathrm{C} & -1.82354500 & -1.15907400 & -0.89673300 \\ \mathrm{C} & -1.83212600 & -0.69861400 & 0.43242500 \\ \mathrm{C} & -3.00407500 & -0.19957000 & 0.98417600 \\ \mathrm{C} & -4.16388700 & -0.15770100 & 0.20827100 \\ \mathrm{H} & -5.08717900 & -0.56205200 & -1.68743000 \\ \mathrm{H} & -2.96992400 & -1.46953800 & -2.68837600 \\ \mathrm{H} & -0.91818500 & -0.73210500 & 1.01736200 \\ \mathrm{H} & -3.03584000 & 0.16058200 & 2.00518800 \\ \mathrm{O} & -0.71397800 & -1.65312600 & -1.48895500 \\ \mathrm{H} & 0.03257400 & -1.67111400 & -0.85382800 \\ \mathrm{~N} & -5.39401200 & 0.36843100 & 0.78937100 \\ \mathrm{O} & -6.40238200 & 0.39792700 & 0.07871900 \\ \mathrm{O} & -5.36072300 & 0.75655000 & 1.96038600\end{array}$

10d

$\begin{array}{lrrr}\mathrm{C} & 0.71979500 & 1.21190400 & -0.00013100 \\ \mathrm{C} & -0.67272900 & 1.22337700 & 0.00012000 \\ \mathrm{C} & -1.38382300 & 0.01760800 & 0.00022700 \\ \mathrm{C} & -0.69360200 & -1.19891800 & 0.00009400 \\ \mathrm{C} & 0.70260200 & -1.21411200 & -0.00010800 \\ \mathrm{C} & 1.38916400 & -0.00774200 & -0.00023200 \\ \mathrm{H} & 1.28971900 & 2.13529600 & -0.00021600 \\ \mathrm{H} & -1.22384600 & 2.15818900 & 0.00024300 \\ \mathrm{H} & -1.24308800 & -2.13835200 & 0.00013200 \\ \mathrm{H} & 1.25505000 & -2.14795200 & -0.00019400 \\ \mathrm{O} & -2.75123500 & 0.09521300 & 0.00035100 \\ \mathrm{H} & -3.11810600 & -0.80219400 & 0.00138800 \\ \mathrm{~F} & 2.74241200 & -0.01771100 & -0.00044200\end{array}$

11d

$\begin{array}{lrrr}\text { C } & -0.29212600 & 2.59329900 & 0.70402900 \\ C & -0.87483400 & 1.32924300 & 0.62540100 \\ C & -2.15169900 & 1.17800900 & 0.05989600 \\ C & -2.82969300 & 2.31748800 & -0.40546800 \\ C & -2.24626800 & 3.57839200 & -0.30753400 \\ C & -0.97162300 & 3.72173600 & 0.24315500\end{array}$




$\begin{array}{lrrr}\mathrm{H} & 0.69970800 & 2.69044900 & 1.13615200 \\ \mathrm{H} & -0.33732900 & 0.46922300 & 1.00340800 \\ \mathrm{H} & -3.81437400 & 2.21916700 & -0.85562700 \\ \mathrm{H} & -2.78618000 & 4.44748900 & -0.67328300 \\ \mathrm{H} & -0.51270700 & 4.70392700 & 0.31236200 \\ \mathrm{C} & -2.78991400 & -0.15592000 & -0.02980800 \\ \mathrm{C} & -2.10318500 & -1.43376300 & -0.20028900 \\ \mathrm{O} & -0.89066400 & -1.56686400 & -0.30326100 \\ \mathrm{O} & -2.96289800 & -2.47289400 & -0.24435100 \\ \mathrm{C} & -2.36683000 & -3.77126100 & -0.41824100 \\ \mathrm{H} & -3.20325000 & -4.46968600 & -0.43389400 \\ \mathrm{H} & -1.81066600 & -3.81463200 & -1.35779100 \\ \mathrm{H} & -1.69275400 & -3.99694700 & 0.41175300 \\ \mathrm{~N} & -4.10762100 & -0.21580400 & 0.00004900 \\ \mathrm{~N} & -5.24388400 & -0.24394600 & 0.02350500 \\ \mathrm{C} & 4.80761100 & -0.23752200 & 0.78552100 \\ \mathrm{C} & 3.67202900 & -0.76555300 & 1.39616900 \\ \mathrm{C} & 2.48585300 & -0.92094600 & 0.66716400 \\ \mathrm{C} & 2.44380400 & -0.53916800 & -0.68075200 \\ \mathrm{C} & 3.58127600 & -0.01198500 & -1.29464500 \\ \mathrm{C} & 4.74677300 & 0.13181300 & -0.55372600 \\ \mathrm{H} & 5.73550700 & -0.11181800 & 1.33433100 \\ \mathrm{H} & 3.68848100 & -1.06593300 & 2.43910800 \\ \mathrm{H} & 1.52148200 & -0.65128500 & -1.24353600 \\ \mathrm{H} & 3.56793200 & 0.28866200 & -2.33744400 \\ \mathrm{O} & 1.40521200 & -1.43858600 & 1.32232000 \\ \mathrm{H} & 0.66211900 & -1.54944000 & 0.69732900 \\ \mathrm{~F} & 5.85136900 & 0.64473900 & -1.14896100 \\ & & & \\ & & & \end{array}$

10e

$\begin{array}{lrrr}\mathrm{C} & 0.27442300 & 1.21445700 & -0.00005900 \\ \mathrm{C} & -1.11722000 & 1.22258500 & 0.00016400 \\ \mathrm{C} & -1.82542800 & 0.01551300 & 0.00026700 \\ \mathrm{C} & -1.13088100 & -1.19840100 & 0.00015500 \\ \mathrm{C} & 0.26425900 & -1.20871200 & -0.00002900 \\ \mathrm{C} & 0.95918200 & -0.00195600 & -0.00014500 \\ \mathrm{H} & 0.82695700 & 2.14814200 & -0.00013700 \\ \mathrm{H} & -1.66921100 & 2.15693300 & 0.00026800 \\ \mathrm{H} & -1.67601800 & -2.14038400 & 0.00019600 \\ \mathrm{H} & 0.80442000 & -2.14955200 & -0.00010100 \\ \mathrm{O} & -3.18987400 & 0.08881000 & 0.00038500 \\ \mathrm{H} & -3.55641400 & -0.80896800 & 0.00124500 \\ \mathrm{Cl} & 2.72019100 & -0.01044500 & -0.00039200\end{array}$


11e

$\begin{array}{lrrr}\mathrm{C} & -0.52826200 & 2.52798900 & 0.85331100 \\ \mathrm{C} & -1.17182100 & 1.29937800 & 0.71225900 \\ \mathrm{C} & -2.41675300 & 1.22777900 & 0.06569500 \\ \mathrm{C} & -3.00202300 & 2.41063100 & -0.41691700 \\ \mathrm{C} & -2.35915600 & 3.63583300 & -0.25686100 \\ \mathrm{C} & -1.11594100 & 3.69970200 & 0.37466100 \\ \mathrm{H} & 0.43824400 & 2.56351200 & 1.34776500 \\ \mathrm{H} & -0.70554100 & 0.40469000 & 1.10412400 \\ \mathrm{H} & -3.96038500 & 2.37420800 & -0.92892200 \\ \mathrm{H} & -2.82757600 & 4.53931600 & -0.63745000 \\ \mathrm{H} & -0.61019700 & 4.65384200 & 0.49230400 \\ \mathrm{C} & -3.11808000 & -0.06768500 & -0.09097400 \\ \mathrm{C} & -2.49094700 & -1.37722900 & -0.24714400 \\ \mathrm{O} & -1.28275700 & -1.57352000 & -0.27951600 \\ \mathrm{O} & -3.39918800 & -2.36753400 & -0.36584400 \\ \mathrm{C} & -2.86322900 & -3.69288600 & -0.53288200 \\ \mathrm{H} & -3.73305700 & -4.34360300 & -0.61983400 \\ \mathrm{H} & -2.24947200 & -3.74686400 & -1.43524300 \\ \mathrm{H} & -2.25868900 & -3.97286400 & 0.33336400 \\ \mathrm{~N} & -4.43658200 & -0.05658000 & -0.14315800 \\ \mathrm{~N} & -5.57188200 & -0.02370300 & -0.18981900 \\ \mathrm{C} & 4.40927900 & -0.50726400 & 1.02453000 \\ \mathrm{C} & 3.23950400 & -1.01693700 & 1.58142500 \\ \mathrm{C} & 2.07005300 & -1.10110100 & 0.81475100 \\ \mathrm{C} & 2.08239500 & -0.66565800 & -0.51743100 \\ \mathrm{C} & 3.25436400 & -0.15638200 & -1.07636600 \\ \mathrm{C} & 4.41060100 & -0.07953100 & -0.30336800 \\ \mathrm{H} & 5.31605200 & -0.44309500 & 1.61689400 \\ \mathrm{H} & 3.21679400 & -1.35753400 & 2.61186500 \\ \mathrm{H} & 1.17465100 & -0.72183800 & -1.11132100 \\ \mathrm{H} & 3.26578200 & 0.18113500 & -2.10752100 \\ \mathrm{O} & 0.95605200 & -1.60293700 & 1.41616000 \\ \mathrm{H} & 0.22377900 & -1.65496600 & 0.77003500 \\ \mathrm{Cl} & 5.89271200 & 0.56577200 & -1.01057300\end{array}$

$10 f$

$\begin{array}{llrr}C & -0.32559900 & -1.21042700 & -0.02709100 \\ \mathrm{C} & -1.71416100 & -1.22509100 & -0.00684600 \\ \mathrm{C} & -2.42135100 & -0.01588200 & 0.00424600 \\ \mathrm{C} & -1.72904000 & 1.20038800 & -0.00753000 \\ \mathrm{C} & -0.33666800 & 1.20592900 & -0.02709200\end{array}$




$\begin{array}{lrrr}\mathrm{H} & 0.22255600 & -2.14700100 & -0.04270900 \\ \mathrm{H} & -2.26850800 & -2.15780200 & -0.00313900 \\ \mathrm{H} & -2.27747000 & 2.14029200 & -0.00496400 \\ \mathrm{H} & 0.19876500 & 2.14931500 & -0.04204600 \\ \mathrm{O} & -3.78241100 & -0.08907500 & 0.02246400 \\ \mathrm{H} & -4.15101900 & 0.80823500 & 0.02868000 \\ \mathrm{C} & 1.87232500 & 0.00611000 & 0.00053300 \\ \mathrm{~F} & 2.39080600 & 1.14301800 & -0.51849600 \\ \mathrm{~F} & 2.39577200 & -1.03207900 & -0.69211900 \\ \mathrm{~F} & 2.34976300 & -0.09617300 & 1.26547900\end{array}$

$11 f$

$\begin{array}{lrrr}\mathrm{C} & -1.04171700 & 2.48209000 & 0.94945700 \\ \mathrm{C} & -1.72150700 & 1.27910500 & 0.76465900 \\ \mathrm{C} & -2.94401000 & 1.26092600 & 0.07315400 \\ \mathrm{C} & -3.47097300 & 2.47110200 & -0.40887100 \\ \mathrm{C} & -2.79285500 & 3.67048900 & -0.20510500 \\ \mathrm{C} & -1.57142200 & 3.68099800 & 0.47052200 \\ \mathrm{H} & -0.09302400 & 2.47640000 & 1.47846800 \\ \mathrm{H} & -1.30108300 & 0.36231900 & 1.15721200 \\ \mathrm{H} & -4.41088100 & 2.47644800 & -0.95514100 \\ \mathrm{H} & -3.21625400 & 4.59564800 & -0.58629700 \\ \mathrm{H} & -1.03774500 & 4.61486400 & 0.62222600 \\ \mathrm{C} & -3.68207500 & -0.00690800 & -0.13271700 \\ \mathrm{C} & -3.09467400 & -1.33434000 & -0.28955700 \\ \mathrm{O} & -1.89340200 & -1.57280600 & -0.27521300 \\ \mathrm{O} & -4.03018700 & -2.28929800 & -0.46739800 \\ \mathrm{C} & -3.53416600 & -3.62950100 & -0.64020700 \\ \mathrm{H} & -4.42165900 & -4.24642000 & -0.77913100 \\ \mathrm{H} & -2.88354700 & -3.68690800 & -1.51610600 \\ \mathrm{H} & -2.97883500 & -3.94883800 & 0.24514300 \\ \mathrm{~N} & -4.99651100 & 0.05069000 & -0.23668600 \\ \mathrm{~N} & -6.12717600 & 0.12355100 & -0.32702000 \\ \mathrm{C} & 3.77376700 & -0.77197100 & 1.25571800 \\ \mathrm{C} & 2.57090600 & -1.25372800 & 1.75661700 \\ \mathrm{C} & 1.42714300 & -1.26273900 & 0.94518200 \\ \mathrm{C} & 1.50321800 & -0.78374900 & -0.37147600 \\ \mathrm{C} & 2.71306000 & -0.30439700 & -0.86399000 \\ \mathrm{C} & 3.85488200 & -0.29511500 & -0.05788800 \\ \mathrm{H} & 4.65910500 & -0.77332700 & 1.88355300 \\ \mathrm{H} & 2.49564600 & -1.63326700 & 2.77064400 \\ \mathrm{H} & 0.61587800 & -0.78987100 & -0.99760500\end{array}$




$\begin{array}{lrrr}\mathrm{H} & 2.77164500 & 0.05953000 & -1.88482800 \\ \mathrm{O} & 0.27707500 & -1.74026200 & 1.48620400 \\ \mathrm{H} & -0.43666700 & -1.73518900 & 0.81645800 \\ \mathrm{C} & 5.13841800 & 0.27885900 & -0.57589700 \\ \mathrm{~F} & 6.22146500 & -0.29825500 & -0.00383300 \\ \mathrm{~F} & 5.25949900 & 0.12525100 & -1.91594800 \\ \mathrm{~F} & 5.24207800 & 1.61090700 & -0.33299800\end{array}$

\section{$10 \mathrm{~g}$}

$\begin{array}{lrrr}\mathrm{C} & 0.06075800 & 1.37953800 & 0.00000100 \\ \mathrm{C} & -1.31417800 & 1.19495700 & 0.00000200 \\ \mathrm{C} & -1.84966700 & -0.10007500 & -0.00000500 \\ \mathrm{C} & -0.98941200 & -1.19599400 & -0.00001500 \\ \mathrm{C} & 0.39901800 & -1.01274400 & -0.00001700 \\ \mathrm{C} & 0.93108000 & 0.27845400 & -0.00000900 \\ \mathrm{H} & 0.48854600 & 2.37727900 & 0.00000800 \\ \mathrm{H} & -1.99107100 & 2.04355300 & 0.00001000 \\ \mathrm{H} & -1.39346600 & -2.20701300 & -0.00002100 \\ \mathrm{H} & 1.04253000 & -1.88499800 & -0.00002700 \\ \mathrm{O} & -3.21742700 & -0.21808700 & -0.00000200 \\ \mathrm{H} & -3.44912400 & -1.15952600 & -0.00001000 \\ \mathrm{O} & 2.26895100 & 0.57692500 & -0.00001300 \\ \mathrm{C} & 3.18801200 & -0.49985400 & 0.00004300 \\ \mathrm{H} & 3.07738100 & -1.12877200 & -0.89435600 \\ \mathrm{H} & 4.18203600 & -0.04817400 & 0.00007400 \\ \mathrm{H} & 3.07730600 & -1.12874000 & 0.89445400\end{array}$

$11 g$

$\begin{array}{lrrr}\mathrm{C} & -0.46083100 & 2.48405800 & 0.86970200 \\ \mathrm{C} & -1.12829000 & 1.27108700 & 0.70577900 \\ \mathrm{C} & -2.38047800 & 1.23863000 & 0.07027600 \\ \mathrm{C} & -2.94886500 & 2.44337500 & -0.37714600 \\ \mathrm{C} & -2.28218700 & 3.65247800 & -0.19359600 \\ \mathrm{C} & -1.03156900 & 3.67778700 & 0.42613300 \\ \mathrm{H} & 0.51118700 & 2.48843100 & 1.35445700 \\ \mathrm{H} & -0.67503900 & 0.35954000 & 1.07354900 \\ \mathrm{H} & -3.91296900 & 2.43673400 & -0.87965800 \\ \mathrm{H} & -2.73793200 & 4.57359100 & -0.54652600 \\ \mathrm{H} & -0.50740800 & 4.61955600 & 0.56236400 \\ \mathrm{C} & -3.10641800 & -0.03996000 & -0.11097500 \\ \mathrm{C} & -2.50219700 & -1.35521600 & -0.31243400 \\ \mathrm{O} & -1.29909900 & -1.56998200 & -0.36965000\end{array}$




$\begin{array}{lrrr}\mathrm{O} & -3.43014900 & -2.32720200 & -0.44254400 \\ \mathrm{C} & -2.91768800 & -3.65574000 & -0.64960000 \\ \mathrm{H} & -3.79866400 & -4.29096800 & -0.73998600 \\ \mathrm{H} & -2.31716700 & -3.69718000 & -1.56154900 \\ \mathrm{H} & -2.30527200 & -3.96637800 & 0.20056300 \\ \mathrm{~N} & -4.42460000 & -0.00544000 & -0.14281800 \\ \mathrm{~N} & -5.56008800 & 0.04757300 & -0.17153900 \\ \mathrm{C} & 4.36886200 & -0.56794000 & 1.22797900 \\ \mathrm{C} & 3.17049900 & -1.07120600 & 1.71584400 \\ \mathrm{C} & 2.04388500 & -1.14633800 & 0.88435300 \\ \mathrm{C} & 2.14107600 & -0.70640600 & -0.43699300 \\ \mathrm{C} & 3.34971600 & -0.20087200 & -0.93075500 \\ \mathrm{C} & 4.47032100 & -0.12839400 & -0.09996200 \\ \mathrm{H} & 5.24774900 & -0.50658700 & 1.86268200 \\ \mathrm{H} & 3.08802500 & -1.41496300 & 2.74251700 \\ \mathrm{H} & 1.27048400 & -0.75347400 & -1.08562800 \\ \mathrm{H} & 3.39472000 & 0.13229000 & -1.96167200 \\ \mathrm{O} & 0.88800800 & -1.64477400 & 1.42297200 \\ \mathrm{H} & 0.19923400 & -1.69122600 & 0.73214200 \\ \mathrm{O} & 5.70104700 & 0.34986500 & -0.47888200 \\ \mathrm{C} & 5.85141200 & 0.80105700 & -1.81088900 \\ \mathrm{H} & 5.18299600 & 1.64483400 & -2.03366000 \\ \mathrm{H} & 6.88823900 & 1.13148400 & -1.90356200 \\ \mathrm{H} & 5.66268200 & -0.00227200 & -2.53698800\end{array}$

$10 \mathrm{~h}$

$\begin{array}{lrrr}\text { C } & -0.68510600 & -1.18333700 & -0.38137500 \\ \text { C } & -2.04204800 & -1.22271200 & -0.07688800 \\ \text { C } & -2.75610300 & -0.02938800 & 0.08834300 \\ \text { C } & -2.10564400 & 1.20155800 & -0.05359900 \\ \text { C } & -0.74479900 & 1.24063200 & -0.35599900 \\ \text { C } & -0.04807400 & 0.04874600 & -0.51597000 \\ \text { H } & -0.11821700 & -2.09784300 & -0.51700100 \\ \text { H } & -2.56426500 & -2.16727300 & 0.03391400 \\ \text { H } & -2.65941500 & 2.12986500 & 0.07109700 \\ \text { H } & -0.22559600 & 2.18600300 & -0.47175400 \\ \text { O } & -4.08575100 & -0.13274600 & 0.38348400 \\ \text { H } & -4.46264300 & 0.75634300 & 0.47286600 \\ \text { O } & 1.30658600 & 0.09696800 & -0.89622800 \\ \text { C } & 2.22703100 & 0.00144000 & 0.09336900 \\ \text { F } & 2.09422500 & 0.97497200 & 1.01601400 \\ \text { F } & 3.43422800 & 0.10445900 & -0.46343300 \\ \text { F } & 2.15953800 & -1.17526400 & 0.75025800\end{array}$




$\begin{array}{lrrr}\mathrm{C} & -0.93348400 & 2.29297300 & 1.03558600 \\ \mathrm{C} & -1.77504800 & 1.20496700 & 0.80850400 \\ \mathrm{C} & -2.99416100 & 1.38696500 & 0.13495700 \\ \mathrm{C} & -3.35217000 & 2.67860400 & -0.28692100 \\ \mathrm{C} & -2.51261100 & 3.76239000 & -0.04103200 \\ \mathrm{C} & -1.29616200 & 3.57394200 & 0.61711500 \\ \mathrm{H} & 0.00978700 & 2.13231100 & 1.54961500 \\ \mathrm{H} & -1.48267000 & 0.22186400 & 1.15425500 \\ \mathrm{H} & -4.28677300 & 2.83817500 & -0.81883900 \\ \mathrm{H} & -2.80670100 & 4.75334000 & -0.37581200 \\ \mathrm{H} & -0.63706200 & 4.41747200 & 0.80139400 \\ \mathrm{C} & -3.90384600 & 0.24425900 & -0.11209200 \\ \mathrm{C} & -3.50841500 & -1.14393700 & -0.33231800 \\ \mathrm{O} & -2.35177100 & -1.54545500 & -0.35361800 \\ \mathrm{O} & -4.57019400 & -1.95311000 & -0.52521200 \\ \mathrm{C} & -4.26703300 & -3.34097300 & -0.75712900 \\ \mathrm{H} & -5.23334700 & -3.82425200 & -0.89932700 \\ \mathrm{H} & -3.64461600 & -3.45320500 & -1.64807700 \\ \mathrm{H} & -3.74681300 & -3.76760100 & 0.10402900 \\ \mathrm{~N} & -5.19856000 & 0.48786000 & -0.18848200 \\ \mathrm{~N} & -6.30913300 & 0.72085900 & -0.25476500 \\ \mathrm{C} & 3.41361900 & -1.55059900 & 1.05785100 \\ \mathrm{C} & 2.16873700 & -1.92133800 & 1.55742600 \\ \mathrm{C} & 1.01329000 & -1.72170300 & 0.78888400 \\ \mathrm{C} & 1.11506900 & -1.14626100 & -0.48634400 \\ \mathrm{C} & 2.36292500 & -0.77496500 & -0.98481300 \\ \mathrm{C} & 3.49858800 & -0.97836000 & -0.20898000 \\ \mathrm{~F} & 4.31665300 & -1.70322800 & 1.63926500 \\ \mathrm{H} & 2.07223000 & -2.37099100 & 2.54044600 \\ \mathrm{H} & 0.21864900 & -0.98760600 & -1.07838500 \\ \mathrm{H} & 2.45919900 & -0.32966400 & -1.96954900 \\ \mathrm{O} & -0.17591200 & -2.09811000 & 1.33261300 \\ \mathrm{H} & -0.89725900 & -1.96037800 & 0.68667300 \\ \mathrm{O} & 4.76468700 & -0.66504400 & -0.74769300 \\ \mathrm{~F} & 5.26067900 & 0.56162400 & -0.46869500 \\ \mathrm{H} & 5.44675700 & 0.75865200 & 0.85383200 \\ \mathrm{H} & & & \end{array}$

$10 \mathbf{i}$

C $\quad 0.35492000 \quad-1.12374600 \quad-0.00002000$ 


$\begin{array}{lrrr}\mathrm{C} & 1.73478400 & -1.27690100 & -0.00011000 \\ \mathrm{C} & 2.55947200 & -0.14398400 & -0.00016900 \\ \mathrm{C} & 1.99368700 & 1.13814900 & -0.00013700 \\ \mathrm{C} & 0.61166700 & 1.28150400 & -0.00004100 \\ \mathrm{C} & -0.22339700 & 0.15623000 & 0.00001700 \\ \mathrm{H} & -0.28574300 & -1.99839700 & 0.00002700 \\ \mathrm{H} & 2.19453400 & -2.25990300 & -0.00013200 \\ \mathrm{H} & 2.63653500 & 2.01662300 & -0.00019100 \\ \mathrm{H} & 0.15652800 & 2.26644000 & -0.00001300 \\ \mathrm{O} & 3.90616600 & -0.35162100 & -0.00027400 \\ \mathrm{H} & 4.36058600 & 0.50560700 & -0.00015800 \\ \mathrm{C} & -1.69047100 & 0.37732300 & 0.00011800 \\ \mathrm{O} & -2.22682700 & 1.46970400 & 0.00015200 \\ \mathrm{O} & -2.39055100 & -0.78424500 & 0.00015400 \\ \mathrm{C} & -3.81718700 & -0.63604900 & 0.00024400 \\ \mathrm{H} & -4.21670200 & -1.65090500 & 0.00025800 \\ \mathrm{H} & -4.14839300 & -0.09267400 & 0.88941300 \\ \mathrm{H} & -4.14850400 & -0.09265400 & -0.88887000\end{array}$

$11 i$

$\begin{array}{lrrr}\mathrm{C} & -1.03696200 & 2.47772200 & 0.92074000 \\ \mathrm{C} & -1.73669100 & 1.28569400 & 0.73988200 \\ \mathrm{C} & -2.98038300 & 1.29207400 & 0.08715500 \\ \mathrm{C} & -3.50804500 & 2.51530700 & -0.35964700 \\ \mathrm{C} & -2.80961900 & 3.70362600 & -0.15955000 \\ \mathrm{C} & -1.56732400 & 3.68976100 & 0.47684700 \\ \mathrm{H} & -0.07164100 & 2.45263700 & 1.41811400 \\ \mathrm{H} & -1.31552600 & 0.35800600 & 1.10512400 \\ \mathrm{H} & -4.46458800 & 2.53955600 & -0.87572800 \\ \mathrm{H} & -3.23366900 & 4.63927500 & -0.51346700 \\ \mathrm{H} & -1.01776200 & 4.61498400 & 0.62481000 \\ \mathrm{C} & -3.73877900 & 0.03561700 & -0.11405400 \\ \mathrm{C} & -3.16987400 & -1.29458900 & -0.31182600 \\ \mathrm{O} & -1.97127200 & -1.54323300 & -0.34424100 \\ \mathrm{O} & -4.12024900 & -2.23851800 & -0.47002700 \\ \mathrm{C} & -3.64268100 & -3.57999700 & -0.68037800 \\ \mathrm{H} & -4.53995200 & -4.18696600 & -0.79902200 \\ \mathrm{H} & -3.02211900 & -3.62911600 & -1.57829700 \\ \mathrm{H} & -3.06106100 & -3.91821300 & 0.18078900 \\ \mathrm{~N} & -5.05512500 & 0.10784300 & -0.17159000 \\ \mathrm{~N} & -6.18751600 & 0.19332600 & -0.22173000 \\ \mathrm{C} & 3.73036900 & -0.86343800 & 1.17499000 \\ \mathrm{C} & 2.52414400 & -1.35110100 & 1.65842800\end{array}$




$\begin{array}{lrrr}\mathrm{C} & 1.37775700 & -1.31400800 & 0.84973000 \\ \mathrm{C} & 1.45357400 & -0.78014400 & -0.44739900 \\ \mathrm{C} & 2.66579800 & -0.29564400 & -0.92120300 \\ \mathrm{C} & 3.81758900 & -0.32887000 & -0.12132600 \\ \mathrm{H} & 4.61580500 & -0.89376300 & 1.80028700 \\ \mathrm{H} & 2.44409100 & -1.76776900 & 2.65762100 \\ \mathrm{H} & 0.56190900 & -0.74711900 & -1.06703800 \\ \mathrm{H} & 2.74143200 & 0.11976700 & -1.92097500 \\ \mathrm{O} & 0.22426700 & -1.79839200 & 1.37481900 \\ \mathrm{H} & -0.49212900 & -1.75726400 & 0.70903700 \\ \mathrm{C} & 5.07742600 & 0.20573500 & -0.68738300 \\ \mathrm{O} & 6.11765200 & 0.11965600 & 0.18270500 \\ \mathrm{C} & 7.36592100 & 0.62088600 & -0.31088900 \\ \mathrm{H} & 7.68099400 & 0.06756400 & -1.20006100 \\ \mathrm{H} & 7.28340800 & 1.68070000 & -0.56796000 \\ \mathrm{H} & 8.08060000 & 0.47880600 & 0.50101000 \\ \mathrm{O} & 5.19652300 & 0.67660500 & -1.80396900\end{array}$

$10 k$

$\begin{array}{lrrr}\mathrm{C} & -1.20198000 & -0.21604400 & 0.00857400 \\ \mathrm{C} & -0.02137500 & -0.97120400 & 0.00732500 \\ \mathrm{C} & 1.22746500 & -0.34564100 & -0.00080800 \\ \mathrm{C} & 1.31633600 & 1.05092800 & -0.00559700 \\ \mathrm{C} & 0.14504500 & 1.80160200 & -0.00171700 \\ \mathrm{C} & -1.10664500 & 1.17936500 & 0.00634900 \\ \mathrm{H} & -0.07717500 & -2.05990400 & 0.01349400 \\ \mathrm{H} & 2.29579400 & 1.51834700 & -0.00907400 \\ \mathrm{H} & 0.20718300 & 2.88680000 & -0.00192500 \\ \mathrm{O} & 2.40177500 & -1.05000000 & -0.00156200 \\ \mathrm{H} & 2.19779600 & -1.99809400 & 0.00271300 \\ \mathrm{H} & -2.01229600 & 1.78022000 & 0.01148900 \\ \mathrm{C} & -2.54604100 & -0.90746600 & -0.00719100 \\ \mathrm{H} & -2.76801000 & -1.32542700 & -0.99802500 \\ \mathrm{H} & -3.35380500 & -0.21319000 & 0.24462100 \\ \mathrm{H} & -2.58052500 & -1.73799200 & 0.70759000\end{array}$

$11 k$

$\begin{array}{llll}\mathrm{C} & 0.12705700 & 2.53533500 & -0.81200000 \\ \mathrm{C} & 0.73160000 & 1.28390100 & -0.70221200 \\ \mathrm{C} & 2.00710800 & 1.16745500 & -0.12574600 \\ \mathrm{C} & 2.66249100 & 2.32865100 & 0.31796800 \\ \mathrm{C} & 2.05785300 & 3.57667700 & 0.18825900\end{array}$




$\begin{array}{lrrr}\mathrm{C} & 0.78410600 & 3.68540300 & -0.37258100 \\ \mathrm{H} & -0.86419900 & 2.60417900 & -1.25072900 \\ \mathrm{H} & 0.21189800 & 0.40678500 & -1.06521200 \\ \mathrm{H} & 3.64602700 & 2.25757100 & 0.77577800 \\ \mathrm{H} & 2.58050100 & 4.46306000 & 0.53736600 \\ \mathrm{H} & 0.30873000 & 4.65775000 & -0.46619700 \\ \mathrm{C} & 2.66631500 & -0.15339300 & -0.00226300 \\ \mathrm{C} & 1.99791000 & -1.43868600 & 0.19018200 \\ \mathrm{O} & 0.78782400 & -1.58940400 & 0.28865400 \\ \mathrm{O} & 2.87479200 & -2.46281400 & 0.26030700 \\ \mathrm{C} & 2.29766100 & -3.76646000 & 0.45538300 \\ \mathrm{H} & 3.14439800 & -4.45182500 & 0.48820500 \\ \mathrm{H} & 1.73716000 & -3.80102800 & 1.39277700 \\ \mathrm{H} & 1.63116200 & -4.01772500 & -0.37339600 \\ \mathrm{~N} & 3.98466100 & -0.19243300 & -0.02075200 \\ \mathrm{~N} & 5.12159800 & -0.20248300 & -0.03467200 \\ \mathrm{C} & -4.87070600 & -0.19974000 & -1.02308000 \\ \mathrm{C} & -3.73879700 & -0.78742200 & -1.57952800 \\ \mathrm{C} & -2.58960200 & -0.93940000 & -0.79298400 \\ \mathrm{C} & -2.58656300 & -0.49880300 & 0.53525000 \\ \mathrm{C} & -3.72777300 & 0.08930200 & 1.09606000 \\ \mathrm{C} & -4.87183400 & 0.23831500 & 0.30417900 \\ \mathrm{H} & -5.76384800 & -0.07894600 & -1.63134000 \\ \mathrm{H} & -3.72171500 & -1.13222300 & -2.60881400 \\ \mathrm{O} & -1.49665600 & -1.51297100 & -1.37685700 \\ \mathrm{H} & -0.77887000 & -1.60047000 & -0.71983400 \\ \mathrm{H} & -5.76311700 & 0.69666200 & 0.72542200 \\ \mathrm{H} & -1.68329700 & -0.61318200 & 1.13073500 \\ \mathrm{C} & -3.71656500 & 0.53638100 & 2.54046000 \\ \mathrm{H} & -3.79360100 & -0.31978700 & 3.22395300 \\ \mathrm{H} & -2.78897100 & 1.06433100 & 2.79049000 \\ \mathrm{H} & -4.55532200 & 1.20568300 & 2.75784600\end{array}$

101

$\begin{array}{lrrr}\mathrm{C} & 0.40235500 & -0.86201400 & -0.00008800 \\ \mathrm{C} & 1.74107100 & -0.46794600 & 0.00002500 \\ \mathrm{C} & 2.07175500 & 0.89499600 & 0.00010200 \\ \mathrm{C} & 1.06417600 & 1.85592700 & 0.00005800 \\ \mathrm{C} & -0.28178200 & 1.48544000 & 0.00002700 \\ \mathrm{C} & -0.57987800 & 0.12660000 & -0.00002800 \\ \mathrm{H} & 0.10878100 & -1.90603700 & -0.00024700 \\ \mathrm{H} & 1.32981800 & 2.90873400 & 0.00011600 \\ \mathrm{H} & -1.08173500 & 2.21410600 & 0.00005300\end{array}$




$\begin{array}{lrrr}\mathrm{N} & -1.99108600 & -0.29588100 & -0.00005800 \\ \mathrm{O} & -2.22654700 & -1.50470900 & 0.00022800 \\ \mathrm{O} & -2.85168900 & 0.58291000 & -0.00015600 \\ \mathrm{H} & 3.11983500 & 1.17627100 & 0.00022100 \\ \mathrm{O} & 2.77065800 & -1.36031300 & -0.00022600 \\ \mathrm{H} & 2.41535500 & -2.26303000 & 0.00091900\end{array}$

111

$\begin{array}{lrrr}\mathrm{C} & 3.12081800 & -2.65077400 & -0.39097100 \\ \mathrm{C} & 2.52778900 & -1.38928700 & -0.37939200 \\ \mathrm{C} & 3.30241400 & -0.24806000 & -0.11524000 \\ \mathrm{C} & 4.67983600 & -0.40199300 & 0.11736900 \\ \mathrm{C} & 5.26752300 & -1.66431800 & 0.08664600 \\ \mathrm{C} & 4.48972000 & -2.79632700 & -0.16269200 \\ \mathrm{H} & 2.50278900 & -3.52224700 & -0.58742000 \\ \mathrm{H} & 1.46690800 & -1.29741900 & -0.57172300 \\ \mathrm{H} & 5.29571400 & 0.46722300 & 0.33396200 \\ \mathrm{H} & 6.33406200 & -1.76199200 & 0.26967600 \\ \mathrm{H} & 4.94652600 & -3.78176800 & -0.17818100 \\ \mathrm{C} & 2.69049200 & 1.10034400 & -0.09958000 \\ \mathrm{C} & 1.32365200 & 1.43259100 & 0.29868400 \\ \mathrm{O} & 0.49283100 & 0.62094500 & 0.68997000 \\ \mathrm{O} & 1.07358000 & 2.75014300 & 0.19680300 \\ \mathrm{C} & -0.24284500 & 3.19043800 & 0.59170000 \\ \mathrm{H} & -0.21903200 & 4.27512400 & 0.49077500 \\ \mathrm{H} & -1.00826100 & 2.76371900 & -0.05971700 \\ \mathrm{H} & -0.44460600 & 2.90520700 & 1.62670100 \\ \mathrm{~N} & 3.45104000 & 2.12667400 & -0.43104800 \\ \mathrm{~N} & 4.12520000 & 2.99613000 & -0.71631400 \\ \mathrm{C} & -4.21019200 & -2.57327100 & -0.20375200 \\ \mathrm{C} & -2.87615900 & -2.76938300 & 0.14761000 \\ \mathrm{C} & -2.03456500 & -1.66805100 & 0.37132400 \\ \mathrm{C} & -2.54454700 & -0.37226400 & 0.24126000 \\ \mathrm{C} & -3.88085100 & -0.21355900 & -0.11423500 \\ \mathrm{C} & -4.73723000 & -1.28842200 & -0.34144600 \\ \mathrm{H} & -4.84993400 & -3.43425100 & -0.37389400 \\ \mathrm{H} & -2.46418000 & -3.76786300 & 0.25495900 \\ \mathrm{H} & -0.74520600 & -1.91177500 & 0.71022700 \\ \mathrm{H} & -0.26333900 & -1.06679100 & 0.82563000 \\ & -5.76916000 & -1.11297400 & -0.61552900 \\ \mathrm{H} & -1.91504900 & 0.48980600 & 0.41291900 \\ \mathrm{H} & -3.40105400 & 1.15498500 & -0.25847400 \\ \mathrm{H} & & 2.09418300 & -0.09157600\end{array}$


$\begin{array}{llll}\text { O } & -5.59061000 & 1.29321800 & -0.53930200\end{array}$

$10 \mathrm{~m}$

$\begin{array}{lrrr}\mathrm{C} & -1.19429000 & -0.31716900 & -0.00000200 \\ \mathrm{C} & -0.00151500 & -1.02512500 & 0.00000500 \\ \mathrm{C} & 1.19673200 & -0.30333900 & -0.00000100 \\ \mathrm{C} & 1.18080700 & 1.09667600 & -0.00000700 \\ \mathrm{C} & -0.04108800 & 1.77102900 & 0.00000100 \\ \mathrm{C} & -1.24867900 & 1.07399300 & 0.00000000 \\ \mathrm{H} & 0.00511400 & -2.10878800 & 0.00002100 \\ \mathrm{H} & 2.11594500 & 1.65243900 & -0.00002800 \\ \mathrm{H} & -0.04954100 & 2.85740400 & 0.00000100 \\ \mathrm{O} & 2.35440400 & -1.02837200 & -0.00003400 \\ \mathrm{H} & 3.10780300 & -0.41781100 & 0.00026000 \\ \mathrm{H} & -2.20840900 & 1.57816800 & 0.00000100 \\ \mathrm{~F} & -2.35088300 & -1.01231400 & 0.00000300\end{array}$

$11 \mathrm{~m}$

$\begin{array}{lrrr}\mathrm{C} & 0.35796200 & 2.64215900 & -0.51437300 \\ \mathrm{C} & 0.88941100 & 1.35337700 & -0.50892300 \\ \mathrm{C} & 2.21029900 & 1.13951600 & -0.08176100 \\ \mathrm{C} & 2.98244900 & 2.24231900 & 0.32114700 \\ \mathrm{C} & 2.44818900 & 3.52831600 & 0.29675300 \\ \mathrm{C} & 1.13082000 & 3.73387800 & -0.11650100 \\ \mathrm{H} & -0.66816200 & 2.78771500 & -0.83962700 \\ \mathrm{H} & 0.27895700 & 0.52218200 & -0.83688100 \\ \mathrm{H} & 4.00341800 & 2.09573500 & 0.66473000 \\ \mathrm{H} & 3.06111500 & 4.36819000 & 0.61224000 \\ \mathrm{H} & 0.71101500 & 4.73572900 & -0.12854800 \\ \mathrm{C} & 2.79476900 & -0.22155400 & -0.07296800 \\ \mathrm{C} & 2.07247100 & -1.47221300 & 0.14506800 \\ \mathrm{O} & 0.87039700 & -1.55589600 & 0.36109700 \\ \mathrm{O} & 2.88649300 & -2.54698400 & 0.09357600 \\ \mathrm{C} & 2.25341200 & -3.82248100 & 0.30294100 \\ \mathrm{H} & 3.05829400 & -4.55445900 & 0.23968400 \\ \mathrm{H} & 1.77687900 & -3.85687600 & 1.28555500 \\ \mathrm{H} & 1.50330600 & -4.00841900 & -0.46976200 \\ \mathrm{~N} & 4.09950200 & -0.33690600 & -0.23222500 \\ \mathrm{~N} & 5.22570300 & -0.41285100 & -0.36728400 \\ \mathrm{C} & -4.82587500 & -0.02741300 & -0.23321400 \\ \mathrm{C} & -3.78886800 & -0.60550800 & -0.95030800 \\ \mathrm{C} & -2.55763600 & -0.79377400 & -0.30999100\end{array}$




$\begin{array}{lrrr}\text { C } & -2.39112700 & -0.39836300 & 1.02602400 \\ \mathrm{C} & -3.46099400 & 0.17830100 & 1.71061000 \\ \mathrm{C} & -4.69702000 & 0.37418400 & 1.09253600 \\ \mathrm{H} & -3.92682400 & -0.90843300 & -1.98194300 \\ \mathrm{O} & -1.55701900 & -1.35794300 & -1.03995200 \\ \mathrm{H} & -0.76548200 & -1.48103300 & -0.47907400 \\ \mathrm{H} & -5.53957100 & 0.82066400 & 1.60870500 \\ \mathrm{H} & -1.43079000 & -0.54054800 & 1.51255300 \\ \mathrm{H} & -3.32728800 & 0.48257900 & 2.74525000 \\ \mathrm{~F} & -6.01331600 & 0.15193300 & -0.85514900\end{array}$

10n

$\begin{array}{lrrr}\mathrm{C} & -0.83552700 & 0.04924700 & -0.00000900 \\ \mathrm{C} & 0.17243000 & -0.90857900 & 0.00001100 \\ \mathrm{C} & 1.50521200 & -0.48141500 & 0.00001900 \\ \mathrm{C} & 1.81031900 & 0.88390500 & 0.00000800 \\ \mathrm{C} & 0.77604700 & 1.81968500 & -0.00000800 \\ \mathrm{C} & -0.55911300 & 1.41740400 & -0.00001800 \\ \mathrm{H} & -0.05235400 & -1.96870000 & 0.00002200 \\ \mathrm{H} & 2.84811900 & 1.21086900 & 0.00001100 \\ \mathrm{H} & 1.01508300 & 2.87944500 & -0.00001500 \\ \mathrm{O} & 2.46360400 & -1.45415800 & 0.00002900 \\ \mathrm{H} & 3.33895800 & -1.03665400 & 0.00011000 \\ \mathrm{H} & -1.36611800 & 2.14099800 & -0.00003200 \\ \mathrm{Cl} & -2.51227800 & -0.48671700 & -0.00002000\end{array}$

11n

$\begin{array}{lrrr}\mathrm{C} & 0.61371200 & 2.61815800 & -1.04308600 \\ \mathrm{C} & 1.11180900 & 1.32694600 & -0.87502100 \\ \mathrm{C} & 2.28608400 & 1.11687600 & -0.13339300 \\ \mathrm{C} & 2.94883300 & 2.22701800 & 0.41789900 \\ \mathrm{C} & 2.45160500 & 3.51443300 & 0.23197000 \\ \mathrm{C} & 1.27810500 & 3.71645400 & -0.49624000 \\ \mathrm{H} & -0.30041000 & 2.76018700 & -1.61247800 \\ \mathrm{H} & 0.58554700 & 0.49155400 & -1.31752600 \\ \mathrm{H} & 3.85310800 & 2.08605000 & 1.00458600 \\ \mathrm{H} & 2.97842000 & 4.35878000 & 0.66797400 \\ \mathrm{H} & 0.88526800 & 4.71968200 & -0.63464000 \\ \mathrm{C} & 2.83723000 & -0.24529100 & 0.05422600 \\ \mathrm{C} & 2.08106600 & -1.49438000 & 0.08699200 \\ \mathrm{O} & 0.86707200 & -1.58246600 & -0.04368900 \\ \mathrm{O} & 2.87518600 & -2.56563800 & 0.29156000\end{array}$




$\begin{array}{lrrr}\mathrm{C} & 2.20684400 & -3.83912800 & 0.34848600 \\ \mathrm{H} & 2.99624000 & -4.56774500 & 0.53165400 \\ \mathrm{H} & 1.47483100 & -3.85098900 & 1.15950200 \\ \mathrm{H} & 1.70211000 & -4.04870100 & -0.59787400 \\ \mathrm{~N} & 4.13540000 & -0.36375200 & 0.26073300 \\ \mathrm{~N} & 5.25522400 & -0.44117600 & 0.43966500 \\ \mathrm{C} & -4.69453300 & -0.05354900 & -1.34982200 \\ \mathrm{C} & -3.54577000 & -0.56160800 & -1.94891800 \\ \mathrm{C} & -2.40311400 & -0.79375500 & -1.17170100 \\ \mathrm{C} & -2.41741100 & -0.51097500 & 0.20160000 \\ \mathrm{C} & -3.58488400 & -0.00568700 & 0.76847800 \\ \mathrm{C} & -4.73378900 & 0.23191300 & 0.01789900 \\ \mathrm{H} & -5.57968700 & 0.12340700 & -1.95498500 \\ \mathrm{H} & -3.51164400 & -0.78804000 & -3.00966800 \\ \mathrm{O} & -1.30260700 & -1.28951300 & -1.79880900 \\ \mathrm{H} & -0.59054900 & -1.45044200 & -1.14808600 \\ \mathrm{H} & -5.62758800 & 0.62559600 & 0.48815900 \\ \mathrm{H} & -1.53350500 & -0.67921200 & 0.80729100 \\ \mathrm{Cl} & -3.59990800 & 0.34533900 & 2.49791800\end{array}$

100

$\begin{array}{lrrr}\text { C } & -0.27673600 & 0.19129000 & -0.03744800 \\ \text { C } & 0.67870600 & -0.82834000 & -0.02753200 \\ \text { C } & 2.03608000 & -0.50052000 & -0.00112300 \\ \text { C } & 2.43339200 & 0.84217800 & 0.01452100 \\ \text { C } & 1.46963400 & 1.84572000 & 0.00257100 \\ \text { C } & 0.10882700 & 1.53192900 & -0.02295200 \\ \text { H } & 0.36373600 & -1.86841900 & -0.04940400 \\ \text { H } & 3.49348800 & 1.07393100 & 0.03251200 \\ \text { H } & 1.78192700 & 2.88606300 & 0.01011500 \\ \text { O } & 3.02150800 & -1.44513600 & 0.00587000 \\ \text { H } & 2.61883500 & -2.32750200 & -0.00488200 \\ \text { H } & -0.64117300 & 2.31443200 & -0.03932700 \\ \text { C } & -1.73697600 & -0.17170900 & -0.00157800 \\ \text { F } & -1.98465400 & -1.33268900 & -0.65151200 \\ \text { F } & -2.18024400 & -0.33553900 & 1.26758200 \\ \text { F } & -2.50915000 & 0.78148400 & -0.56659600\end{array}$

110

\begin{tabular}{|c|c|c|c|}
\hline$C$ & 1.16169200 & 2.60376700 & -0.49928400 \\
\hline & 1.74365400 & 1.33707700 & -0.51770300 \\
\hline & 3.07991700 & 1.17117500 & -0.1178 \\
\hline
\end{tabular}




$\begin{array}{lrrr}\mathrm{C} & 3.81514700 & 2.29968000 & 0.28307900 \\ \mathrm{C} & 3.23009300 & 3.56360700 & 0.28289600 \\ \mathrm{C} & 1.89815300 & 3.72117900 & -0.10386600 \\ \mathrm{H} & 0.12488600 & 2.71234600 & -0.80457400 \\ \mathrm{H} & 1.15969900 & 0.48575100 & -0.84238900 \\ \mathrm{H} & 4.84746200 & 2.19056800 & 0.60600500 \\ \mathrm{H} & 3.81523600 & 4.42375200 & 0.59642600 \\ \mathrm{H} & 1.43901700 & 4.70563300 & -0.09739200 \\ \mathrm{C} & 3.71879500 & -0.16518000 & -0.13627400 \\ \mathrm{C} & 3.05306600 & -1.44753400 & 0.07498800 \\ \mathrm{O} & 1.85890700 & -1.58500100 & 0.30889400 \\ \mathrm{O} & 3.90959400 & -2.48640700 & -0.00448500 \\ \mathrm{C} & 3.33497700 & -3.79038300 & 0.19876300 \\ \mathrm{H} & 4.16895400 & -4.48639400 & 0.11169900 \\ \mathrm{H} & 2.87825800 & -3.85733200 & 1.18906900 \\ \mathrm{H} & 2.57973400 & -3.99833100 & -0.56320200 \\ \mathrm{~N} & 5.02466600 & -0.22479700 & -0.31797800 \\ \mathrm{~N} & 6.15051200 & -0.25242300 & -0.47173000 \\ \mathrm{C} & -3.87913800 & -0.11830900 & 0.18514400 \\ \mathrm{C} & -2.88275300 & -0.67462900 & -0.61451000 \\ \mathrm{C} & -1.60299500 & -0.87862200 & -0.08485700 \\ \mathrm{C} & -1.33311400 & -0.52150000 & 1.24357700 \\ \mathrm{C} & -2.34329000 & 0.03237300 & 2.02995200 \\ \mathrm{C} & -3.62051600 & 0.23975600 & 1.51266100 \\ \mathrm{H} & -3.07705900 & -0.94881800 & -1.64516800 \\ \mathrm{O} & -0.66360600 & -1.41459400 & -0.90967500 \\ \mathrm{H} & 0.17614000 & -1.53865300 & -0.42369700 \\ \mathrm{H} & -4.40253200 & 0.67919600 & 2.12205300 \\ \mathrm{H} & -0.33568600 & -0.67248800 & 1.64649700 \\ \mathrm{H} & -2.12581700 & 0.31065000 & 3.05757500 \\ \mathrm{C} & -5.26861900 & 0.04765800 & -0.36752200 \\ \mathrm{~F} & -6.05980600 & -1.01073400 & -0.06653500 \\ \mathrm{~F} & -5.88249900 & 1.14354800 & 0.13643000 \\ \mathrm{~F} & -5.27370500 & 0.16740400 & -1.71414000\end{array}$

\section{$10 p$}

$\begin{array}{lrrr}\mathrm{C} & 0.82069100 & 0.37458500 & -0.00001000 \\ \mathrm{C} & 0.02452100 & -0.77270900 & -0.00000400 \\ \mathrm{C} & -1.36972800 & -0.63758100 & -0.00003900 \\ \mathrm{C} & -1.96915600 & 0.62251100 & -0.00005900 \\ \mathrm{C} & -1.15300600 & 1.75850600 & -0.00002000 \\ \mathrm{C} & 0.23106300 & 1.64990500 & 0.00002400 \\ \mathrm{H} & 0.44253900 & -1.77159500 & 0.00009600\end{array}$




$\begin{array}{lrrc}\mathrm{H} & -3.05297000 & 0.71696000 & -0.00009100 \\ \mathrm{H} & -1.61232700 & 2.74338800 & 0.00001000 \\ \mathrm{O} & -2.09364800 & -1.80011700 & -0.00009500 \\ \mathrm{H} & -3.03750300 & -1.57785300 & 0.00110000 \\ \mathrm{H} & 0.87387200 & 2.52357100 & 0.00012300 \\ \mathrm{O} & 2.18547200 & 0.35667500 & 0.00004900 \\ \mathrm{C} & 2.84169500 & -0.90073000 & -0.00002200 \\ \mathrm{H} & 3.91086000 & -0.68022000 & -0.00004900 \\ \mathrm{H} & 2.59223400 & -1.48684200 & 0.89441300 \\ \mathrm{H} & 2.59222100 & -1.48679100 & -0.89445400\end{array}$

\section{$11 p$}

$\begin{array}{lrrr}\mathrm{C} & 0.15253900 & 2.39244100 & -1.07733300 \\ \mathrm{C} & 0.80311700 & 1.17813400 & -0.86200300 \\ \mathrm{C} & 2.08018000 & 1.15827600 & -0.27769900 \\ \mathrm{C} & 2.69225600 & 2.37589500 & 0.06402400 \\ \mathrm{C} & 2.04262700 & 3.58546200 & -0.17148400 \\ \mathrm{C} & 0.76669800 & 3.59906900 & -0.73779200 \\ \mathrm{H} & -0.83902700 & 2.38723400 & -1.52060100 \\ \mathrm{H} & 0.31940400 & 0.25369800 & -1.15028300 \\ \mathrm{H} & 3.67716500 & 2.37948300 & 0.52447800 \\ \mathrm{H} & 2.53184500 & 4.51706800 & 0.09947700 \\ \mathrm{H} & 0.25633400 & 4.54172700 & -0.91399800 \\ \mathrm{C} & 2.78284000 & -0.12396600 & -0.04142600 \\ \mathrm{C} & 2.15232900 & -1.40028400 & 0.28657700 \\ \mathrm{O} & 0.94788700 & -1.56405300 & 0.43175300 \\ \mathrm{O} & 3.05344300 & -2.39458900 & 0.42631700 \\ \mathrm{C} & 2.51237900 & -3.68721100 & 0.75537900 \\ \mathrm{H} & 3.37710500 & -4.34450900 & 0.84411400 \\ \mathrm{H} & 1.96224900 & -3.64349500 & 1.69837900 \\ \mathrm{H} & 1.84494800 & -4.03500100 & -0.03702400 \\ \mathrm{~N} & 4.10117300 & -0.12813000 & -0.08332100 \\ \mathrm{~N} & 5.23742100 & -0.10886600 & -0.11893900 \\ \mathrm{C} & -4.68645300 & -0.64813300 & -1.28182800 \\ \mathrm{C} & -3.52767000 & -1.31398800 & -1.68428900 \\ \mathrm{C} & -2.38910200 & -1.23734900 & -0.87833200 \\ \mathrm{C} & -2.40556900 & -0.50009600 & 0.31637100 \\ \mathrm{C} & -3.57780000 & 0.16126900 & 0.69732400 \\ \mathrm{C} & -4.72743600 & 0.08832400 & -0.10170600 \\ \mathrm{H} & -5.57659400 & -0.70611900 & -1.90321200 \\ \mathrm{H} & -3.48901800 & -1.89053000 & -2.60253900 \\ \mathrm{O} & -1.26616000 & -1.88898400 & -1.29985000 \\ \mathrm{H} & -0.56792100 & -1.82031600 & -0.62008400\end{array}$




$\begin{array}{lrrr}\mathrm{H} & -5.62333700 & 0.60959800 & 0.21845000 \\ \mathrm{H} & -1.50581100 & -0.45355300 & 0.91870300 \\ \mathrm{O} & -3.69330700 & 0.90917100 & 1.83686900 \\ \mathrm{C} & -2.55095700 & 1.05072800 & 2.66400800 \\ \mathrm{H} & -2.85832700 & 1.69192100 & 3.49279200 \\ \mathrm{H} & -2.21509800 & 0.08330400 & 3.06125600 \\ \mathrm{H} & -1.71884000 & 1.52467200 & 2.12646300\end{array}$

\section{$10 q$}

$\begin{array}{lrrr}\mathrm{C} & -0.91200200 & -0.85678200 & 0.00014200 \\ \mathrm{C} & -2.24270000 & -0.44070700 & 0.00017900 \\ \mathrm{C} & -2.54793900 & 0.92659900 & -0.00010200 \\ \mathrm{C} & -1.51762100 & 1.86382400 & -0.00033800 \\ \mathrm{C} & -0.18154200 & 1.45998000 & -0.00034400 \\ \mathrm{C} & 0.11774700 & 0.09251400 & -0.00013100 \\ \mathrm{H} & -0.64882300 & -1.91137400 & 0.00025600 \\ \mathrm{H} & -1.76087700 & 2.92273800 & -0.00056900 \\ \mathrm{H} & 0.61958500 & 2.18920600 & -0.00051300 \\ \mathrm{C} & 1.51613800 & -0.42441800 & -0.00030400 \\ \mathrm{O} & 1.81370500 & -1.60380700 & -0.00106600 \\ \mathrm{O} & 2.43604400 & 0.56702600 & 0.00071600 \\ \mathrm{C} & 3.80385600 & 0.13204200 & 0.00050200 \\ \mathrm{H} & 4.40031200 & 1.04489400 & 0.00149400 \\ \mathrm{H} & 4.01644900 & -0.46800100 & 0.88936400 \\ \mathrm{H} & 4.01673000 & -0.46624400 & -0.88948100 \\ \mathrm{H} & -3.58991700 & 1.23076000 & -0.00016700 \\ \mathrm{O} & -3.29055700 & -1.31784300 & 0.00064000 \\ \mathrm{H} & -2.94261900 & -2.22329800 & -0.00032200\end{array}$

$11 q$

$\begin{array}{lrrr}\mathrm{C} & 3.66257100 & -2.43613800 & -0.40731300 \\ \mathrm{C} & 2.90582400 & -1.27418700 & -0.26376900 \\ \mathrm{C} & 3.54066400 & -0.02276200 & -0.20038500 \\ \mathrm{C} & 4.94212600 & 0.03102800 & -0.29871600 \\ \mathrm{C} & 5.68871300 & -1.13374700 & -0.45745000 \\ \mathrm{C} & 5.05283700 & -2.37535100 & -0.50831800 \\ \mathrm{H} & 3.15217700 & -3.39435600 & -0.44691300 \\ \mathrm{H} & 1.82865300 & -1.34719200 & -0.19944500 \\ \mathrm{H} & 5.45585000 & 0.98734200 & -0.24150000 \\ \mathrm{H} & 6.77090400 & -1.06867600 & -0.53074700 \\ \mathrm{H} & 5.63524100 & -3.28492900 & -0.62501100 \\ \mathrm{C} & 2.75769800 & 1.22566400 & -0.05484300\end{array}$




$\begin{array}{lrrr}\mathrm{C} & 1.45724100 & 1.37590000 & 0.60145300 \\ \mathrm{O} & 0.83272400 & 0.46513200 & 1.12999700 \\ \mathrm{O} & 1.02595400 & 2.64996500 & 0.57030400 \\ \mathrm{C} & -0.24580200 & 2.92165800 & 1.19857200 \\ \mathrm{H} & -0.32770400 & 4.00861000 & 1.20596000 \\ \mathrm{H} & -1.06601000 & 2.48411900 & 0.62418200 \\ \mathrm{H} & -0.25641400 & 2.52850000 & 2.21754200 \\ \mathrm{~N} & 3.28851600 & 2.34100400 & -0.51795300 \\ \mathrm{~N} & 3.76641800 & 3.29029000 & -0.92202200 \\ \mathrm{C} & -3.56643600 & -3.07286600 & -0.20699600 \\ \mathrm{C} & -2.26274700 & -3.17462800 & 0.27556400 \\ \mathrm{C} & -1.53767800 & -2.01395000 & 0.58080800 \\ \mathrm{C} & -2.13323700 & -0.76372900 & 0.39985300 \\ \mathrm{C} & -3.44101800 & -0.66985600 & -0.08801400 \\ \mathrm{C} & -4.16820300 & -1.82789800 & -0.39469900 \\ \mathrm{H} & -4.11968800 & -3.97852500 & -0.44071400 \\ \mathrm{H} & -1.78938300 & -4.14070000 & 0.42242800 \\ \mathrm{O} & -0.26769300 & -2.15743800 & 1.04692200 \\ \mathrm{H} & 0.12538200 & -1.27577300 & 1.20536900 \\ \mathrm{H} & -5.18057000 & -1.75058700 & -0.77272600 \\ \mathrm{H} & -1.58470800 & 0.13936400 & 0.63697700 \\ \mathrm{C} & -3.99974000 & 0.69802500 & -0.26616900 \\ \mathrm{O} & -3.39491200 & 1.73315600 & -0.04241800 \\ \mathrm{O} & -5.27567300 & 0.68668800 & -0.71357200 \\ \mathrm{C} & -5.87049100 & 1.97691400 & -0.91162700 \\ \mathrm{H} & -6.87998600 & 1.78025800 & -1.27418500 \\ \mathrm{H} & -5.90195000 & 2.53497400 & 0.02815600 \\ \mathrm{H} & -5.30346200 & 2.55404200 & -1.64711500\end{array}$

10r

$\begin{array}{lrrr}\mathrm{C} & -0.59093000 & -0.64049000 & -0.00001400 \\ \mathrm{C} & -0.41093700 & 0.75441600 & -0.00004700 \\ \mathrm{C} & 0.86722600 & 1.31760200 & -0.00010300 \\ \mathrm{C} & 1.99287300 & 0.49276100 & 0.00002200 \\ \mathrm{C} & 1.83967400 & -0.89249800 & 0.00004600 \\ \mathrm{C} & 0.55440000 & -1.44077700 & 0.00000200 \\ \mathrm{H} & 2.98421500 & 0.93790400 & 0.00003700 \\ \mathrm{H} & 2.71019000 & -1.54196300 & 0.00011400 \\ \mathrm{H} & 0.43083400 & -2.52164100 & 0.00003200 \\ \mathrm{H} & 0.97875300 & 2.40106500 & -0.00027300 \\ \mathrm{O} & -1.54837300 & 1.52131500 & -0.00027000 \\ \mathrm{H} & -1.29384700 & 2.45694800 & 0.00238400 \\ \mathrm{C} & -1.98128000 & -1.22294700 & 0.00003800\end{array}$




$\begin{array}{lrrr}\mathrm{H} & -2.55164400 & -0.89715400 & 0.87856000 \\ \mathrm{H} & -2.55175600 & -0.89717600 & -0.87840800 \\ \mathrm{H} & -1.94592300 & -2.31690300 & 0.00005000\end{array}$

$11 r$

$\begin{array}{llll}\text { C } & -0.30600100 & 2.64301000 & 0.53612300\end{array}$

$\begin{array}{llll}\text { C } & -0.80003700 & 1.34013200 & 0.49270400\end{array}$

$\begin{array}{llll}\text { C } & -2.13281700 & 1.10594300 & 0.11630700\end{array}$

$\begin{array}{llll}\text { C } & -2.95493000 & 2.20214200 & -0.19554100\end{array}$

C $\quad-2.45730300 \quad 3.50148400 \quad-0.13266800$

$\begin{array}{llll}\mathrm{C} & -1.12795400 & 3.72787900 & 0.22817200\end{array}$

$\begin{array}{llll}\mathrm{H} & 0.73046400 & 2.80384800 & 0.81843100\end{array}$

$\mathrm{H} \quad-0.15097900 \quad 0.51405700 \quad 0.75226000$

$\mathrm{H} \quad-3.98644800 \quad 2.04035400 \quad-0.49847800$

$\mathrm{H} \quad-3.10849000 \quad 4.33601700 \quad-0.37800000$

$\mathrm{H} \quad-0.73682600 \quad 4.74056100 \quad 0.26878100$

$\begin{array}{llll}\text { C } & -2.67603400 & -0.27116000 & 0.06471700\end{array}$

$\begin{array}{llll}\text { C } & -1.92665000 & -1.48476000 & -0.25316600\end{array}$

$\begin{array}{llll}\mathrm{O} & -0.73479700 & -1.51821700 & -0.52746900\end{array}$

$\begin{array}{llll}\mathrm{O} & -2.70577000 & -2.58678300 & -0.22245900\end{array}$

C $\quad-2.04470600 \quad-3.82773800 \quad-0.52856700$

$\mathrm{H} \quad-2.82326600 \quad-4.58814800 \quad-0.47035100$

$\mathrm{H} \quad-1.61213800 \quad-3.79439200 \quad-1.53137700$

$\mathrm{H} \quad-1.25486100 \quad-4.02998700 \quad 0.19919100$

$\begin{array}{llll}\mathrm{N} & -3.96740800 & -0.43674700 & 0.27673300\end{array}$

$\mathrm{N} \quad \quad-5.08338200 \quad-0.55608400 \quad 0.45893500$

$\begin{array}{llll}\mathrm{C} & 4.93500500 & 0.27351400 & 0.01886400\end{array}$

$\begin{array}{llll}\text { C } & 3.94045800 & -0.40442800 & 0.72758900\end{array}$

$\begin{array}{llll}\text { C } & 2.70372500 & -0.61556000 & 0.08808400\end{array}$

$\begin{array}{llll}\text { C } & 2.48040500 & -0.15508900 & -1.21388000\end{array}$

C $\quad 3.49454800 \quad 0.51833400 \quad-1.89685300$

C $\quad 4.72723200 \quad 0.73617000 \quad-1.28355700$

$\mathrm{H} \quad 5.89538800 \quad 0.44082500 \quad 0.50232400$

$\mathrm{H} \quad 3.31365200 \quad 0.87203800 \quad-2.90876000$

$\begin{array}{llll}\mathrm{O} & 1.74424800 & -1.27826800 & 0.80329300\end{array}$

$\mathrm{H} \quad 0.94942500 \quad-1.40784000 \quad 0.24993400$

$\mathrm{H} \quad \begin{array}{llll}5.52067100 & 1.25928300 & -1.80995800\end{array}$

$\mathrm{H} \quad 1.51345500 \quad-0.32477700 \quad-1.68043600$

C $\quad 4.15521100 \quad-0.90868600 \quad 2.13215300$

$\mathrm{H} \quad 3.43228900 \quad-0.46942000 \quad 2.83052700$

$\mathrm{H} \quad 4.01972700 \quad-1.99551000 \quad 2.19132600$

$\mathrm{H} \quad 5.16310600 \quad-0.66806300 \quad 2.48508800$ 


$\begin{array}{lrrr}\mathrm{C} & 0.45780600 & -0.42979100 & -0.00000500 \\ \mathrm{C} & -0.01444900 & 0.90252000 & 0.00001900 \\ \mathrm{C} & -1.39287200 & 1.14121200 & 0.00000600 \\ \mathrm{C} & -2.28111400 & 0.07157200 & 0.00000300 \\ \mathrm{C} & -1.82035300 & -1.25222900 & 0.00000600 \\ \mathrm{C} & -0.45500300 & -1.50056600 & -0.00000600 \\ \mathrm{H} & -3.34917700 & 0.27058800 & 0.00000900 \\ \mathrm{H} & -2.52381700 & -2.07850600 & -0.00000700 \\ \mathrm{H} & -0.07175000 & -2.51593900 & -0.00002600 \\ \mathrm{C} & 1.87076100 & -0.63974500 & -0.00005000 \\ \mathrm{~N} & 3.03318000 & -0.72267900 & 0.00004500 \\ \mathrm{H} & -1.73986400 & 2.16915200 & 0.00000300 \\ \mathrm{O} & 0.81518900 & 1.97273600 & 0.00000000 \\ \mathrm{H} & 1.74218300 & 1.67373600 & -0.00012600\end{array}$

$11 \mathrm{~s}$

$\begin{array}{lrrr}\mathrm{C} & -0.64192900 & 2.70433700 & 0.65112800 \\ \mathrm{C} & -1.04147400 & 1.37057100 & 0.58195100 \\ \mathrm{C} & -2.33722700 & 1.04600600 & 0.14775700 \\ \mathrm{C} & -3.21841600 & 2.08390600 & -0.19950400 \\ \mathrm{C} & -2.81535000 & 3.41438800 & -0.11330300 \\ \mathrm{C} & -1.52303200 & 3.73094300 & 0.30846800 \\ \mathrm{H} & 0.36494100 & 2.93724400 & 0.98572500 \\ \mathrm{H} & -0.34729500 & 0.59002800 & 0.86572400 \\ \mathrm{H} & -4.22224000 & 1.85221100 & -0.54674100 \\ \mathrm{H} & -3.51138200 & 4.20284400 & -0.38602100 \\ \mathrm{H} & -1.20629400 & 4.76813900 & 0.37071300 \\ \mathrm{C} & -2.78487800 & -0.36454300 & 0.07791000 \\ \mathrm{C} & -1.94979100 & -1.52338800 & -0.21966500 \\ \mathrm{O} & -0.75128900 & -1.47292900 & -0.47119700 \\ \mathrm{O} & -2.64735500 & -2.67625400 & -0.20203300 \\ \mathrm{C} & -1.89560800 & -3.87118500 & -0.48545300 \\ \mathrm{H} & -2.62204500 & -4.68215400 & -0.44081800 \\ \mathrm{H} & -1.44346200 & -3.81219100 & -1.47830500 \\ \mathrm{H} & -1.11211300 & -4.01564400 & 0.26247700 \\ \mathrm{~N} & -4.06755000 & -0.61733500 & 0.26077700 \\ \mathrm{~N} & -5.17630100 & -0.81208200 & 0.41617700 \\ \mathrm{C} & 4.83155100 & 0.45875000 & -0.41116500 \\ \mathrm{C} & 3.88424500 & -0.17249600 & 0.41350600 \\ \mathrm{C} & 2.60161800 & -0.47337500 & -0.09668500 \\ \mathrm{C} & 2.29276100 & -0.13187000 & -1.42065100\end{array}$




$\begin{array}{lrrr}\mathrm{C} & 3.24574200 & 0.49192500 & -2.22150800 \\ \mathrm{C} & 4.51853800 & 0.79065200 & -1.72437800 \\ \mathrm{H} & 5.81201700 & 0.68068900 & -0.00159500 \\ \mathrm{H} & 2.98939300 & 0.74948700 & -3.24576500 \\ \mathrm{O} & 1.72283900 & -1.07944300 & 0.73162500 \\ \mathrm{H} & 0.88579400 & -1.26742800 & 0.25782800 \\ \mathrm{H} & 5.25646400 & 1.27685000 & -2.35459800 \\ \mathrm{H} & 1.30233700 & -0.35635900 & -1.80525000 \\ \mathrm{C} & 4.21891500 & -0.50844300 & 1.76349500 \\ \mathrm{~N} & 4.52189900 & -0.76655100 & 2.85705200\end{array}$

10j

$\begin{array}{lrrr}\mathrm{C} & -0.42754200 & 0.99786000 & -0.00003300 \\ \mathrm{C} & -1.83452600 & 1.01326800 & 0.00004600 \\ \mathrm{C} & -2.55560500 & -0.16892400 & 0.00006200 \\ \mathrm{C} & -1.90500600 & -1.41569300 & 0.00000200 \\ \mathrm{C} & -0.52326000 & -1.45913600 & -0.00006700 \\ \mathrm{C} & 0.21649900 & -0.26561200 & -0.00008500 \\ \mathrm{H} & -2.32455000 & 1.98128700 & 0.00008600 \\ \mathrm{H} & -2.47865600 & -2.33652800 & 0.00001400 \\ \mathrm{H} & 0.01909200 & -2.39660900 & -0.00010300 \\ \mathrm{~N} & 1.66046400 & -0.36204300 & -0.00016700 \\ \mathrm{O} & 2.32641400 & 0.69974300 & 0.00010300 \\ \mathrm{O} & 2.18764700 & -1.46868900 & 0.00012500 \\ \mathrm{H} & -3.64122800 & -0.12767700 & 0.00010200 \\ \mathrm{O} & 0.21095000 & 2.17312500 & -0.00001000 \\ \mathrm{H} & 1.17864400 & 1.96981100 & -0.00022200\end{array}$

11j

$\begin{array}{llrr}\mathrm{C} & 4.90437000 & -2.23012300 & -0.26058300 \\ \mathrm{C} & 4.55724000 & -0.88240600 & -0.29289200 \\ \mathrm{C} & 3.23029700 & -0.47519500 & -0.06064300 \\ \mathrm{C} & 2.26035800 & -1.45756100 & 0.20744800 \\ \mathrm{C} & 2.62161800 & -2.80397400 & 0.24149100 \\ \mathrm{C} & 3.93817000 & -3.20121000 & 0.00801300 \\ \mathrm{H} & 5.93634200 & -2.51853700 & -0.44213200 \\ \mathrm{H} & 5.33027700 & -0.14576200 & -0.49541700 \\ \mathrm{H} & 1.23405600 & -1.17240300 & 0.39352300 \\ \mathrm{H} & 1.85472100 & -3.54500900 & 0.45014400 \\ \mathrm{H} & 4.20953000 & -4.25289700 & 0.03466500 \\ \mathrm{C} & 2.87088600 & 0.96012500 & -0.11369700 \\ \mathrm{C} & 1.61481300 & 1.57294300 & 0.33593600\end{array}$




$\begin{array}{lrrr}\mathrm{O} & 0.68314800 & 0.96759000 & 0.83634900 \\ \mathrm{O} & 1.61167300 & 2.91152000 & 0.13052600 \\ \mathrm{C} & 0.42799900 & 3.60211400 & 0.56962000 \\ \mathrm{H} & 0.58223100 & 4.64257800 & 0.28263300 \\ \mathrm{H} & -0.46165800 & 3.19071300 & 0.08852500 \\ \mathrm{H} & 0.31825800 & 3.51674400 & 1.65417800 \\ \mathrm{~N} & 3.76105900 & 1.80338700 & -0.59642800 \\ \mathrm{~N} & 4.54806200 & 2.51037800 & -1.01583500 \\ \mathrm{C} & -3.31583400 & 0.62254200 & 0.07311100 \\ \mathrm{C} & -4.42925700 & 1.44149700 & -0.19777700 \\ \mathrm{C} & -5.64995900 & 0.89485900 & -0.55559300 \\ \mathrm{C} & -5.81486900 & -0.49685100 & -0.66047800 \\ \mathrm{C} & -4.73880300 & -1.32516400 & -0.40122700 \\ \mathrm{C} & -3.49729300 & -0.77920300 & -0.03693500 \\ \mathrm{H} & -4.28991000 & 2.51424100 & -0.11343200 \\ \mathrm{H} & -6.77378100 & -0.91998200 & -0.94082300 \\ \mathrm{H} & -4.81889700 & -2.40308500 & -0.46946100 \\ \mathrm{~N} & -2.40831500 & -1.69771100 & 0.22273400 \\ \mathrm{O} & -1.31132900 & -1.21738000 & 0.57223100 \\ \mathrm{O} & -2.59793300 & -2.90386400 & 0.09270400 \\ \mathrm{H} & -6.48892500 & 1.55535900 & -0.75688700 \\ \mathrm{O} & -2.17941200 & 1.24090500 & 0.40911300 \\ \mathrm{H} & -1.46546700 & 0.58114300 & 0.57903000\end{array}$

$10 t$

$\begin{array}{lrrr}\text { C } & -0.48691600 & 0.69655200 & -0.00001100 \\ \mathrm{C} & -0.54839700 & -0.69968500 & 0.00001000 \\ \mathrm{C} & 0.64897000 & -1.41658400 & 0.00002100 \\ \mathrm{C} & 1.86670100 & -0.73355700 & 0.00001100 \\ \mathrm{C} & 1.90437000 & 0.66282300 & -0.00001300 \\ \mathrm{C} & 0.71048800 & 1.39012300 & -0.00002100 \\ \mathrm{H} & 2.79340000 & -1.30006000 & 0.00002000 \\ \mathrm{H} & 2.85465900 & 1.18751000 & -0.00002200 \\ \mathrm{H} & 0.69530100 & 2.47534100 & -0.00003700 \\ \mathrm{H} & 0.60431300 & -2.50110500 & 0.00003900 \\ \mathrm{O} & -1.74534100 & -1.35200400 & 0.00001900 \\ \mathrm{H} & -2.44640900 & -0.67875800 & 0.00003800 \\ \mathrm{~F} & -1.67886900 & 1.35945300 & -0.00001900\end{array}$

$11 t$

$\begin{array}{llll}\mathrm{C} & 2.09587200 & 2.88928300 & -0.44823000 \\ \mathrm{C} & 1.67406800 & 1.56070200 & -0.41916800\end{array}$ 


$\begin{array}{lrrr}\mathrm{C} & 2.53472500 & 0.56006900 & 0.06475200 \\ \mathrm{C} & 3.81322800 & 0.92978400 & 0.51939100 \\ \mathrm{C} & 4.22039900 & 2.26095500 & 0.49456800 \\ \mathrm{C} & 3.36412500 & 3.24972900 & 0.00769000 \\ \mathrm{H} & 1.41670900 & 3.64796800 & -0.82740700 \\ \mathrm{H} & 0.68445300 & 1.30581100 & -0.77355000 \\ \mathrm{H} & 4.50138900 & 0.17386800 & 0.88886500 \\ \mathrm{H} & 5.21349100 & 2.52173500 & 0.85029800 \\ \mathrm{H} & 3.68217500 & 4.28821300 & -0.01561800 \\ \mathrm{C} & 2.10863500 & -0.85689000 & 0.11974300 \\ \mathrm{C} & 1.04403700 & -1.48321200 & -0.66510300 \\ \mathrm{O} & 0.35371100 & -0.89866400 & -1.48823800 \\ \mathrm{O} & 0.92012500 & -2.79757900 & -0.39196200 \\ \mathrm{C} & -0.12375900 & -3.48947700 & -1.10353700 \\ \mathrm{H} & -0.04538100 & -4.52845600 & -0.78346000 \\ \mathrm{H} & -1.09650100 & -3.07390500 & -0.83508700 \\ \mathrm{H} & 0.02905700 & -3.40597400 & -2.18220500 \\ \mathrm{~N} & 2.76217100 & -1.66793700 & 0.92889600 \\ \mathrm{~N} & 3.34047600 & -2.34928900 & 1.63205000 \\ \mathrm{C} & -4.89947000 & 1.58831000 & 0.38351300 \\ \mathrm{C} & -3.89997900 & 1.61430100 & -0.58870300 \\ \mathrm{C} & -2.85817600 & 0.67966100 & -0.56539900 \\ \mathrm{C} & -2.86409100 & -0.26774000 & 0.46543800 \\ \mathrm{C} & -3.84946600 & -0.31052000 & 1.43781800 \\ \mathrm{C} & -4.88145400 & 0.63006400 & 1.39963500 \\ \mathrm{H} & -5.69805600 & 2.32377300 & 0.34474400 \\ \mathrm{H} & -3.90284000 & 2.35263000 & -1.38460400 \\ \mathrm{H} & -3.79360800 & -1.07273800 & 2.20876300 \\ \mathrm{O} & -1.90215900 & 0.73158900 & -1.52398700 \\ \mathrm{H} & -1.23788400 & 0.01911100 & -1.41344700 \\ \mathrm{H} & -5.66006300 & 0.60994800 & 2.15581600 \\ \mathrm{~F} & -1.85078200 & -1.18380800 & 0.49548000 \\ & & & \\ & & & \end{array}$

$10 u$

$\begin{array}{lrrc}\mathrm{C} & 0.38845000 & -0.35668400 & -0.00000800 \\ \mathrm{C} & -0.12696300 & 0.94845000 & 0.00000700 \\ \mathrm{C} & -1.51753600 & 1.11400400 & 0.00002900 \\ \mathrm{C} & -2.35889900 & 0.00583100 & 0.00001000 \\ \mathrm{C} & -1.83120500 & -1.28856200 & -0.00001000 \\ \mathrm{C} & -0.44914100 & -1.46915400 & -0.00002100 \\ \mathrm{H} & -3.43495500 & 0.15430100 & 0.00003200 \\ \mathrm{H} & -2.48747000 & -2.15330100 & -0.00004000 \\ \mathrm{H} & -0.01355100 & -2.46284800 & -0.00003800\end{array}$




$\begin{array}{lrrr}\mathrm{H} & -1.90958300 & 2.12608900 & 0.00007000 \\ \mathrm{O} & 0.65084700 & 2.06066600 & -0.00004900 \\ \mathrm{H} & 1.58539800 & 1.78993900 & 0.00006800 \\ \mathrm{Cl} & 2.14265600 & -0.56840200 & 0.00001500\end{array}$

$11 u$

$\begin{array}{lrrr}\mathrm{C} & -0.66398900 & -2.68654600 & -0.59005900 \\ \mathrm{C} & -1.09302100 & -1.36121300 & -0.53589200 \\ \mathrm{C} & -2.40865800 & -1.06359000 & -0.14422100 \\ \mathrm{C} & -3.27922400 & -2.11954400 & 0.17480700 \\ \mathrm{C} & -2.84617000 & -3.44138000 & 0.10300300 \\ \mathrm{C} & -1.53424700 & -3.73113600 & -0.27546600 \\ \mathrm{H} & 0.35872600 & -2.89770200 & -0.88901000 \\ \mathrm{H} & -0.40559000 & -0.56735600 & -0.79784700 \\ \mathrm{H} & -4.29829300 & -1.90871000 & 0.48945600 \\ \mathrm{H} & -3.53428400 & -4.24398500 & 0.35385400 \\ \mathrm{H} & -1.19391700 & -4.76156100 & -0.32519100 \\ \mathrm{C} & -2.88682200 & 0.33751100 & -0.08748100 \\ \mathrm{C} & -2.08255700 & 1.51460300 & 0.22809700 \\ \mathrm{O} & -0.88937000 & 1.49200000 & 0.50246000 \\ \mathrm{O} & -2.80714600 & 2.65180700 & 0.19734000 \\ \mathrm{C} & -2.08809600 & 3.86159800 & 0.49977100 \\ \mathrm{H} & -2.83084200 & 4.65694800 & 0.44215100 \\ \mathrm{H} & -1.65535600 & 3.80952700 & 1.50166100 \\ \mathrm{H} & -1.29194500 & 4.02556900 & -0.23063200 \\ \mathrm{~N} & -4.16988500 & 0.56315100 & -0.29766600 \\ \mathrm{~N} & -5.27918700 & 0.73431600 & -0.47735700 \\ \mathrm{C} & 4.71174100 & -0.46454300 & 0.47546400 \\ \mathrm{C} & 3.77403700 & 0.16611800 & -0.33860700 \\ \mathrm{C} & 2.49428900 & 0.48411400 & 0.14613200 \\ \mathrm{C} & 2.17981500 & 0.14618400 & 1.47082700 \\ \mathrm{C} & 3.11921300 & -0.48376400 & 2.28478200 \\ \mathrm{C} & 4.38807400 & -0.79197600 & 1.79286200 \\ \mathrm{H} & 5.69150200 & -0.69382200 & 0.06892800 \\ \mathrm{H} & 2.85315100 & -0.73546600 & 3.30781700 \\ \mathrm{O} & 1.61553100 & 1.09673000 & -0.68477100 \\ \mathrm{H} & 0.78580300 & 1.28798100 & -0.20270600 \\ \mathrm{H} & 5.12312500 & -1.28251400 & 2.42381200 \\ \mathrm{H} & 1.18793100 & 0.38144500 & 1.84673300 \\ \mathrm{Cl} & 4.19382800 & 0.57202700 & -1.99460100\end{array}$

$10 v$ 


$\begin{array}{lrrr}\mathrm{C} & 0.43989100 & -0.08353400 & 0.00004300 \\ \mathrm{C} & -0.47417500 & 0.99045500 & 0.00000100 \\ \mathrm{C} & -1.84062100 & 0.73338500 & -0.00008500 \\ \mathrm{C} & -2.30231300 & -0.58712900 & -0.00004100 \\ \mathrm{C} & -1.39920800 & -1.64663000 & 0.00003400 \\ \mathrm{C} & -0.01998200 & -1.39697200 & 0.00004100 \\ \mathrm{H} & -3.37129900 & -0.77985200 & -0.00003600 \\ \mathrm{H} & -1.75450500 & -2.67289400 & 0.00011000 \\ \mathrm{H} & 0.67941500 & -2.22605400 & 0.00002000 \\ \mathrm{H} & -2.52613500 & 1.57521100 & -0.00018800 \\ \mathrm{O} & -0.01672900 & 2.27526900 & 0.00015600 \\ \mathrm{H} & 0.95529400 & 2.22733500 & -0.00067200 \\ \mathrm{O} & 1.75761100 & 0.31632900 & -0.00002000 \\ \mathrm{C} & 2.76095000 & -0.68631900 & -0.00003600 \\ \mathrm{H} & 3.71689500 & -0.15944200 & 0.00000900 \\ \mathrm{H} & 2.69299200 & -1.31830800 & 0.89491800 \\ \mathrm{H} & 2.69303700 & -1.31830800 & -0.89498900\end{array}$

$11 v$

$\begin{array}{lrrr}\mathrm{C} & 2.09637400 & -2.98428300 & 0.35714300 \\ \mathrm{C} & 1.71258000 & -1.64515300 & 0.41197700 \\ \mathrm{C} & 2.58940300 & -0.64505100 & -0.04329500 \\ \mathrm{C} & 3.84286700 & -1.02285500 & -0.55574500 \\ \mathrm{C} & 4.21140300 & -2.36435100 & -0.61512000 \\ \mathrm{C} & 3.34035600 & -3.35349400 & -0.15594000 \\ \mathrm{H} & 1.40684400 & -3.74389600 & 0.71499600 \\ \mathrm{H} & 0.74098800 & -1.38157700 & 0.80890400 \\ \mathrm{H} & 4.54086000 & -0.26567100 & -0.90396800 \\ \mathrm{H} & 5.18578400 & -2.63342100 & -1.01384300 \\ \mathrm{H} & 3.62850900 & -4.40012500 & -0.19783600 \\ \mathrm{C} & 2.19820600 & 0.78269500 & -0.01211900 \\ \mathrm{C} & 1.19798900 & 1.38665600 & 0.86990500 \\ \mathrm{O} & 0.57968600 & 0.78327200 & 1.73167900 \\ \mathrm{O} & 1.03532600 & 2.70845900 & 0.62599100 \\ \mathrm{C} & 0.07031900 & 3.37270400 & 1.46503900 \\ \mathrm{H} & 0.10210000 & 4.41967400 & 1.16297700 \\ \mathrm{H} & -0.92324000 & 2.95001100 & 1.30010900 \\ \mathrm{H} & 0.34288700 & 3.26685600 & 2.51764800 \\ \mathrm{~N} & 2.81685100 & 1.61520100 & -0.82486300 \\ \mathrm{~N} & 3.36482300 & 2.31837400 & -1.53231400 \\ \mathrm{C} & -4.84001200 & -1.72567600 & -0.12218100 \\ \mathrm{C} & -3.82921800 & -1.64503100 & 0.83832200 \\ \mathrm{C} & -2.78692900 & -0.72984600 & 0.69823200\end{array}$




$\begin{array}{lrrr}\mathrm{C} & -2.76519200 & 0.11986700 & -0.42879500 \\ \mathrm{C} & -3.77496000 & 0.03781600 & -1.38643800 \\ \mathrm{C} & -4.81436400 & -0.88752100 & -1.23348500 \\ \mathrm{H} & -5.64354900 & -2.44591700 & 0.00346300 \\ \mathrm{H} & -3.82526700 & -2.28882600 & 1.71263700 \\ \mathrm{H} & -3.76019900 & 0.68825500 & -2.25402900 \\ \mathrm{O} & -1.82139900 & -0.69540100 & 1.65371000 \\ \mathrm{H} & -1.15781400 & -0.00137800 & 1.47135100 \\ \mathrm{H} & -5.59508000 & -0.94271600 & -1.98651000 \\ \mathrm{O} & -1.69836500 & 0.99249600 & -0.47575500 \\ \mathrm{C} & -1.54833100 & 1.79034600 & -1.64049000 \\ \mathrm{H} & -2.39328200 & 2.47993900 & -1.76794400 \\ \mathrm{H} & -0.63163300 & 2.36407900 & -1.49487900 \\ \mathrm{H} & -1.45455600 & 1.16812400 & -2.53988800\end{array}$

10w

$\begin{array}{lrrr}\mathrm{C} & -1.09698200 & 0.93313100 & -0.00000300 \\ \mathrm{C} & -2.47115400 & 0.64178200 & -0.00000600 \\ \mathrm{C} & -2.90599200 & -0.67514600 & 0.00000400 \\ \mathrm{C} & -1.98918000 & -1.73853500 & 0.00000000 \\ \mathrm{C} & -0.63127300 & -1.46294200 & -0.00000400 \\ \mathrm{C} & -0.16148400 & -0.13499200 & -0.00001400 \\ \mathrm{H} & -2.33960700 & -2.76595100 & 0.00000000 \\ \mathrm{H} & 0.09480200 & -2.26835100 & -0.00000900 \\ \mathrm{C} & 1.27390700 & 0.18136900 & -0.00001300 \\ \mathrm{O} & 1.73070400 & 1.32668700 & -0.00005800 \\ \mathrm{O} & 2.07486600 & -0.89824600 & 0.00000300 \\ \mathrm{C} & 3.48710900 & -0.62984700 & 0.00002900 \\ \mathrm{H} & 3.96615600 & -1.60908600 & 0.00004300 \\ \mathrm{H} & 3.76960600 & -0.06228000 & -0.89020000 \\ \mathrm{H} & 3.76956600 & -0.06227200 & 0.89026500 \\ \mathrm{H} & -3.97308100 & -0.88229100 & 0.00000300 \\ \mathrm{H} & -3.16898100 & 1.47286000 & 0.00000500 \\ \mathrm{O} & -0.73127100 & 2.22640500 & 0.00003300 \\ \mathrm{H} & 0.25743500 & 2.24968100 & 0.00010600\end{array}$

\section{$11 w$}

$\begin{array}{llll}\text { C } & -4.61604200 & 1.70877700 & -0.89273000 \\ C & -4.03552800 & 0.44698000 & -0.98879200 \\ \text { C } & -2.95681600 & 0.08371100 & -0.16092500 \\ \text { C } & -2.47537200 & 1.02386500 & 0.76870600 \\ C & -3.07142100 & 2.28178900 & 0.86060100\end{array}$




\begin{tabular}{lrrr}
$\mathrm{C}$ & -4.13954900 & 2.63592900 & 0.03536400 \\
$\mathrm{H}$ & -5.44885500 & 1.96237800 & -1.54325600 \\
$\mathrm{H}$ & -4.43360100 & -0.25975800 & -1.71206000 \\
$\mathrm{H}$ & -1.64342200 & 0.76706200 & 1.40998600 \\
$\mathrm{H}$ & -2.69135400 & 2.98967400 & 1.59302800 \\
$\mathrm{H}$ & -4.59675000 & 3.61830100 & 0.11520700 \\
$\mathrm{C}$ & -2.34184500 & -1.25612000 & -0.27750000 \\
$\mathrm{C}$ & -1.37688700 & -1.86835600 & 0.65107000 \\
$\mathrm{O}$ & -0.94427900 & -1.33877300 & 1.65758200 \\
$\mathrm{O}$ & -1.02727300 & -3.11192200 & 0.24707400 \\
$\mathrm{C}$ & -0.07699500 & -3.78898900 & 1.09227400 \\
$\mathrm{H}$ & 0.09262400 & -4.75332100 & 0.61222500 \\
$\mathrm{H}$ & 0.84863400 & -3.21362700 & 1.15784500 \\
$\mathrm{H}$ & -0.49200800 & -3.92850200 & 2.09410600 \\
$\mathrm{~N}$ & -2.69579800 & -2.01121700 & -1.29669100 \\
$\mathrm{~N}$ & -3.01695700 & -2.64562700 & -2.18565100 \\
$\mathrm{C}$ & 3.06324100 & -0.58348300 & 0.32470200 \\
$\mathrm{C}$ & 4.11285300 & -1.39666200 & -0.13343300 \\
$\mathrm{C}$ & 5.05872200 & -0.89101500 & -1.01310600 \\
$\mathrm{C}$ & 4.98698700 & 0.43628100 & -1.46272900 \\
$\mathrm{C}$ & 3.95540500 & 1.24763400 & -1.01716900 \\
$\mathrm{C}$ & 2.98295100 & 0.76064700 & -0.12254300 \\
$\mathrm{H}$ & 4.15765300 & -2.42130000 & 0.22161900 \\
$\mathrm{H}$ & 5.73095000 & 0.82440100 & -2.15142700 \\
$\mathrm{H}$ & 3.88028500 & 2.27662200 & -1.35075300 \\
$\mathrm{O}$ & 1.03542900 & 1.23782900 & 1.17553600 \\
$\mathrm{H}$ & 5.86420200 & -1.53541900 & -1.35593100 \\
$\mathrm{O}$ & 2.17657300 & -1.13616700 & 1.17315700 \\
$\mathrm{H}$ & 1.49466100 & -0.45846100 & 1.40592500 \\
$\mathrm{C}$ & 1.88393100 & 1.60903800 & 0.36491200 \\
$\mathrm{H}$ & 1.87750700 & 2.84866400 & -0.15497900 \\
$\mathrm{H}$ & 0.81358100 & 3.70797400 & 0.29077600 \\
& 0.88097200 & 3.87012800 & 1.36981200 \\
$\mathrm{H}$ & 0.95715500 & 4.64517200 & -0.24739100 \\
\hline & -0.15883100 & 3.26950800 & 0.05363900
\end{tabular}




\section{References}

[1] Liptak, M. D.; Gross, K. C.; Seybold, P. G.; Feldgus, S.; Shields, G. C. Absolute $\mathrm{p} K_{\mathrm{a}}$ Determinations for Substituted Phenols. J. Am. Chem. Soc. 2002, 124, 6421-6427.

[2] Zhang, Z.; Yu, Y.; Huang, F.; Yi, X.; Xu, Y.; He, Y.; Baell, J. B.; He, H. Catalytic O-H bond insertion reactions using surface modified sewage sludge as catalyst. Green Chem. 2020, 22, 1594-1604.

[3] Gaussian 09, Revision D.01, M. J. Frisch, G. W. Trucks, H. B. Schlegel, G. E. Scuseria, M. A. Robb, Cheeseman, J. R.; Scalmani, G.; Barone, V.; Mennucci, B.; Petersson, G. A.; Nakatsuji, H.; Caricato, M.; Li, X.; Hratchian, H. P.; Izmaylov, A. F.; Bloino, J.; Zheng, G.; Sonnenberg, J. K.; Hada, M.; Ehara, M.; Toyota, K.; Fukuda, R.; Hasegawa, J.; Ishida, M.; Nakajima, T.; Honda, Y.; Kitao, O.; Nakai, H.; Vreven, T.; Montgomery, J. A.; Peralta, Jr. J. E.; Ogliaro, F.; Bearpark, M.; Heyd, J. J.; Brothers, E.; Kudin, K. N.; Staroverov, V. N.; Kobayashi, R.; Normand, J.; Raghavachari, K.; Rendell, A.; Burant, J. C.; Iyengar, S. S.; Tomasi, J.; Cossi, M.; Rega, N.; Millam, J. M.; Klene, M.; Knox, J. E.; Cross, J. B.; Bakken, V.; Adamo, C.; Jaramillo, J.; Gomperts, R.; Stratmann, R. E.; Yazyev, O.; Austin, A. J.; Cammi, R.; Pomelli, C.; Ochterski, J. W.; Martin, R. L.; Morokuma, K.; Zakrzewski, V. G.; Voth, G. A.; Salvador, P.; Dannenberg, J. J.; Dapprich, S.; Daniels, A. D.; Farkas, Ö.; Foresman, J. B.; Ortiz, J. V.; Cioslowski, J.; Fox, D. J. Gaussian, Inc., Wallingford CT, 2013.

[4] (a) Becke, A.D.; Density-Functional Thermochemistry. III. The role of Exact Exchange. J. Chem. Phys. 1993, 98, 5648-5652. (b) Lee, L.; Yang, W.; Parr, R. G.; Development of the Colle-Salvetti Correlation-Energy Formula into a Functional of the Electron Density. Phys. Rev. B: Condens. Matter Mater. Phys. 1988, 37, 785-789.

[5] Hehre, W. J.; Radom, L.; Schleyer, P. v. R.; Pople, J. A. Ab Initio Molecular Orbital Theory; Wiley: New York, 1986.

[6] Moller, C.; Plesset, M. S.; Note on an Approximation Treatment for Many-Electron Systems. Phys. Rev. 1934, 46, 618-622.

[7] (a) McLean, A. D.; Chandler, G. S. Contracted Gaussian-basis sets for molecular calculations. 1. 2nd row atoms, $Z=11-18$. J. Chem. Phys. 1980, 72, 5639-5648. (b) Raghavachari, K.; Binkley, J. S.; Seeger, R.; Pople, J. A. Self-Consistent Molecular Orbital Methods. 20. Basis set for correlated wave-functions. J. Chem. Phys. 1980, 72, 650-654.

[8] Simon, S.; Duran, M.; Dannenberg, J. J. How does basis set superposition error change the potential surfaces for hydrogen bonded dimers? J. Chem. Phys. 1996, 105, 11024-11031.

[9] Marenich, A. V.; Cramer, C. J.; Truhlar, D. G. Universal Solvation Model Based on Solute Electron Density and on a Continuum Model of the Solvent Defined by the Bulk Dielectric Constant and Atomic Surface Tensions. J. Phys. Chem. B 2009, 113, 6378-6396.

[10] Legault, C. Y.; CYLview, 1.0b, Universitéde Sherbrooke, 2009. http://www.cylview.org. 


\section{Spectra}

Methyl 2-(4-cyanophenoxy)-2-phenylacetate (12a)

${ }^{1}$ H NMR (400 MHz, Chloroform- $d$ )

${ }_{\mathrm{NC}}^{\mathrm{O}} \mathrm{Y}_{\mathrm{Ph}}^{\mathrm{CO}_{2} \mathrm{Me}}$

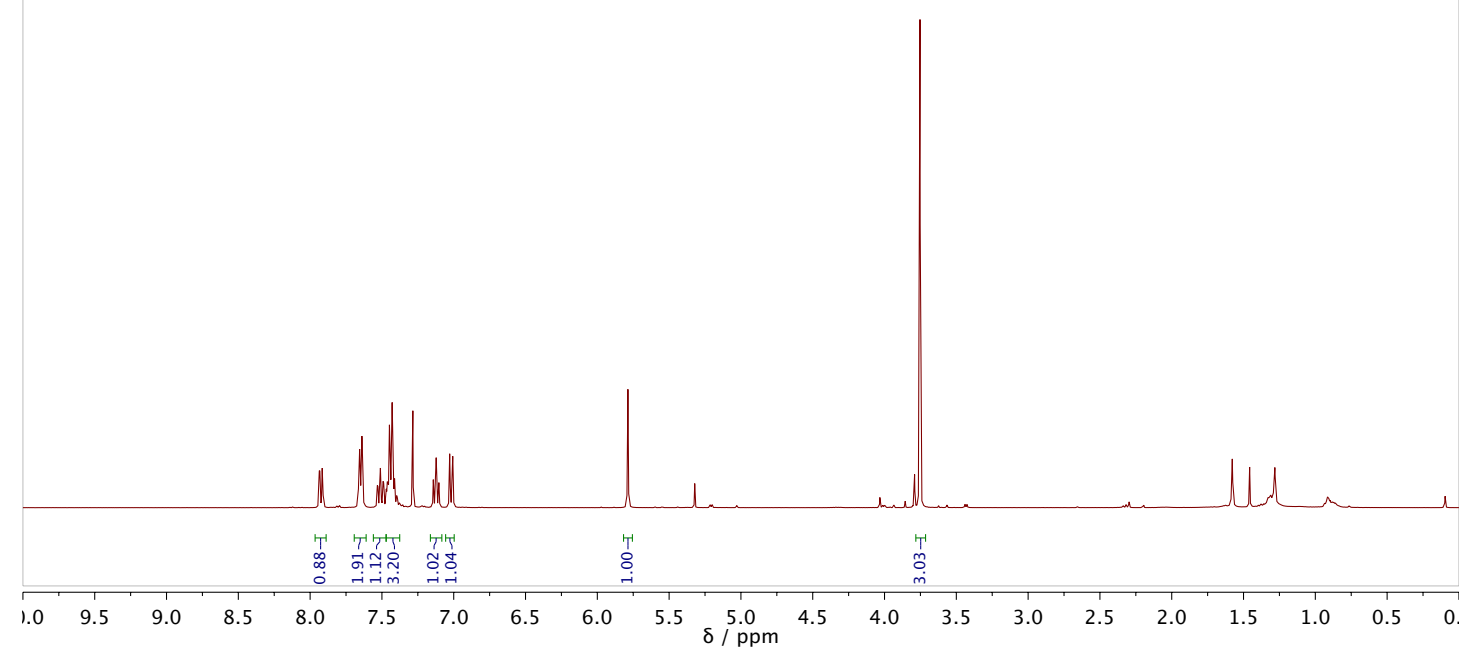

${ }^{13}$ C NMR (101MHz, Chloroform- $d$ )

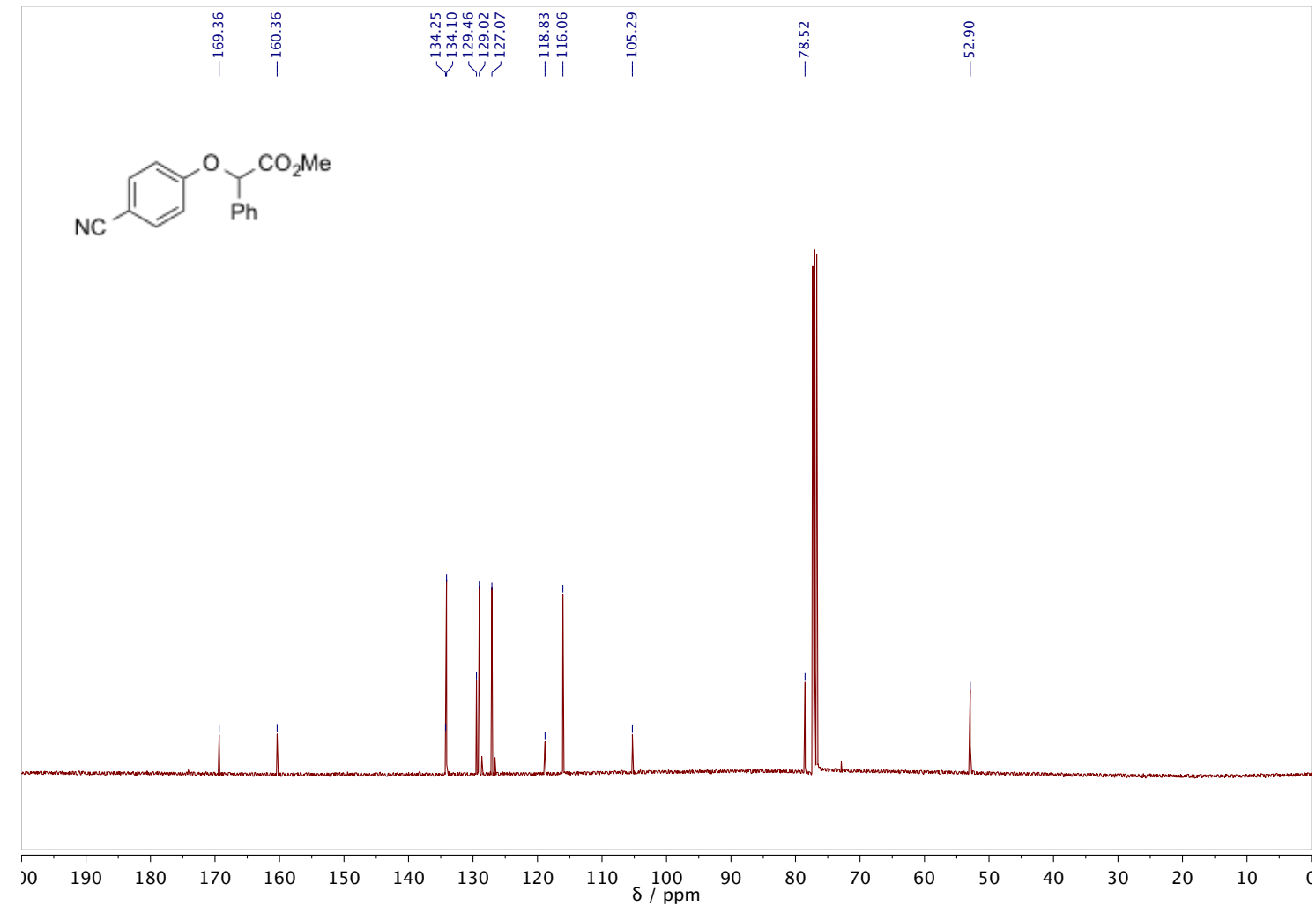


Ethyl 2-(4-cyanophenoxy)-2-phenylacetate (12b)

${ }^{1}$ H NMR (600 MHz, Chloroform- $d$ )
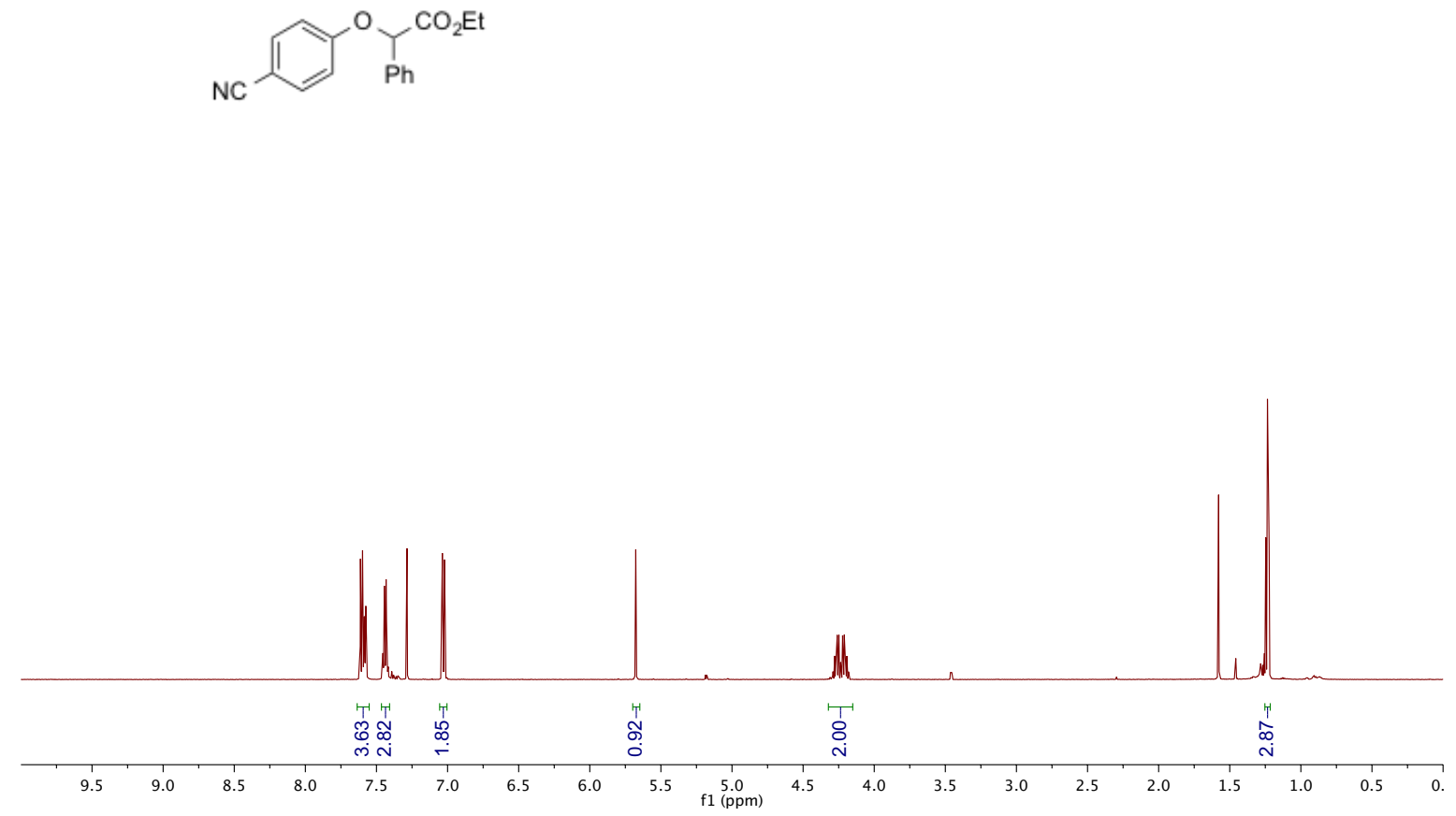

${ }^{13}$ C NMR (151 MHz, Chloroform- $d$ )
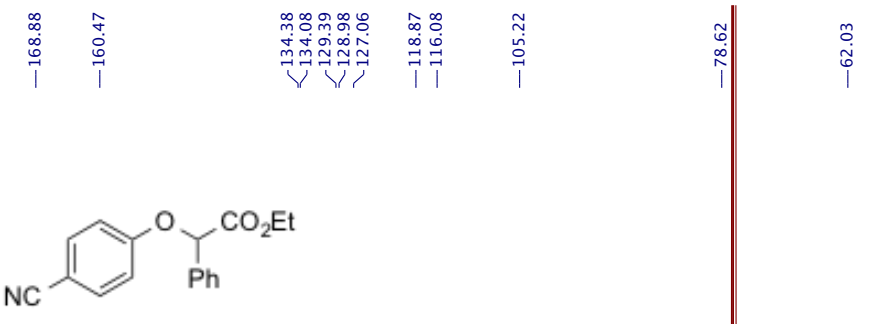

$\underset{\substack{0 \\ \dot{0}}}{\stackrel{1}{\dot{1}}}$

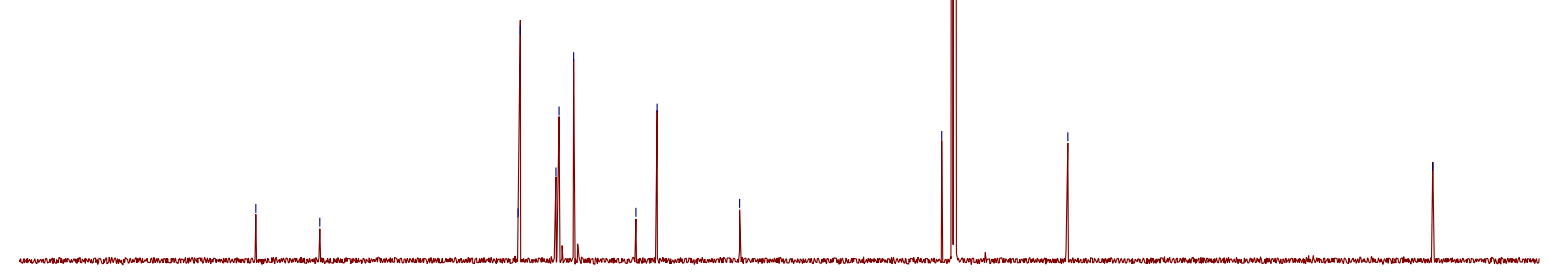

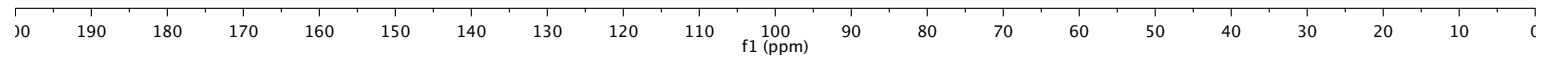


Allyl 2-(4-cyanophenoxy)-2-phenylacetate (12c)

${ }^{1}$ H NMR (600 MHz, Chloroform-d)<smiles>N#Cc1ccc(OC(C(=O)OCc2ccccc2)c2ccccc2)cc1</smiles>

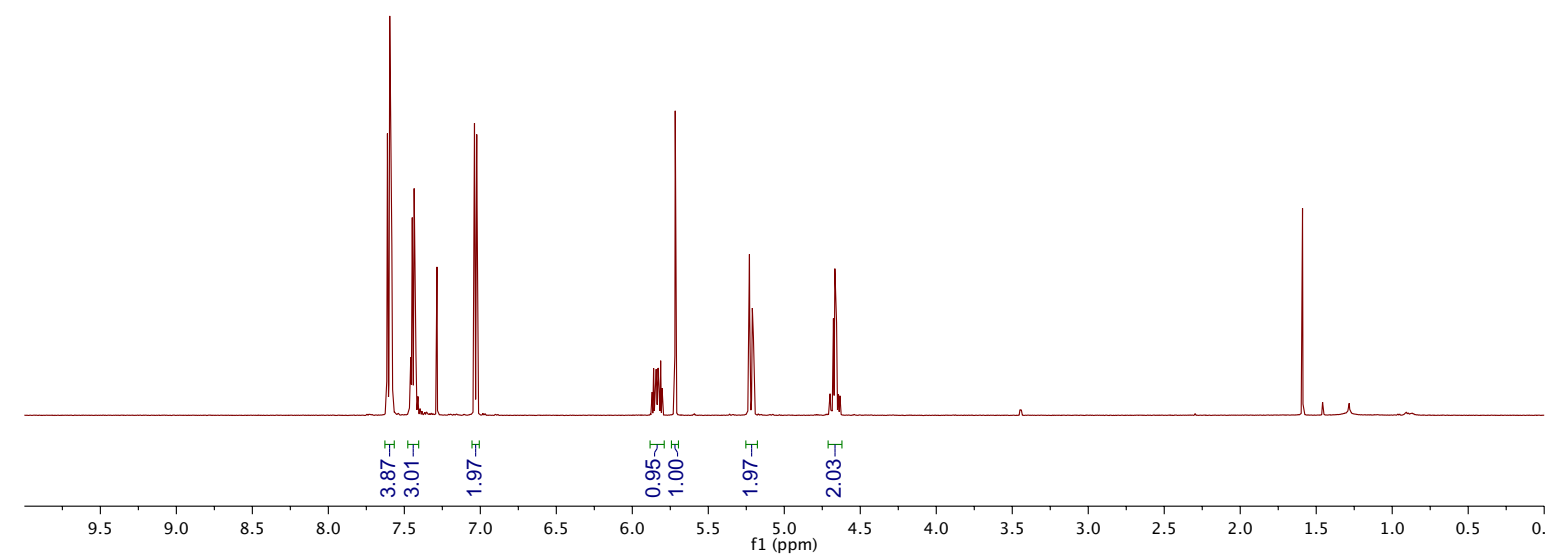

${ }^{13}$ C NMR (151 MHz, Chloroform- $d$ )

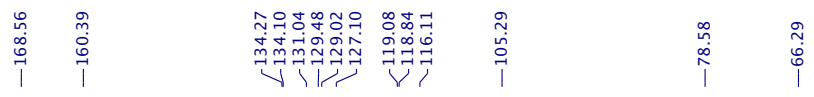<smiles>N#Cc1ccc(OC(C(=O)OCc2ccccc2)c2ccccc2)cc1</smiles>

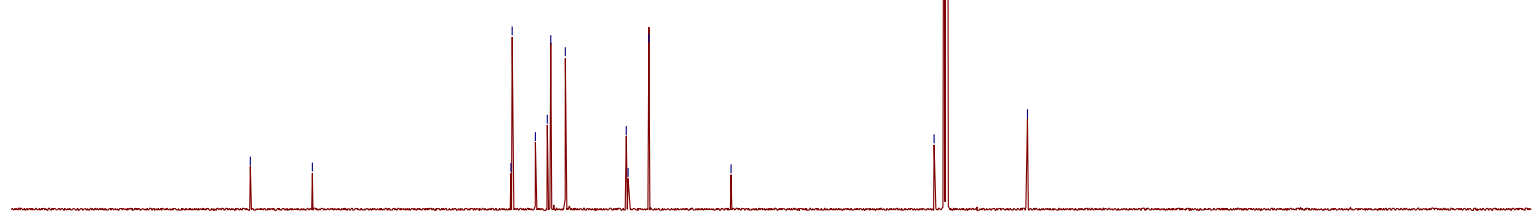

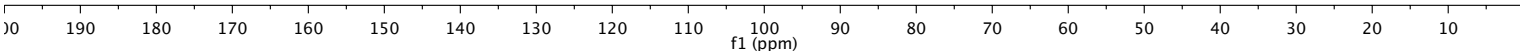


Benzyl 2-(4-cyanophenoxy)-2-phenylacetate (12d)

${ }^{1}$ H NMR (600 MHz, Chloroform- $d$ )<smiles>N#Cc1ccc(OC(C(=O)OCc2ccccc2)c2ccccc2)cc1</smiles>

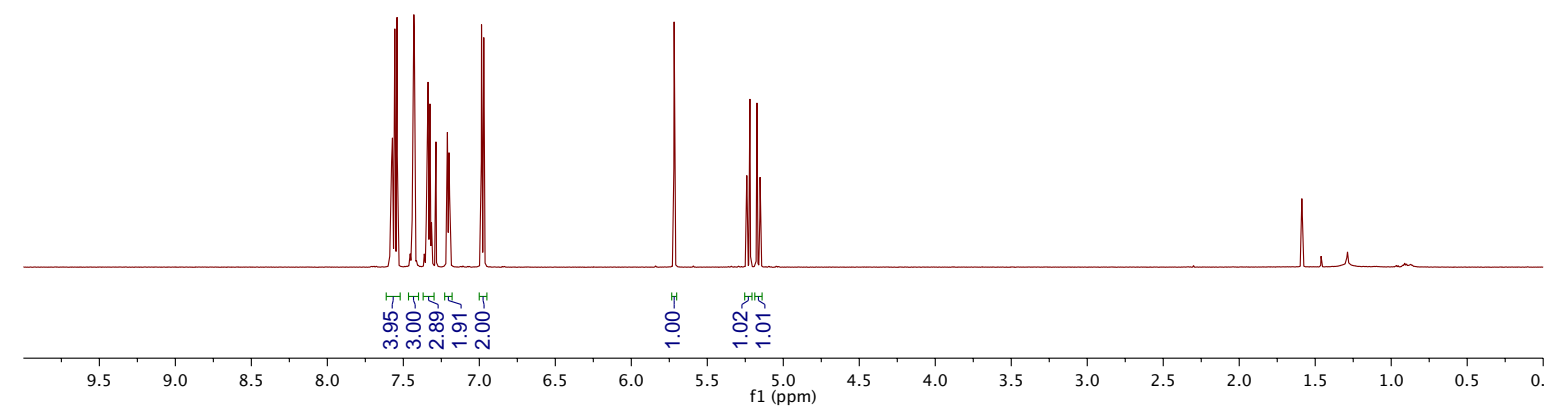

${ }^{13}$ C NMR (151 MHz, Chloroform- $d$ )

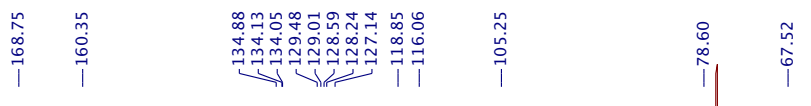<smiles>N#Cc1ccc(OC(C(=O)OCc2ccccc2)c2ccccc2)cc1</smiles>

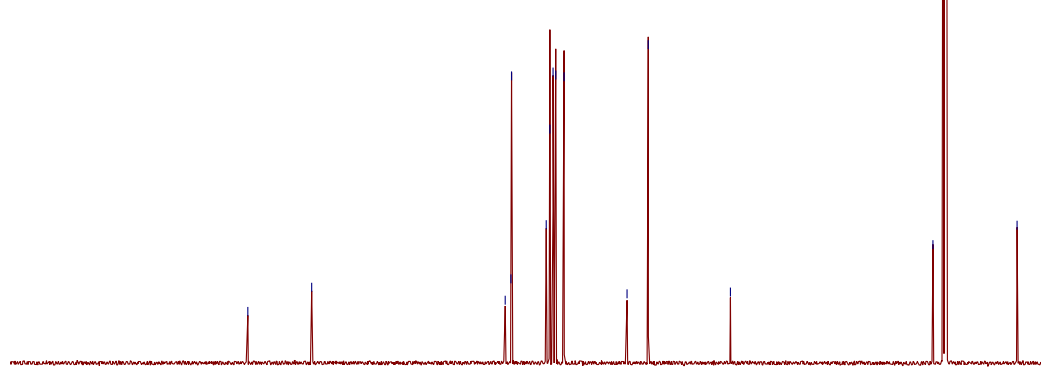

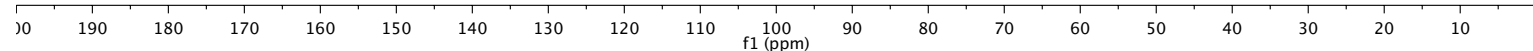


Cyclohexyl 2-(4-cyanophenoxy)-2-phenylacetate (12e)

${ }^{1}$ H NMR (600 MHz, Chloroform- $d$ )<smiles>N#Cc1ccc(OC(C(=O)OCc2ccccc2)c2ccccc2)cc1</smiles>

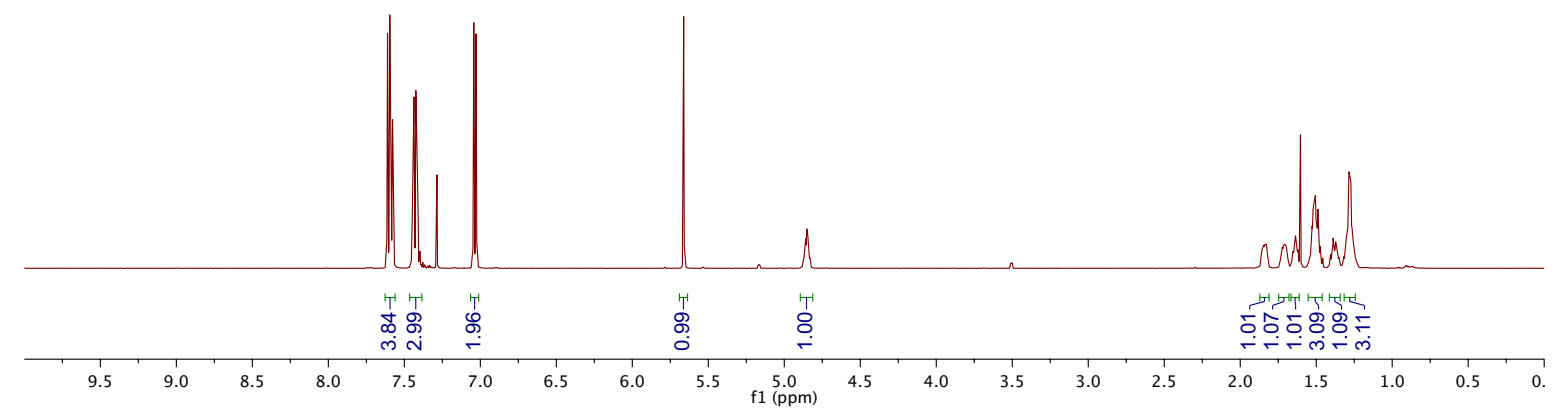

${ }^{13}$ C NMR (151 MHz, Chloroform- $d$ )

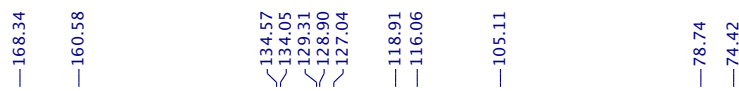

min<smiles>N#Cc1ccc(OC(C(=O)OCc2ccccc2)c2ccccc2)cc1</smiles>

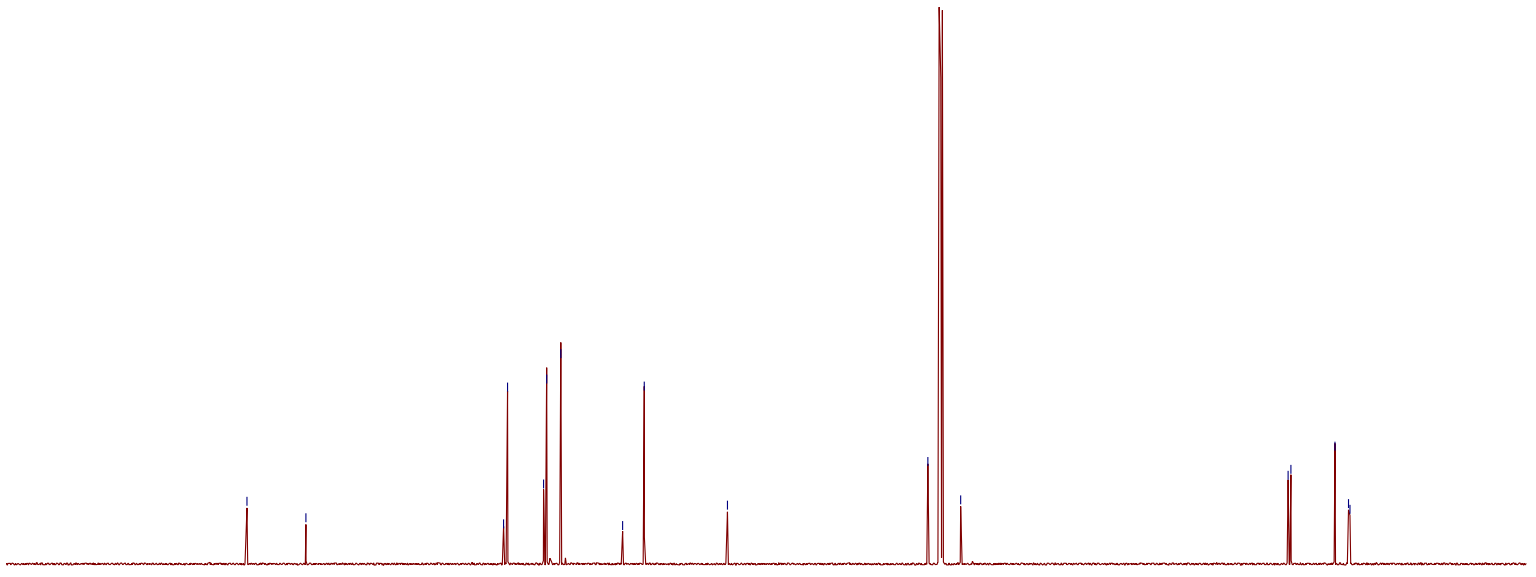

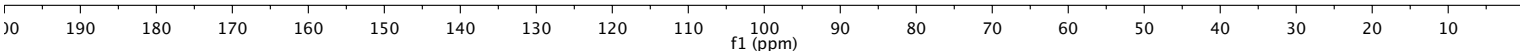


Methyl 2-(4-cyanophenoxy)-2-(4-fluorophenyl)acetate (12f)

${ }^{1}$ H NMR (600 MHz, Chloroform- $d$ )

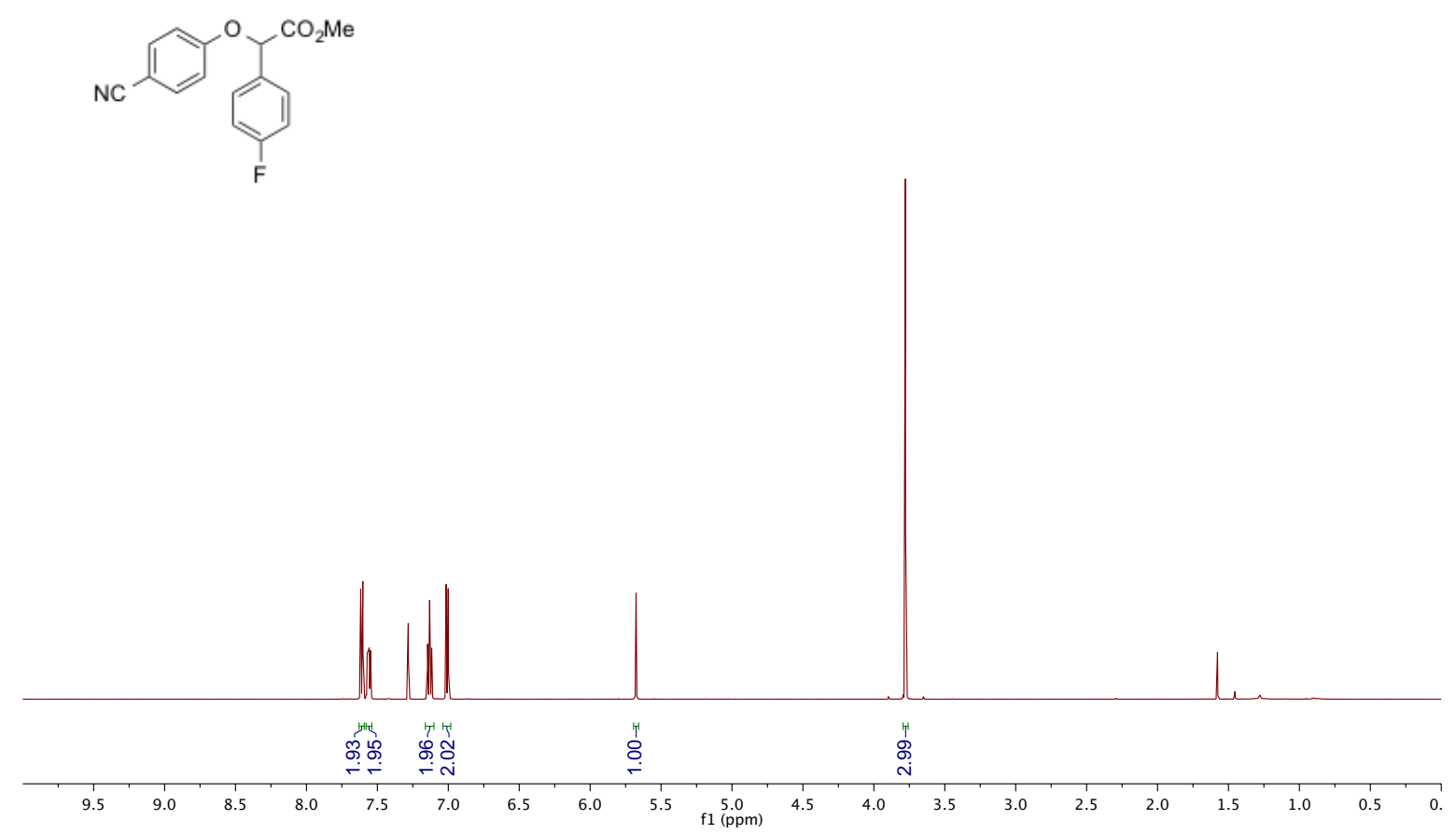

${ }^{13}$ C NMR (151 MHz, Chloroform- $\left.d\right)$

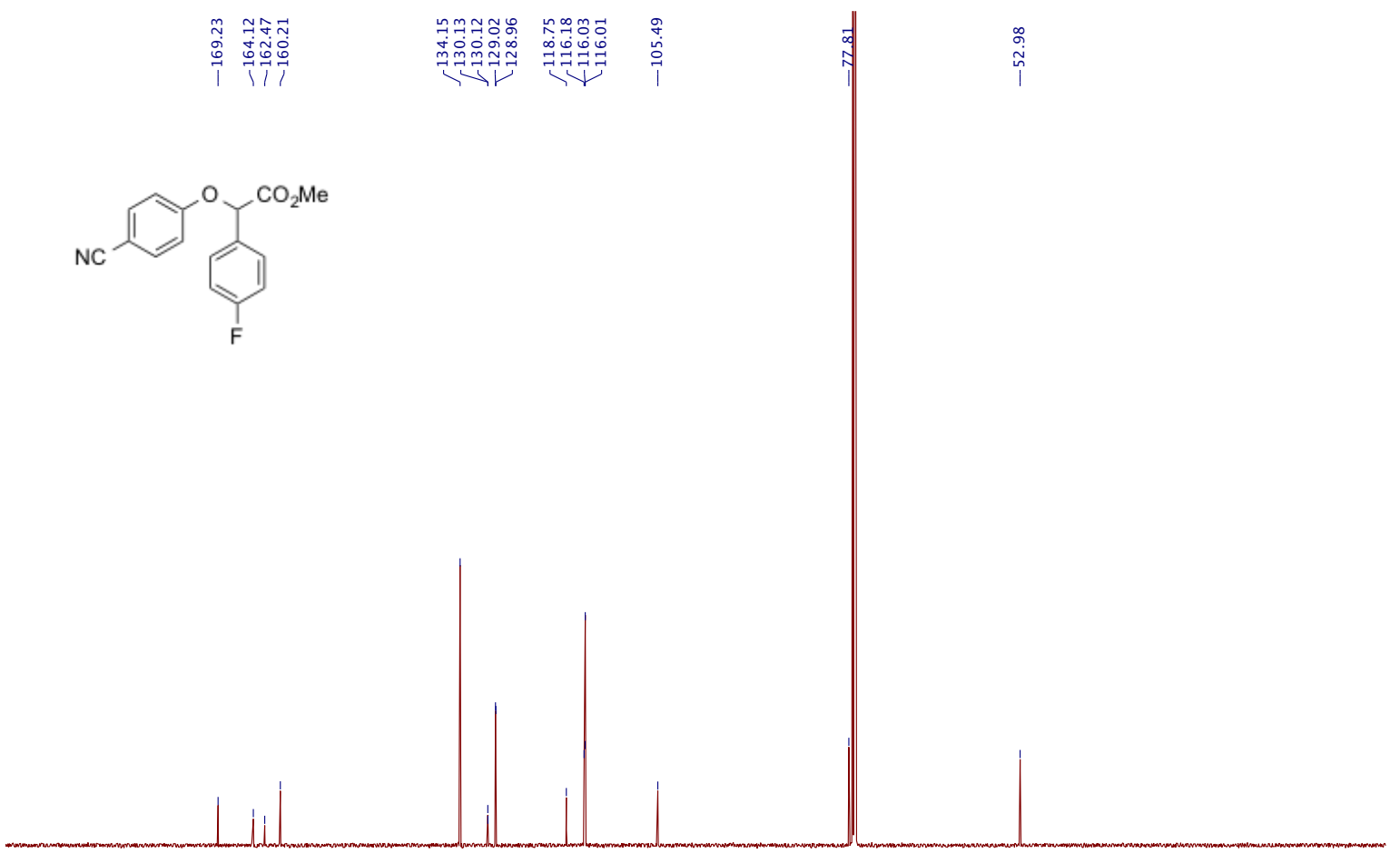

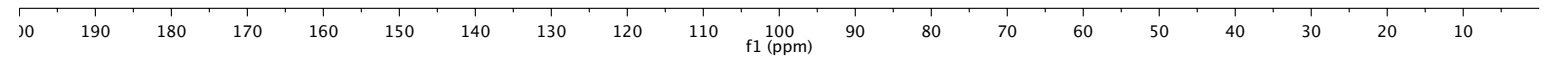


${ }^{19}$ F NMR (565 MHz, Chloroform- $d$ )

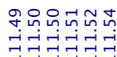

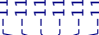

舟

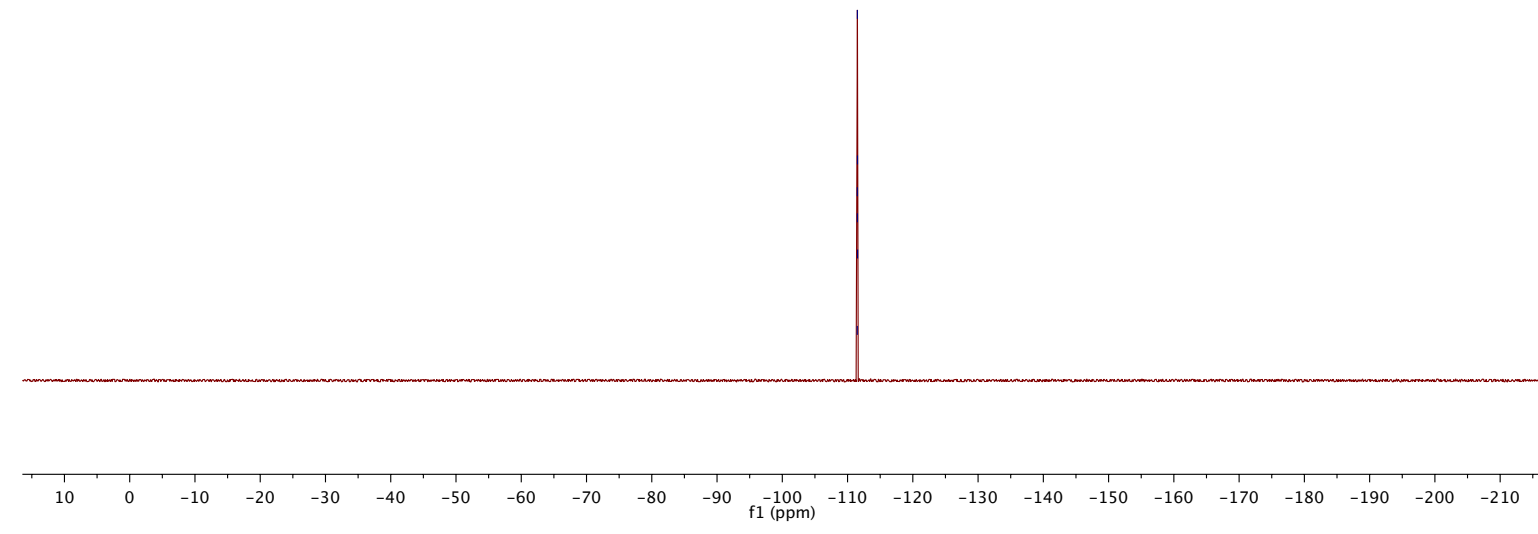


Methyl 2-(4-chlorophenyl)-2-(4-cyanophenoxy)acetate (12g)

${ }^{1}$ H NMR (600 MHz, Chloroform- $d$ )<smiles>CC(=O)Oc1ccc(Cl)cc1</smiles>

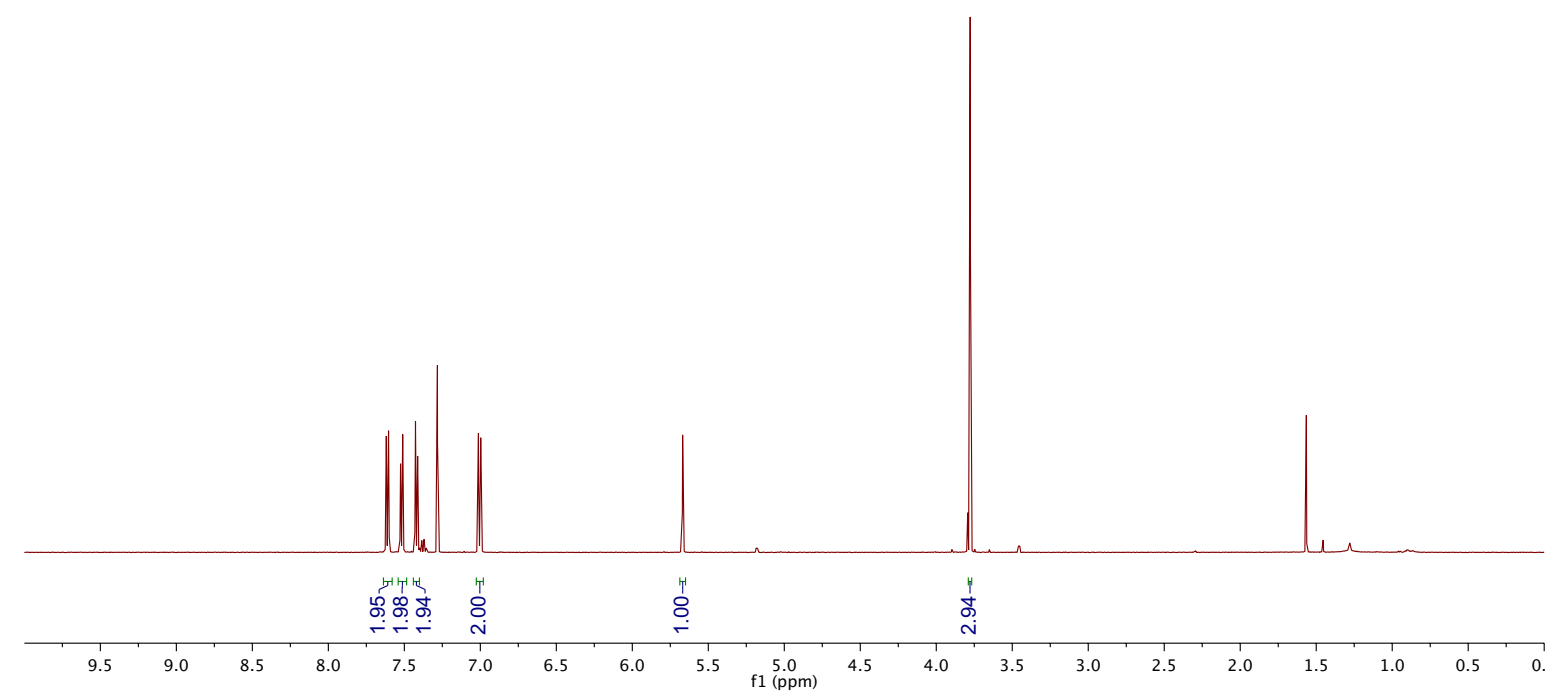

${ }^{13}$ C NMR (151 MHz, Chloroform- $d$ )
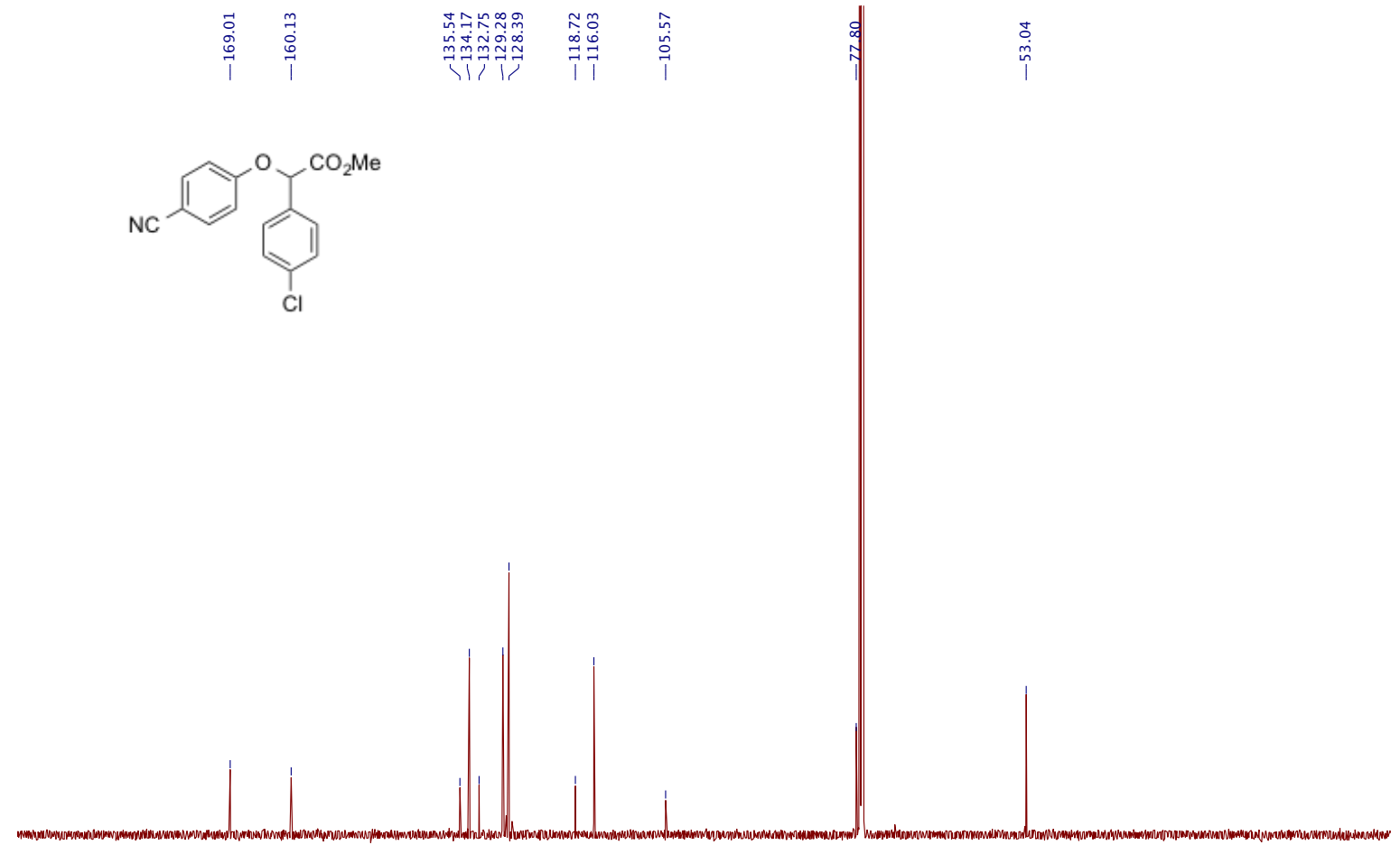

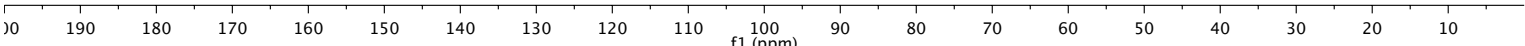


Methyl 2-(4-bromophenyl)-2-(4-cyanophenoxy)acetate (12h)

${ }^{1}$ H NMR (600 MHz, Chloroform- $d$ )<smiles>CC(=O)OC(Oc1ccc([N+]#N)cc1)c1ccc(Br)cc1</smiles>

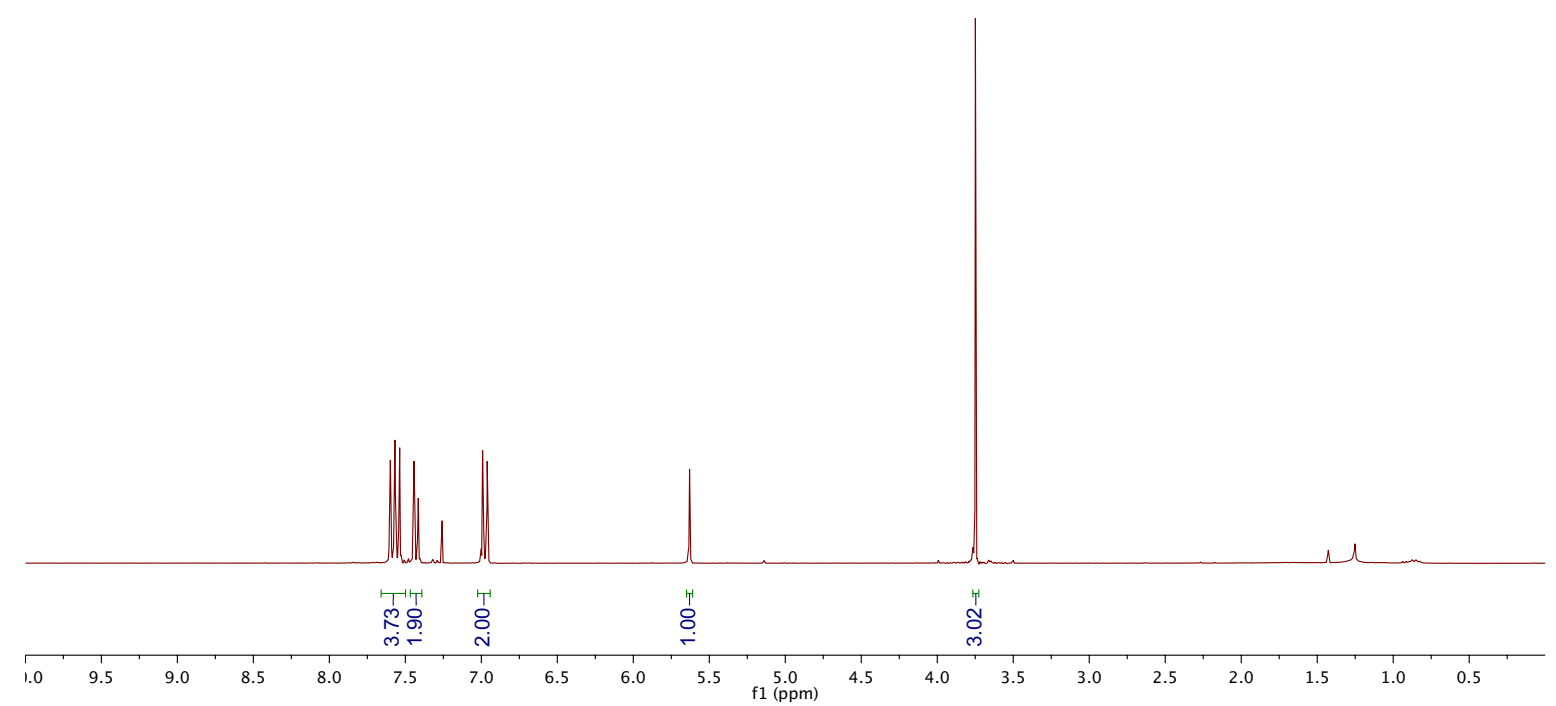

${ }^{13}$ C NMR (151 MHz, Chloroform- $d$ )
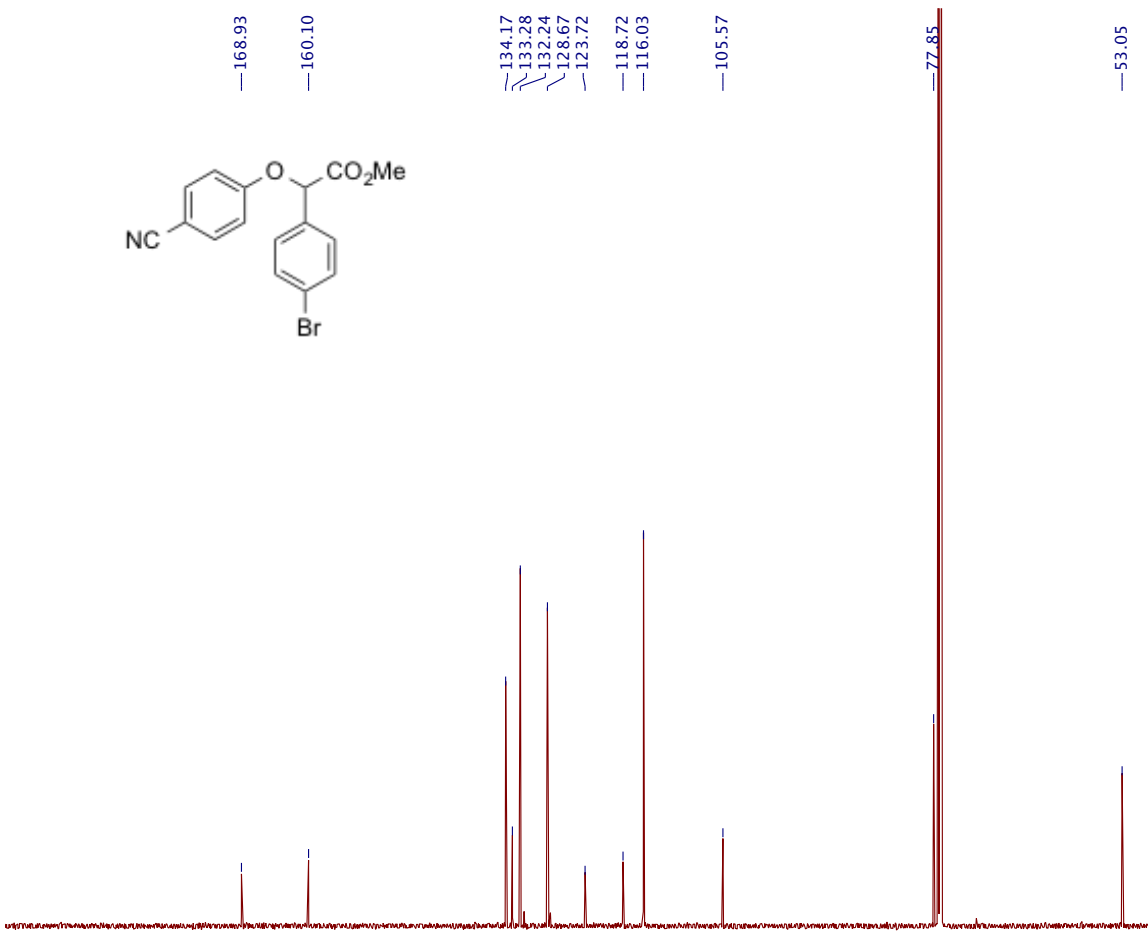

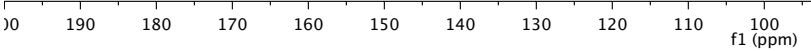


Methyl 2-(4-cyanophenoxy)-2-(4-methoxyphenyl)acetate (12i)

${ }^{1}$ H NMR (600 MHz, Chloroform- $\left.d\right)$
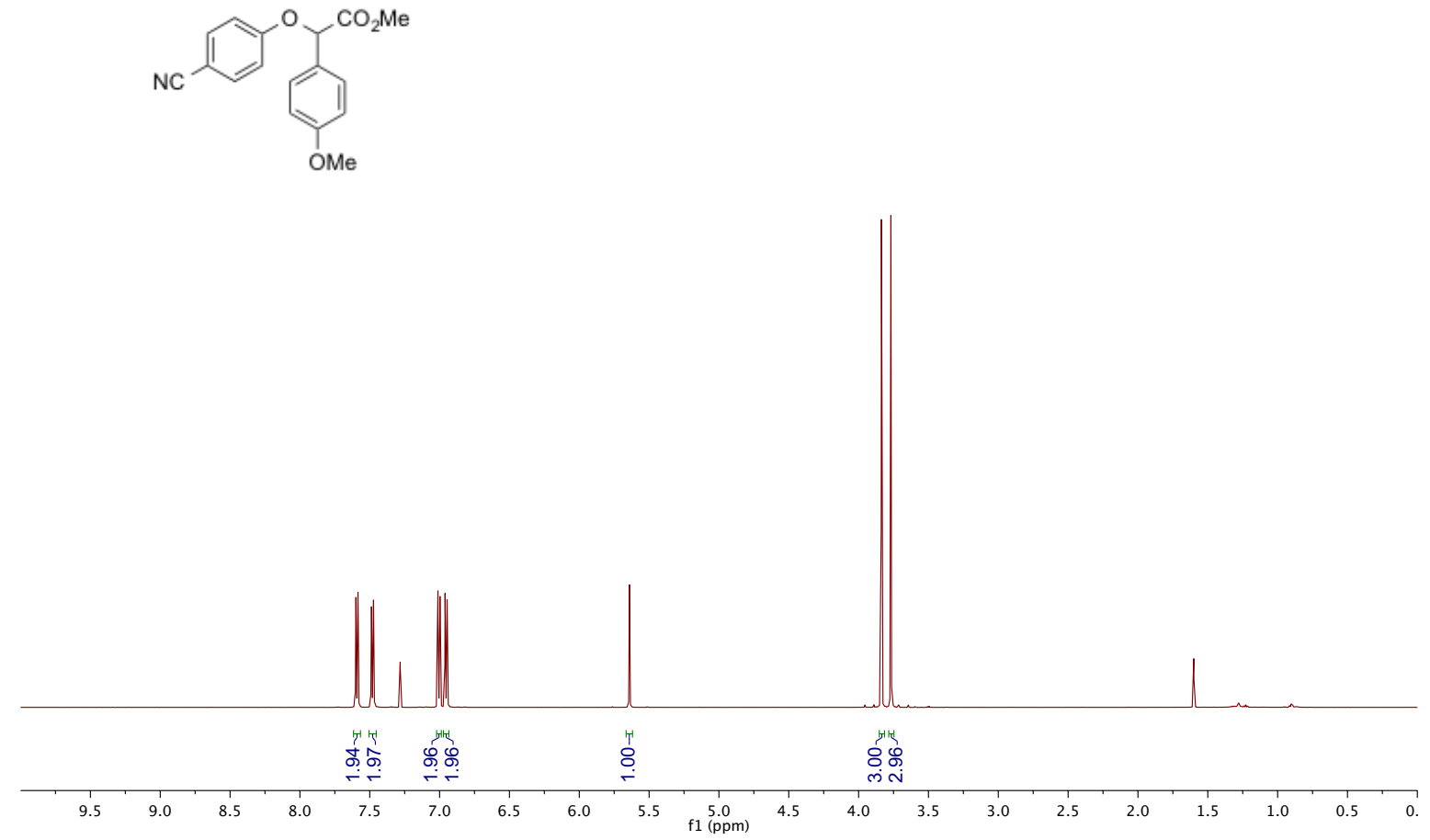

${ }^{13}$ C NMR (151 MHz, Chloroform- $d$ )

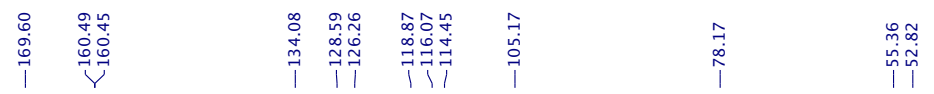
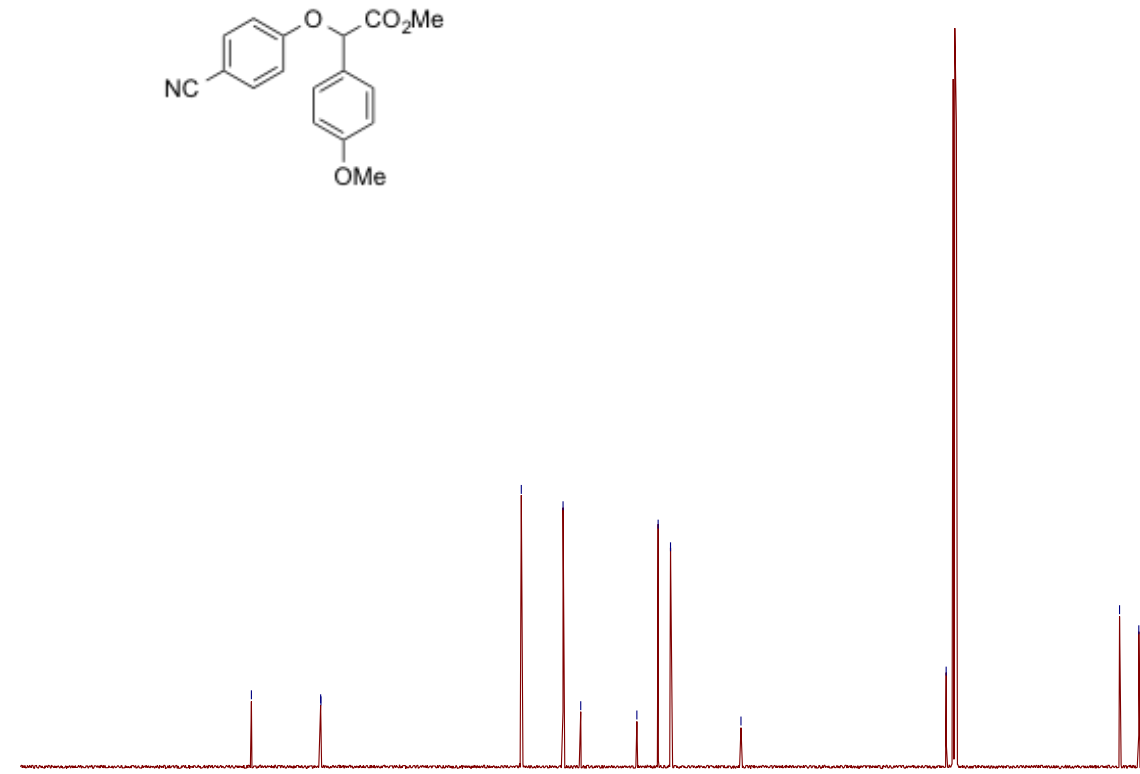

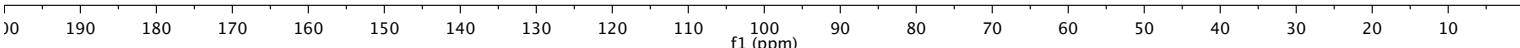


Methyl 2-(3-chlorophenyl)-2-(4-cyanophenoxy)acetate (12j)

${ }^{1}$ H NMR (600 MHz, Chloroform- $d$ )

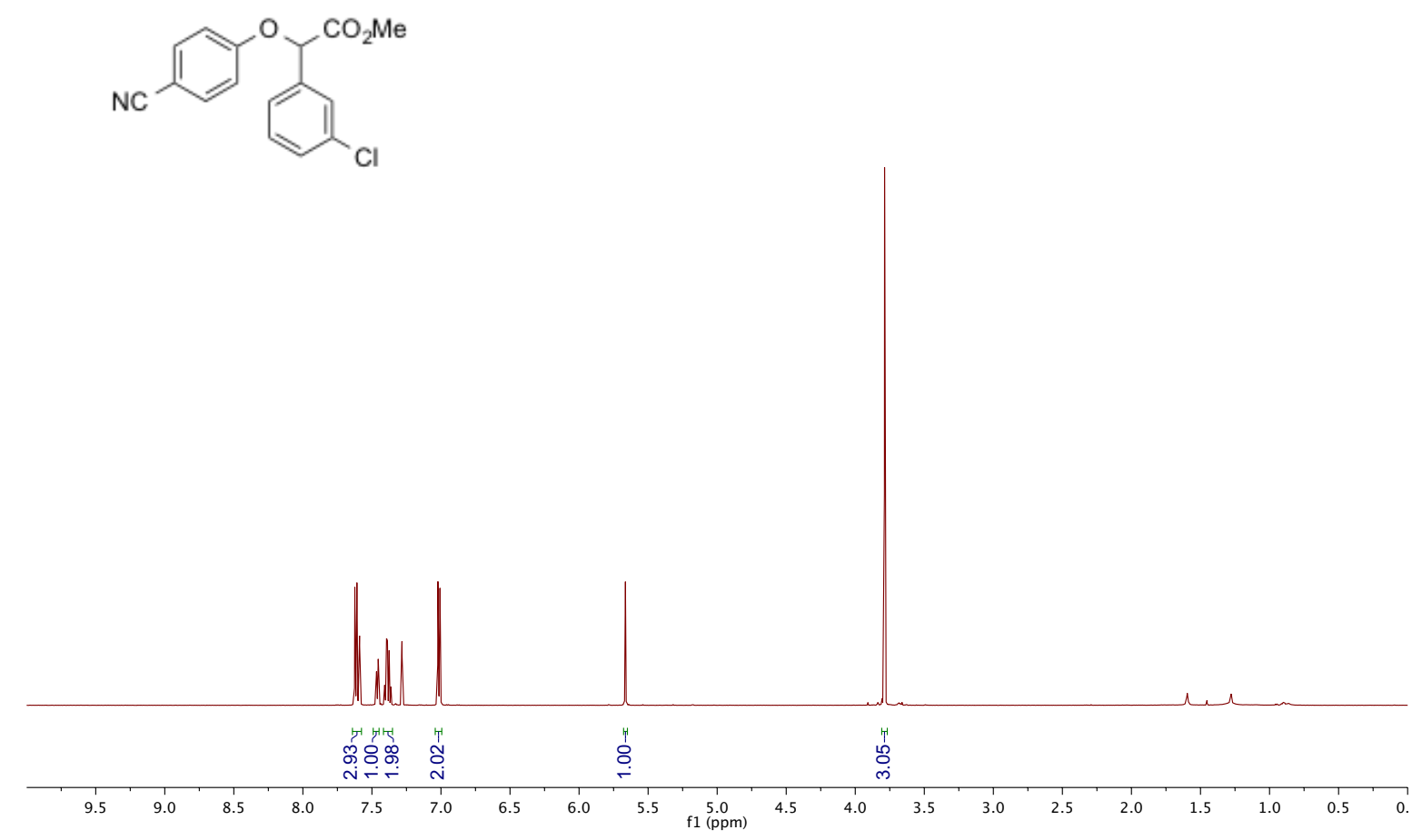

${ }^{13}$ C NMR (151 MHz, Chloroform- $d$ )
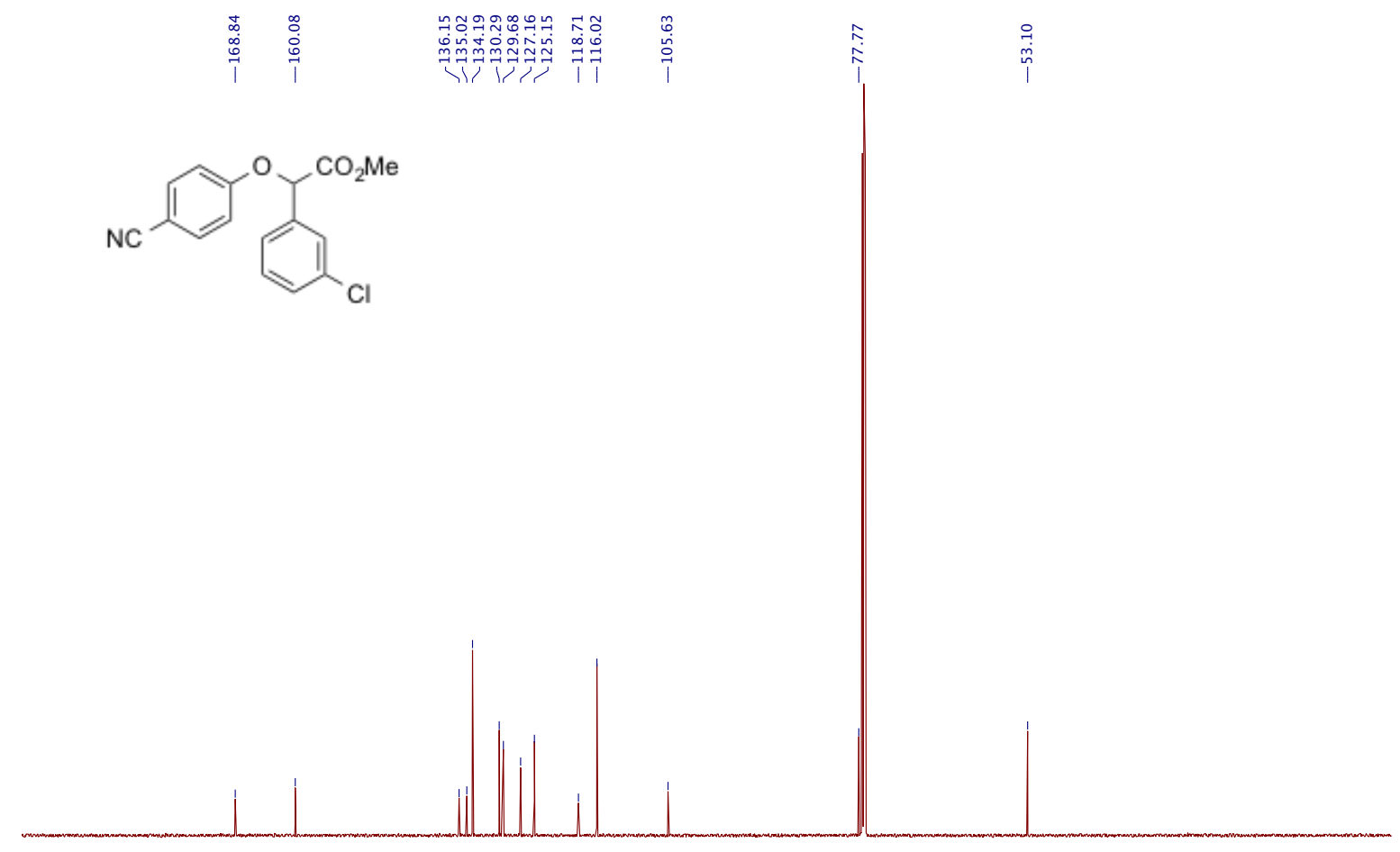

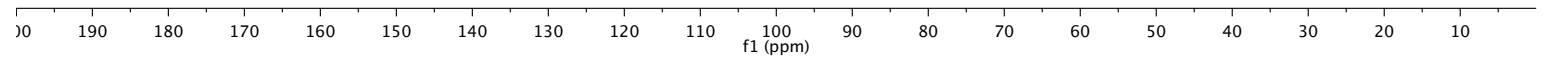


Methyl 2-(4-cyanophenoxy)-2-(3-methoxyphenyl)acetate (12k)

${ }^{1}$ H NMR (600 MHz, Chloroform- $\left.d\right)$
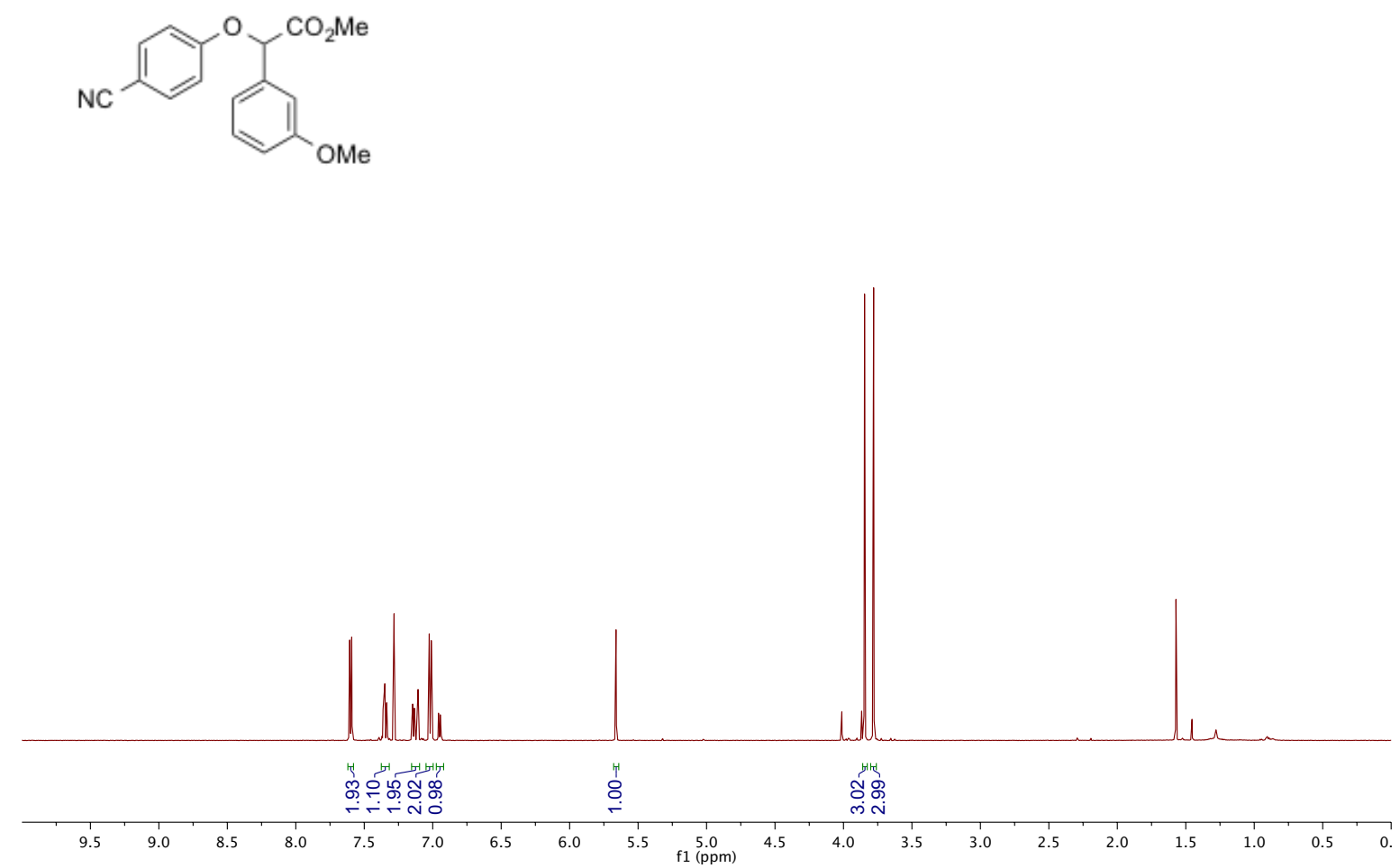

${ }^{13}$ C NMR (151 MHz, Chloroform- $d$ )
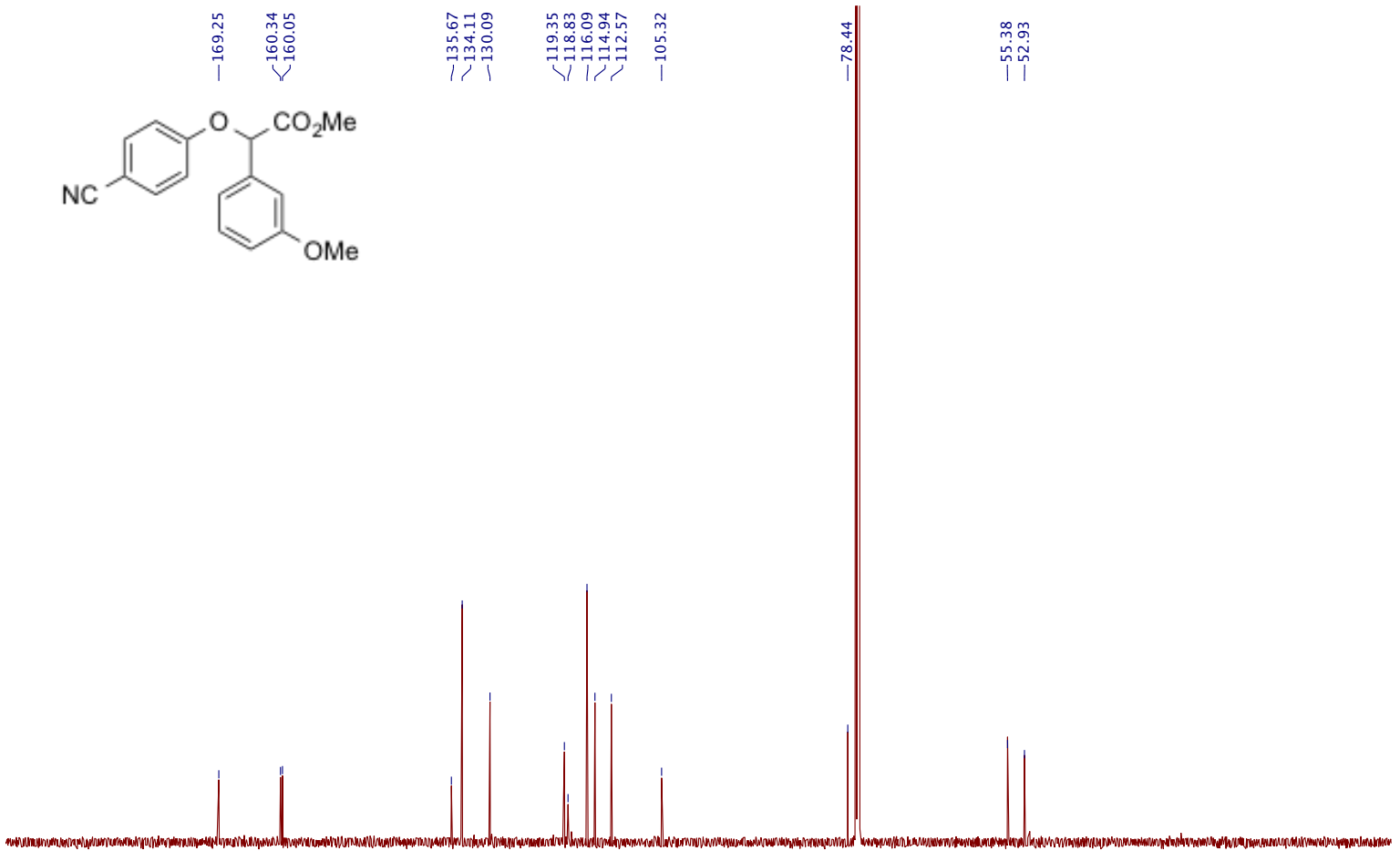

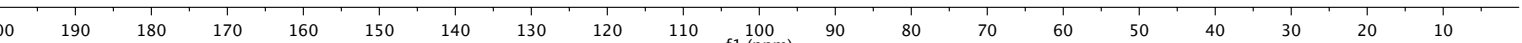


Methyl 2-(4-cyanophenoxy)-2-(2-fluorophenyl)acetate (12I)

${ }^{1}$ H NMR (600 MHz, Chloroform-d)<smiles>CC(=O)C(Oc1ccc(C#N)cc1)c1ccccc1F</smiles>

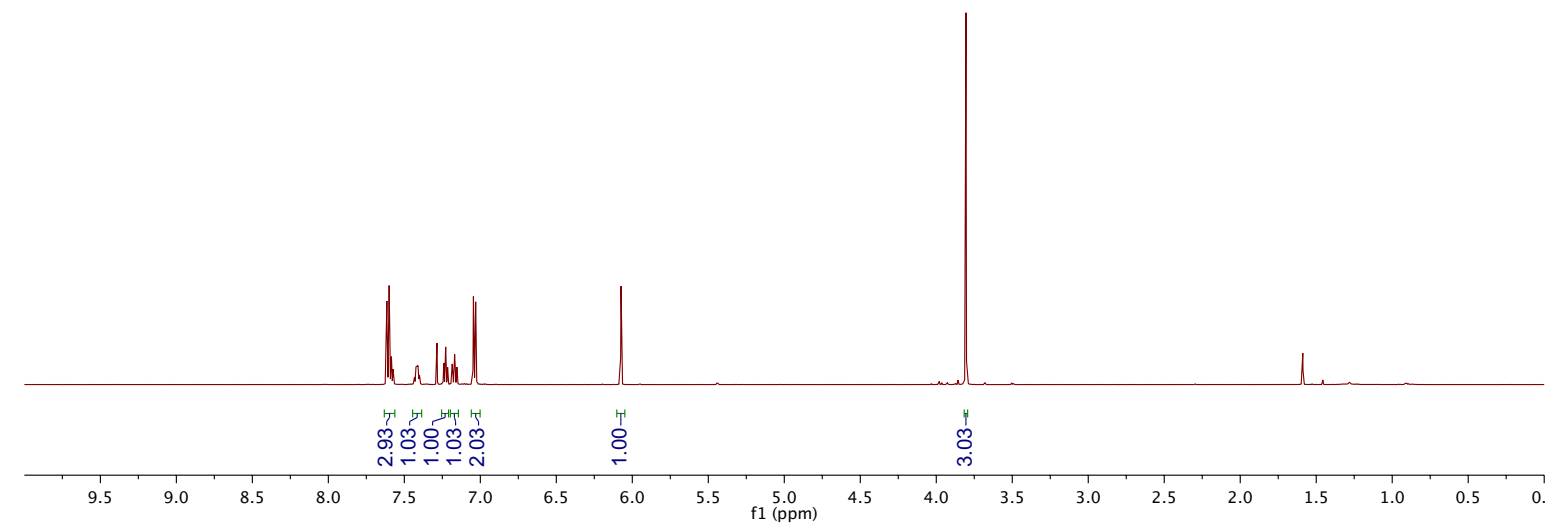

${ }^{13}$ C NMR (151 MHz, Chloroform- $d$ )<smiles>CC(=O)C(Oc1ccc(C#N)cc1)c1ccccc1F</smiles>

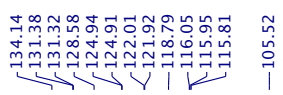
管

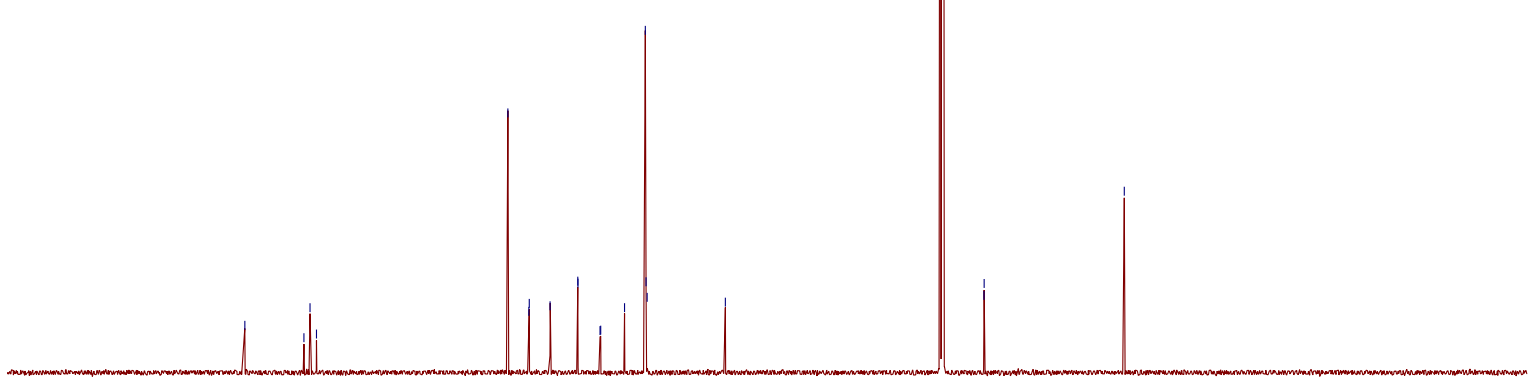

10 
${ }^{19}$ F NMR (565 MHz, Chloroform- $d$ )

nots

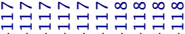

NC

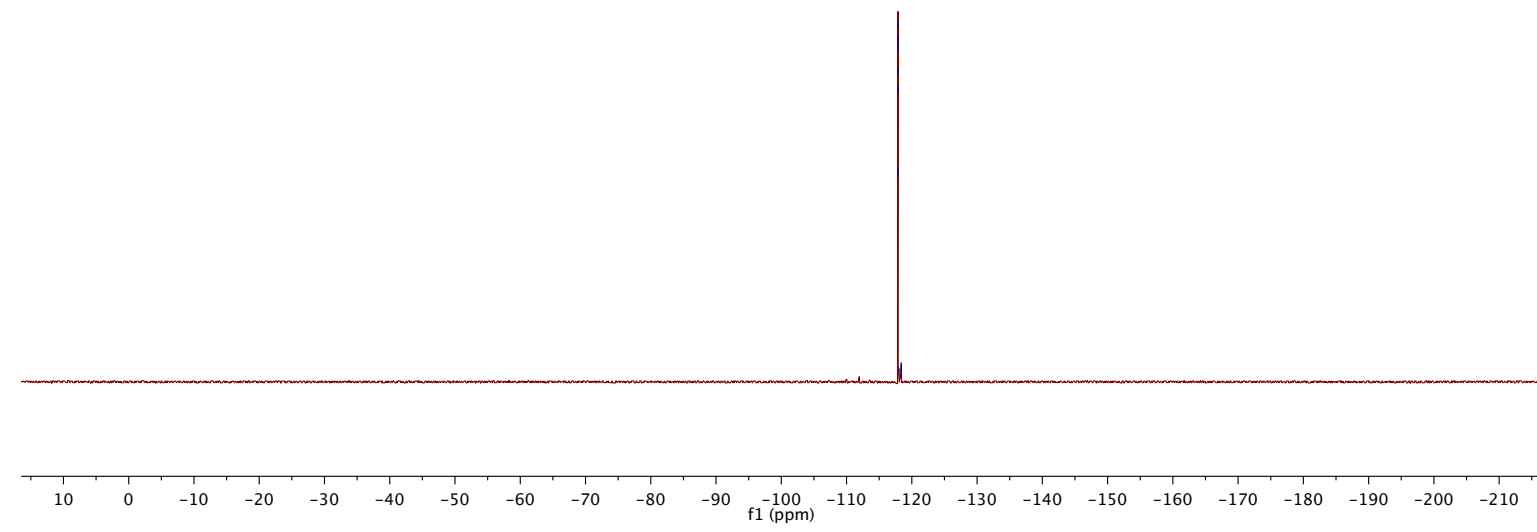


Methyl 2-(2-chlorophenyl)-2-(4-cyanophenoxy)acetate (12m)

${ }^{1}$ H NMR (600 MHz, Chloroform- $d$ )<smiles>CC(=O)OC(Oc1ccc(C#N)cc1)c1ccccc1Cl</smiles>

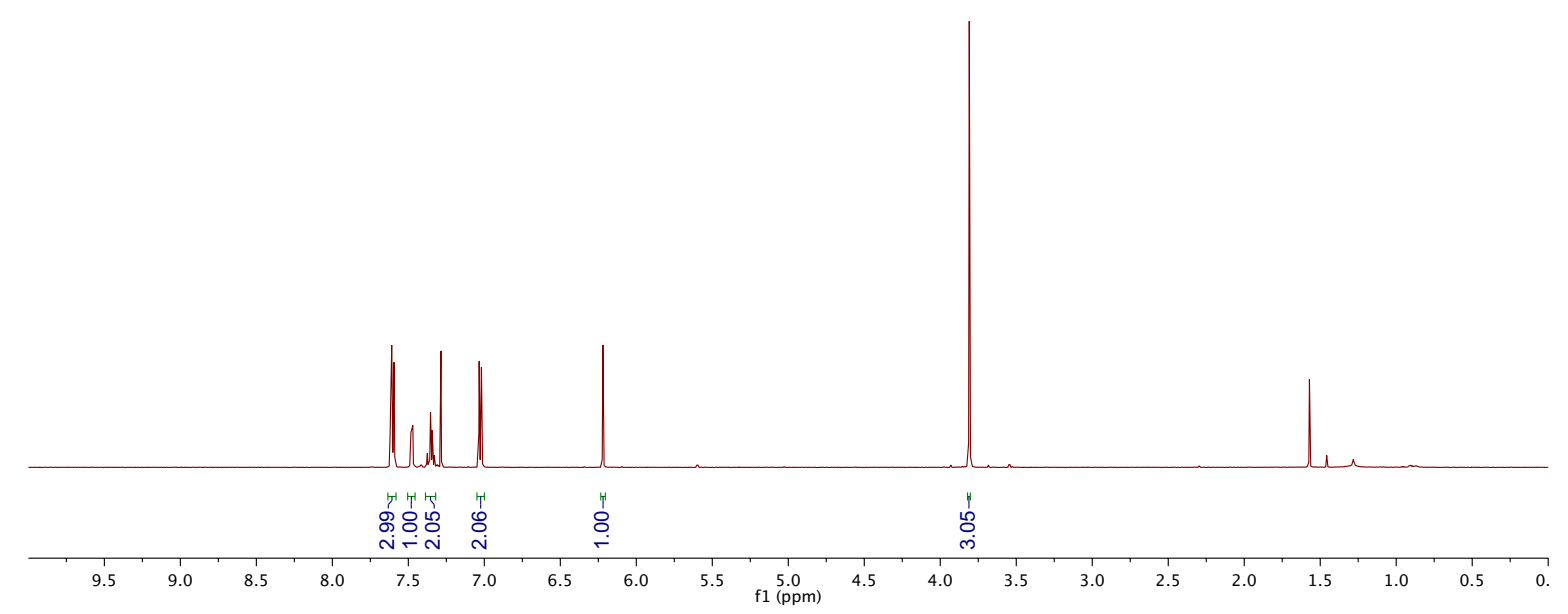

${ }^{13}$ C NMR (151 MHz, Chloroform- $d$ )

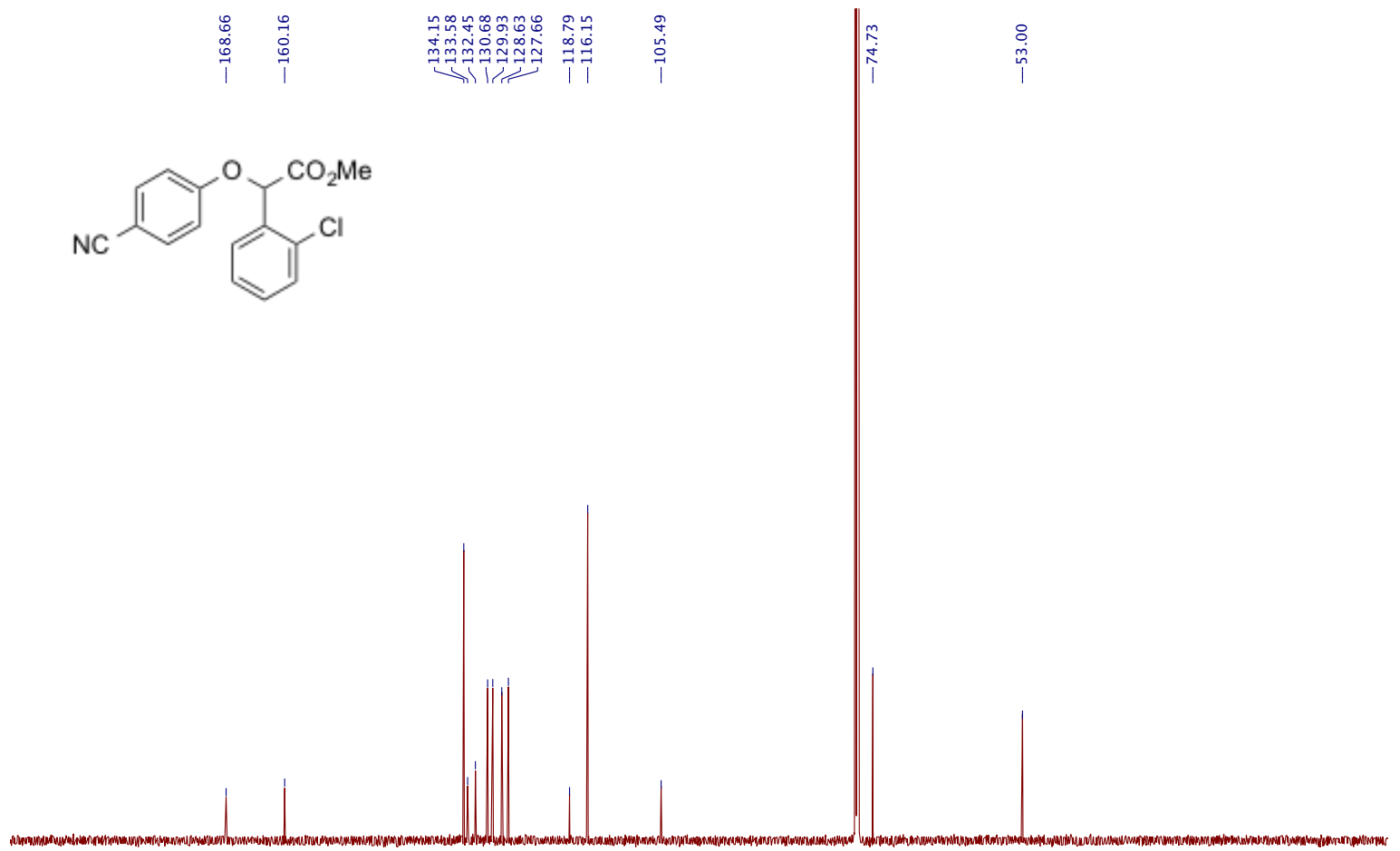

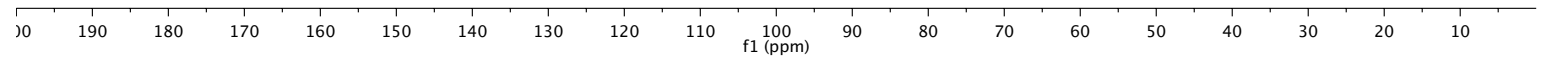


Methyl 2-(4-cyanophenoxy)-2-(naphthalen-2-yl)acetate (12n)

${ }^{1}$ H NMR (600 MHz, Chloroform-d)
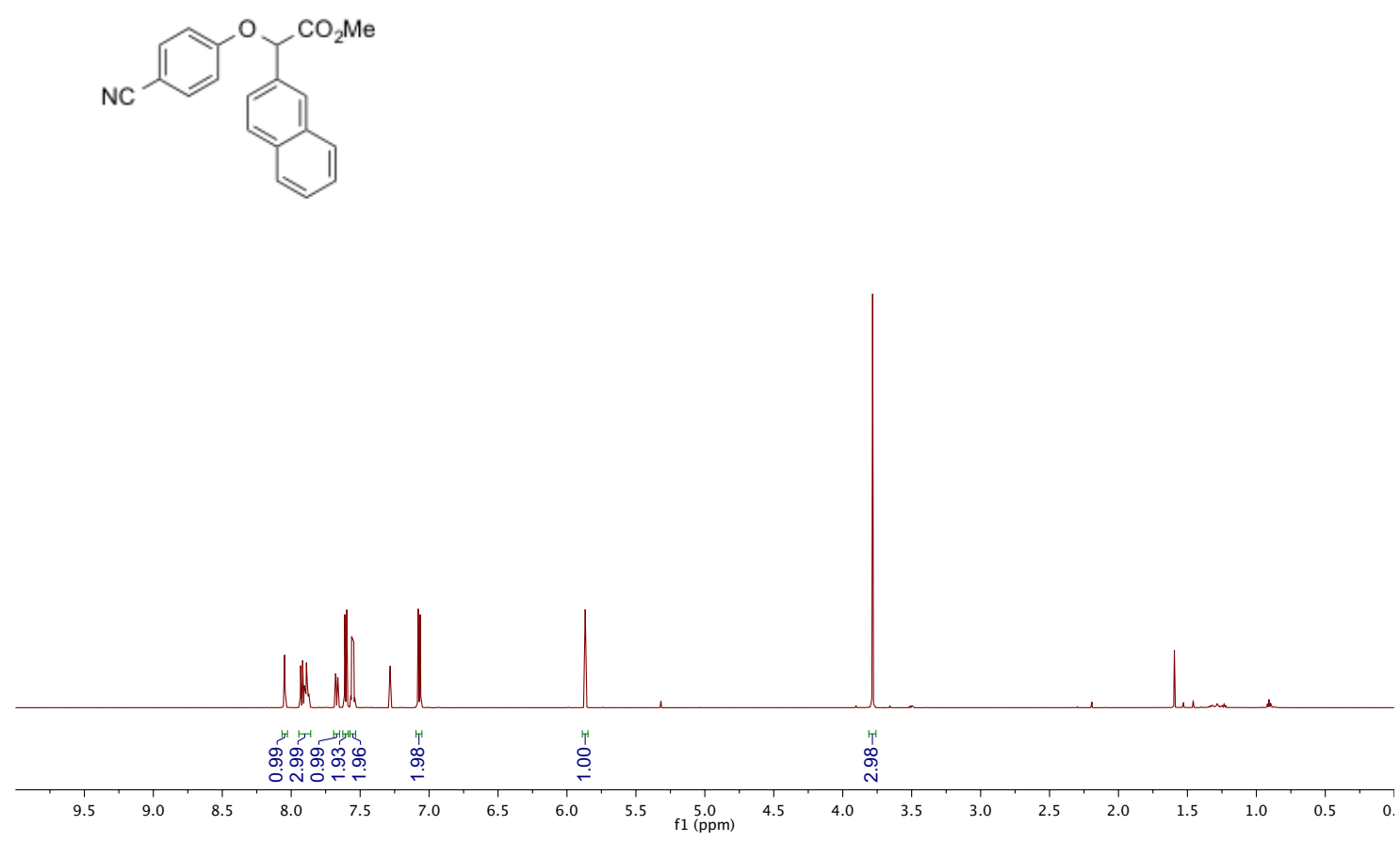

${ }^{13}$ C NMR (151 MHz, Chloroform- $d$ )

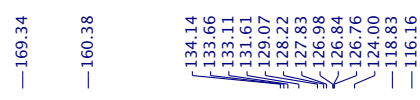

$\stackrel{\substack{n \\ n}}{n}$

$\underset{\substack{i \\ \infty}}{\substack{\infty \\ \hdashline}}$

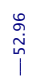
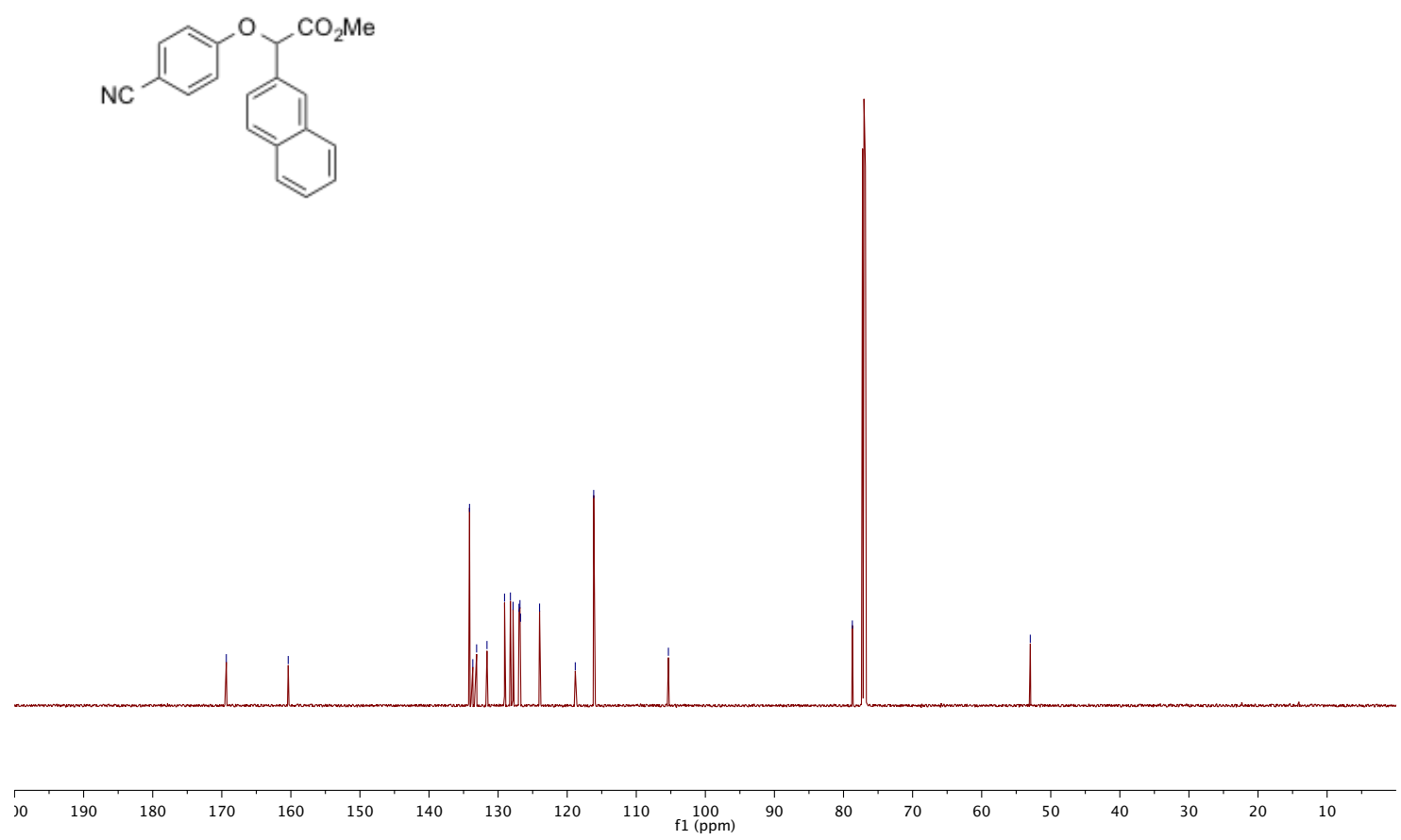

S67 
Methyl 2-(4-cyanophenoxy)-2-(naphthalen-1-yl)acetate (120)

${ }^{1}$ H NMR (600 MHz, Chloroform- $d$ )
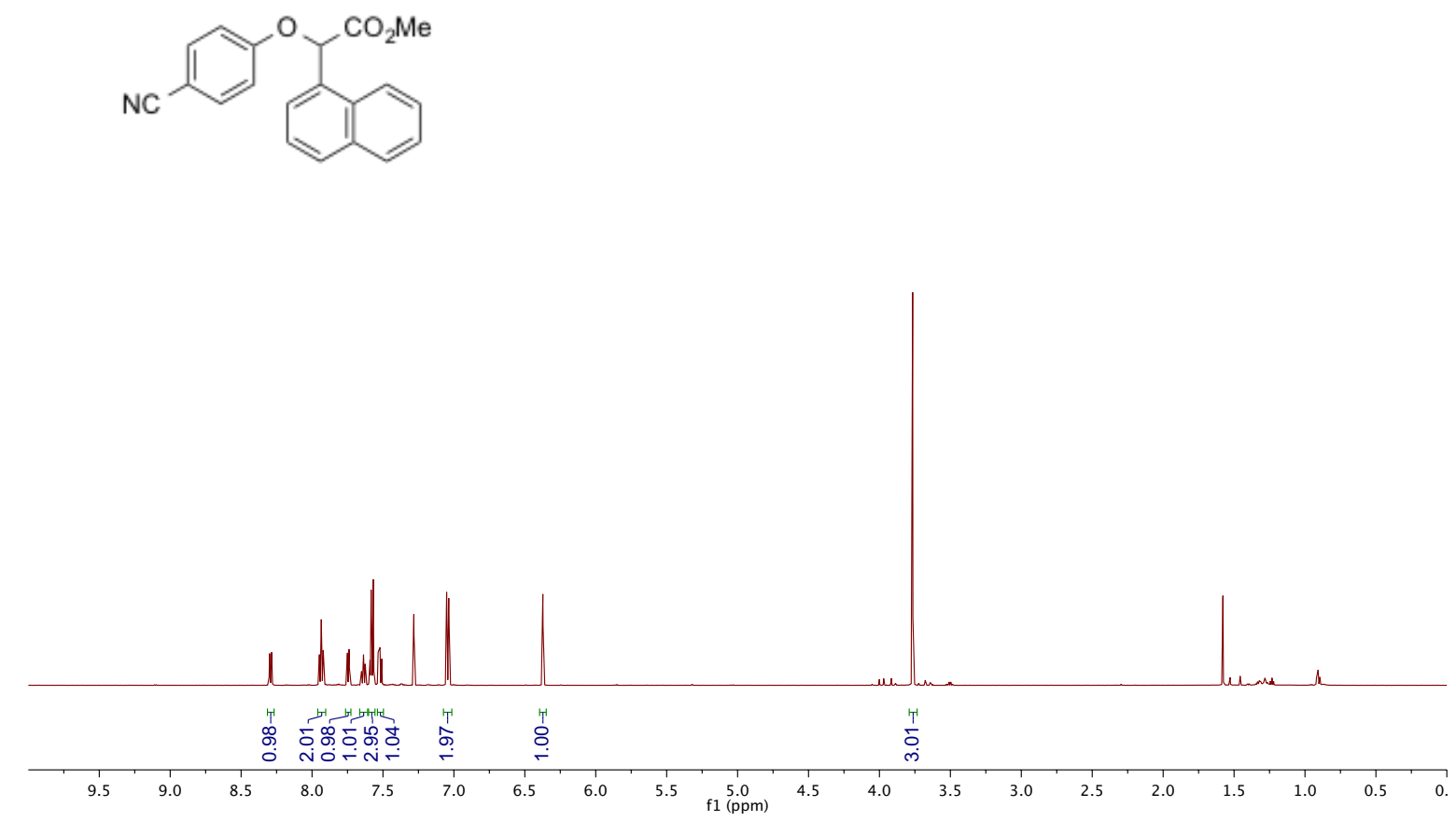

${ }^{13}$ C NMR (151 MHz, Chloroform- $d$ )
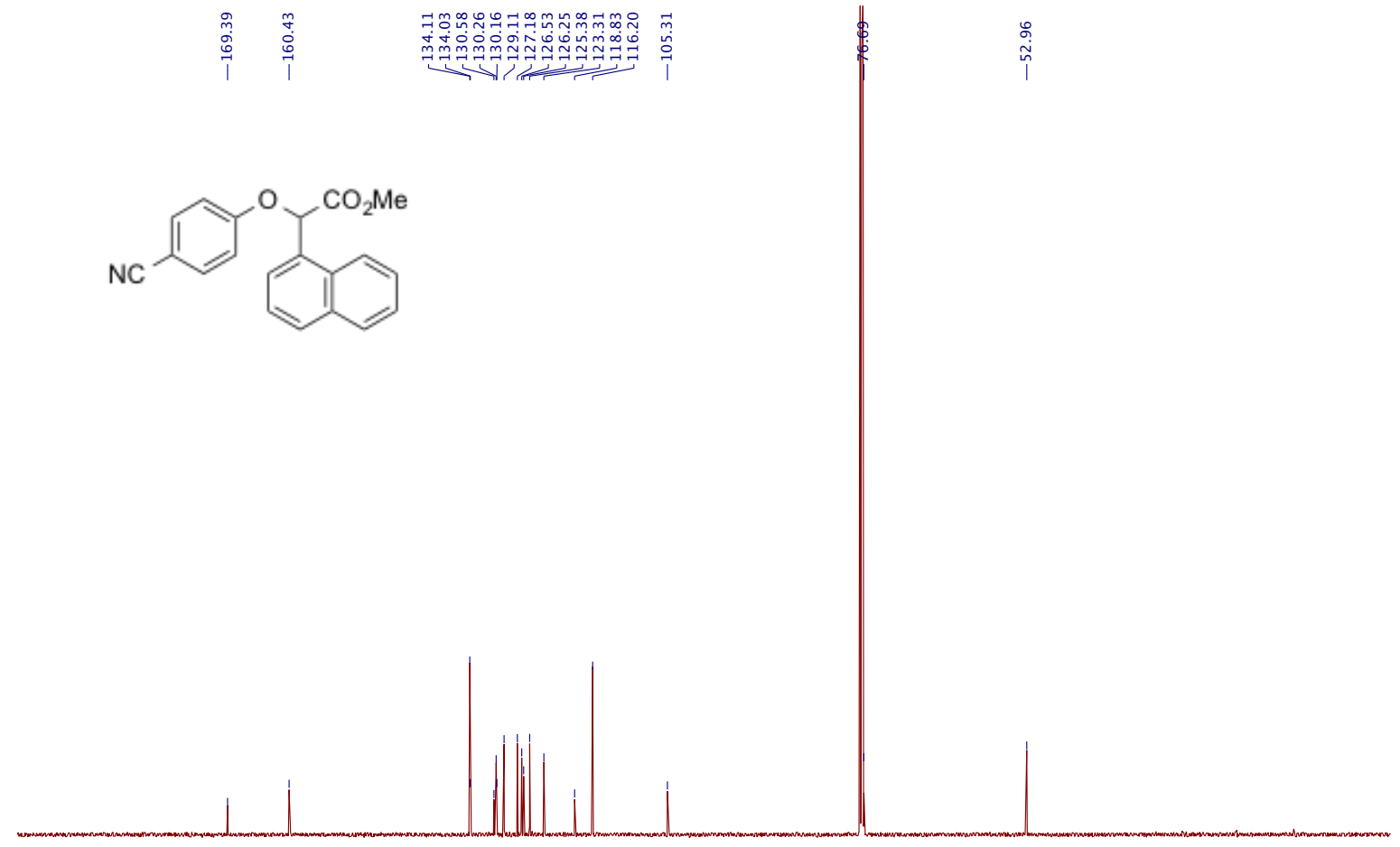

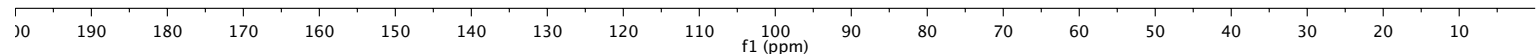


Methyl 2-(benzo[d][1,3]dioxol-5-yl)-2-(4-cyanophenoxy)acetate (12p)

${ }^{1}$ H NMR (600 MHz, Chloroform- $\left.d\right)$

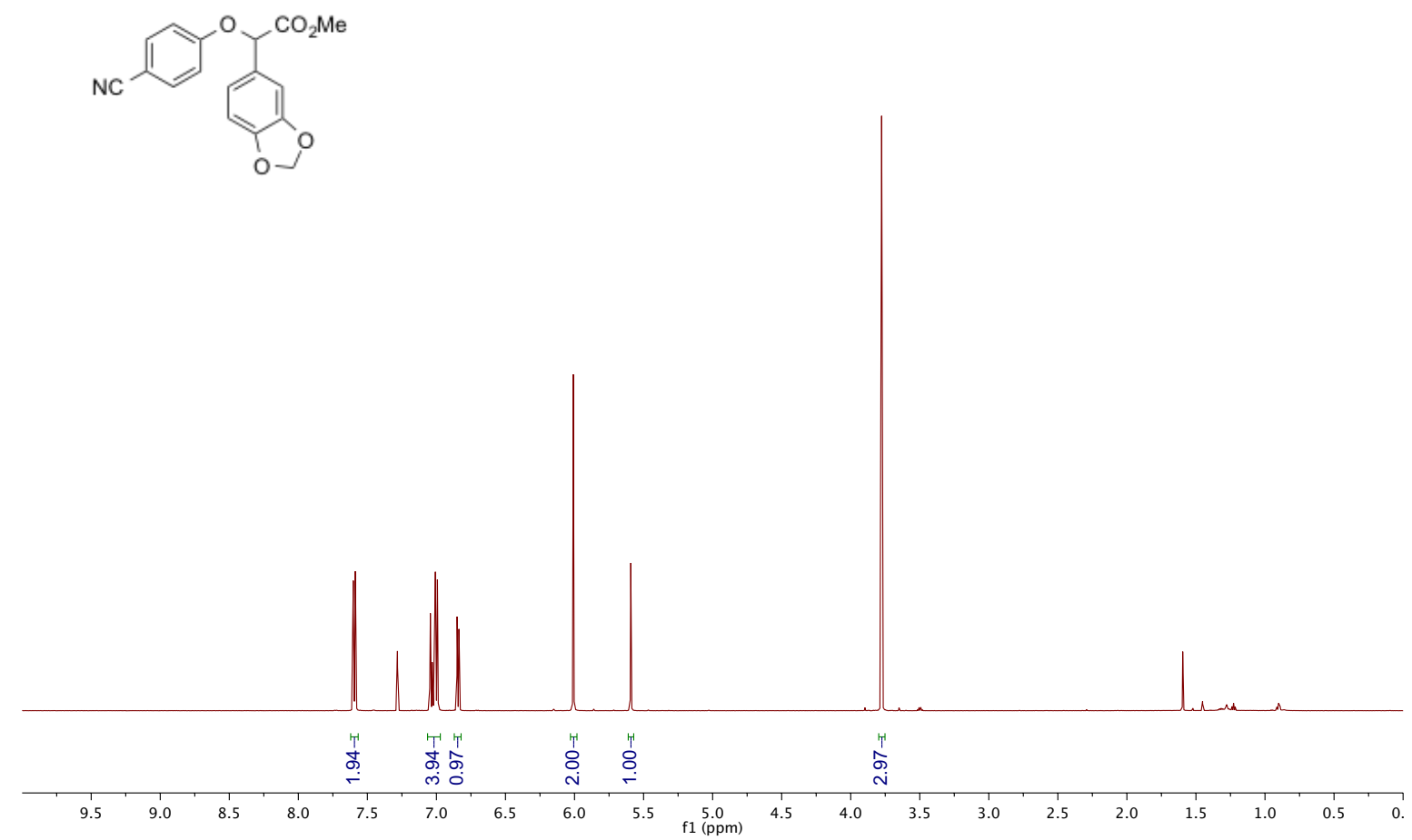

${ }^{13}$ C NMR (151 MHz, Chloroform- $d$ )

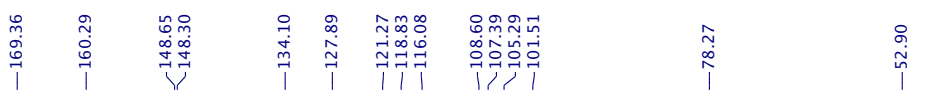
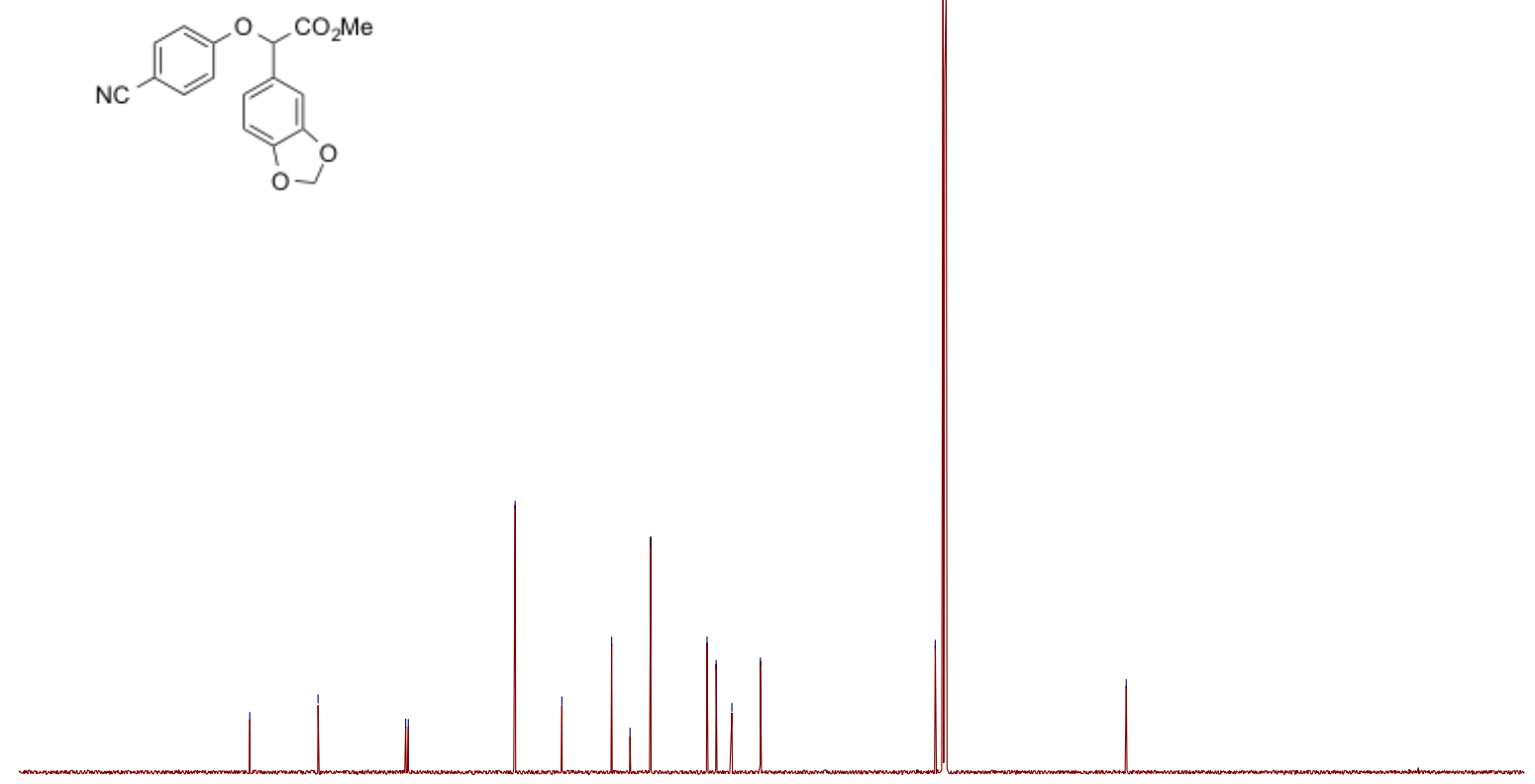

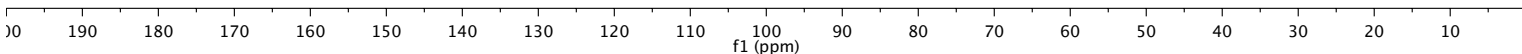


Methyl 2-(4-cyanophenoxy)-2-(pyridin-3-yl)acetate (12q)

${ }^{1}$ H NMR (400 MHz, Chloroform- $d$ )

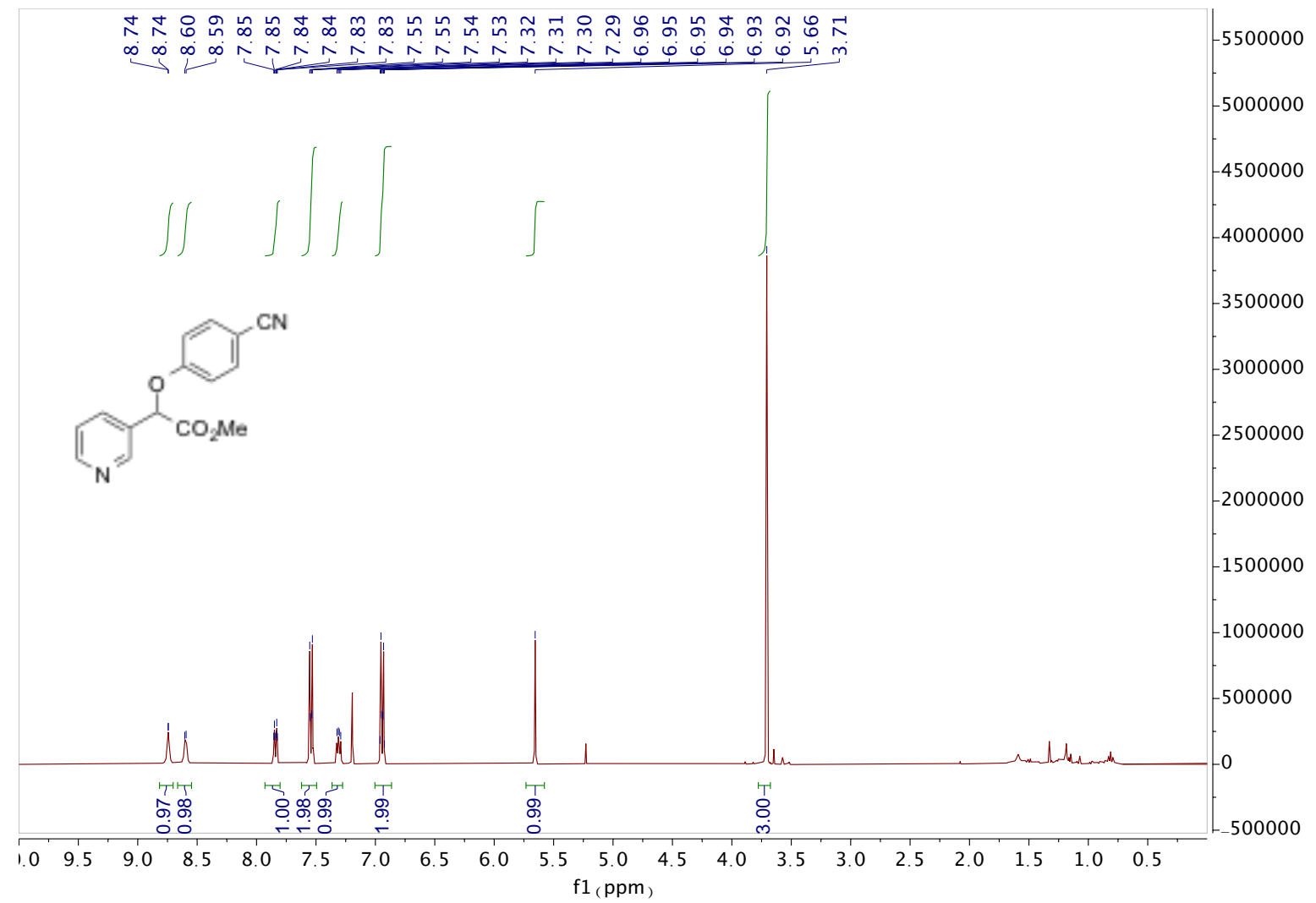

${ }^{13}$ C NMR (101 MHz, Chloroform- $d$ )

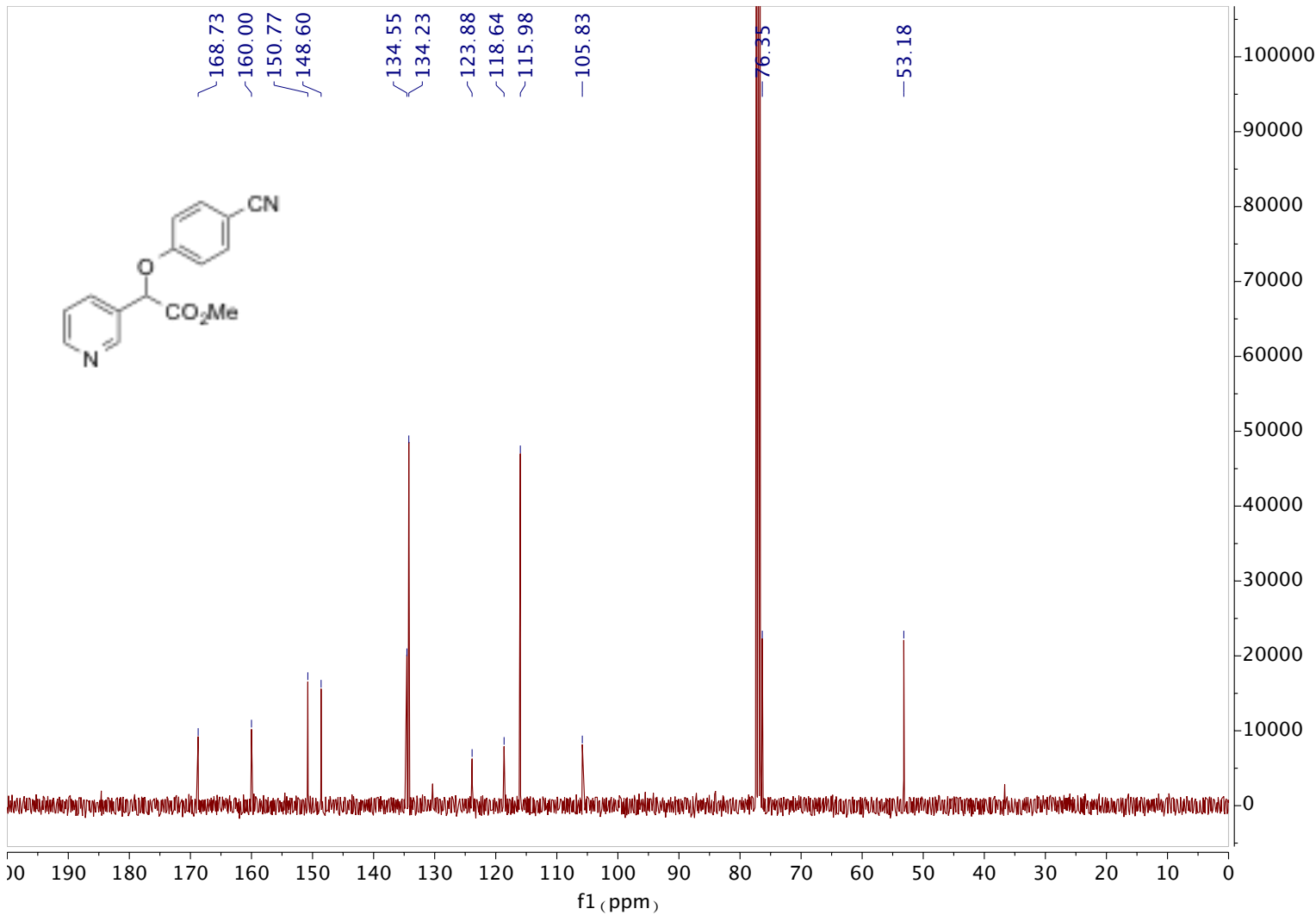


Methyl 2-phenoxy-2-(thiophen-2-yl)acetate (12r)

${ }^{1}$ H NMR (400 MHz, Chloroform- $d$ )

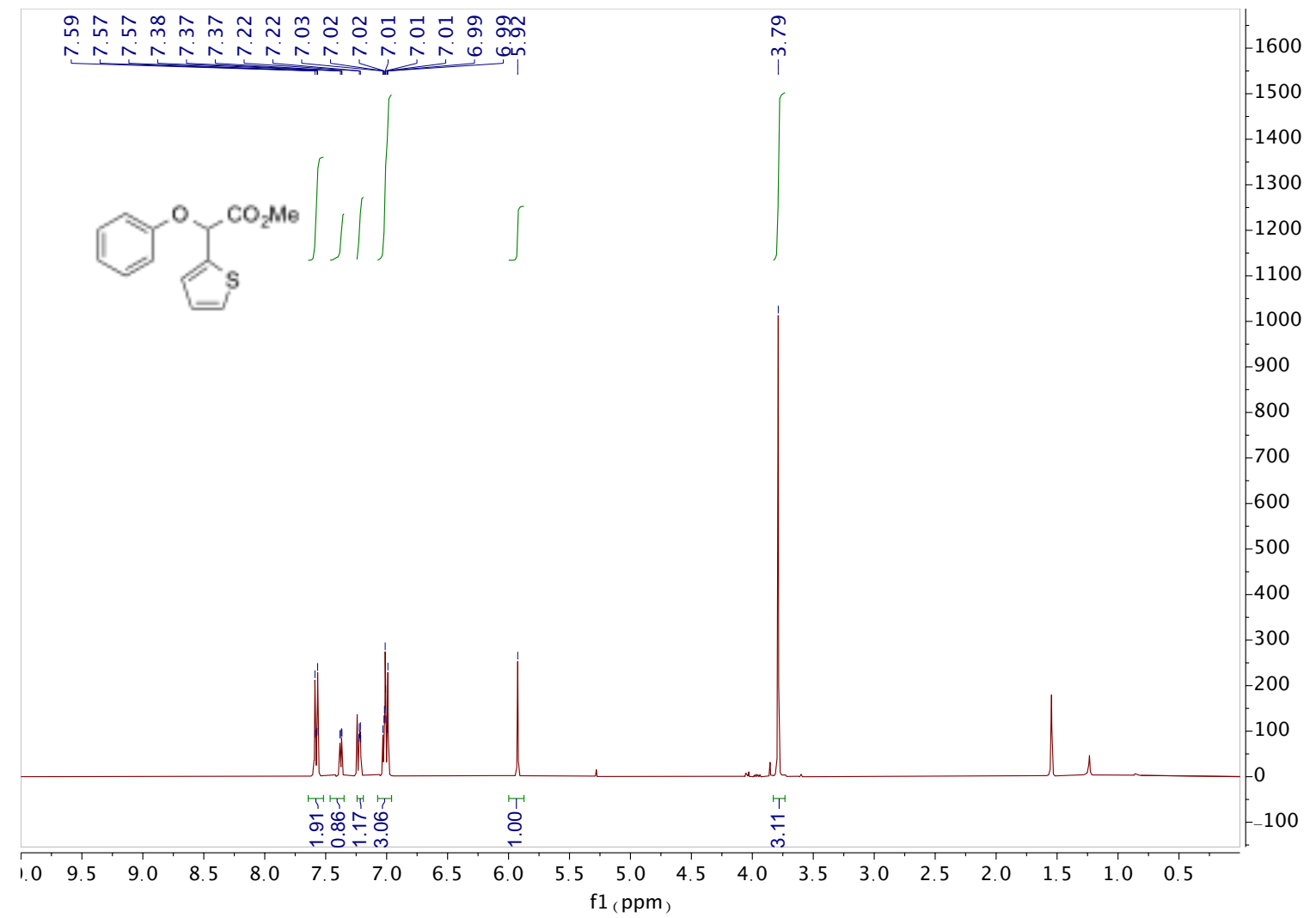

${ }^{13}$ C NMR (101 MHz, Chloroform- $d$ )

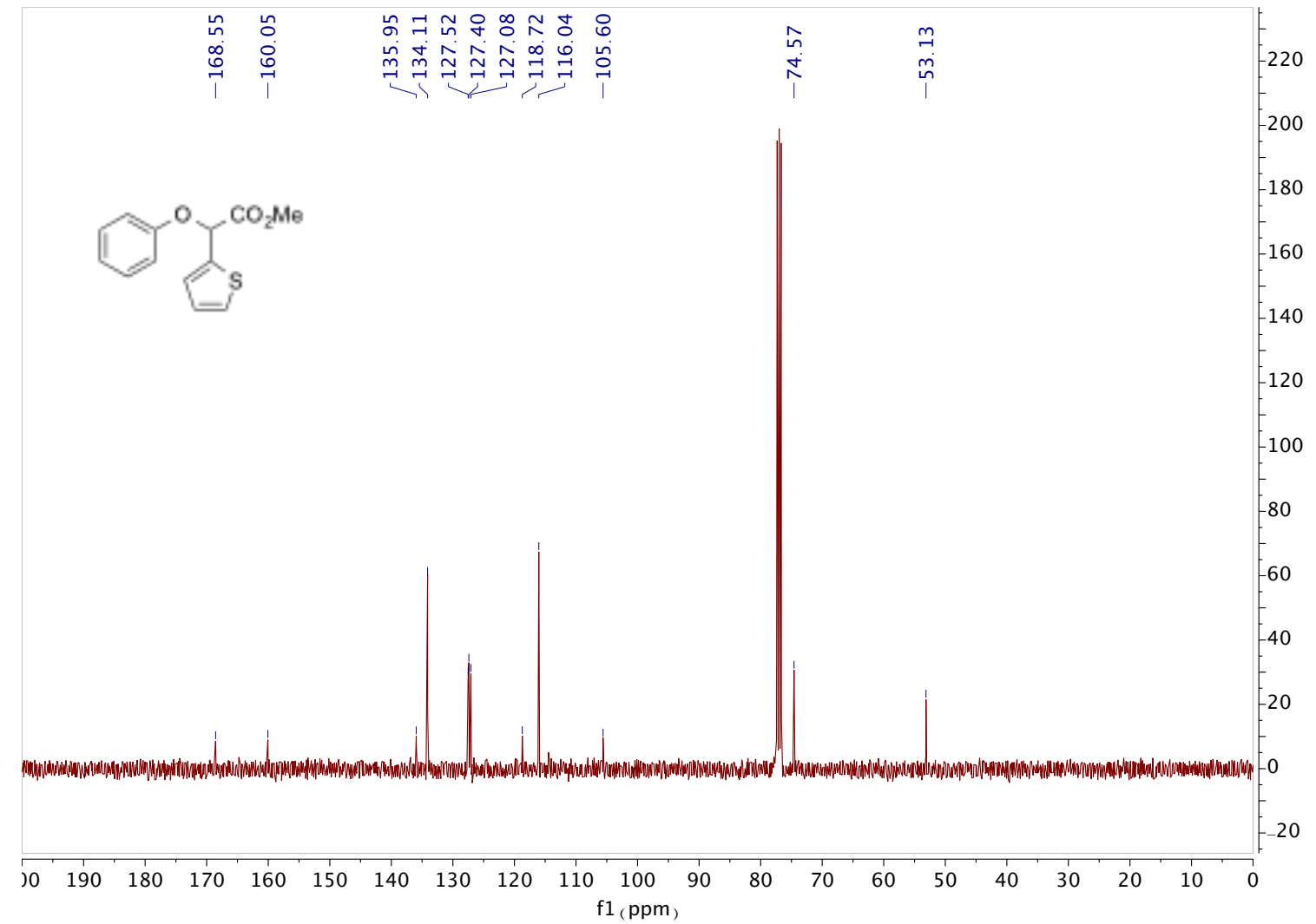


4-(2,2,2-Trifluoro-1-phenylethoxy)benzonitrile (13)

${ }^{1}$ H NMR (600 MHz, Chloroform- $d$ )<smiles>N#Cc1ccc(OC(c2ccccc2)C(F)(F)c2ccc(C#N)cc2)cc1</smiles>

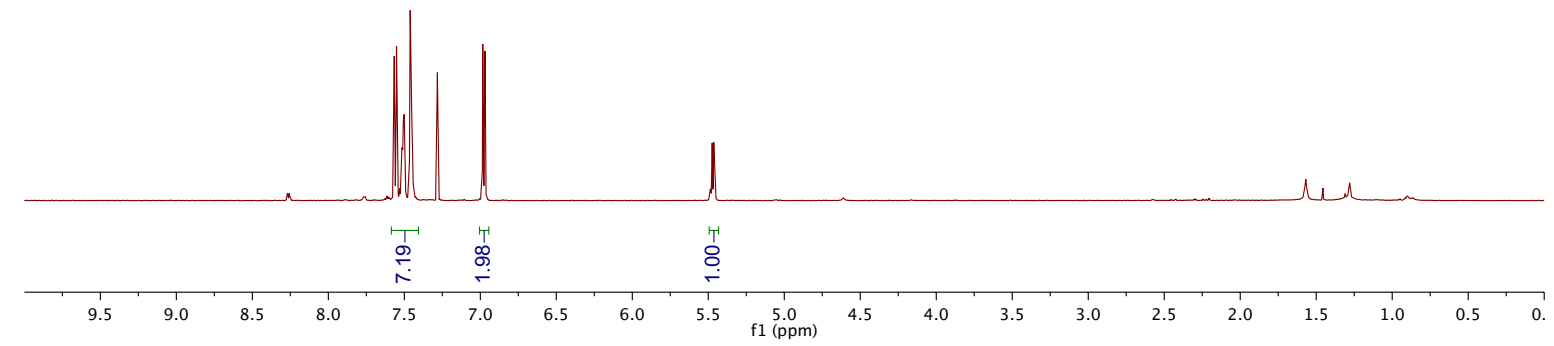

${ }^{13}$ C NMR (151 MHz, Chloroform- $d$ )
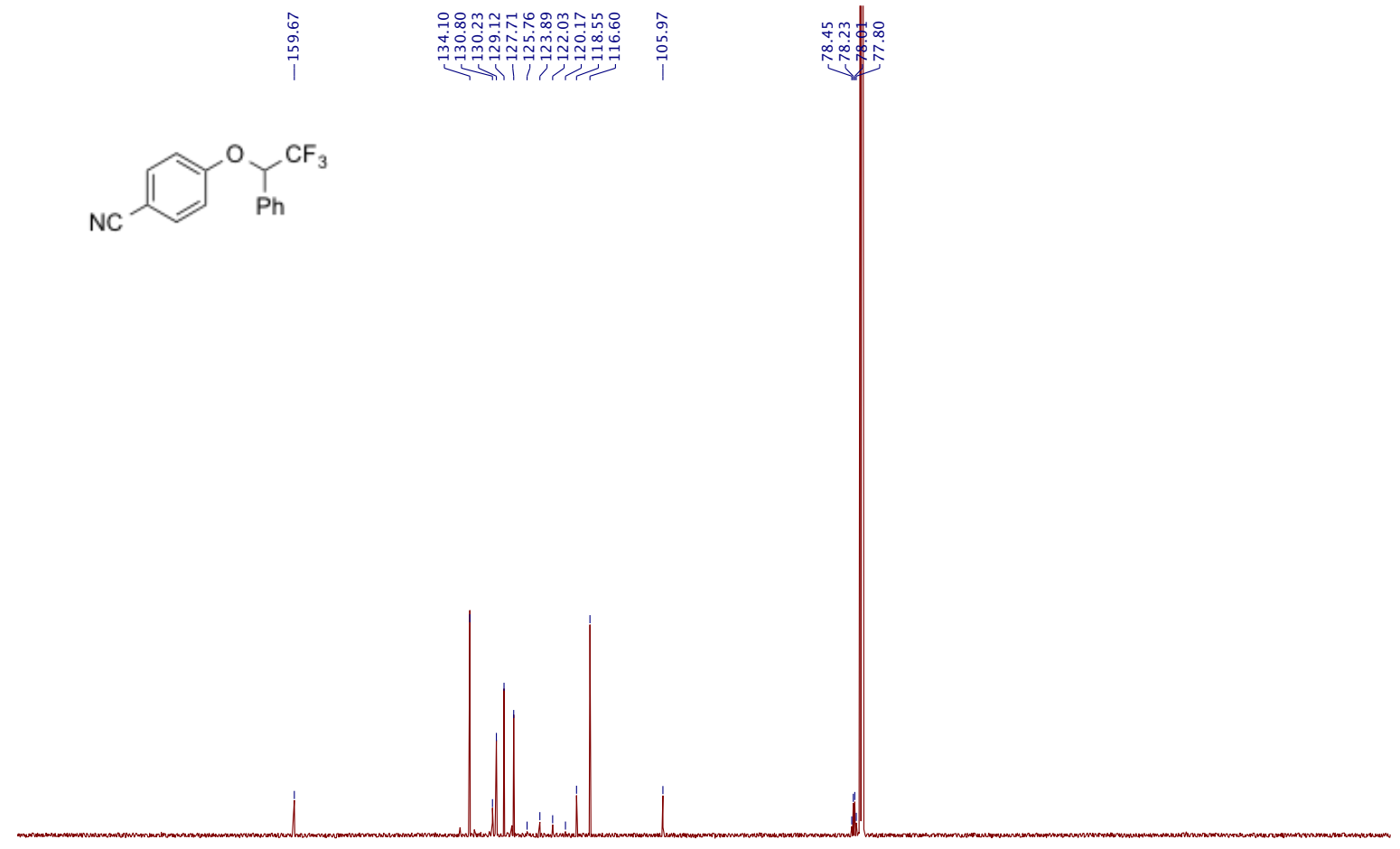

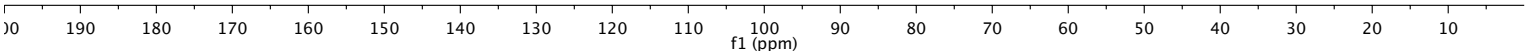


${ }^{19}$ F NMR (565 MHz, Chloroform- $d$ )

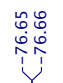<smiles>N#Cc1ccc(OC(c2ccccc2)C(F)(F)F)cc1</smiles>

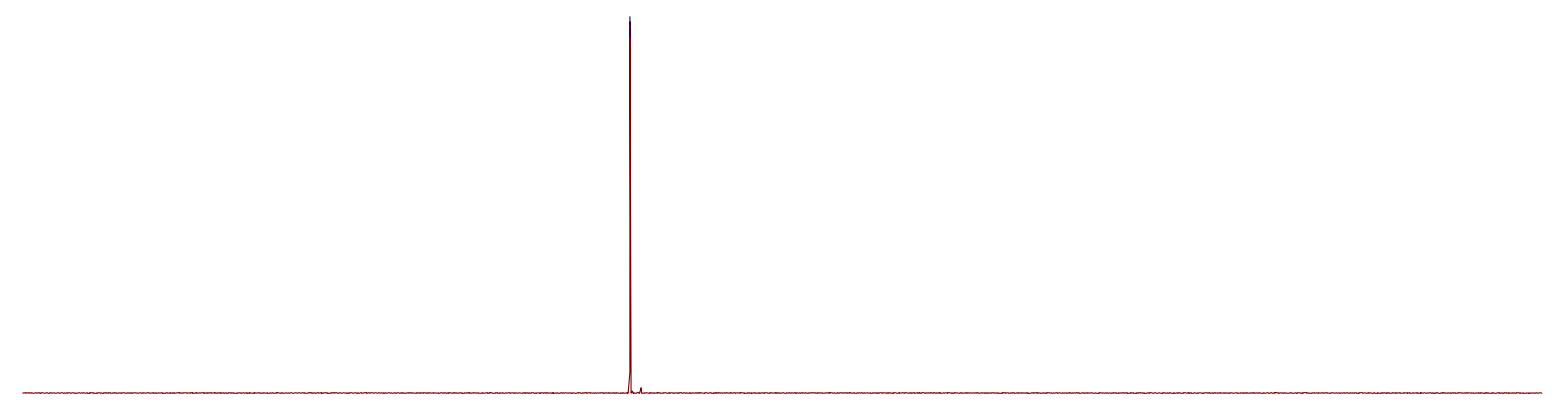

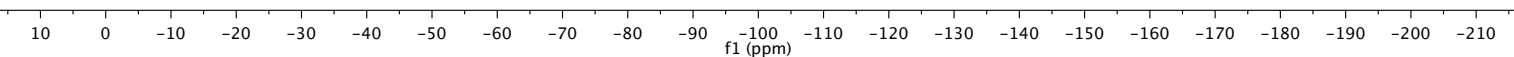


Methyl 2-(4-nitrophenoxy)-2-phenylacetate (15a)

${ }^{1}$ H NMR (400 MHz, Chloroform- $d$ )

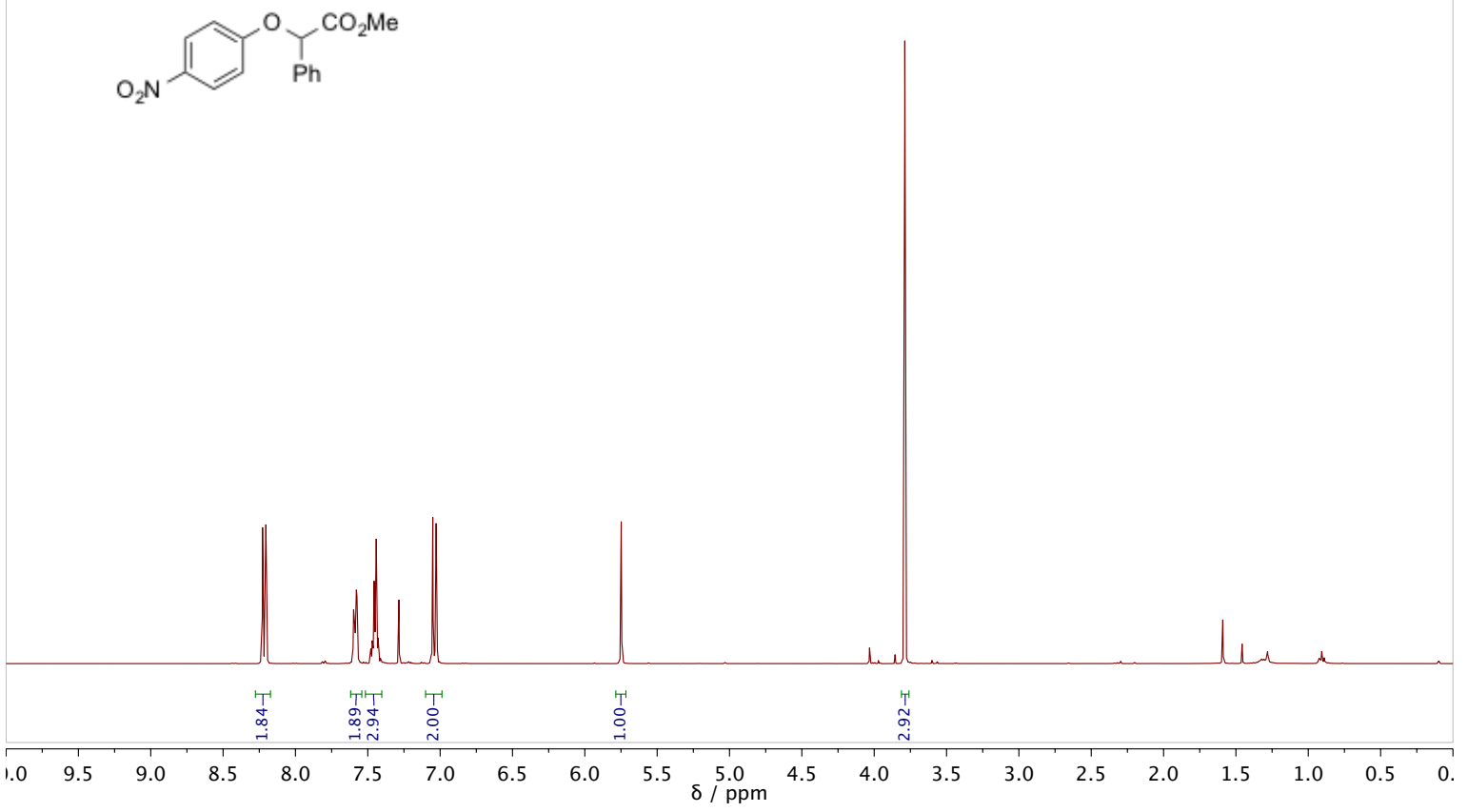

${ }^{13}$ C NMR (101MHz, Chloroform- $d$ )

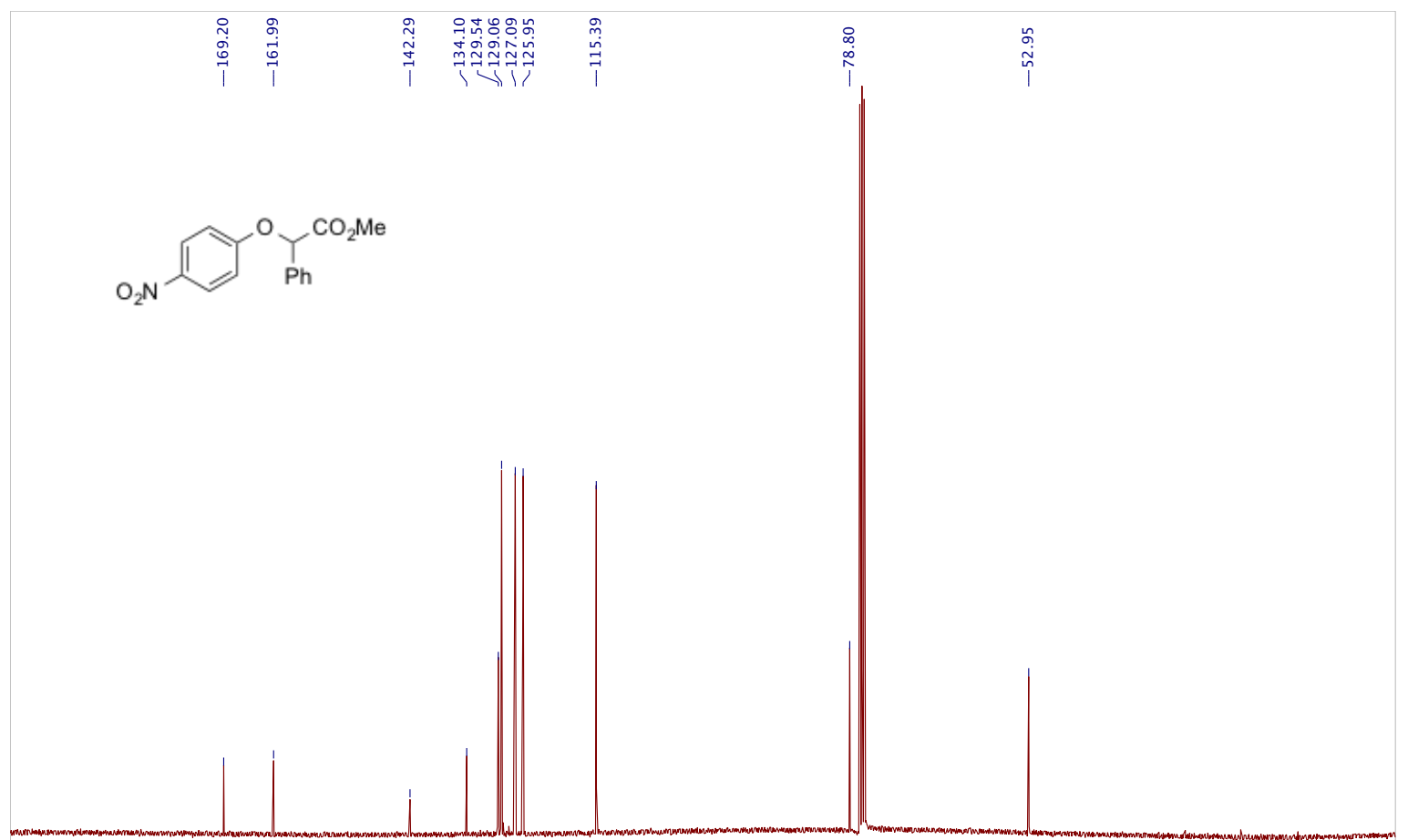

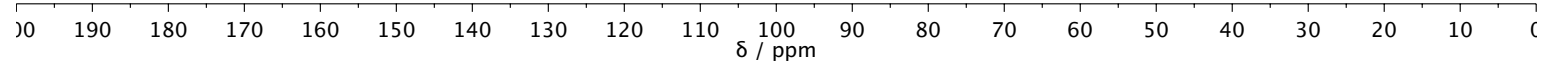


Methyl 2-phenoxy-2-phenylacetate (15b)

${ }^{1}$ H NMR (400 MHz, Chloroform- $d$ )

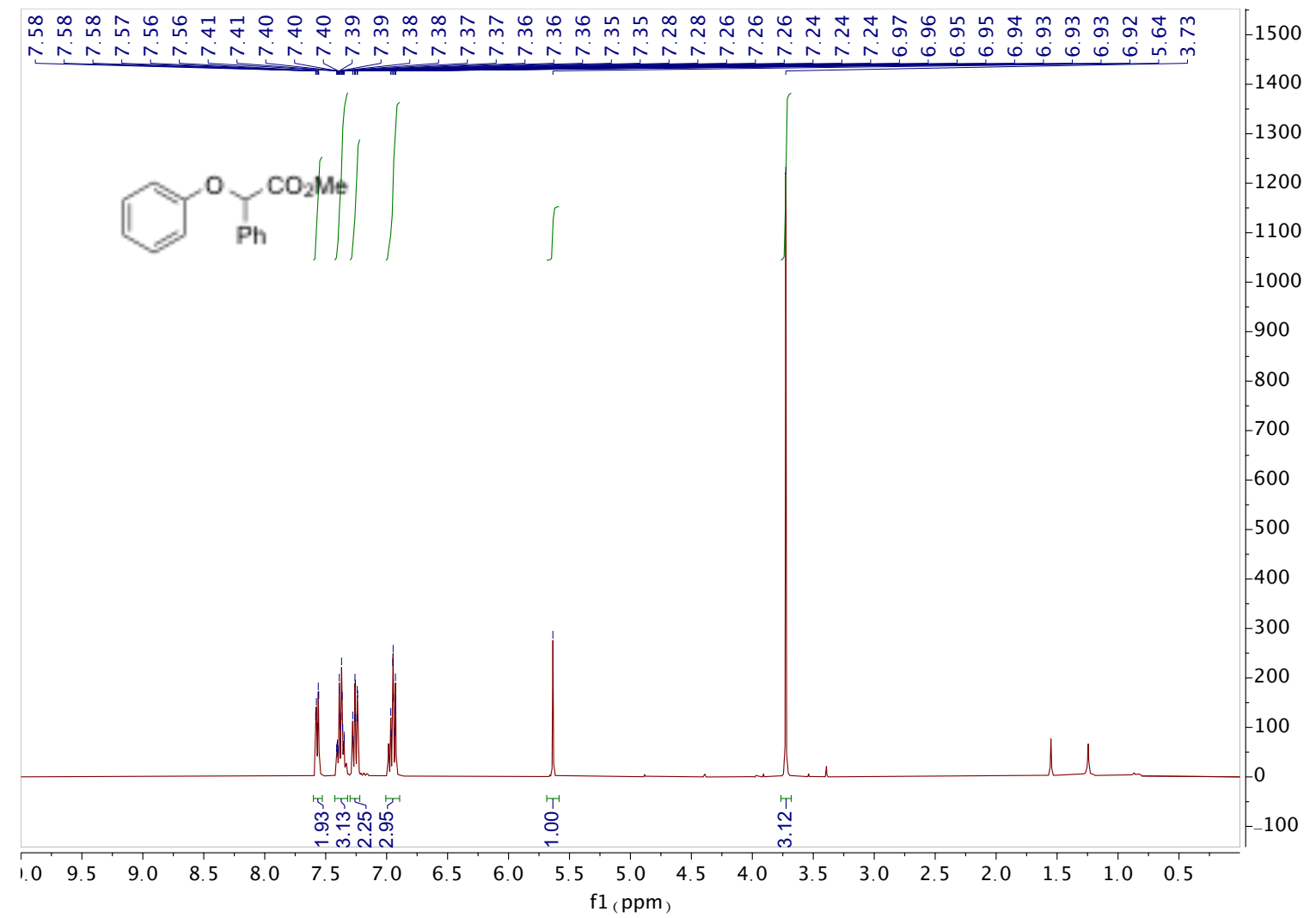

${ }^{13}$ C NMR (101 MHz, Chloroform- $d$ )

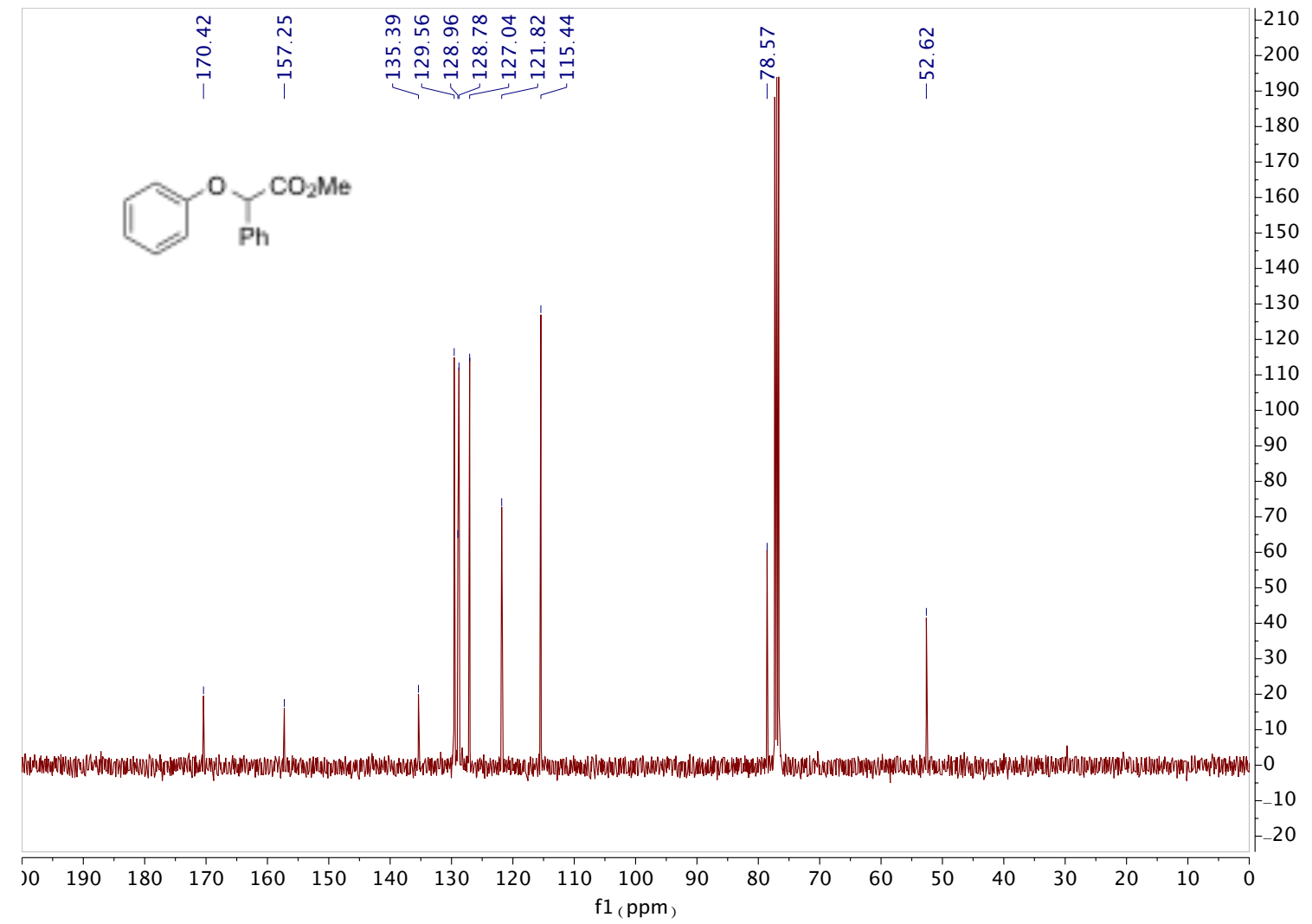


Methyl 2-(4-fluorophenoxy)-2-phenylacetate (15c)

${ }^{1}$ H NMR (600 MHz, Chloroform- $d$ )<smiles>CC(=O)C(Oc1ccc(F)cc1)c1ccccc1</smiles>

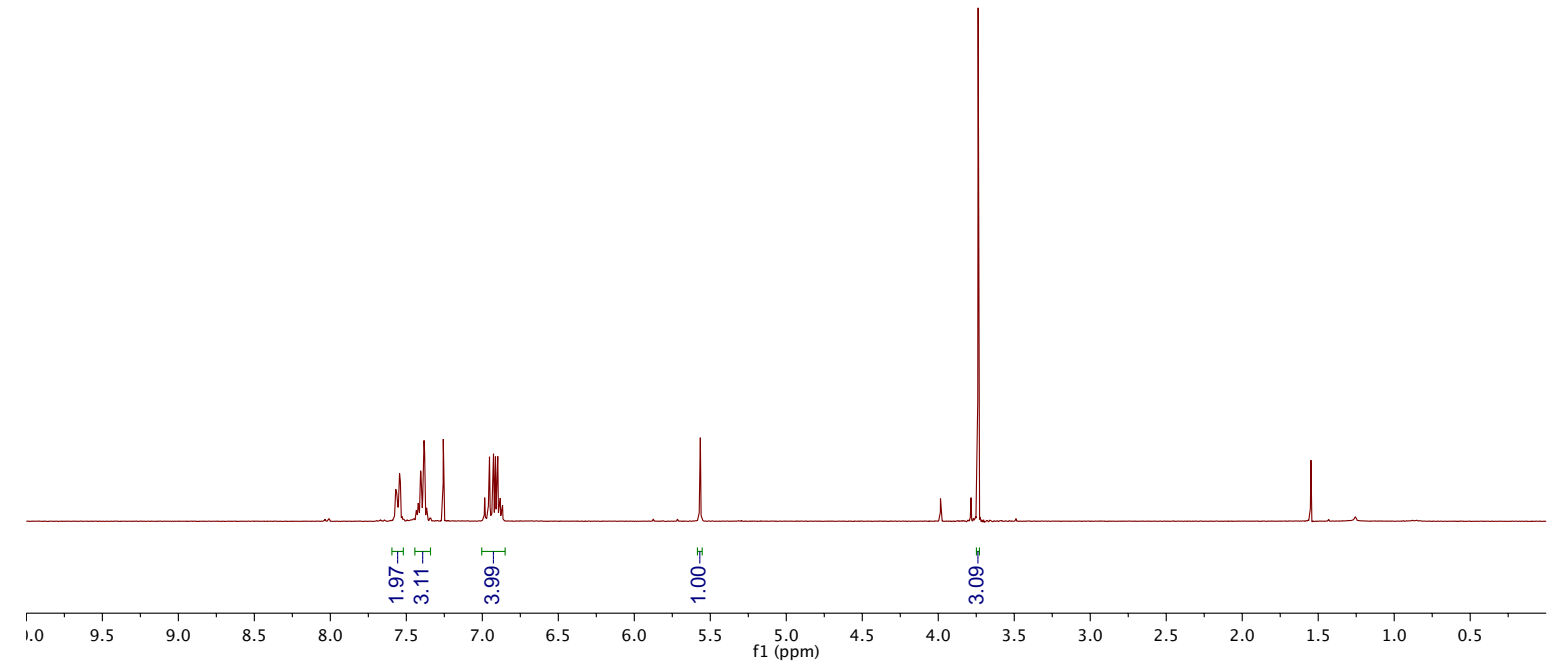

${ }^{13}$ C NMR (151 MHz, Chloroform- $d$ )
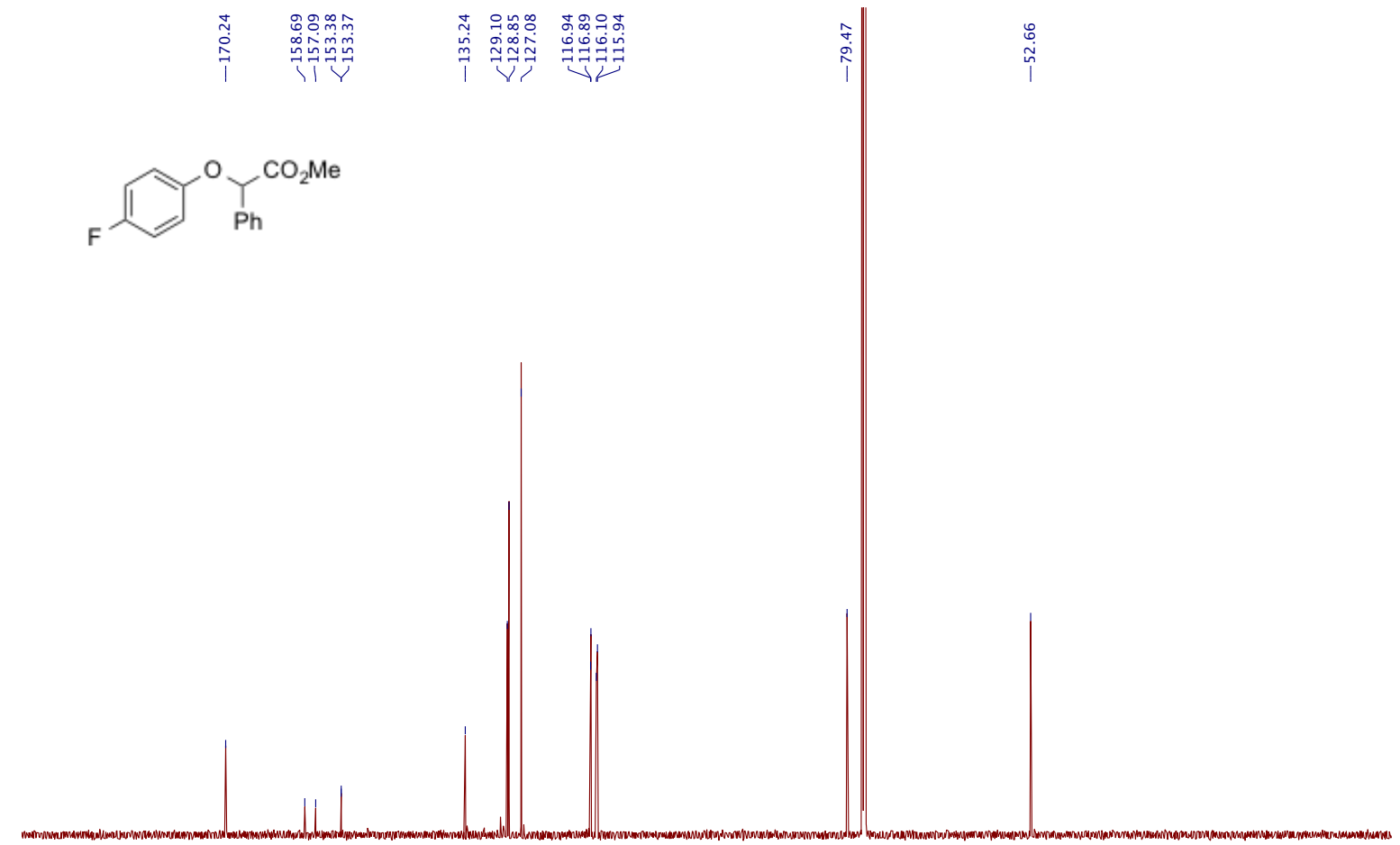

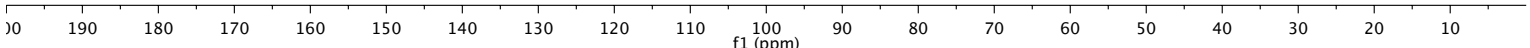


${ }^{19}$ F NMR (565 MHz, Chloroform-d)

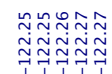

$\overbrace{\mathrm{Ph}}^{\mathrm{O}} \mathrm{YO}_{2}^{\mathrm{CO}_{2} \mathrm{Me}}$

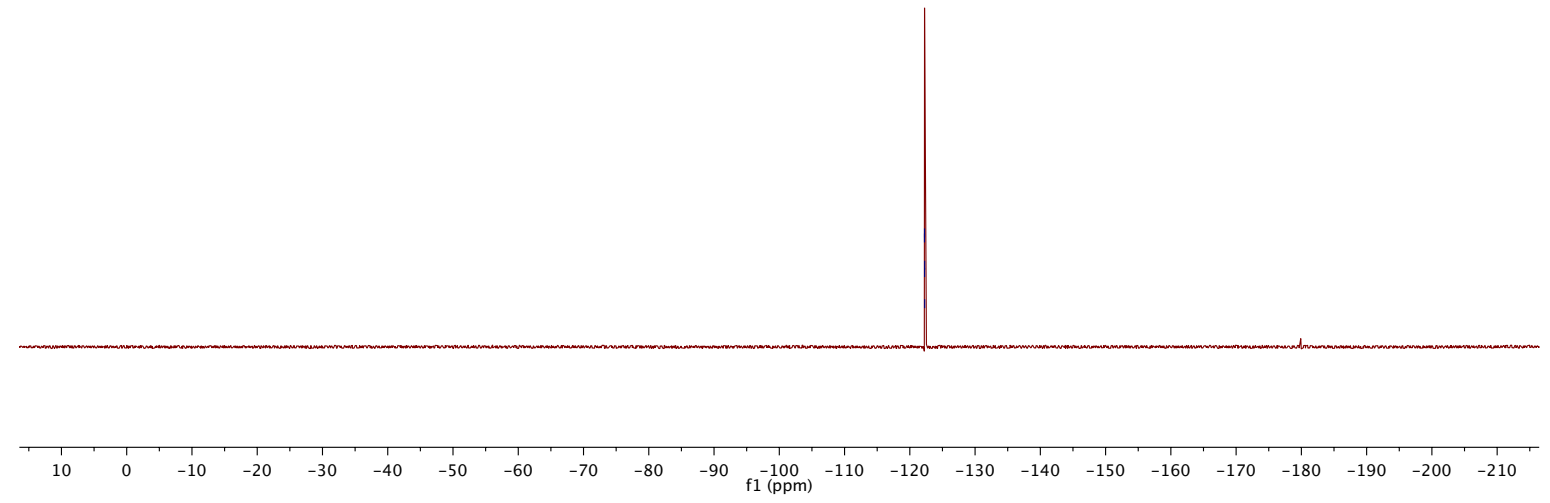


Methyl 2-(4-chlorophenoxy)-2-phenylacetate (15d)

${ }^{1}$ H NMR $(600 \mathrm{MHz}$, Chloroform- $d)$

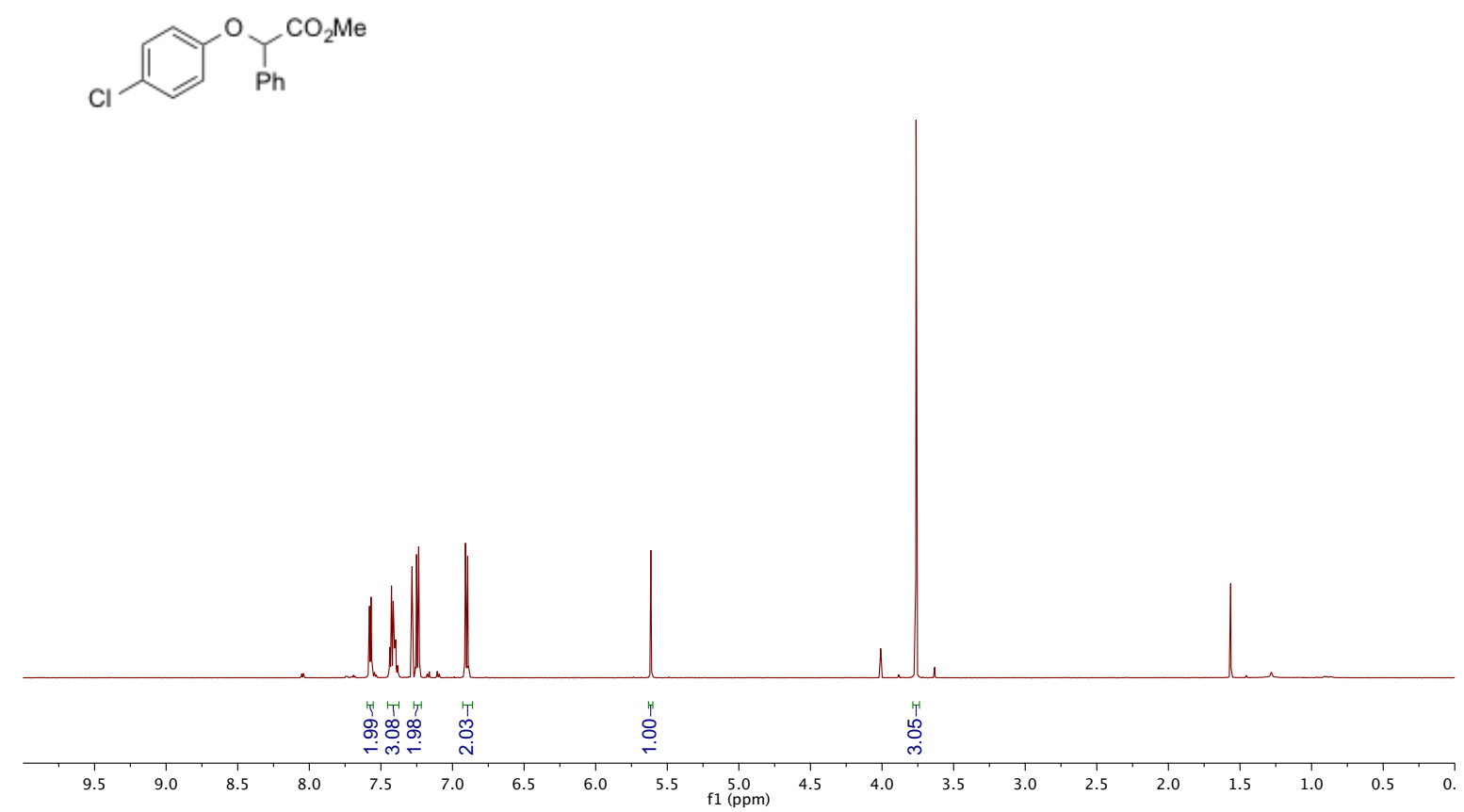

${ }^{13}$ C NMR (151 MHz, Chloroform- $d$ )

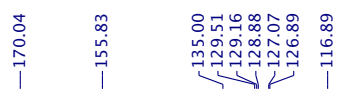

$\overbrace{\mathrm{Cl}}^{\mathrm{O}} \mathrm{Y}_{\mathrm{Ph}}^{\mathrm{CO}_{2} \mathrm{Me}}$
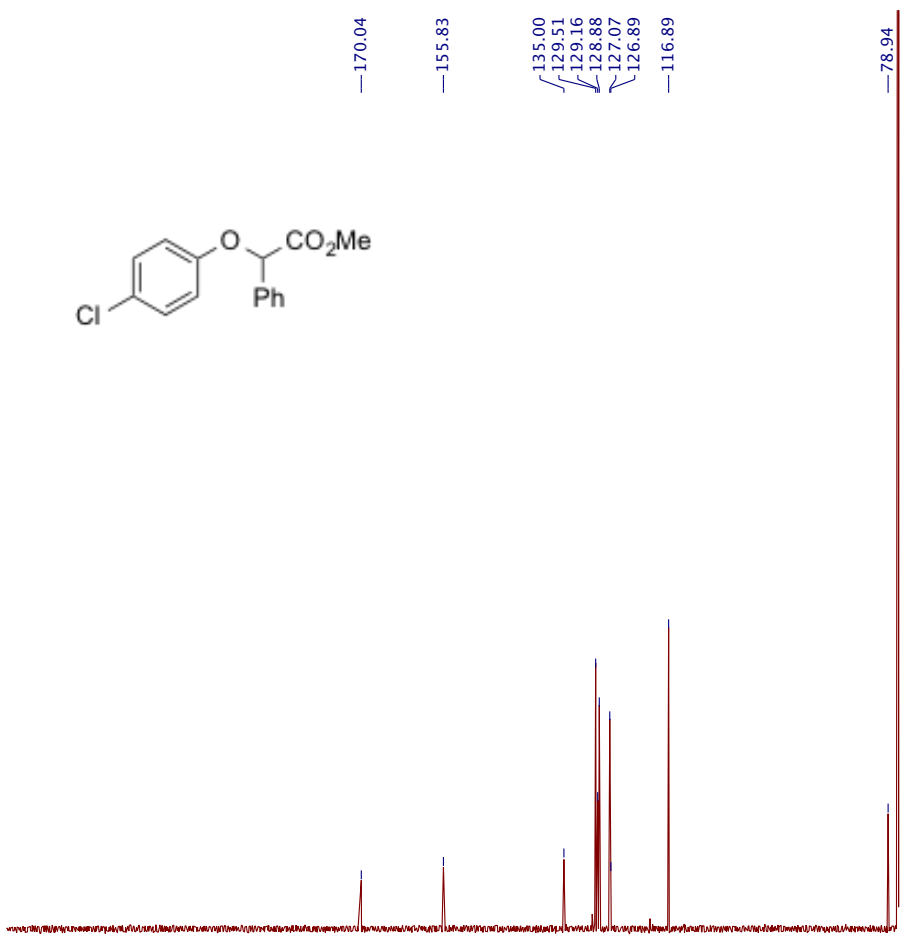

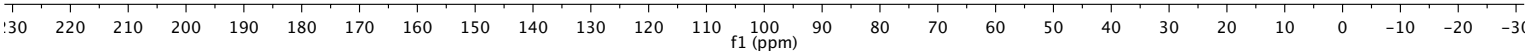


Methyl 2-phenyl-2-(4-(trifluoromethoxy)phenoxy)acetate (15e)

${ }^{1}$ H NMR (600 MHz, Chloroform- $\left.d\right)$

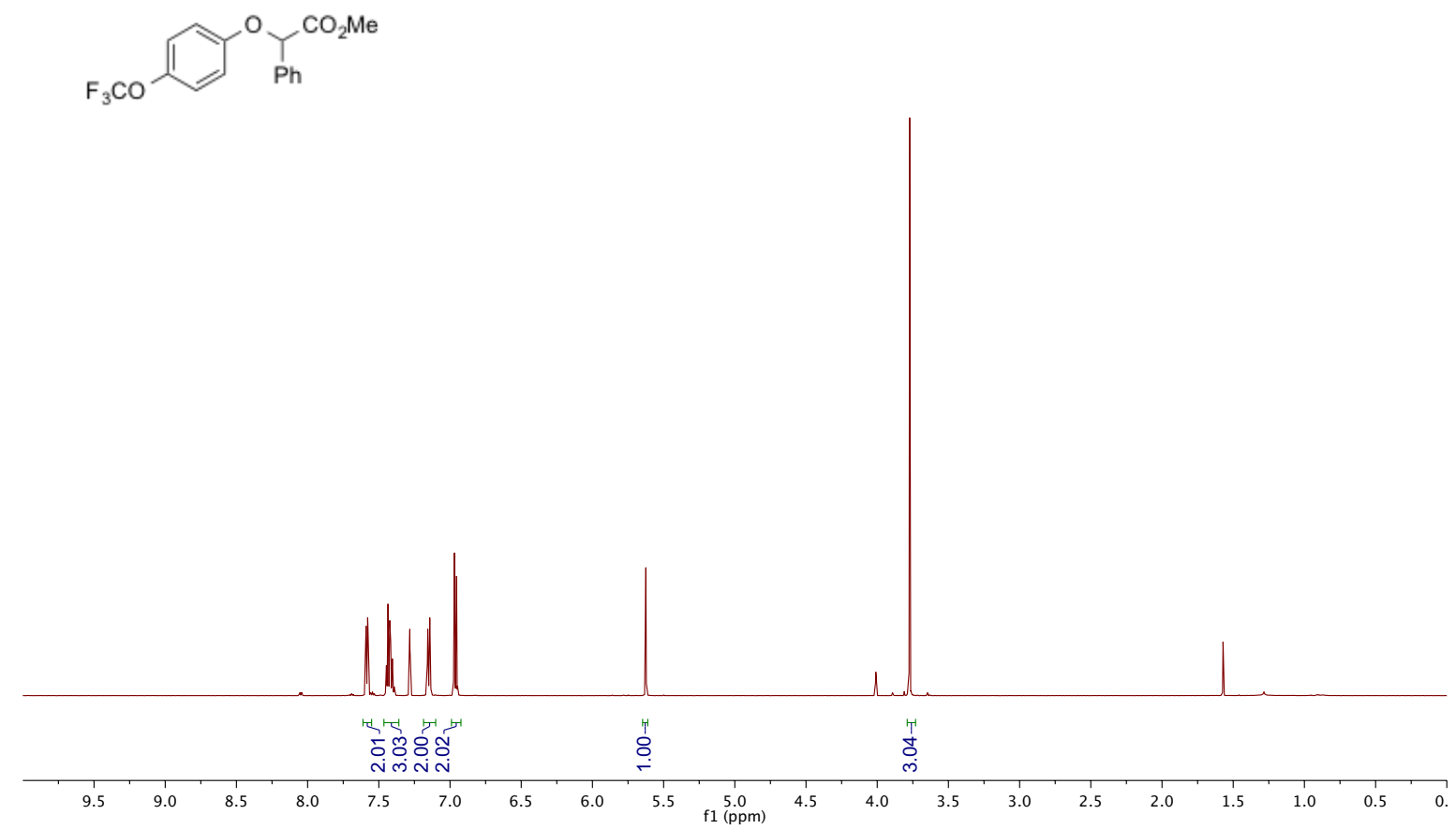

${ }^{13}$ C NMR (151 MHz, Chloroform- $d$ )

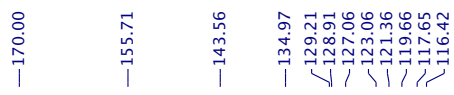

${ }_{\mathrm{F}_{3} \mathrm{CO}}^{\mathrm{O}} \mathrm{Y}_{\mathrm{Ph}}^{\mathrm{CO}_{2} \mathrm{Me}}$

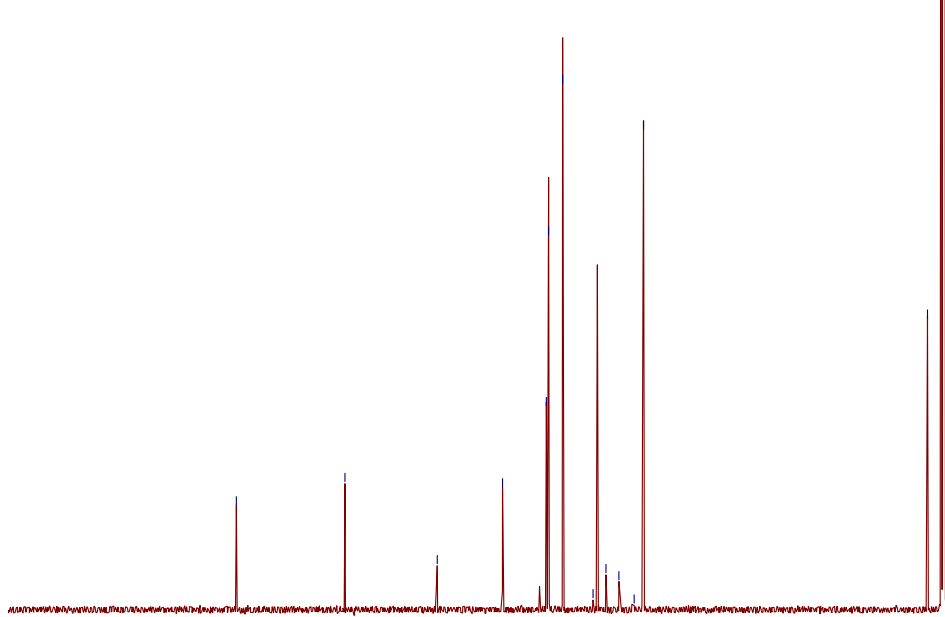

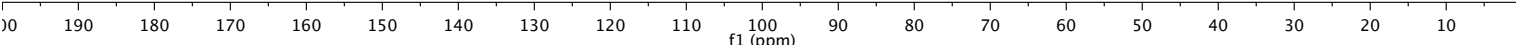


${ }^{19}$ F NMR (565 MHz, Chloroform- $d$ )

$$
\underset{\substack{m \\ \infty}}{\infty}
$$

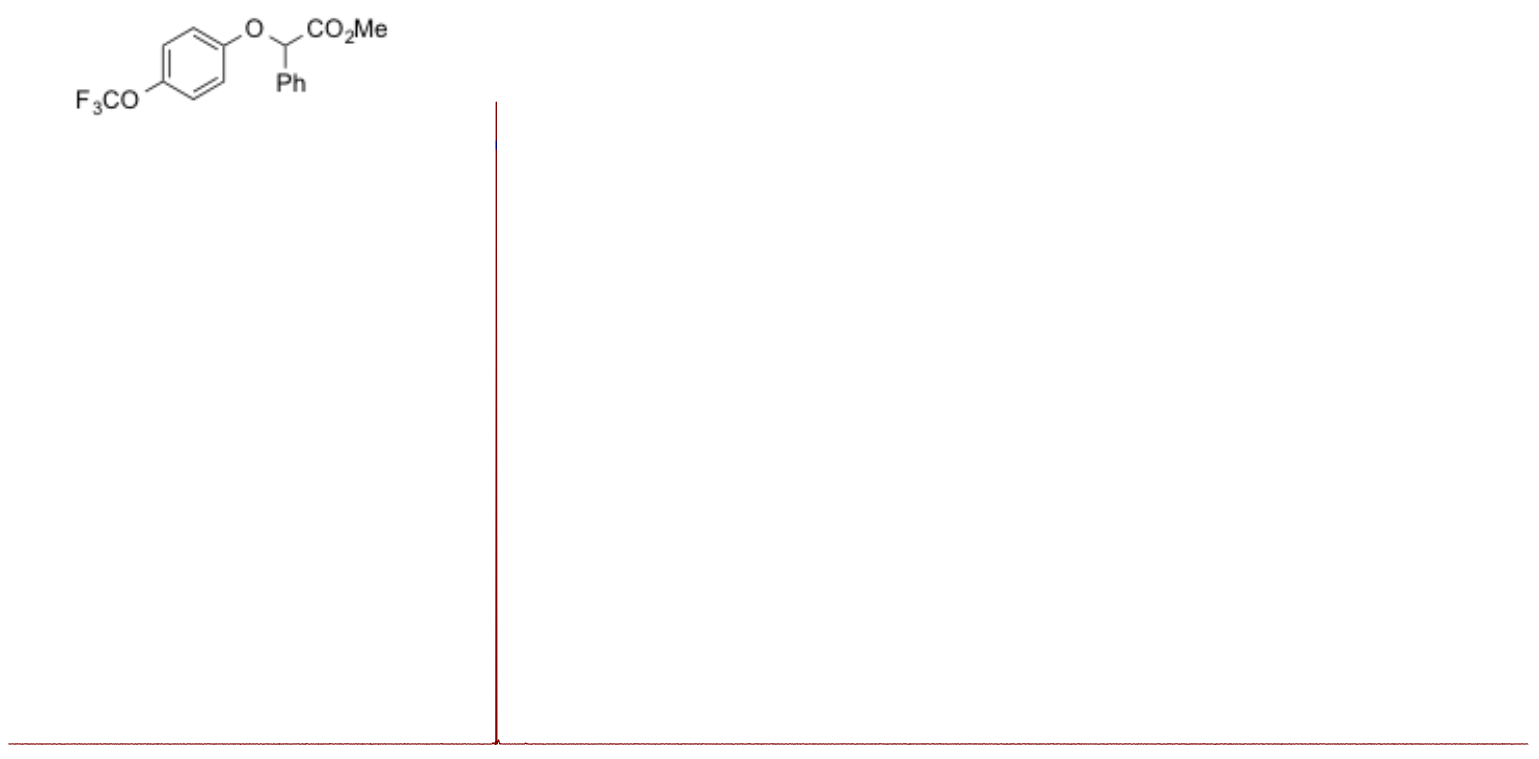

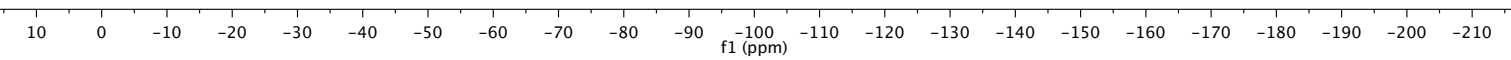


Methyl 2-phenyl-2-(4-(trifluoromethyl)phenoxy)acetate (15f)

${ }^{1}$ H NMR (600 MHz, Chloroform- $\left.d\right)$

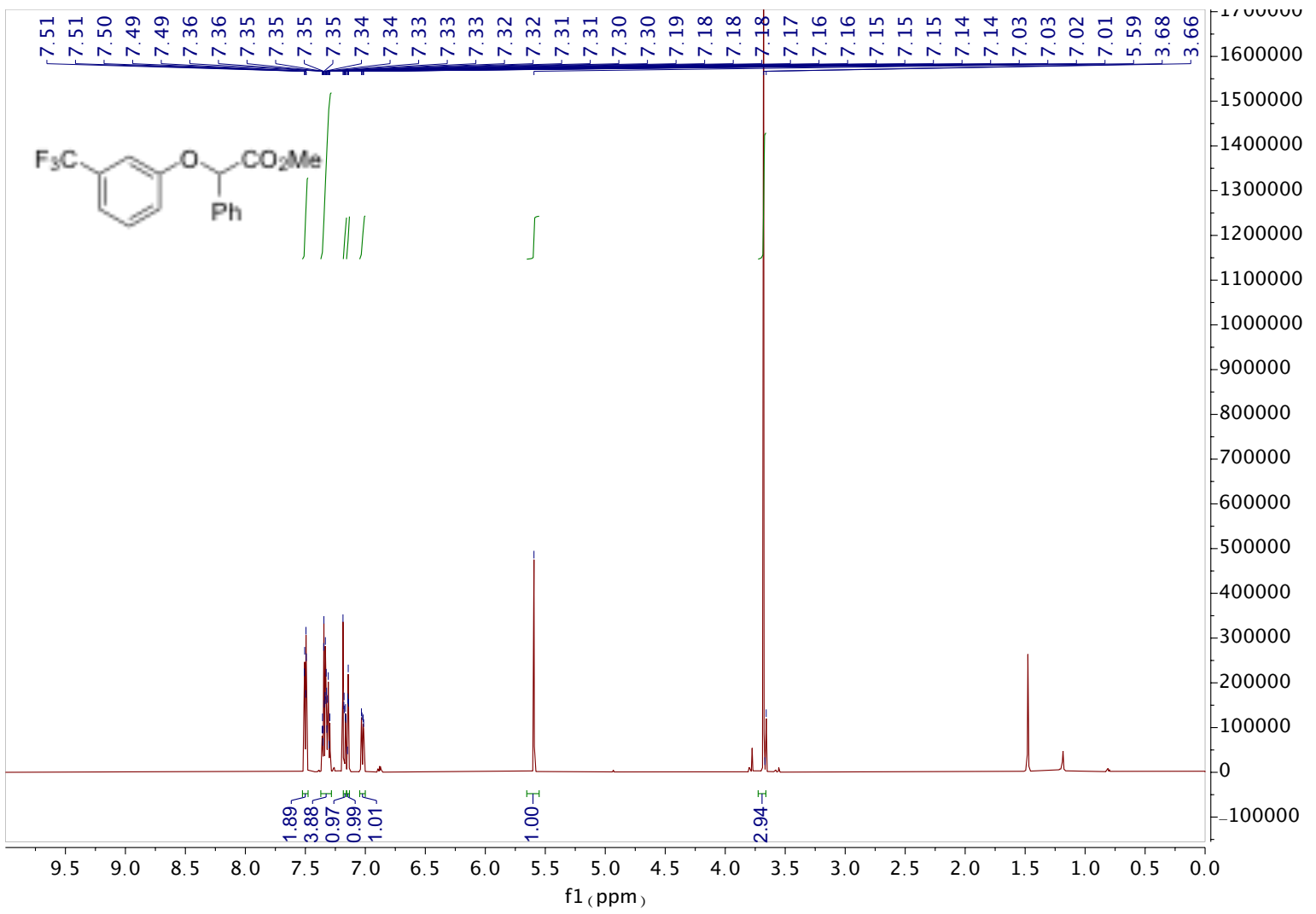

${ }^{13}$ C NMR (151MHz, Chloroform- $d$ )

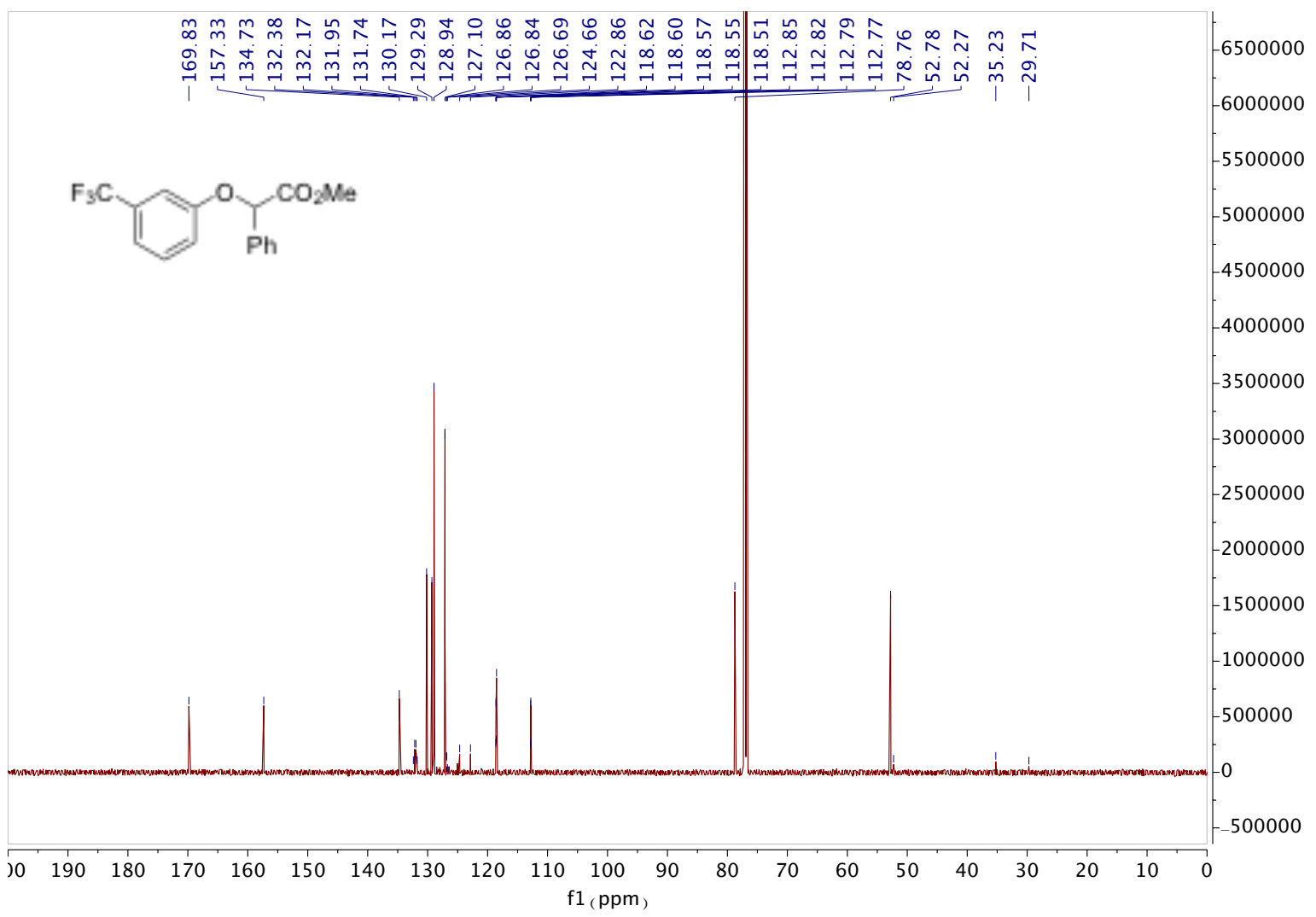


${ }^{19}$ F NMR (565 MHz, Chloroform- $d$ )

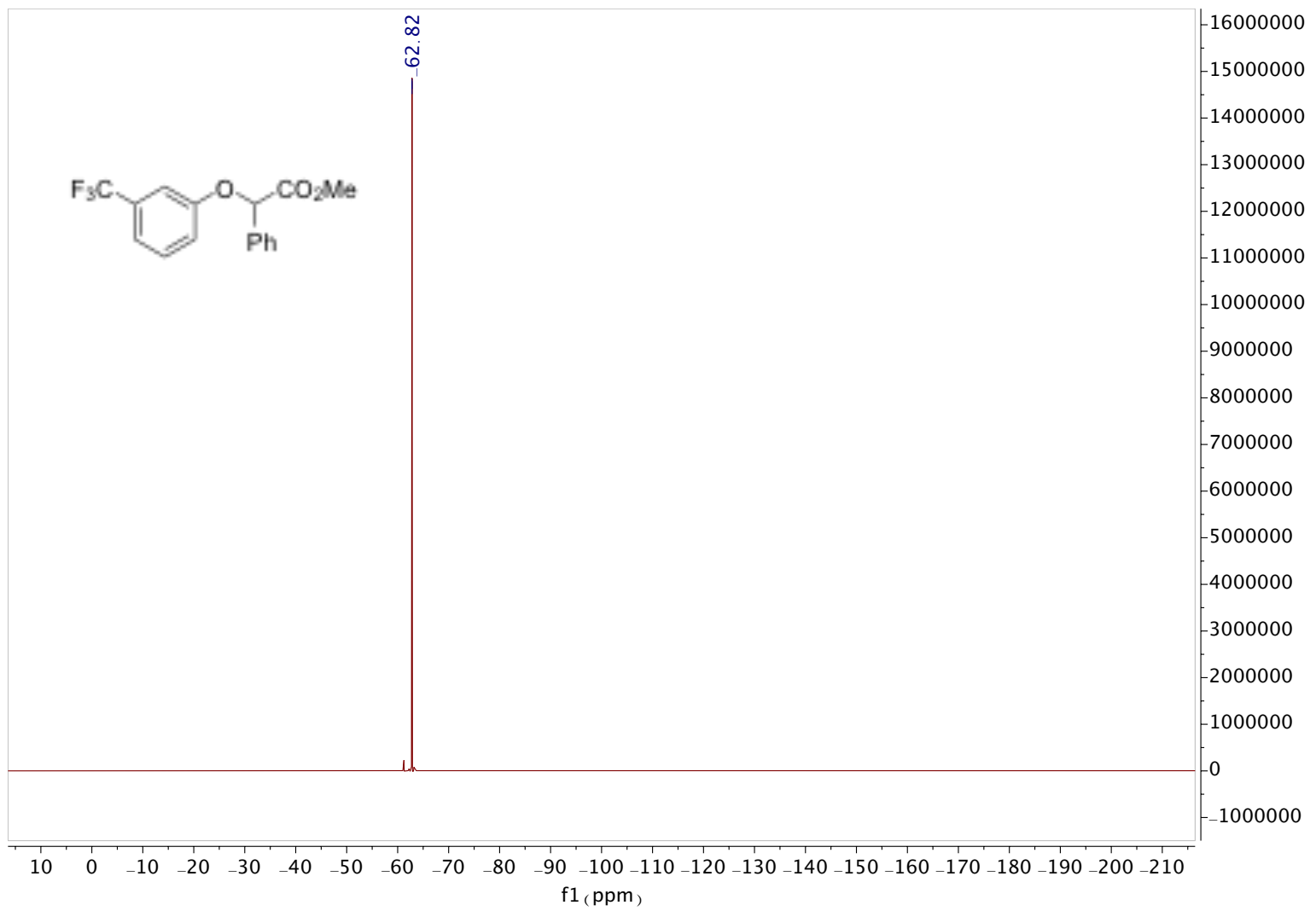


Methyl 4-(2-methoxy-2-oxo-1-phenylethoxy)benzoate (15g)

${ }^{1}$ H NMR (600 MHz, Chloroform- $d$ )

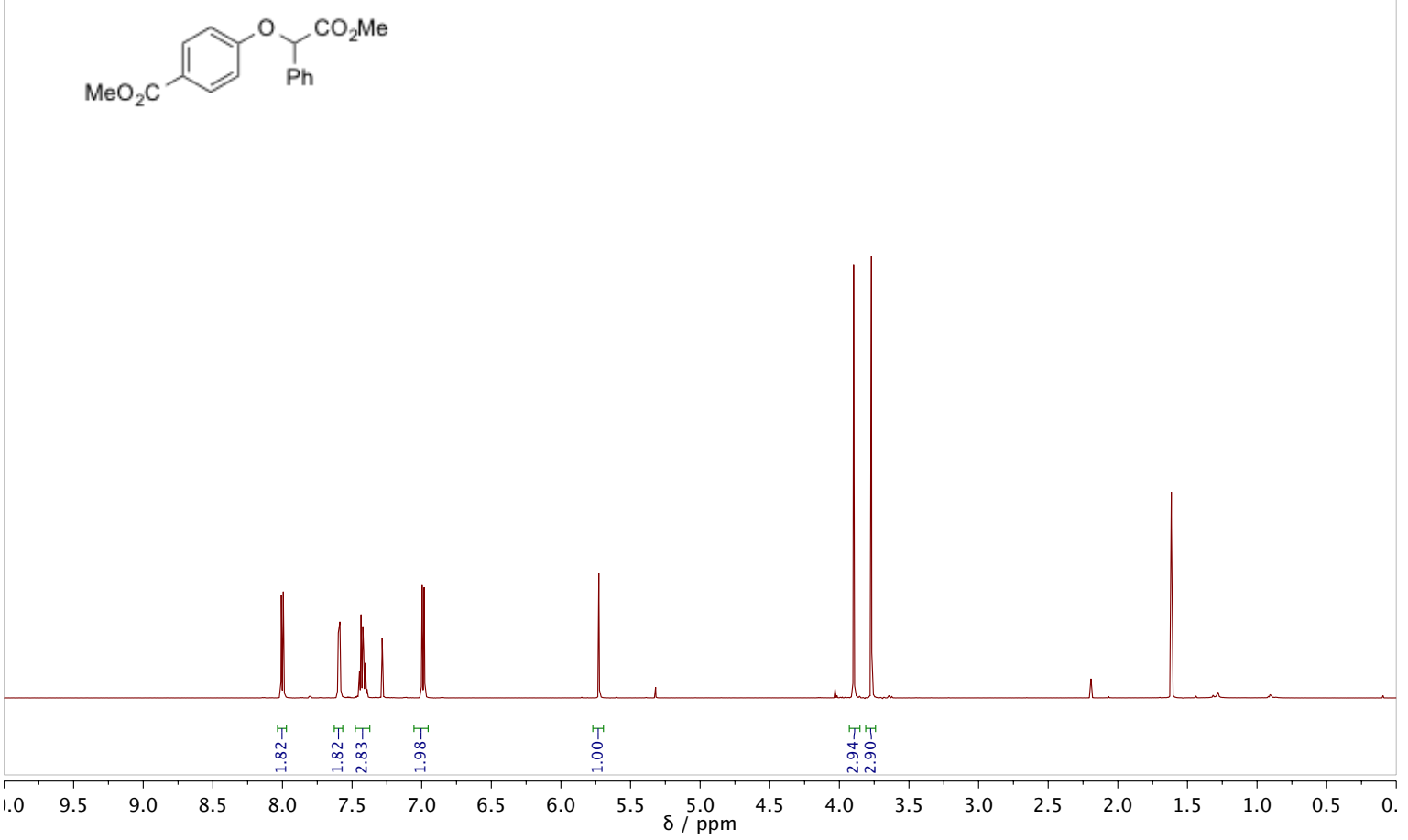

${ }^{13}$ C NMR (151 MHz, Chloroform- $d$ )

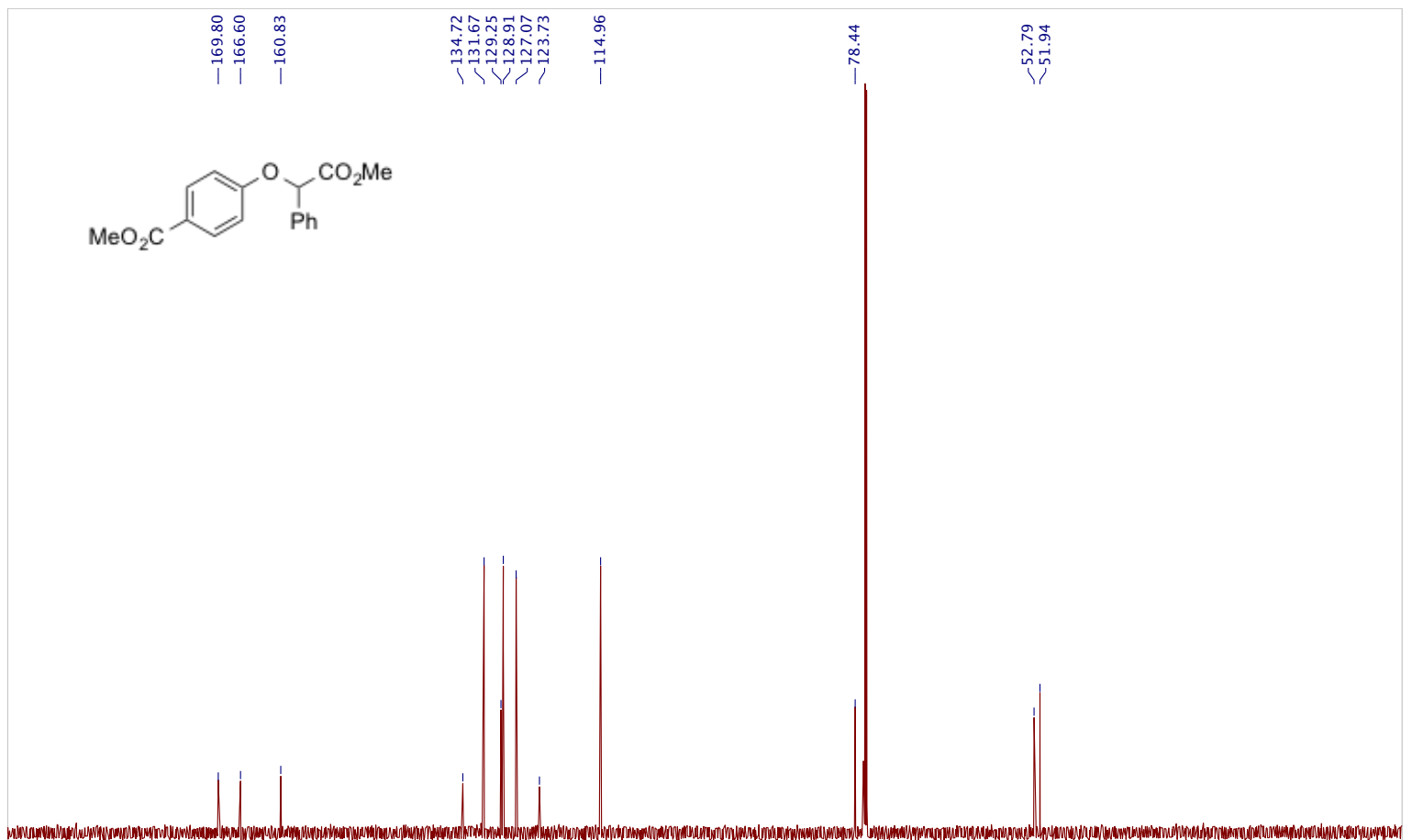

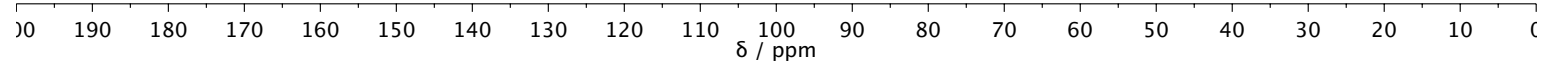


Methyl 2-(3-nitrophenoxy)-2-phenylacetate (15h)

${ }^{1}$ H NMR (600 MHz, Chloroform- $\left.d\right)$<smiles>CC(=O)C(Oc1cccc([N+](=O)[O-])c1)c1ccccc1</smiles>

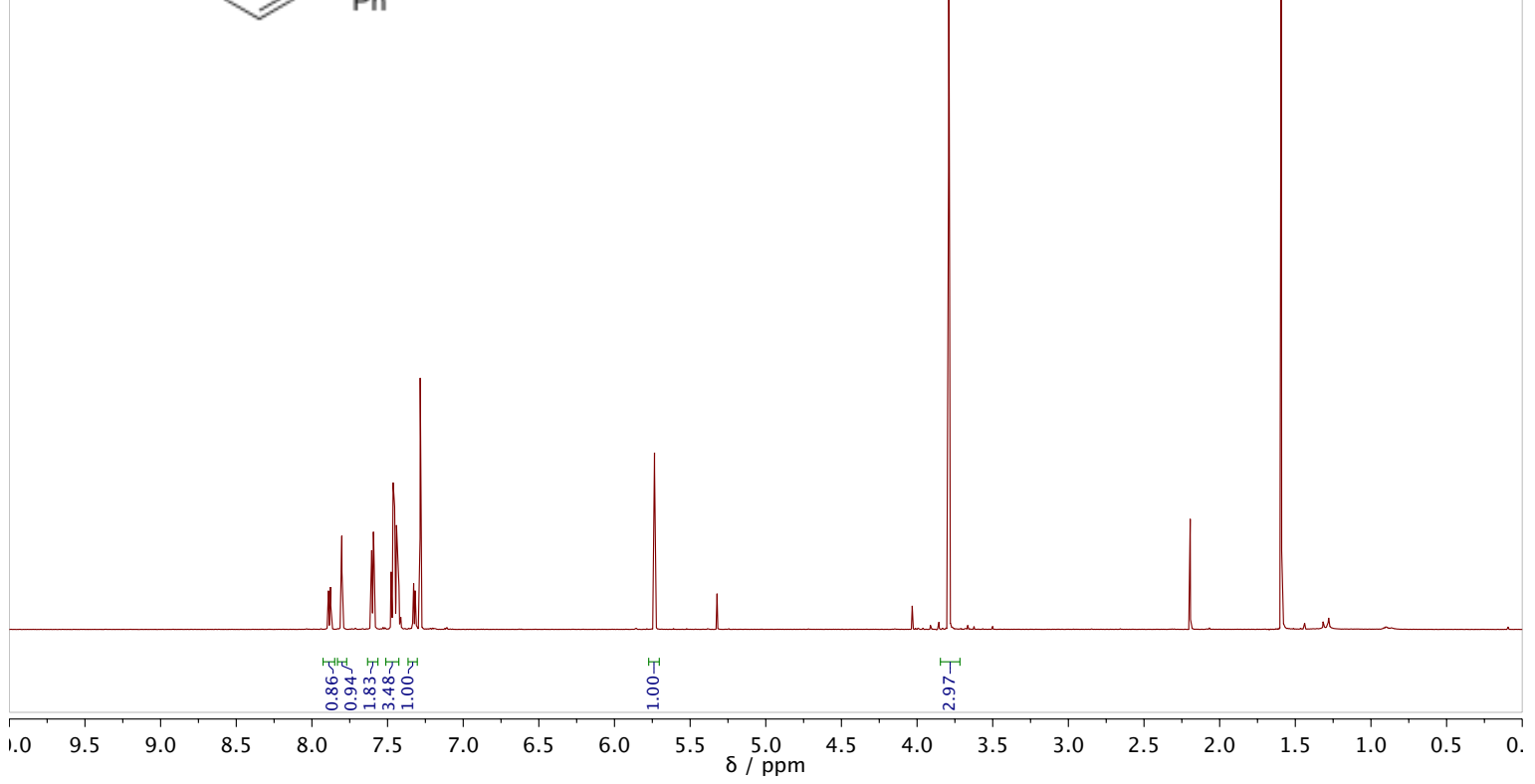

${ }^{13}$ C NMR (151 MHz, Chloroform- $d$ )

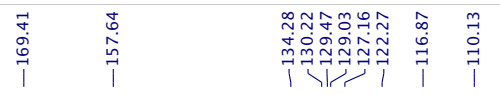

$$
\begin{aligned}
& \mathrm{O}_{2} \mathrm{~N} \mathrm{Y}_{\mathrm{Ph}}^{\mathrm{O}} \mathrm{CO}_{2} \mathrm{Me}
\end{aligned}
$$

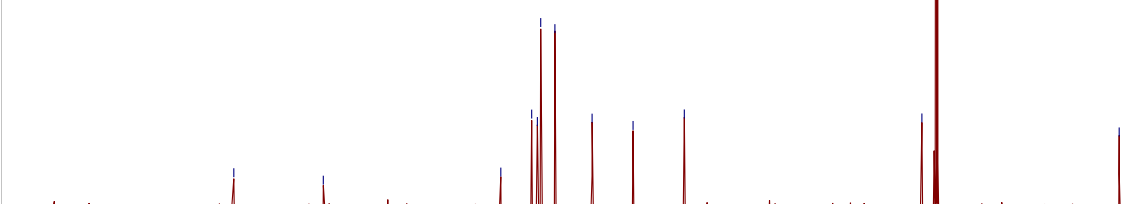


Methyl 2-(3-fluorophenoxy)-2-phenylacetate (15i)

${ }^{1}$ H NMR (600 MHz, Chloroform- $d$ )

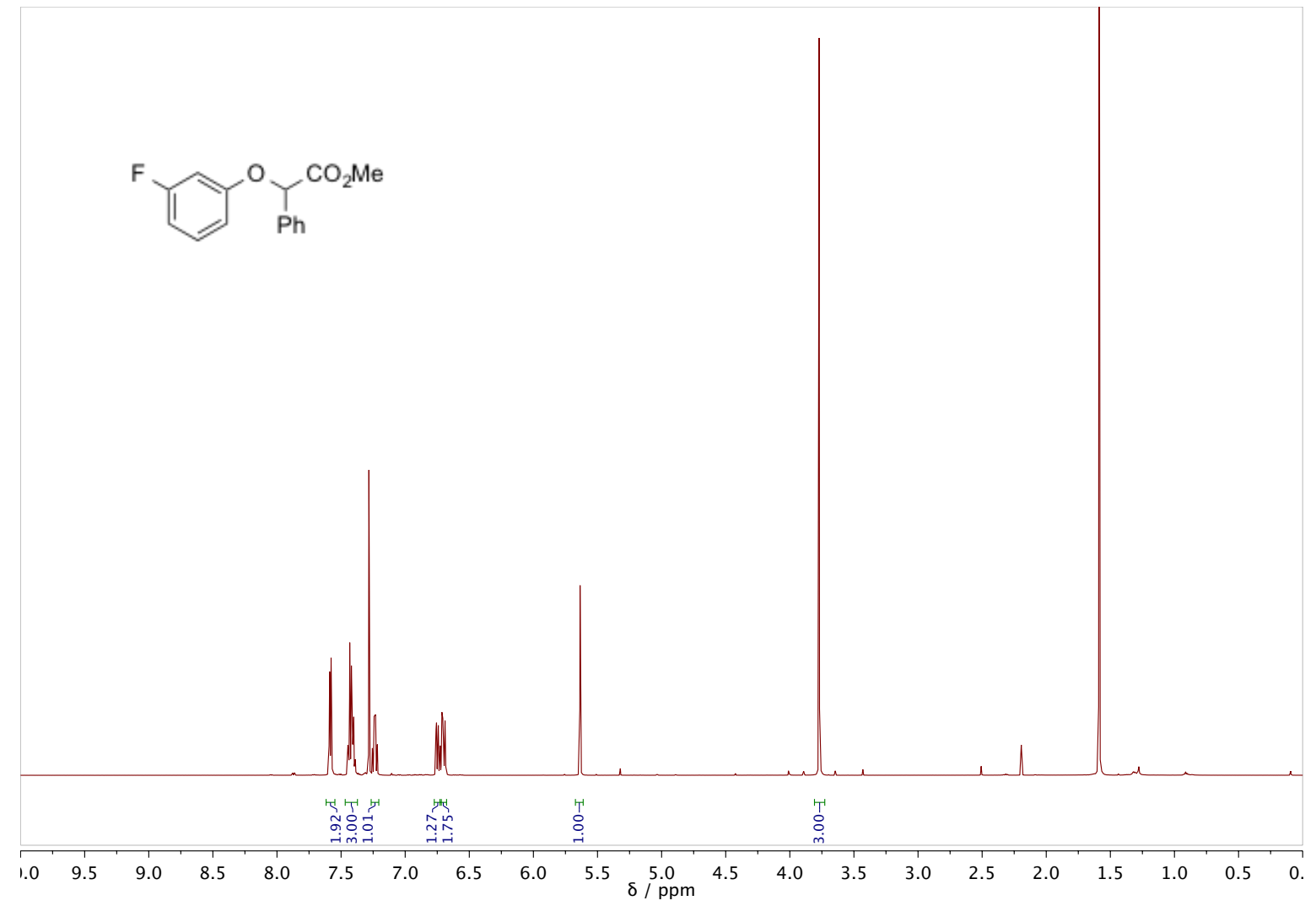

${ }^{13}$ C NMR (151 MHz, Chloroform- $d$ )
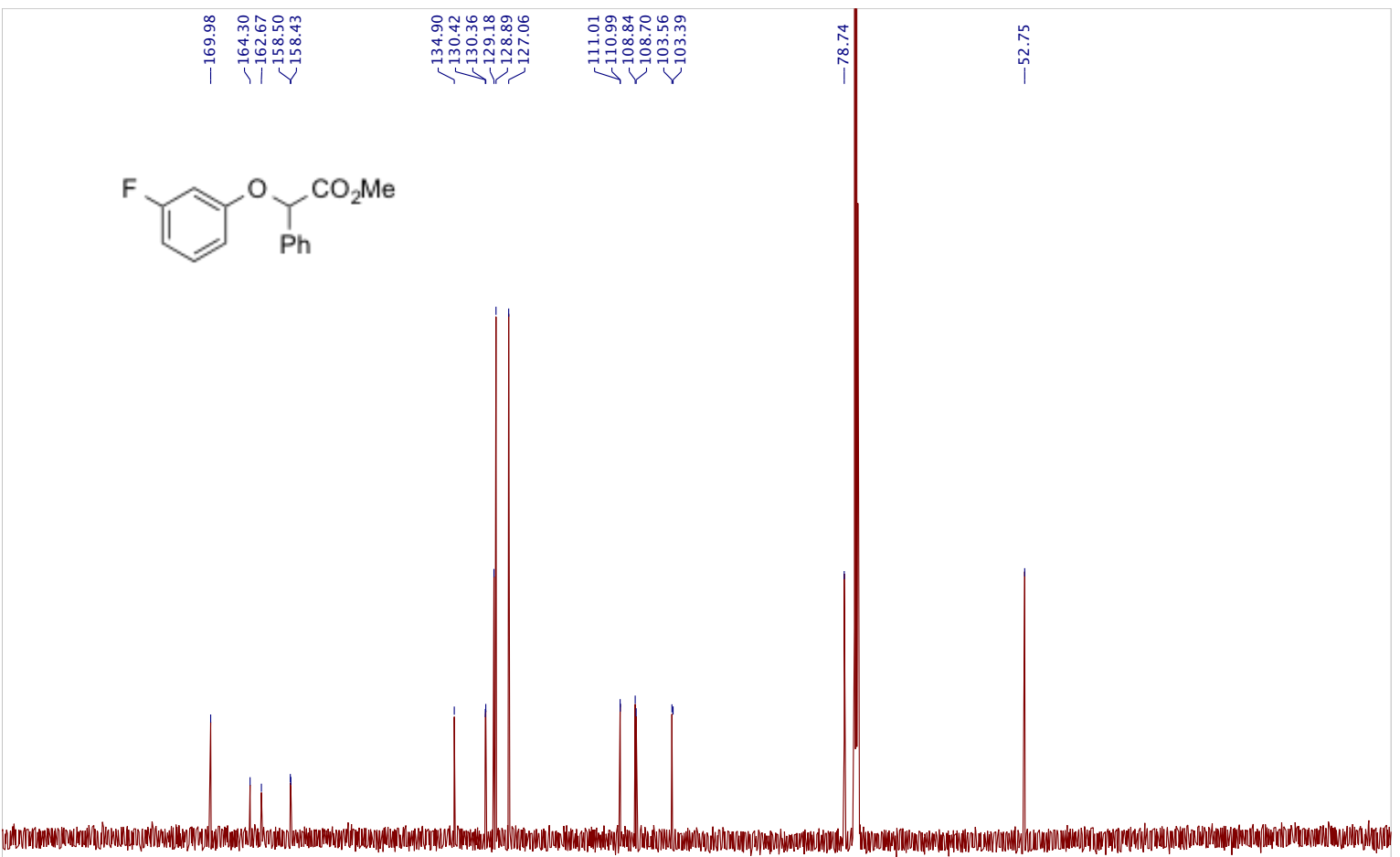

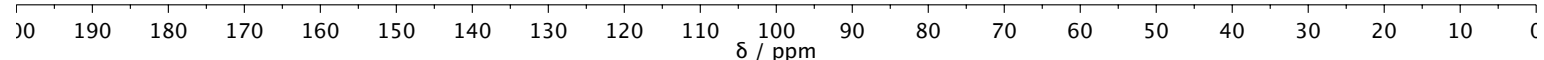


${ }^{19}$ F NMR (565 MHz, Chloroform- $d$ )

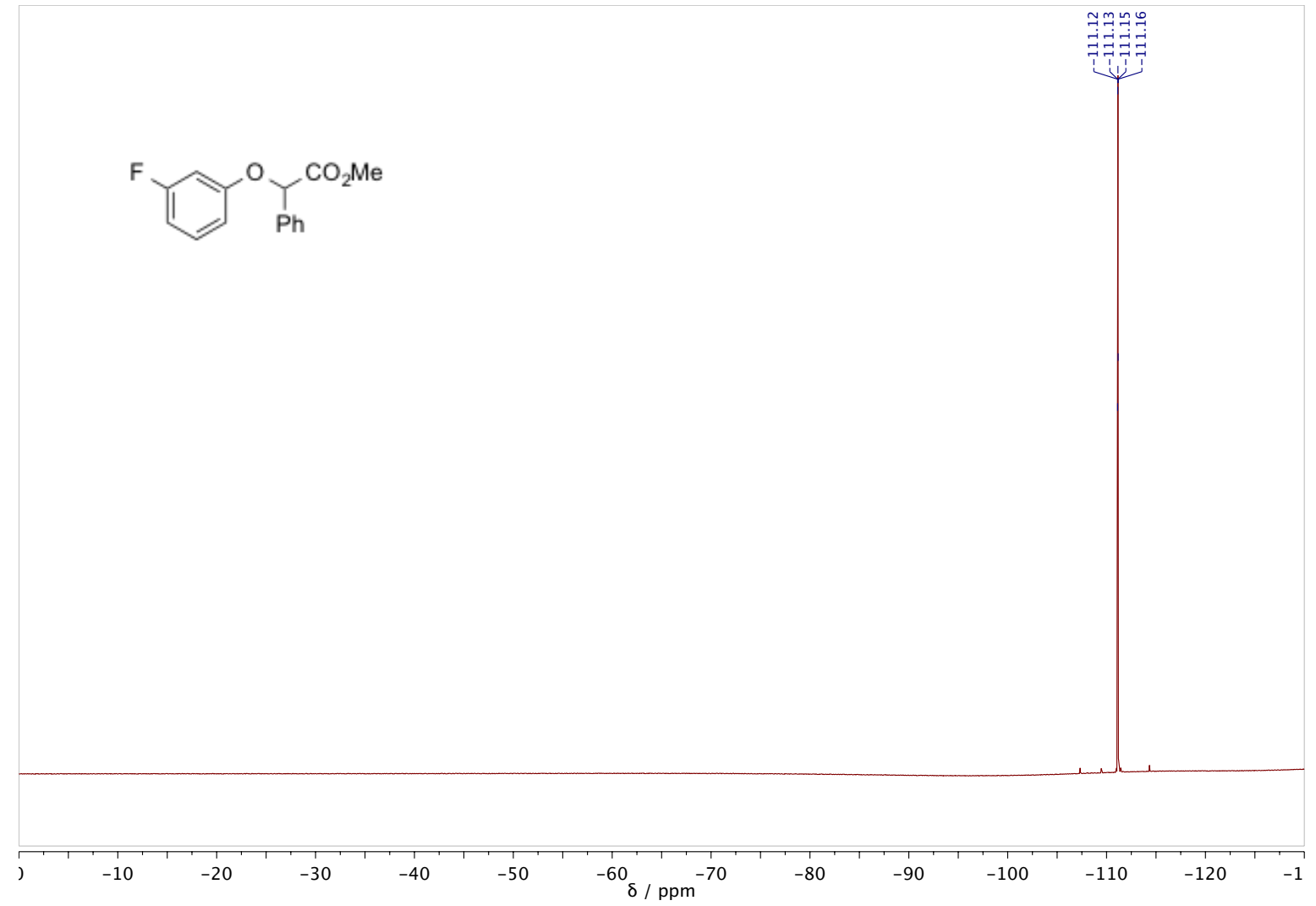


Methyl 2-phenyl-2-(3-(trifluoromethyl)phenoxy)acetate (15j)

${ }^{1}$ H NMR (600 MHz, Chloroform- $\left.d\right)$<smiles>CC(=O)C(Oc1cccc(C(F)(F)F)c1)c1ccccc1</smiles>

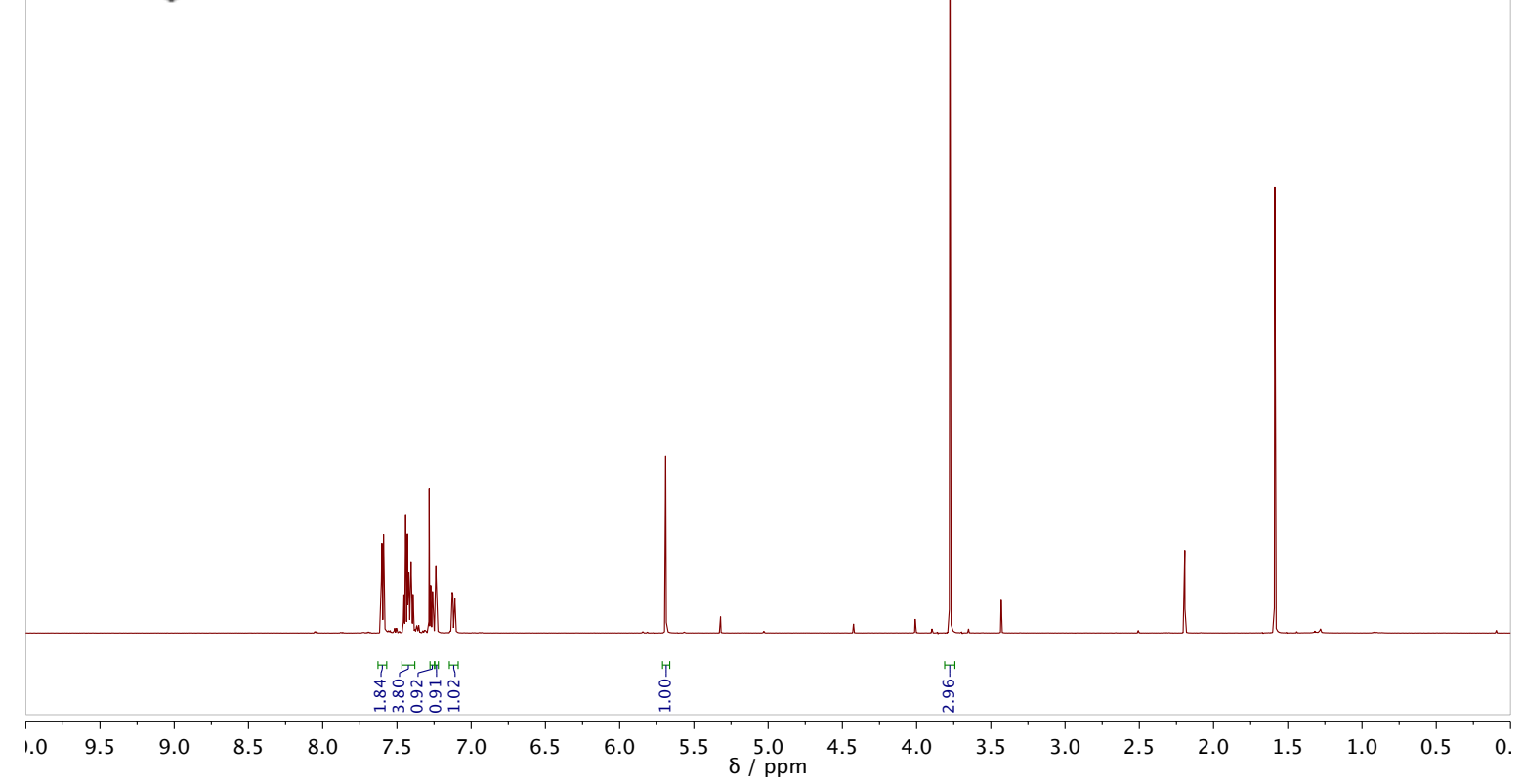

${ }^{13}$ C NMR (151 MHz, Chloroform- $d$ )

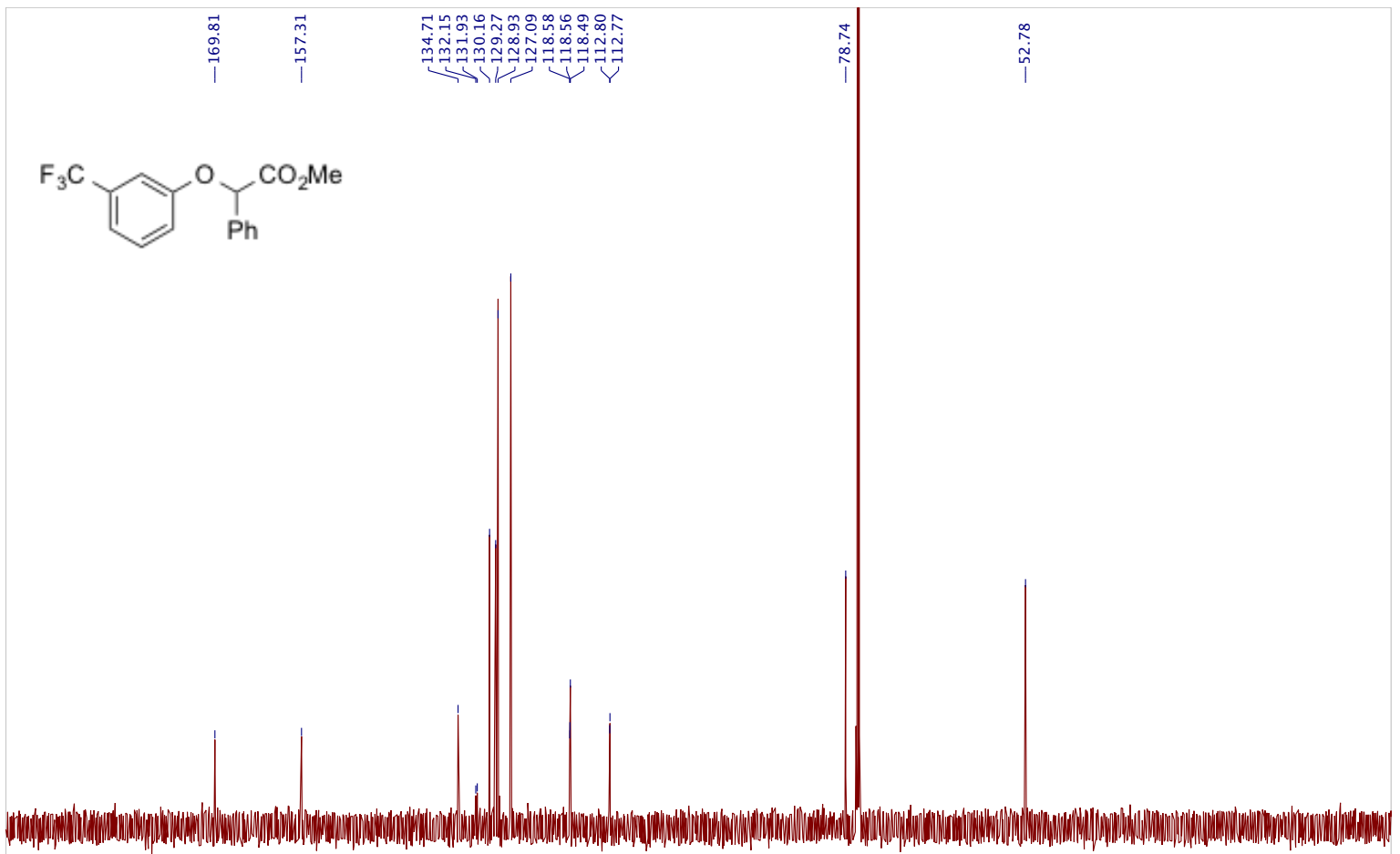

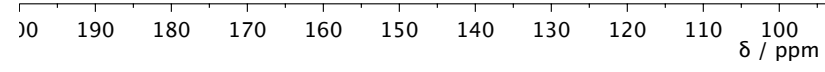


${ }^{19}$ F NMR (565 MHz, Chloroform- $d$ )

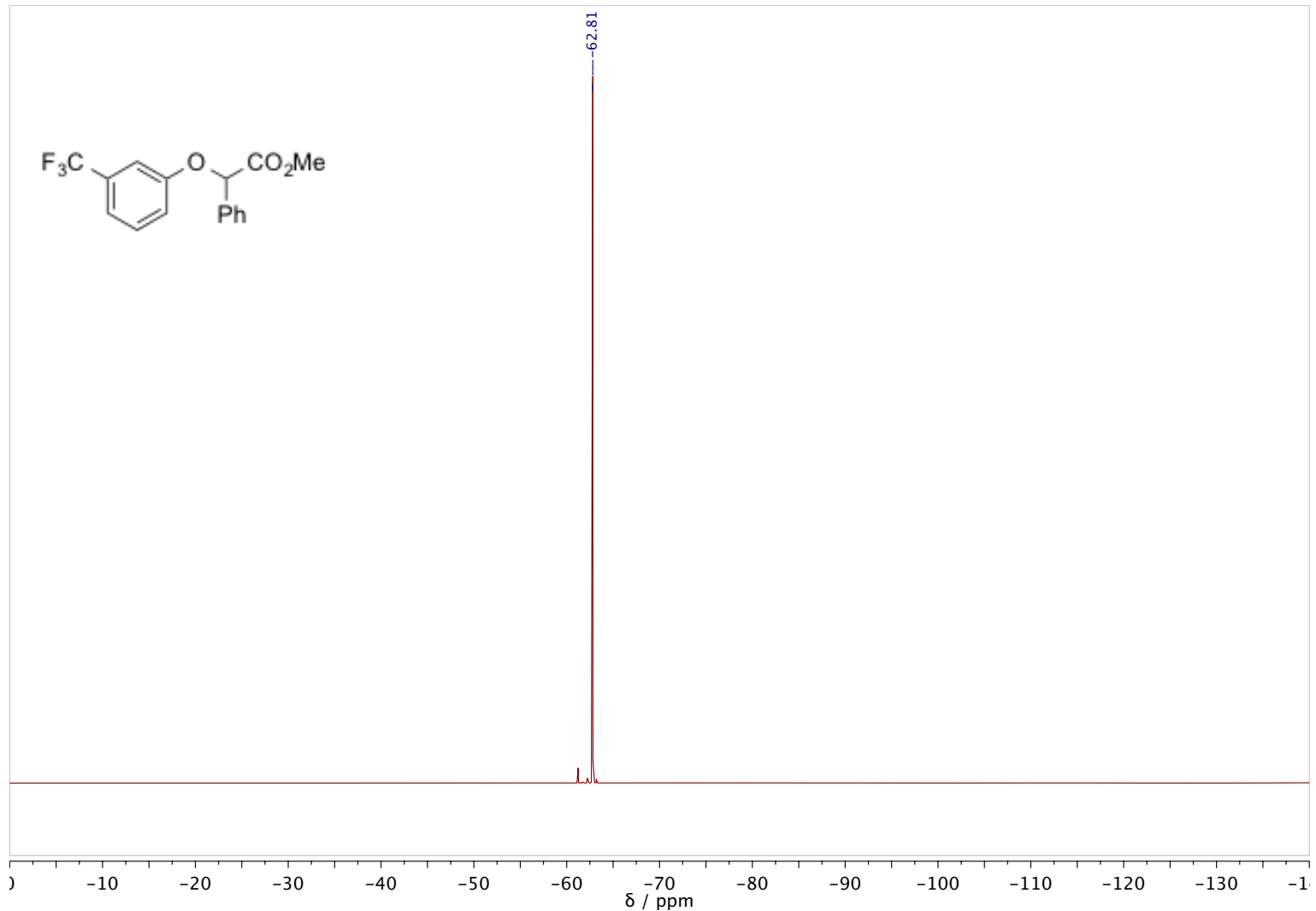


Methyl 2-(2-cyanophenoxy)-2-phenylacetate (15k)

${ }^{1}$ H NMR (600 MHz, Chloroform- $d$ )

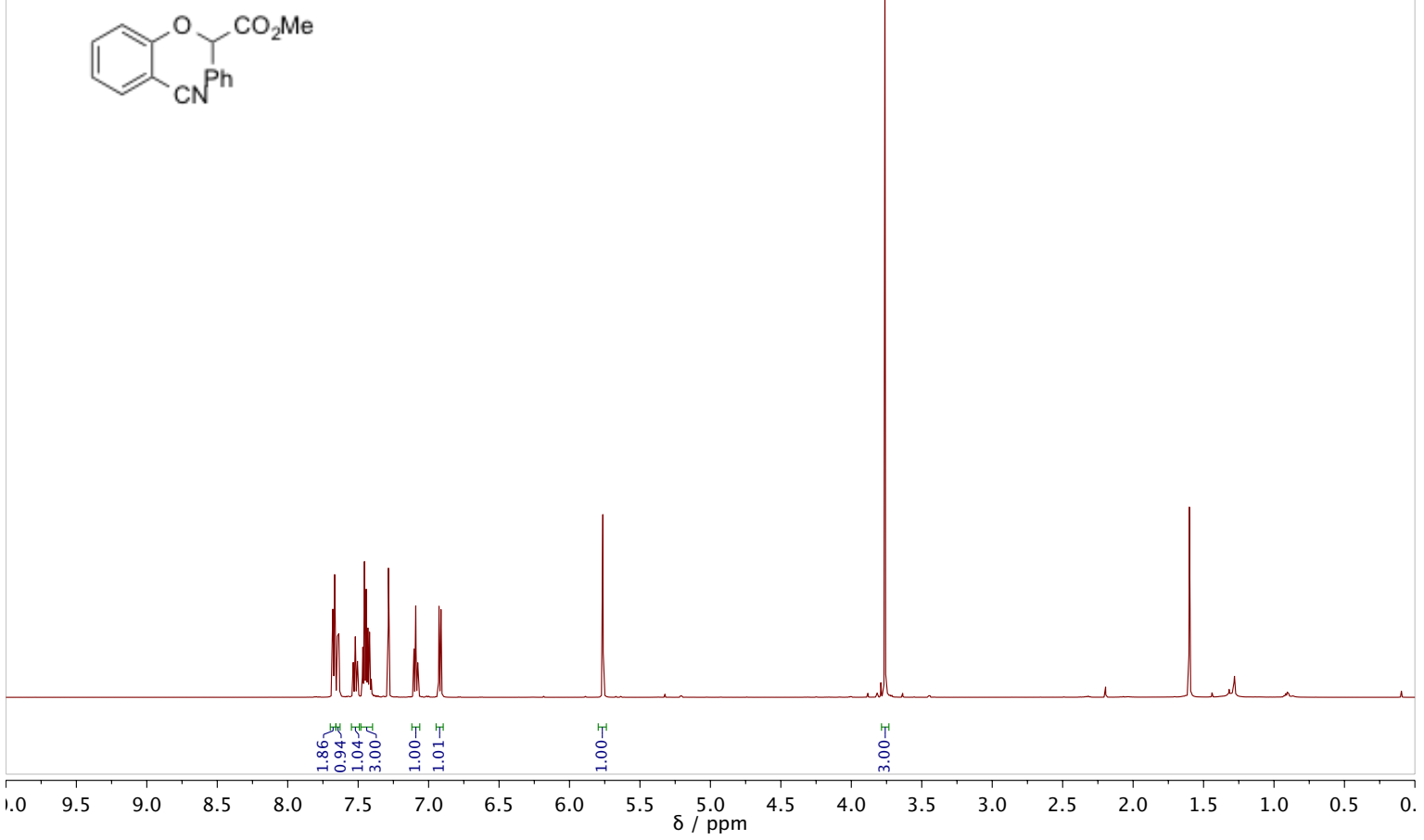

${ }^{13}$ C NMR (151 MHz, Chloroform- $d$ )

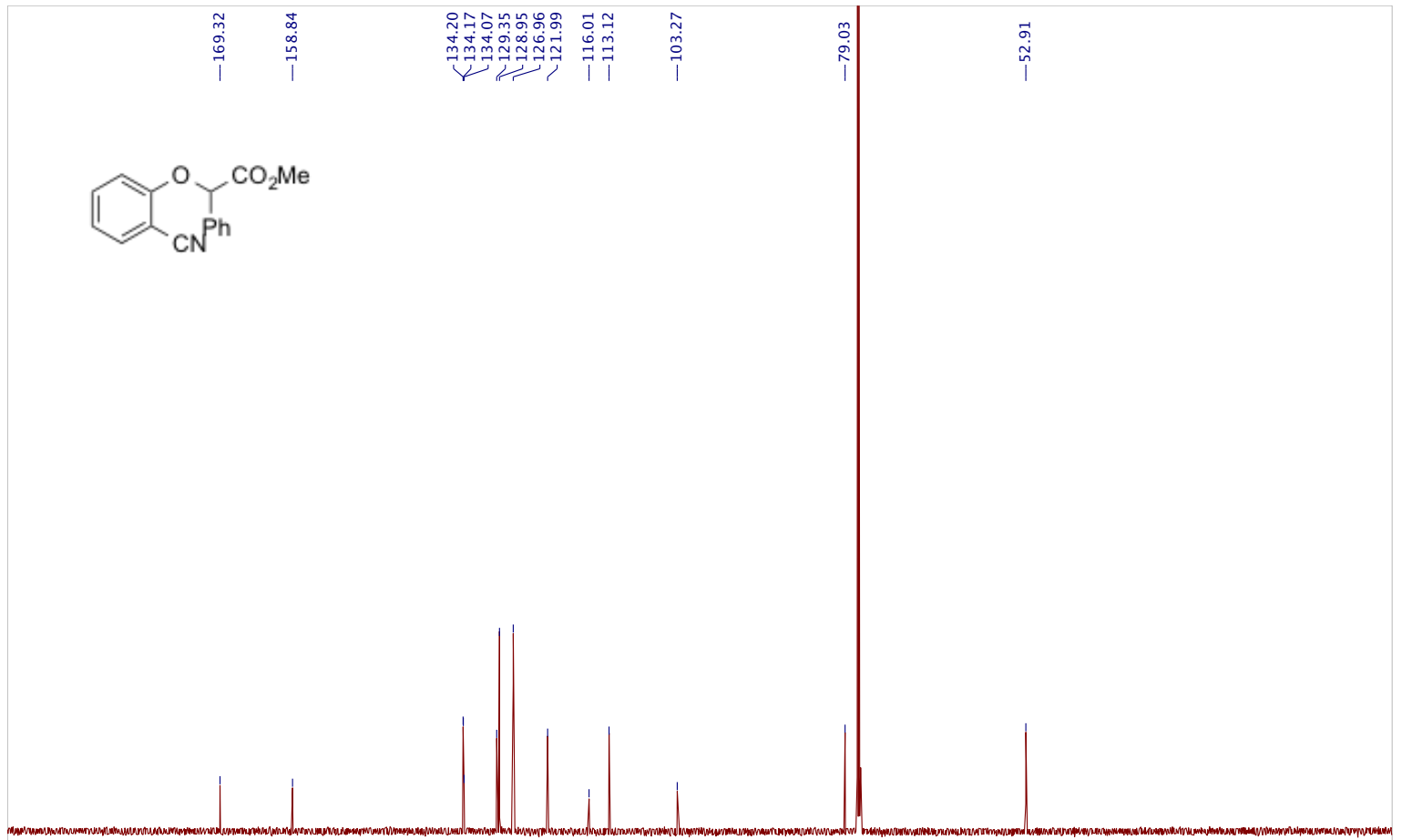

)

$\begin{array}{llllllllll}190 & 180 & 170 & 160 & 150 & 140 & 130 & 120 & 110 & \begin{array}{l}100 \\ \delta / \mathrm{ppm}\end{array}\end{array}$ 
Methyl 2-(2-nitrophenoxy)-2-phenylacetate (15l)

${ }^{1}$ H NMR (400 MHz, Chloroform-d)

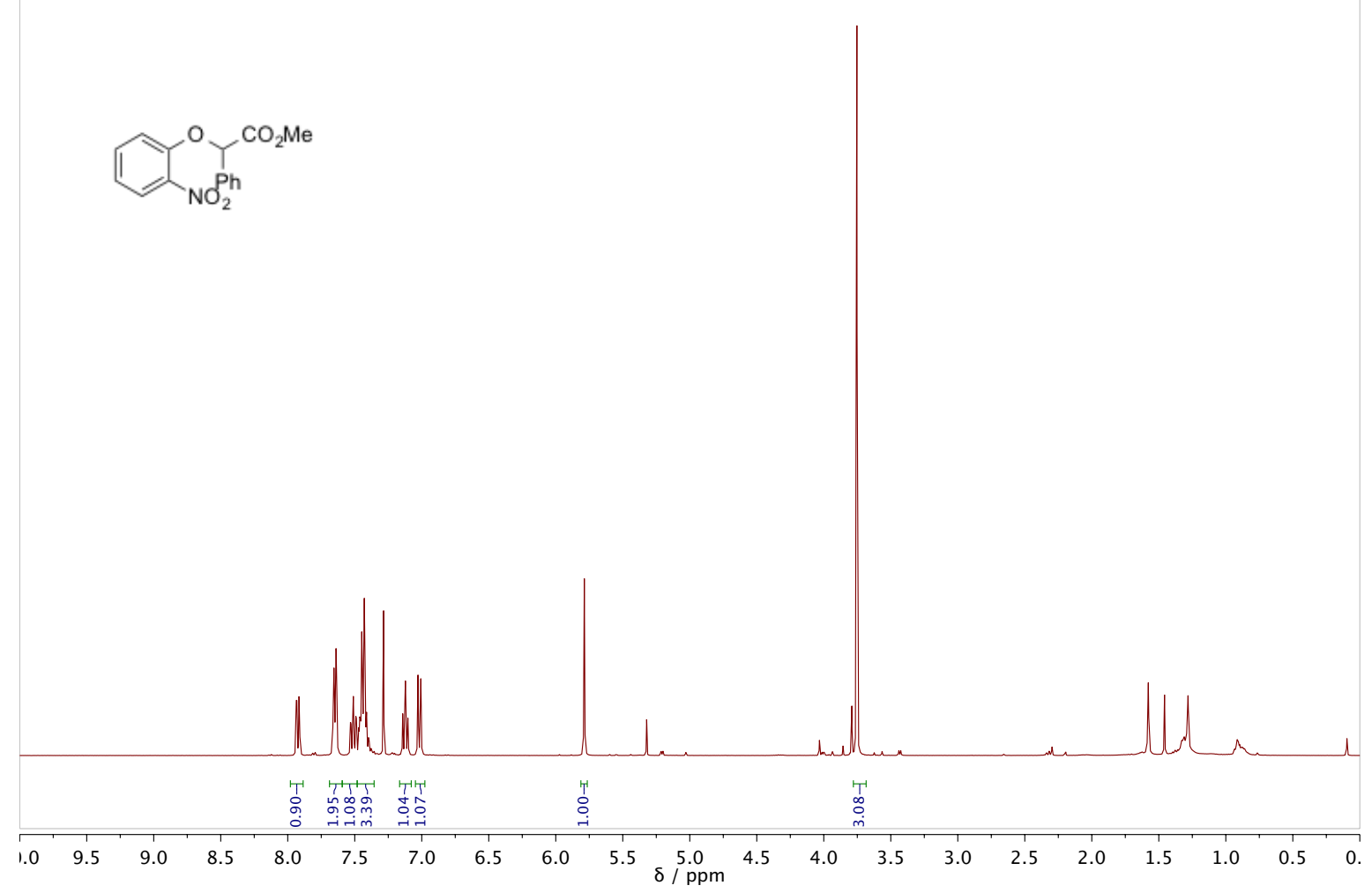

${ }^{13}$ C NMR (101MHz, Chloroform- $d$ )

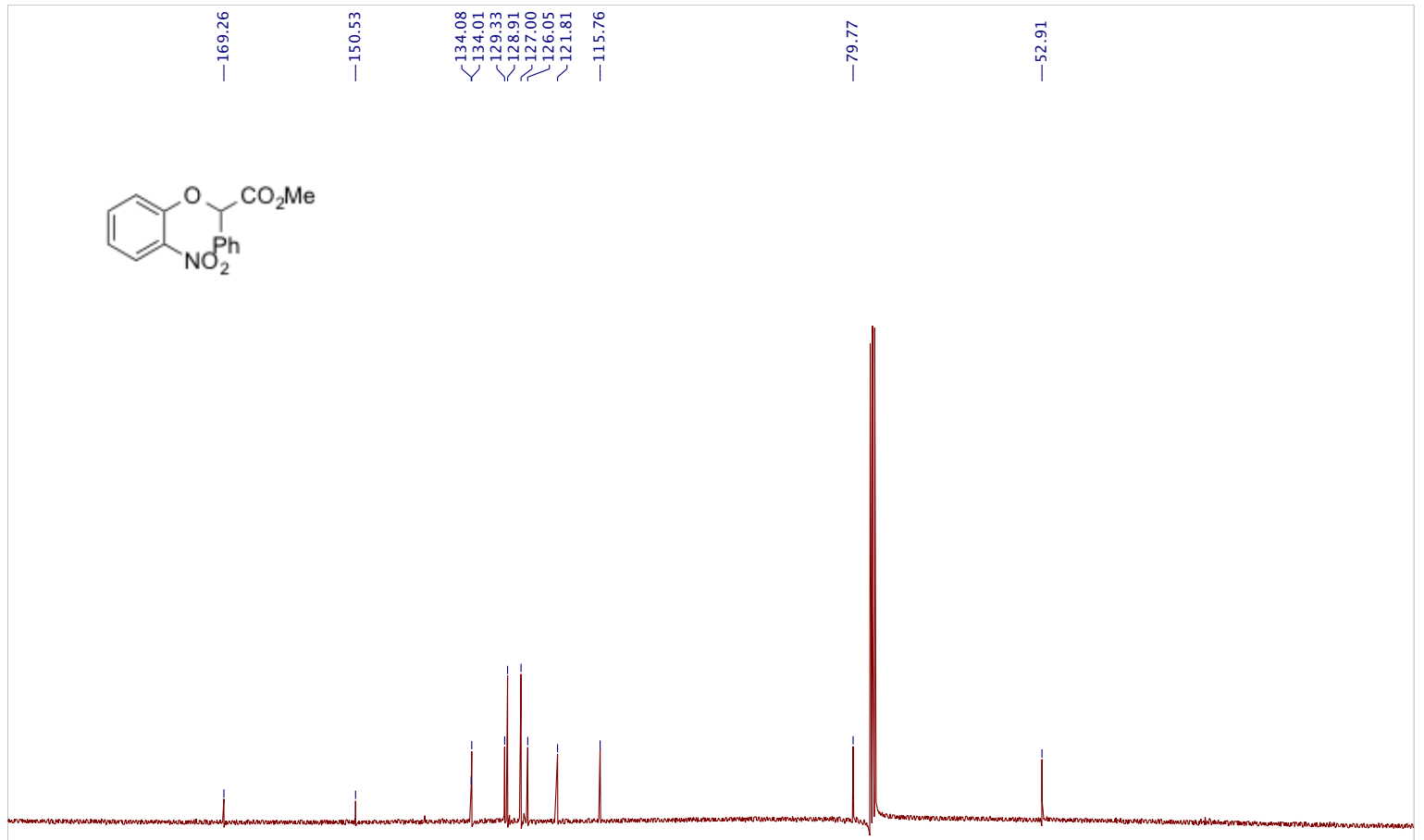

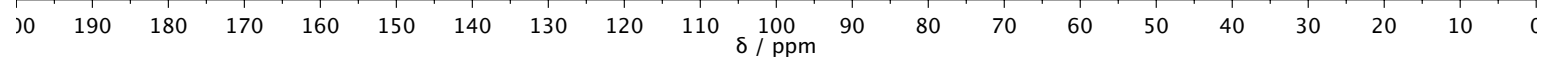


Methyl 2-(2-chlorophenoxy)-2-phenylacetate (15m)

${ }^{1}$ H NMR (600 MHz, Chloroform- $\left.d\right)$

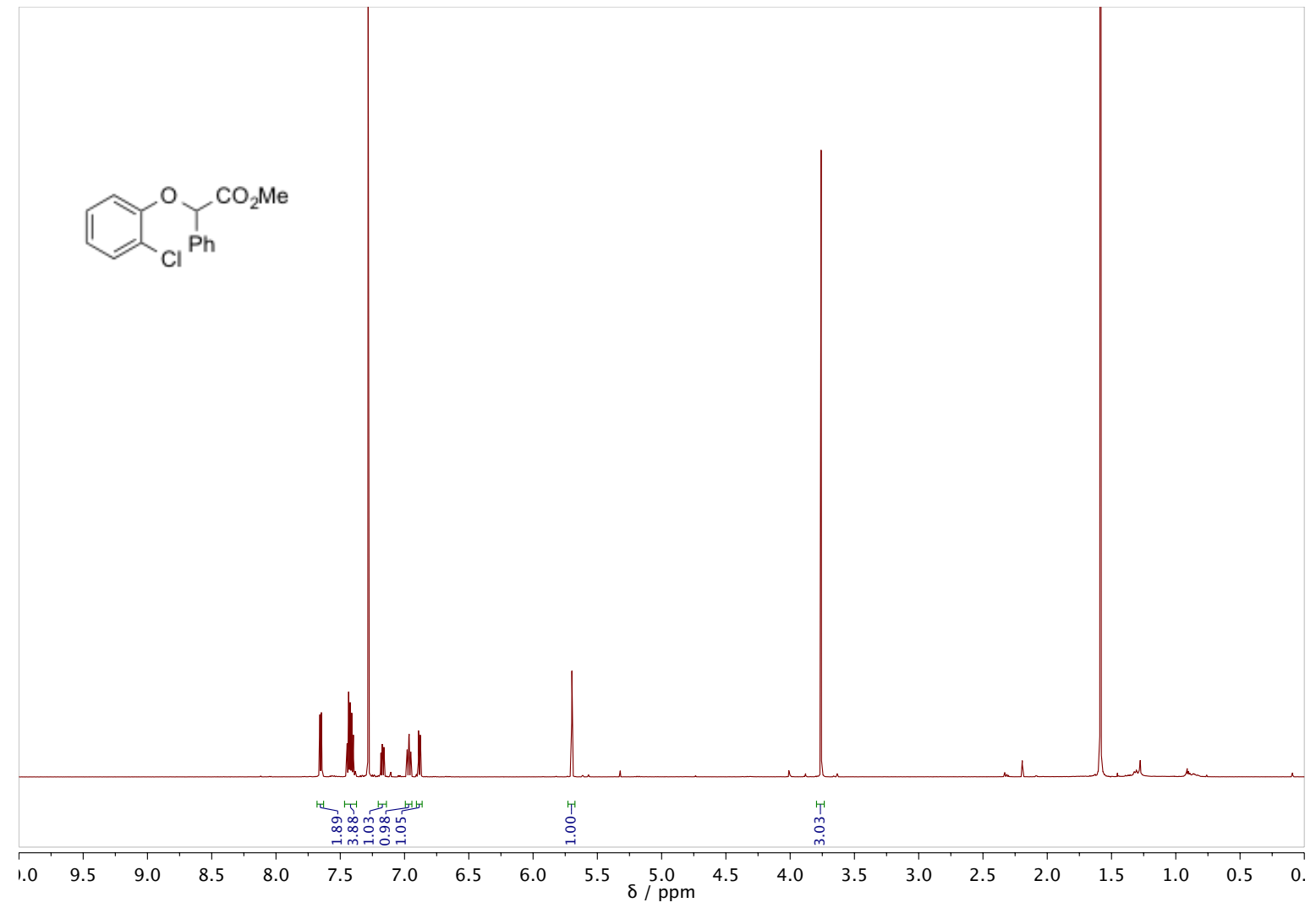

${ }^{13}$ C NMR (151 MHz, Chloroform- $d$ )

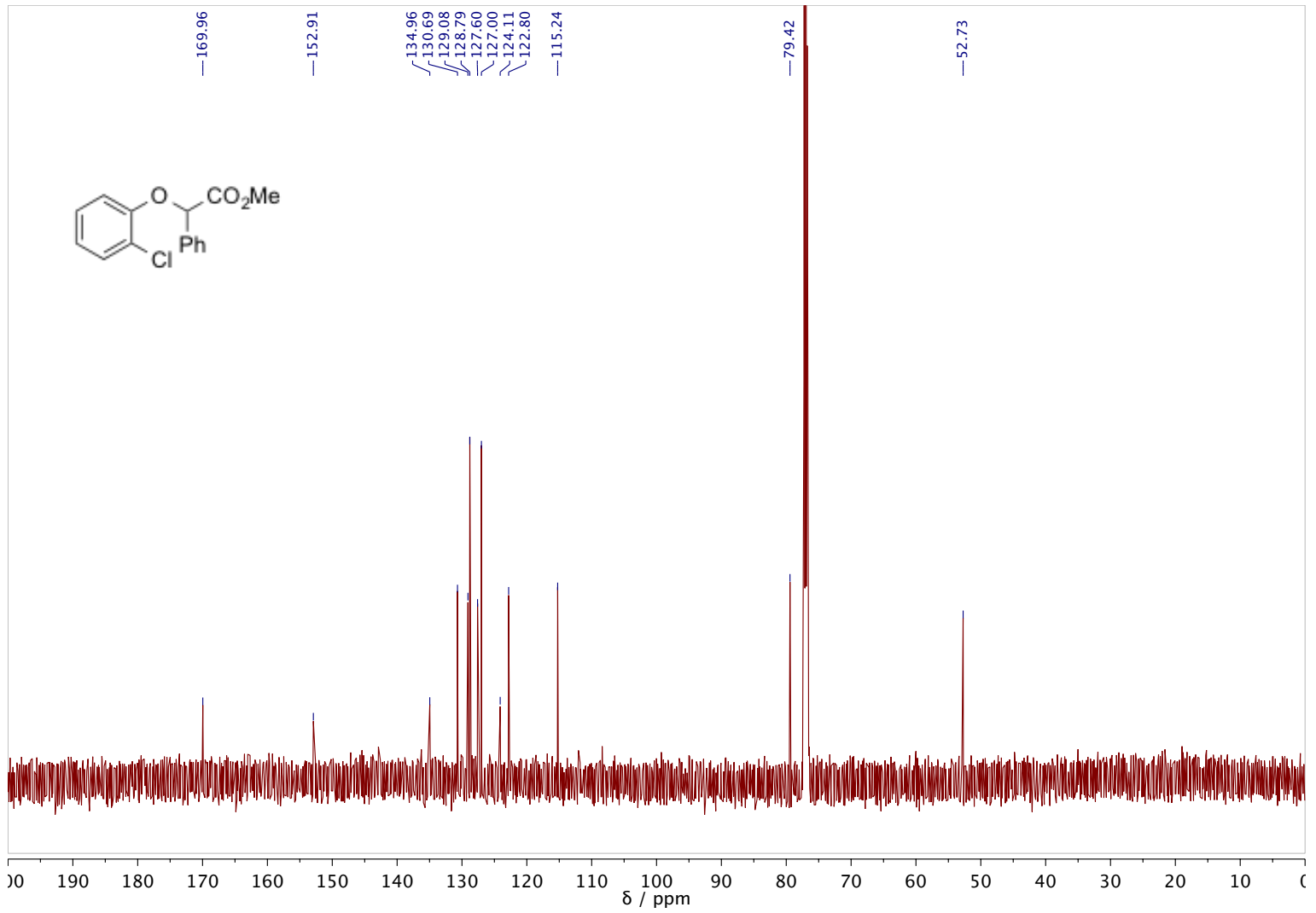


Methyl 2-(naphthalen-1-yloxy)-2-phenylacetate (15n)

${ }^{1}$ H NMR (600 MHz, Chloroform- $\left.d\right)$<smiles>CC(=O)C(Oc1cccc2ccccc12)c1ccccc1</smiles>

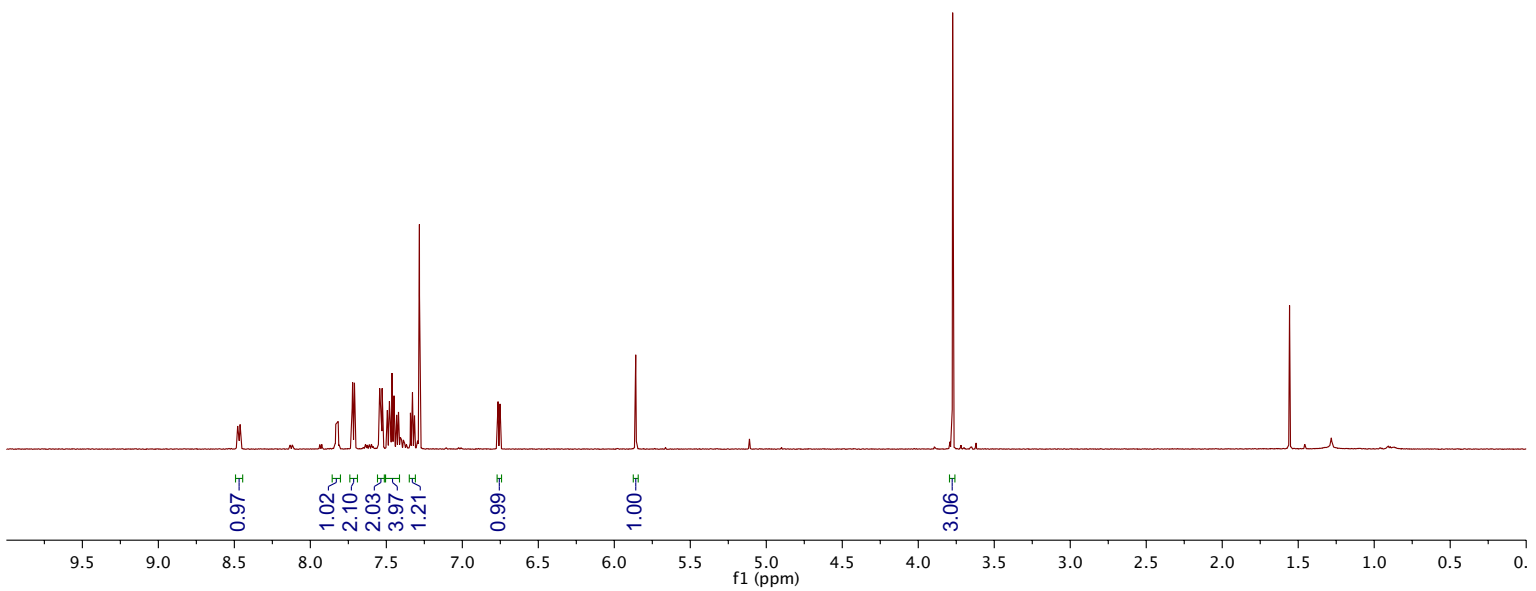

${ }^{13}$ C NMR (151 MHz, Chloroform- $d$ )

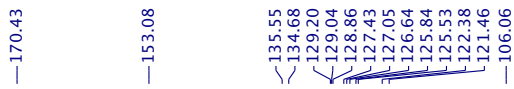

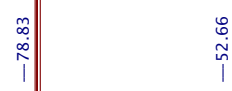

$\mathrm{NO}_{\mathrm{Ph}}^{\mathrm{O}} \mathrm{CO}_{2} \mathrm{Me}$

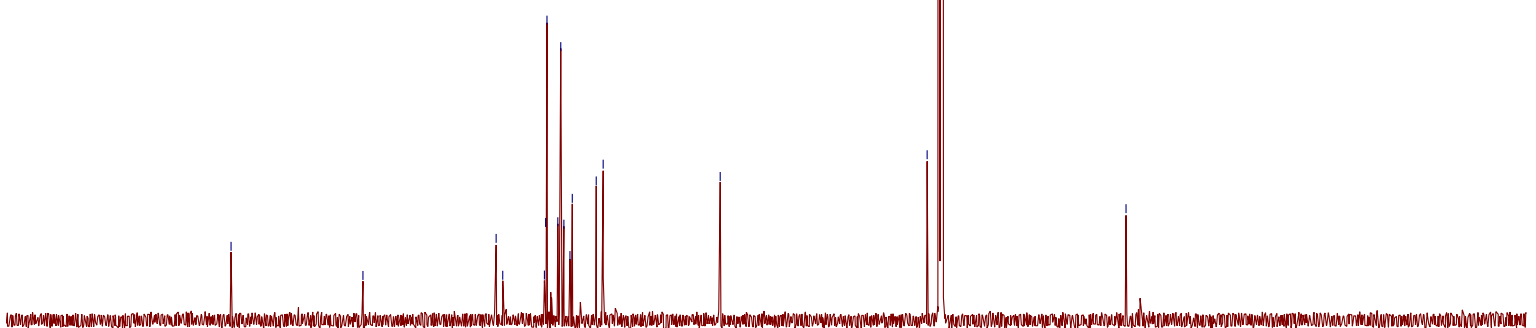

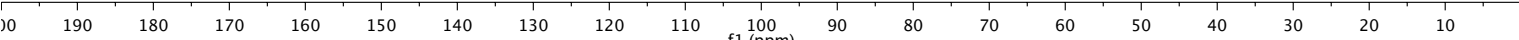


Methyl 2-phenyl-2-(pyridin-2-yloxy)acetate (150)

${ }^{1}$ H NMR (400 MHz, Chloroform-d)

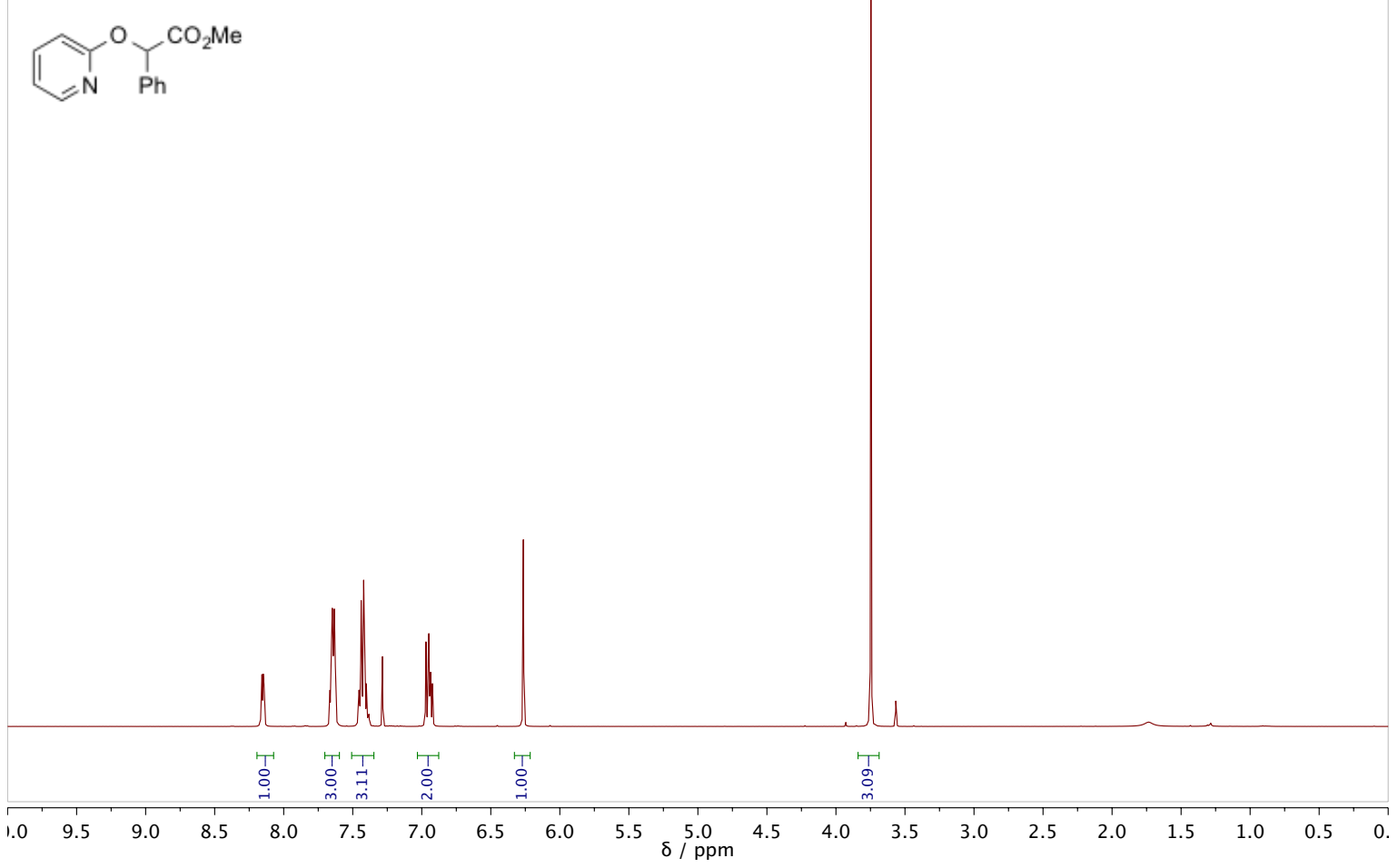

${ }^{13}$ C NMR (101MHz, Chloroform- $d$ )

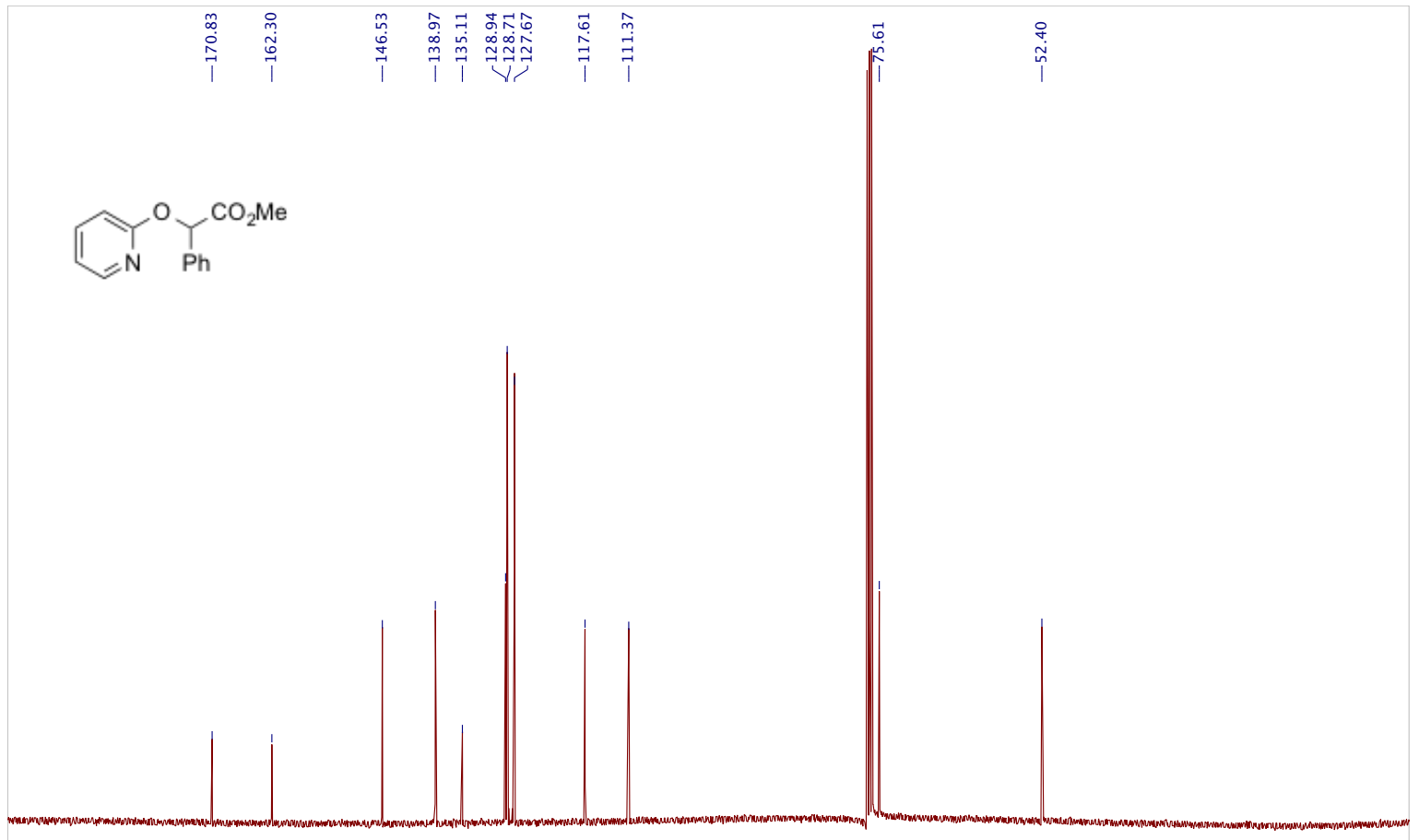

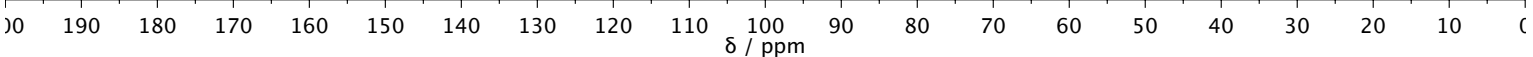


Methyl 2-phenyl-2-(quinolin-2-yloxy)acetate (15p)

${ }^{1}$ H NMR (600 MHz, Chloroform- $\left.d\right)$
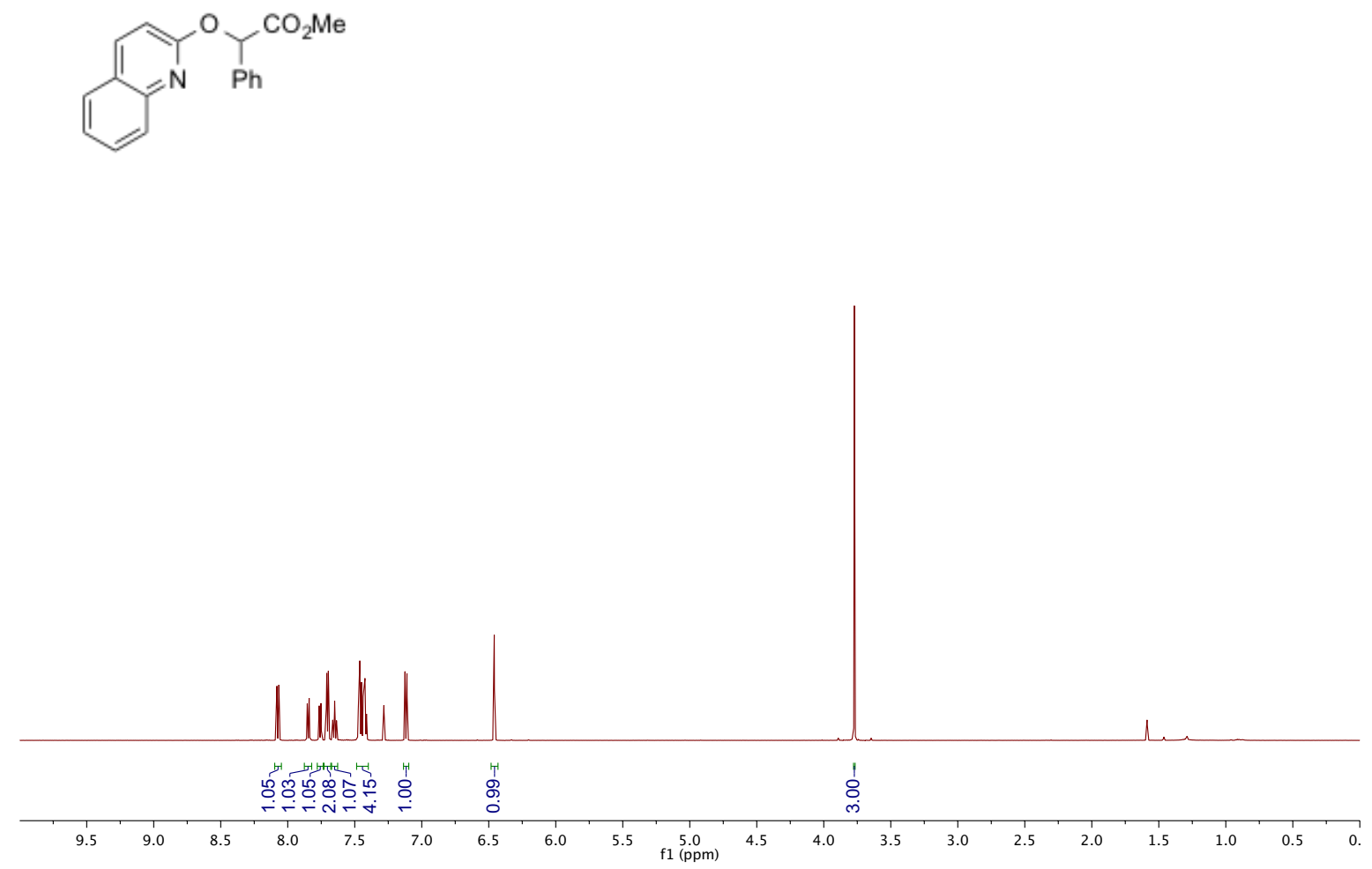

${ }^{13}$ C NMR (151 MHz, Chloroform- $d$ )
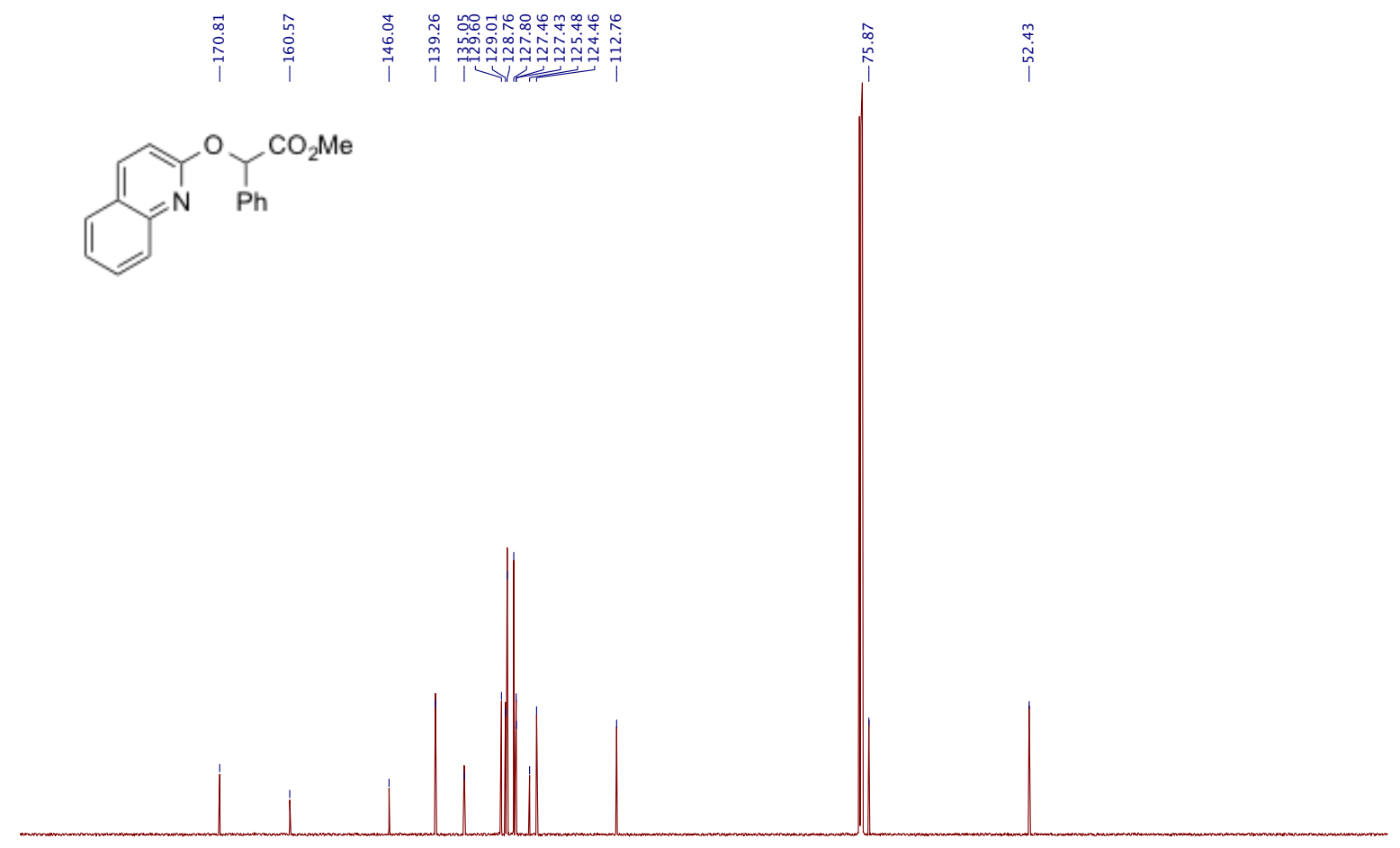

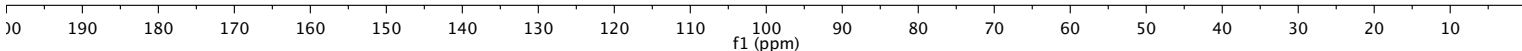

